Spätantiker Staat und religiöser Konflikt 


\section{Millennium-Studien}

zu Kultur und Geschichte des ersten Jahrtausends n. Chr.

\section{Millennium Studies}

in the culture and history of the first millennium C.E.

Herausgegeben von / Edited by

Wolfram Brandes, Alexander Demandt, Helmut Krasser,

Hartmut Leppin, Peter von Möllendorff

Band 34 


\title{
Spätantiker Staat und religiöser Konflikt
}

\author{
Imperiale und lokale Verwaltung \\ und die Gewalt gegen Heiligtümer
}

Herausgegeben von

Johannes Hahn

De Gruyter 
Diese Publikation wurde im Rahmen des Fördervorhabens 16TOA021 - Reihentransformation für die Altertumswissenschaften („Millennium-Studien“) mit Mitteln des Bundesministeriums für Bildung und Forschung im Open Access bereitgestellt. Das Fördervorhaben wird in Kooperation mit dem DFG-geförderten Fachinformationsdienst Altertumswissenschaften - Propylaeum an der Bayerischen Staatsbibliothek durchgeführt.

\section{(cC) BY-NC-ND}

Dieses Werk ist lizenziert unter der Creative Commons Attribution-NonCommercial-NoDerivatives 4.0 International Lizenz. Weitere Informationen finden Sie unter http://creativecommons.org/licenses/by-nc-nd/4.0/.

Die Bedingungen der Creative-Commons-Lizenz gelten nur für Originalmaterial. Die Wiederverwenung von Material aus anderen Quellen (gekennzeichnet mit Quellenangabe) wie z.B. Schaubilder, Abbildungen, Fotos und Textauszüge erfordert ggf. weitere Nutzungsgenehmigungen durch den jeweiligen Rechteinhaber.

ISBN 978-3-11-024087-0

e-ISBN 978-3-11-024088-7

ISSN 1862-1139

Library of Congress Cataloging-in-Publication Data

A CIP catalogue record for this book is available from the Library of Congress.

Bibliografische Information der Deutschen Nationalbibliothek

Die Deutsche Nationalbibliothek verzeichnet diese Publikation in der Deutschen Nationalbibliografie; detaillierte bibliografische Daten sind im Internet über http://dnb.d-nb.de abrufbar.

(C) 2011 Walter de Gruyter GmbH \& Co. KG, Berlin/New York

Druck: Hubert \& Co. GmbH \& Co. KG, Göttingen

$\infty$ Gedruckt auf säurefreiem Papier

Printed in Germany

www.degruyter.com 


\section{Inhaltsverzeichnis}

JOHANNES HAHN

Spätantiker Staat und religiöser Konflikt - Einleitende Bemerkungen

MARTIN WALLRAFF

Die antipaganen Maßnahmen Konstantins in der Darstellung

des Euseb von Kaisareia

FRANK R. TROMBLEY

The Imperial Cult in Late Roman Religion (ca. A.D. 244-395):

Observations on the Epigraphy

GIORGIO BONAMENTE

Einziehung und Nutzung von Tempelgut durch Staat und Stadt

in der Spätantike

ECKHARD MEYER-ZWIFFELHOFFER

Mala desidia iudicum? Zur Rolle der Provinzstatthalter bei der

Unterdrückung paganer Kulte (von Constantin bis Theodosius II.)

ULRICH GOTTER

Zwischen Christentum und Staatsraison. Römisches Imperium

und religiöse Gewalt

HANS-ULRICH WIEMER

Für die Tempel? Die Gewalt gegen heidnische Heiligtümer

aus der Sicht städtischer Eliten des spätrömischen Ostens

BRYAN WARD-PERKINS

The End of the Temples: An Archaeological Problem

JOHANNES HAHN

Gesetze als Waffe? Die kaiserliche Religionspolitik und

die Zerstörung der Tempel 



\title{
Spätantiker Staat und religiöser Konflikt - Einleitende Bemerkungen
}

\author{
JOHANNES HAHN
}

Gewalt gegen Heiligtümer, Zerstörungen von Tempeln und Umwandlungen von Kultstätten in christliche Kirchen bedeuten zentrale Symbole für den Wandel der religiösen Verhältnisse, des öffentlichen Lebens und des sozio-politischen Systems der Spätantike. Tatsächlich stellten Übergriffe gegen pagane Kultorte - aber auch gegen Kirchen und Versammlungsorte christlicher Gruppen oder Synagogen - ein markantes Phänomen des religiösen, sozialen, politischen und kulturellen Umbruchs im Imperium Romanum in der Zeit von Konstantin bis Justinian dar. Die sich hier artikulierende neue Erscheinungsweise gewaltsamer öffentlicher Auseinandersetzung, das religiös motivierte Vorgehen gegen Orte, Objekte oder Personen, repräsentierte, in den Städten wie auf dem Land, vor allem eine neue Form des Konflikts: Es brachen hier bislang kaum bekannte Spannungslinien im Gefüge von Lokalgesellschaften auf, und es traten Gruppen als Akteure in Erscheinung, die bislang nicht militant hervorgetreten waren, ja in der Öffentlichkeit zuvor teils kaum als solche wahrgenommen worden waren. Nicht zuletzt sah sich der römische Staat nunmehr mit vormals gänzlich unbekannten Herausforderungen konfrontiert.

In der Spätantike standen sich mit Juden, Christen und Anhängern paganer Kulte im öffentlichen Raum konkurrierende Gruppen gegenüber, für deren Selbstdefinition die Zugehörigkeit zu einer Kult- bzw. einer Religionsgemeinschaft wesentlich oder sogar ausschlaggebend war. Zwischen ihnen kam es im Rahmen lokaler Konstellationen immer wieder zu Auseinandersetzungen, die im äußersten Fall die Existenz des jeweils anderen zur Disposition stellten. Die radikalste Form des Umgangs mit dem religiösen alter war das Vorgehen gegen dessen Kultorte. So wurden die Heiligtümer und die damit verbundenen Riten regelmäßig zu Kristallisationspunkten militanter Auseinandersetzungen. Zugleich entschied sich hier die Verfügungsmacht über weitreichende ökonomische und soziale Ressourcen und stand die Balance gesellschaftlicher und politischer Verhältnisse zur Disposition.

Diese Ausübung von Gewalt forderte notwendigerweise alle staatlichen Institutionen heraus. Das galt ebenso für den Herrscher und die imperiale Zentrale wie für die Reichsadministration vom Prätorianerpräfekten über die Provinzstatthalter und Truppenkommandeure bis zu den lokalen Amtsträgern und Stadtverwaltungen. Die religiöse Gewalt der Spätantike sorgte allerdings darüber hinaus für ein 
scharfes Aufeinanderprallen von Tradition und Neuerung. Denn grundsätzlich hatte die imperiale Verwaltung an der Veränderung bewährter Ordnung und Strukturen und erst recht an eskalierenden Konflikten im Reich kein Interesse. Traditionell suchte sie gewaltsame Konflikte innerhalb des Reiches vielmehr nach Kräften einzudämmen, ja sah in der Bewahrung der öffentlichen Ordnung und Gewährleistung der pax Romana bzw. pax Augusta immer den eigentlichen Primat ihres Handelns. Doch erlangte der neue Glaube, mit dem sich Gewalt nunmehr als religiöse Agenda begründen ließ, rapide zunehmende Bedeutung für alle Konfliktparteien - aber nicht allein für diese: auch für den Kaiser (und sein Selbstverständnis) und gleichermaßen die städtischen Eliten. So brachte der Weg zum christlichen Imperium eine Vielfalt einschneidender Veränderungen mit sich: Der Angriff auf Heiligtümer aber verwandelte das Gesicht der Städte und veränderte das Machtgefüge auf lokaler Ebene. Im Schatten religiöser Konflikte gelang es den Bischöfen und religiösen Charismatikern (etwa Mönchsführern und Asketen), sich neben der Administration als lokale Autoritäten zu etablieren.

Nachdem unlängst eine Reihe verwandter Aspekte des Phänomens Tempelzerstörung an anderem Ort hatte erörtert werden können, ${ }^{1}$ steht im Fokus des vorliegenden Bandes die Perspektive der staatlichen Macht auf Übergriffe gegen Kultorte. Untersucht wird vor diesem Hintergrund zum einen das Verhältnis der einzelnen Ebenen der kaiserlichen Verwaltung zueinander, zum anderen das Verhältnis der staatlichen Institutionen $\mathrm{zu}$ den sich neu strukturierenden regionalen und lokalen Öffentlichkeiten und hier besonders den Schlüsselfiguren des religiösen Lebens. In den Blick zu nehmen sind dabei allerdings ebenso die Gruppen und Personen, von denen religiöse Gewalt ausging, und es ist zu fragen, an welchen Orten und in welchen Kontexten es zu Spannungen durch militante Akte kam.

Vor allem aber sind die Handlungsziele und -möglichkeiten des Kaisers und seiner Verwaltung angesichts spontaner und organisierter religiöser Gewalt zu analysieren. Ihre Interventionen, Verordnungen und Gesetze - wie überhaupt die zunehmend anti-pagan ausgerichtete sowie auf die Etablierung einer gesellschaftlich einflussreichen Reichskirche zielende Politik des Kaiserhofes, welche die Christianisierung des Reiches beförderte - bestimmten entscheidende Rahmenbedingungen für die Austragung religiöser Konflikte. Die daraus resultierenden Handlungsspielräume auf regionaler und lokaler Ebene wiederum eröffneten weitere Aktionsmöglichkeiten für ziel- und machtbewusste Persönlichkeiten und Interessengruppen. Das Zusammenwirken von Instanzen der Reichsverwaltung mit Vertretern der Kirche bei religiösen Auseinandersetzungen erweist sich hierbei heuristisch als besonders aufschlussreich.

Das skizzierte Forschungsinteresse verweist zugleich auf einen weiter gesteckten Problemrahmen, der über Fragen wie die Modalitäten öffentlicher Gewaltkontrolle, die Wirkungen wechselnder religionspolitischer Positionierungen einzelner

1 J. HAHN - S. EMmEL - U. GOTTER (Hrsg.), From Temple to Church: Destruction and Renewal of Local Cultic Topography in Late Antiquity (Religions in the Graeco-Roman World 163), Leiden 2008. 
Herrscher oder die Einschätzung von Faktoren und Dynamik des Christianisierungsprozesses in der Spätantike deutlich hinausreicht. Ausgehend von der wirkungsmächtigen These Fergus Millars vom ,government by response“ hat eine jahrzehntelange Debatte über Staatlichkeit und politisches Agieren in der römischen Kaiserzeit die relevanten Voraussetzungen und Rahmenbedingungen ausgeleuchtet und hierüber die Handlungsmuster und Handlungsspielräume politischer Akteure unter den Verhältnissen der politischen Strukturen des Imperium Romanum zu bestimmen gesucht. Vor allem der dominierend reaktive Charakter des kaiserlichen Regierungshandelns im Prinzipat wurde, bei allen notwendigen Modifikationen, so herausgestellt. ${ }^{2}$

Diese grundlegende Diskussion ist erst unlängst auch auf das spätantike Imperium Romanum ausgedehnt worden, ${ }^{3}$ welches, traditionell als autokratischer Obrigkeitsstaat mit stark zentralistischen Zügen wahrgenommen, mittels seines deutlich ausgeweiteten und hierarchisch strukturierten Verwaltungsapparates, sowie mit seinen großen systematisierenden Rechtskodifikationen, prima facie ein weit stärkeres aktives Regierungshandeln und einen markant gestiegenen Regelungsanspruch in immer neuen Alltagsfeldern signalisiert. Das von der älteren Forschung gewonnene Bild eines spätantiken ,Zwangsstaates' wurde nicht zuletzt auch an der in den Rechtskodizes umfänglich berücksichtigten Religionsgesetzgebung festgemacht. Doch eine eingehende Auseinandersetzung mit den religiösen Konflikten und den Ausbrüchen religiös motivierter Gewalt in der Spätantike - der nunmehr weitaus häufigsten und zudem am dichtesten dokumentierten Kategorie öffentlichen Aufruhrs ${ }^{4}$-, wie sie in den Beiträgen dieses Bandes unter weitgefächerter Perspektive geführt wird, gelangt hier zu sehr differenzierten Schlussfolgerungen: Die Vorstellung eines dirigistischen, auf strategischen, am Kaiserhof im sacrum consistorium entwickelten Planungen beruhenden Herrschaftssystems, das seinen dezidierten Gestaltungswillen in allgemeinen Normsetzungen, in leges generales, propagiert und zugleich mit spezifischen Ausführungsbestimmungen und mithilfe eines effizienten Herrschaftsapparates durchsetzt und auf konkrete Einzelfälle anwendet, erweist sich als bestenfalls unvollständig, wenn nicht als irreführend. Bereits die nähere Prüfung der Eigenart und Genese der Kaiserkonstitutionen in den Kodizes verweist auf ihren höchst unterschiedlichen Hintergrund,

2 H.-U. WIEMER bietet in der Einleitung zu dem von ihm herausgegebenen Sammelband, Staatlichkeit und politisches Handeln in der römischen Kaiserzeit (Millennium-Studien 10), Berlin 2006, einen instruktiven forschungsgeschichtlichen Überblick über die Diskussion, die von von F. MILLAR mit seinem Buch The Emperor and the Roman World (31 BC - AD 337), London 1977, angestoßen wurde.

3 Siehe nur einerseits F. Millar, A Greek Roman Empire. Power and Belief under Theodosius II (408-450) (Sather Classical Lectures 64), Berkeley 2006, andererseits S. SCHMIDT-HOFNER, Reagieren und Gestalten. Der Regierungsstil des spätrömischen Kaisers am Beispiel der Gesetzgebung Valentinians I. (Vestigia 58), München 2008.

4 Die von A.H.M. Jones (The Later Roman Empire 284-602. A Social, Economic and Administrative Survey, I, London 1964, 694) in seiner einflussreichen Typologie noch unterschiedenen Hungeraufstände und Zirkusrevolten sind zudem weniger komplex und beschränken sich auf Städte. 
Charakter und auch Geltungsanspruch: ein Sachverhalt, den die Arbeit der Kompilatoren nur auf den ersten Blick verdecken kann, der aber für die historische Interpretation der Gesetze - bzw. der von ihnen erhalten gebliebenen Ausschnitte und Exzerpte - umso mehr quellenkritische Distanz und Methode fordert.

Auch der politische Gestaltungswille und in vielen Fällen selbst seine spätere exekutive Umsetzung beruhten in der Spätantike immer wieder auf Initiativen von außen, auf Petitionen und suggestiones: von staatlichen Amtsträgern, aber auch Stadträten und Privatpersonen, und nicht zuletzt von Bischöfen. Gerade die Lobbyarbeit der katholischen Kirche - mittels zahlloser Briefe, Eingaben, persönlicher Vorsprache u.a. - und ihre schier nicht enden wollenden, für manche Ansprechpartner kaum noch erträglichen Anstrengungen, ${ }^{5}$ kaiserliche Entscheidungen und Gesetze, oft gegen innerkirchliche Gegner oder konkurrierende religiöse Gruppen, zu erwirken, ja unmittelbar staatliche Machtmittel zur Durchsetzung ihrer Ziele in Bewegung zu setzen, unterstreichen im Feld der Religionspolitik und -gesetzgebung, welche Bedeutung Kommunikation und Einflussnahme, auch jenseits der festgelegten Instanzenzüge, für das Tätigwerden und Funktionieren des spätrömischen Staates hatten: Nicht nur kaiserliche Entscheidungen, auch umfassende Gesetzgebung war das Ergebnis vielfältiger Einflussnahmen und aufwendiger Aushandlungsprozesse.

Der zeitliche Bogen der in diesem Band versammelten Beiträge erstreckt sich von der Mitte des 3. bis zur Mitte des 5. Jahrhunderts. Angesichts der herausragenden Bedeutung der Rechtsquellen - und hier im besonderen des Codex Theodosianus und seinem das 16. Buch (und zugleich das gesamte Werk) beschließenden 10. Kapitels mit dem Titel De paganis, sacrificiis et templis - liegt der historische Fokus allerdings auf der Zeitspanne von Konstantin bis Theodosius II. Martin Wallraff untersucht die ersten staatlichen Maßnahmen gegen pagane Kulte, welche wie die frühesten Zerstörungen von Tempeln mit der Person und Politik Konstantins verbunden sind. Nur vier Generationen später vermag der jüngere Theodosius in einem der letzten noch in seine große, $438 \mathrm{n}$. Chr. abgeschlossene Gesetzeskodifikation eingegangenen Gesetze dann - im Bewusstsein einer faktisch abgeschlossenen Christianisierung des Reiches - auf die Zerstörung oder Umnutzung von Tempeln und das Erlöschen paganer Kulte bereits als Phänomene der Vergangenheit zurückzublicken. ${ }^{6}$ Johannes Hahn hinterfragt die Repräsentativität, Aussage-

5 Die Lobbyarbeit der afrikanischen katholischen Kirche am Kaiserhof in Ravenna nahm im Jahr 405 solch unerträgliche Ausmaße an, dass der Hof der Kirche in Karthago über den römischen Bischof unmissverständlich mitteilen ließ, künftig von Gesandtschaften mit überflüssigen Eingaben abzusehen. Dem Papst oblag es nunmehr, jede einzelne Petition aus Africa zuvor abzuzeichnen: Recitatae litterae papae Innocentii, ut episcopi ad transmarina pergere facile non debeant: quod hoc ipsum episcoporum sententiis confirmatur. (Conc. Carth., 13 Iun. $407=$ Reg. Eccl. Carth. Excerpt. XII 106 (CCL 149: 218f., Munier)). Hierzu J.E. MERDINGER, Rome and the African Church in the Age of Augustine, New Haven 1997, 98f. Vgl. auch CTh 12,12,6.

6 CTh 16,10,25 vom 14. Nov. 435 formuliert: ... sacrificiis ceterisque antiquiorum sanctionum auctoritate prohibitis interdicimus cunctaque eorum fana templa delubra, si qua etiam nunc 
kraft und Wirksamkeit dieser in retrospektiver Perspektive von Theodosius' Juristen kanonisierten Gesetzestexte für das Ende des öffentlichen Heidentums.

Die materielle Seite dieses facettenreichen Prozesses des Niedergangs und der Zerstörung der paganen Infrastruktur im Imperium untersuchen Bryan WardPerkins, der die Möglichkeiten, vor allem aber auch die methodischen Grenzen der historischen Interpretation archäologischer Befunde in Hinblick auf das Ende der Tempel reflektiert, und Giorgio Bonamente, der den Verbleib der Tempelinventare und -liegenschaften, ihre Einziehung und Nutzung durch öffentliche Institutionen, auf der Basis der literarischen und juristischen Überlieferung prüft. Eine umfassende Bestandsaufnahme der epigraphischen Überlieferung in den östlichen Provinzen liegt der Untersuchung des Kaiserkultes zugrunde; Frank Trombley vermag dessen ungebrochene Kontinuität bis nahezu ans Ende des 4. Jahrhunderts im einzelnen nachzuzeichnen.

Recht und Gesetz und ihre Exekution - und darüber hinaus die Frage der Legitimität von Gewalt und Widerstand - stehen im Mittelpunkt der übrigen drei Beiträge. Die schlüsselhafte Bedeutung der Provinzstatthalter für die Durchsetzung der religionspolitischen Direktiven der Zentrale auf der provinzialen und lokalen Ebene analysiert Eckhard Meyer-Zwiffelhoffer - und beleuchtet so zugleich systemische Widerstände und Widersprüche im Exekutionsapparat, welche die „tausend Strafandrohungen" der kaiserlichen Religionsgesetze zu stumpfen Instrumenten werden lassen konnten. ${ }^{7}$ Ulrich Gotter zeigt am Disput zwischen Ambrosius und Theodosius über die Frage der Bestrafung der Täter von Kallinikon, wo 388 n. Chr. erstmals eine jüdische Synagoge einem christlichen Mob (unter Führung des Ortsbischofs) zum Opfer fiel, wie der Mailander Kirchenführer offensiv Gewalt auf die religiöse Agenda setzt und als legitim, ja geboten begründet. Einen Religions- und Gewaltdiskurs ganz anderen Zuschnittes - aus paganer Perspektive gegen religiöse Gewalt, aber zugleich zutiefst defensiv - untersucht Hans-Ulrich Wiemer in der Schrift Pro Templis des Libanios. Nicht ernstlicher Widerstand, sondern der Respekt vor dem geltenden Gesetz und der Appell an den Kaiser als Hüter des Rechts wird hier von einem paganen Angehörigen der städtischen Eliten als Strategie zur Bewahrung entsakralisierter Kultbauten und Traditionen propagiert.

Die Beiträge zu diesem Band erwuchsen aus einer Tagung, die 2005 in Münster stattgefunden hat und welche im Rahmen des durch die Deutsche Forschungsgemeinschaft geförderten und von Johannes Hahn und Stephen Emmel geleiteten Forschungsprojektes „Kampf um Kultstätten. Sakraler Ort und religiöser Konflikt" veranstaltet werden konnte. An der Konzeption der Tagung war auch Ulrich Gotter beteiligt. Dass die Beiträge erst jetzt und unter nur einem Herausgeber erscheinen, ist verschiedenen Irrungen und Wirrungen geschuldet. Ich bitte die Leser hierfür um Nachsicht, die Autoren aber noch einmal um Entschuldigung.

restant integra ... In CTh 16,10,22 (423 n. Chr.) heißt es: ... paganos qui supersunt, quamquam iam nullos esse credamus, promulgatarum .... Vgl. auch den folgenden Text, CTh 16,10,23.

7 Nov. Theod. 3,8 (438 n.Chr.): „... promulgatarum legum mille terrores.“ 



\title{
Die antipaganen Maßnahmen Konstantins in der Darstellung des Euseb von Kaisareia
}

\author{
MARTIN WALLRAFF
}

\begin{abstract}
Während [Konstantin] seinen Erlöser und Gott ... ohne Unterlaß ehrte, brachte er auf mannigfache Art den götzendienerischen Irrtum der Heiden ans Licht. So wurden in den einzelnen Städten die Vorhöfe ihrer Tempel ganz mit Recht bloßgelegt, da sie auf Befehl des Kaisers ihrer Türen beraubt wurden. Bei anderen wurden die Dachziegel weggenommen und so das Dach über der Decke zerstört; wieder bei anderen wurden die verehrten Erzbilder, deren sich der Trug der Alten so lange Zeit gerühmt hatte, auf allen Plätzen der Kaiserstadt offen ausgestellt. ... In anderen Provinzen kamen die Bewohner scharenweise von selber zur Erkenntnis des Heiles, und sie vernichteten in Stadt und Land das als leeres Nichts, was ihnen früher für heilig gegolten hatte: die aus mancherlei Material gefertigten Götzenbilder. Ihre Tempel und Heiligtümer, die sich stolz erhoben, zerstörten sie, ohne daß es ihnen jemand befohlen hätte, erbauten dafür von Grund auf Kirchen und tauschten diese gegen ihren früheren Wahn ein. ${ }^{1}$
\end{abstract}

Wer diese Sätze des Euseb von Kaisareia liest, könnte meinen, daß alles, was sich im Laufe des vierten und fünften Jahrhunderts tatsächlich ereignen sollte, bereits unter Konstantin umfassend geplant und weitgehend durchgeführt wurde: die systematische Unterdrückung des Heidentums, das gewaltsame Vorgehen gegen Tempel mit staatlicher Unterstützung, schließlich die Ersetzung durch christliche Kultstätten. All dies aber hat, wie wir wissen, erst viel später stattgefunden Euseb legt hier geradezu hellseherische Fähigkeiten an den Tag. Nicht zuletzt solche Beobachtungen haben in der Forschung sogar zu der These geführt, die Lebensbeschreibung Konstantins sei insgesamt viel später entstanden, also nicht

1 Eus. v.C. 3,54,1 f. und 4,39,2. Die Vita Constantini wird nach der Ausgabe von WiNKELMANN ${ }^{2} 1975$ zitiert, hier $107 \mathrm{f}$. und 135. Die deutschen Übersetzungen orientieren sich im folgenden an BIGELMAIR 1913, modifizieren den dort gegebenen Text aber des öfteren. Hilfreich ist außerdem in manchen Fällen die italienische Übersetzung von TARTAGLIA ${ }^{2} 2001$ sowie vor allem die kommentierte englische Übertragung von CAMERON - HALL 1999. Neben der Vita Constantini kommt als zweite Hauptquelle Eusebs Tricennatsrede in den laudes Constantini in Frage (HEIKEL 1902; frz. Übersetzung und Anmerkungen von MARAVAL 2001). Allerdings ist in dem einschlägigen Kapitel 8 das Verhältnis von vollmundiger Rhetorik zum sachlichfaktischen Gehalt noch ungünstiger als in der Vita. Als einziger konkreter Fall ist die Aufhebung der Tempelprostitution in Heliopolis genannt (Eus. l.C. 8,5-7, s. dazu unten Anm. 21). 
Euseb zuzuschreiben oder wenigstens später stark interpoliert worden. ${ }^{2}$ Diese These hat sich nicht halten können, aber gleichwohl hat sich die Auffassung durchgesetzt, daß man Euseb zumindest in den Konstantin-Schriften nicht über den Weg trauen darf, daß die von ihm gegebenen Informationen hoffnungslos übertrieben und daher nicht ohne weitere Prüfung zu übernehmen seien. Diese Auffassung ist gewiß berechtigt - sie sollte allerdings ohne polemischen Applomb vorgetragen werden, denn die fraglichen Schriften sind enkomiastischer Gattung und streben daher nicht nach nüchterner Objektivität. Jeder antike Leser wußte genau, was er davon halten und erwarten durfte und was nicht - übrigens nicht nichts, denn der feine Unterschied zwischen (oft schamloser) Übertreibung und regelrechter Lüge wurde durchaus gewahrt, außerdem oft mit dem Mittel der partiellen Beschreibung gearbeitet, anders gesagt: mit dem gezielten Verschweigen.

Daß der christliche Bischof von Kaisareia gerade bei den antipaganen Maßnahmen des von ihm so verehrten Kaisers gerne ein bißchen vollmundiger wurde, darf nicht erstaunen, lag doch darin aus seiner Sicht ein wesentliches Spezifikum der neuen Epoche. Wir sind also gut beraten, bei allen einschlägigen Berichten a priori einige Abstriche zu machen und außerdem eine durchaus selektive Wahrnehmung vorauszusetzen. Wenn es etwa in dem eingangs zitierten Passus heißt, daß die Bewohner mancher Städte ihre Tempel freiwillig zerstörten, so sollte dies weniger als Hinweis auf tatsächlich erfolgte Tempelzerstörungen genommen werden, sondern mehr als Information, daß dergleichen staatlich eben nicht verordnet wurde (so sehr es sich vielleicht mancher rachsüchtige Christ wünschte). Und wenn in Einzelfällen von Türen oder Dachziegeln die Rede ist, sollte wiederum daraus geschlossen werden, daß es eben systematische Eingriffe in die bauliche Integrität von Tempeln in der Regel nicht gab. Auf die angeblich zum Spott ausgestellten Götterbilder komme ich gleich noch zu sprechen.

Mit der (methodisch kontrollierten) Skepsis Euseb gegenüber ist das Problem aber nicht gelöst, nicht ganz jedenfalls. Auch wenn es in jüngerer Zeit eine gewisse Tendenz gegeben hat, auch den ,paganen' Konstantin wieder stärker zu entdecken, ${ }^{3}$ können seine antipaganen Maßnahmen jedenfalls nicht ganz auf das Konto des christlichen Berichterstatters gebucht und damit aus der historischen

2 Das war die These von GRÉGOIRE 1930/31 (in dt. Übersetzung auch in KRAFT 1974, 175-223) und GRÉGOIRE 1938. Sie kann als widerlegt gelten durch WINKELMANN 1962 (=WINKELMANN 1993, Nr. I), bes. 213-218 Referat der Position Grégoires (dort werden auch die zahlreichen weiteren Arbeiten des Verfassers zitiert und verarbeitet) und 242f. Widerlegung; wichtig auch 234-236, wo der Interpolationsverdacht im Blick auf die antipaganen Maßnahmen Konstantins im einzelnen diskutiert wird.

3 Vgl. mit weiteren Angaben WALLRAFF 2001b. Die Literatur zu Konstantin ist unübersehbar geworden. Ich nenne nur die wichtigsten monographischen Titel der vergangenen 15 Jahre: GRÜNEWALD 1990; BLEICKEN 1992; LeEB 1992; ClausS 1996; BleCKMANN 1996; LIEU MonTSERRAT 1998; DraKe 2000; MARCONE 2000; PiePENBrinK 2002. Nur am Rande wird hingegen die Religionspolitik Konstantins in der jüngsten Monographie über Euseb und das Heidentum behandelt, vgl. KOFSKY 2002, bes. 45-50 zu den hier interessierenden Schriften. 
Realität eliminiert werden. In Dokumenten aus Konstantins Kanzlei (auch sie freilich von Euseb überliefert, doch zweifellos unverfälscht!) konnte er etwa die Anweisung geben, „ohne allen Verzug sowohl alle Götzenbilder, die sich an dem genannten Orte vorfinden sollten, dem Feuer zu übergeben, als auch den Altar von Grund auf zu zerstören und, um es kurz zu sagen, alles derartige von dort vollständig verschwinden zu lassen und mit aller Kraft und auf alle Weise den gesamten Bezirk eifrigst zu reinigen". ${ }^{4}$ Der Ort, um den es geht, ist Mamre mit der heiligen Abrahamseiche - auch auf ihn komme ich gleich zurück.

Wie also verträgt sich dieser Konstantin mit demjenigen, der sich noch an seinem Lebensende nach seiner Taufe als zum Himmel fahrender Sonnengott auf seiner Konsekrationsmünze hat abbilden lassen? Dessen Statue in der von ihm gegründeten und nach ihm benannten Hauptstadt am Bosporus noch Generationen später religiös verehrt wurde? Diese Fragestellung ist von Euseb eigentlich ganz unabhängig, kommt aber angesichts der Quellenlage ohne ihn nicht aus, insbesondere ohne die von ihm geschilderten , antipaganen “ Maßnahmen.

Als letzte Vorbemerkung sei dieses auch im Titel genannte Schlagwort ,antipagan' kurz problematisiert. Daß die Begriffe ,Heidentum', ,pagan“ und demnach auch , antipagan' eine Verlegenheitslösung darstellen, bedarf gar keines Wortes, erst recht ist der häufig angebrachte Hinweis beinahe überflüssig, daß damit keine Wertung verbunden sein soll. Mehr als bei anderen Fragestellungen ist aber hier darauf hinzuweisen, daß die Gefahr nicht so sehr im pejorativen Beiklang liegt, sondern in der unzulässigen Kollektivierung. Im ,Heidentum wächst begrifflich zusammen, was sachlich keineswegs zusammengehört. Das „Heidentum‘ als geschlossene Größe gab es nicht - selbst für den voreingenommenen Euseb nicht. Er spricht bald von $\tau \grave{\alpha}$ है $\theta v \eta$, bald von oi "E und meint in beiden Fällen nur zum Teil Religiöses. ${ }^{5}$ Wir sind also gut beraten, wenn wir bei der Besprechung der antipaganen Maßnahmen möglichst sorgfältig differenzieren und darauf achten, welches Heidentum jeweils im Blick ist.

Ein erstes Beispiel ist die viel diskutierte Frage nach Konstantins Opferverbot. Euseb berichtet von einem diesbezüglichen Gesetz, ${ }^{6}$ doch hat diese

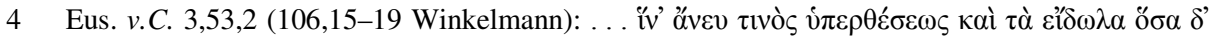

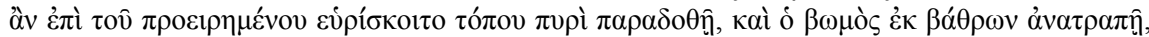

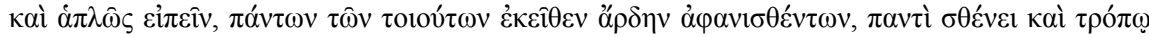

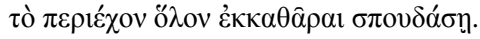

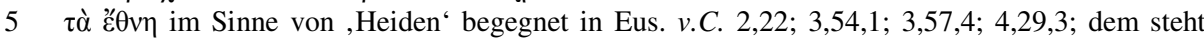
freilich eine viel größere Zahl von Belegen in anderen Bedeutungen gegenüber: 1,5,2; 1,7,1.2; $1,8,4 ; 1,14,2 ; 1,22,2 ; 1,25,1.2 ; 1,41,2 ; 1,48 ; 2,19,1 ; 2,44 ; 2,46,4 ; 3,1,5 ; 3,6,2 ; 3,29,2 ; 3,42,1$; $3,47,2 ; 3,50,2 ; 3,51,2 ; 3,59,1 ; 4,6 ; 4,14,1 ; 4,22,2 ; 4,27,2 ; 4,72$ (ein interessanter Grenzfall ist

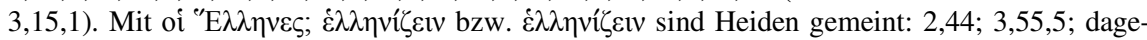
gen Griechen: $1,7,1 ; 2,23,1 ; 3,13,2 ; 4,8$.

6 Eus. v.C. 2,45,1. Über diesen Punkt hatte sich in der Folge von BARNES 1981, bes. $210 \mathrm{f}$. eine Kontroverse entsponnen, vgl. DRAKE 1982, bes. 464 f.; BARNES 1984; ERRINGTON 1988; BRADBURY 1994), vgl. auch CAMERON - HALl 1999, z.St. (245 f.). Als Resultat dieser Debatte läßt sich jedenfalls festhalten, daß ein so einseitig ,christlicher' Konstantin, wie ihn Barnes vor Augen stellt, schwerlich vorauszusetzen ist. Ich halte mich an die ausgewogene Darstellung von 
Mitteilung immer schon den Argwohn der Forscher erregt, zumal Euseb es wohlweislich unterläßt, den genauen Wortlaut mitzuteilen. Er spricht von dem Verbot der Weissagung und hängt gleichsam beiläufig an, daß es nun überhaupt verboten sei, Opfer darzubringen. Erhalten hat sich ein solches Gesetz nicht; erst unter Konstantins Söhnen bald nach dem Tod des Vaters ist eine solche Vorschrift mit Sicherheit erlassen worden (und im Codex Theodosianus erhalten). ${ }^{7}$ Freilich bezieht sie sich auf eine vorausgehende Bestimmung des Vaters. Daß es also etwas dergleichen gab, sollte man nicht bezweifeln, doch werden wir nie erfahren, was genau dort verboten wurde. Meines Erachtens wird man der komplexen Quellenlage am besten dadurch gerecht, daß man ein differenziertes Opferverbot annimmt, also etwa das Verbot, blutige Opfer im Kontext des staatlichen Kultes zwingend vorzuschreiben, dies vielleicht sogar nur in einem konkreten Streitfall. Mit einem solchen Erlaß wäre unmittelbar nach dem Sieg über Licinius ein wichtiges Signal an die Christen verbunden gewesen, die genau an dieser Stelle in der Verfolgungszeit stets in Konflikt gerieten. Es wäre gut erklärbar, daß Euseb pauschalisierend und übertreibend darin ein generelles Opferverbot erblickt, während der (nicht minder tendenziöse) Libanios Jahre später schreiben kann, daß Konstantin gegen Opfer nicht vorging. ${ }^{8}$

Sicherheit ist in dieser Frage nicht zu gewinnen, doch ist Konstantins Abneigung gegen blutige Opfer eine nicht zu bestreitende Tatsache. Aus dieser Tatsache ist - wenigstens teilweise - wohl auch die oben zitierte scharfe Ablehnung des in Mamre geübten Kultes zu verstehen. Das Beispiel ist in mehrfacher Hinsicht sehr lehrreich. Es geht um eine heilige Eiche, die in der jüdischen Tradition mit dem im Alten Testament $($ Gen 18,4) genannten Baum in Verbindung gebracht wurde, unter dem Abraham die drei Männer empfing, die ihn besuchten und ihm die göttliche Verheißung überbrachten. Schon diese Deutung des heiligen Baumes ist sicherlich sekundär, nicht nur weil in der Bibel an dieser Stelle keineswegs eine Eiche hervorgehoben genannt wird, sondern vor allem weil es ein paralleles ,Konkurrenzheiligtum' der Samaritaner in Sichem gab mit anderer Kultbegründung. Noch die kaiserzeitlichen Texte berichten Merkwürdiges über den jeweils geübten Kult: Die Bewohner der Gegend verehrten dort ihre Ahnen - erst spätere Zeugen verbessern in: „Patriarchen“ -, und sie

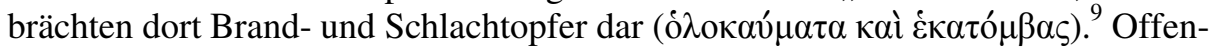

Bradbury, würde aber noch weitergehende Einschränkungen für Umfang und Tragweite der vermuteten gesetzlichen Regelung machen.

7 Cod. Theod. 16,10,2 (897 Mommsen/Krueger): Cesset superstitio, sacrificiorum aboleatur insania. Nam quicumque contra legem divi principis parentis nostri et hanc nostrae mansuetudinis iussionem ausus fuerit sacrificia celebrare, conpetens in eum vindicta et praesens sententia exeratur.

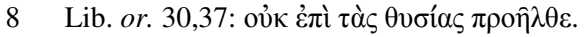

9 Afric. chronographiae frg. 18 (Routh). Eine (dringend erforderliche) Neuedition der Fragmente dieses Werkes wird von einer Gruppe unter meiner Leitung an der Universität Jena vorbereitet. Auf diese Edition muß ich auch mit Blick auf das textkritisch schwierige Stück über die 
sichtlich hatte also ein archaischer Baumkult überlebt. Die jeweiligen Orthodoxien hatten sich damit abgefunden und den Kult mehr schlecht als recht erträglich gemacht, indem sie ihm biblische Traditionen unterschoben. ${ }^{10}$ Nicht anders verfuhren die Christen, indem sie argumentierten, Mamre sei der Ort, an dem Christus zuerst in Menschengestalt erschienen sei, nämlich als einer der drei Engel bei Abraham. ${ }^{11}$

Weit über Christen und Juden hinaus zog der Ort die Menschen an; noch im fünften Jahrhundert muß es dort wahrhaft multireligiös zugegangen sein, schiedlich-friedlich teilten sich die Anhänger dreier religiöser Gruppen das Heiligtum - Zustände, wie man sie sich heute dort nur sehnlichst wünschen kann:

Auf das [dort begangene] Fest legen alle größten Wert: die Juden, weil sie stolz auf Abraham als ihren Patriarchen verweisen, die Heiden wegen der Erscheinung der Engel und die Christen, weil dort dem gottesfürchtigen Mann zuerst derjenige erschienen ist, der den Zeiten sich später selbst durch eine Jungfrau offen zeigte zum Heil des Menschengeschlechtes.

Der Religionswissenschaftler wäre beim Zusehen voll auf seine Kosten gekommen:

Die einen beten zu dem einen Gott des Alls, die anderen rufen die Engel an oder bringen Libationsopfer dar oder opfern Weihrauch oder einen Stier oder einen Ziegenbock oder ein Schaf oder einen Hahn. ${ }^{12}$

Kaum eine antike Kultpraxis fehlte! Wo so viel Heiligkeit versammelt war, durfte - damals wie heute - auch der Kommerz nicht fehlen; bei dem Heiligtum gab es einen großen Markt, auf dem man alles kaufen konnte, was zu den Riten erforderlich war und sicher noch vieles mehr; Käufer und Verkäufer, ob fromm oder nicht, gaben sich ein Stelldichein - ein exsecrabile mercatum, wie Hieronymus voll Abscheu sagt. ${ }^{13}$ Die anderen Beschreibungen stammen übrigens von Sozomenos, der all dies mehr als 100 Jahre nach Konstantin im verräterischen

Terebinthe in Sichem verweisen. Die Hauptzeugen des Textes sind Georgios Synkellos (123,13-21 Mosshammer) und (Ps.-)Eust. hex. (PG 18, 777D-780A).

10 In Sichem lokalisierte man den Baum, unter dem Jakob die Götterbilder vergrub, Gen 35,4. In Mamre bewirtete Abraham die Boten Gottes, Gen 18,1-15.

11 Just. dial. 56; Eus. h.e. 1,2,7f.; Eus. d.e. 5,9,7 (Text unten in Anm. 14).

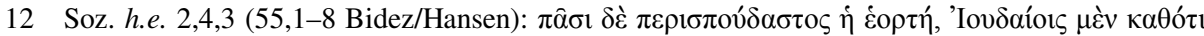

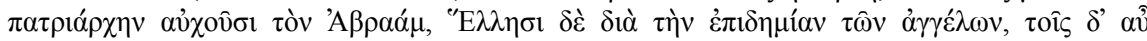
X

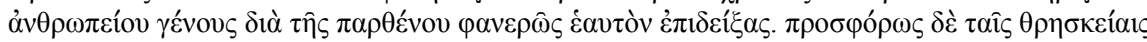

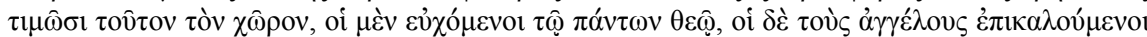

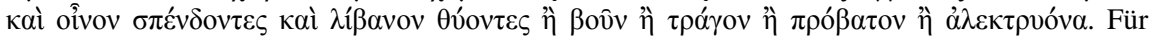
diesen multireligiösen Pilgerbetrieb hat sich ein außerordentlich interessantes nichtliterarisches Zeugnis erhalten: ein münzartiges Pilgerabzeichen, auf dessen Vorderseite die drei Engel bei Abraham unter der Eiche abgebildet sind und auf dessen Rückseite eine weibliche Gottheit ungeklärter Identität erscheint, vgl. FRAZER 1979, Nr. 522, 583 f. Ich danke Herrn Kollegen Dieter Korol (Münster), der mich bei der Tagung auf diesen Beleg aufmerksam machte.

13 Comm. in Ier. VI 18,6 und comm. in Sach. III 11,5. 
Präsens schreibt und - anders als Hieronymus - durchaus ohne abwertende Beiklänge, ganz im Gegenteil.

Dies wirft nicht nur ein interessantes Licht auf die Wirksamkeit - oder besser: Unwirksamkeit der Maßnahmen Konstantins (die von ihm gewollte und finanzierte christliche Basilika gab es nun, doch störte das die plurale Kulttradition nicht), sondern es zeigt auch, daß aus christlicher Sicht verschieden auf die Situation reagiert werden konnte. Pikanterweise hatte sich auch Euseb selbst einige Jahre vor dem Sieg Konstantins im Osten zu der Lokaltradition geäußert und dabei die Verehrung der Juden und Heiden für die heilige Eiche ganz irenisch als Zeichen der besonderen Würde und Bedeutung des Ortes der ersten Erscheinung Christi genommen, also im Sinne positiver Anknüpfung, nicht polemischer oder gar gewaltsamer Ablehnung. ${ }^{14}$ Konstantin also hat, wie wir in diesem Falle zeigen können, mit seiner Initiative weit über das hinausgegriffen, was die Christen (oder jedenfalls manche Christen) selbst für wünschenswert und erforderlich hielten. Er war sich dessen auch bewußt: das zeigt der in seinem Schreiben geäußerte Tadel den Bischöfen gegenüber, die dem heidnischen Greuel seit Jahren tatenlos zusahen. ${ }^{15}$ Euseb, einer der Adressaten des Briefes, steht zu diesem Tadel, zitiert das kaiserliche Schreiben in voller Länge und erspart sich eine weitere Stellungnahme.

An dieser Stelle wird deutlich, daß Motive und Stoßrichtung der antipaganen Tendenzen bei Euseb und Konstantin keineswegs die gleichen waren. Konstantin war in seiner Sicht dieses besonderen Falles vom Bericht seiner Schwiegermutter Eutropia bestimmt, die vor Ort gewesen war, ${ }^{16}$ und was ihn daran besonders abstieß, war der blutige Opferkult, ohne daß damit eine theoretische Begründung im Sinne einer Hochreligion verbunden war, kurzum: ihn störte das Archaische, das Un-Intellektuelle. Euseb hingegen, der Bischof vor Ort, sah eher die Möglichkeiten konstruktiver Anknüpfung und christlicher Mission.

Gerade umgekehrt liegen die Dinge bei der anderen bedeutenden christlichen Überbauung einer paganen Kultstätte im Heiligen Land, der Jerusalemer Grabeskirche. Auch dort erlaubt es uns die Quellenlage, die Motive Konstantins und die Eusebs nebeneinander zu halten. ${ }^{17}$ Beide erwähnen den paganen Kult, der sich über der Stätte des Grabes Christi etabliert hatte, doch während Konstantin mit wenigen Worten darüber hinweggeht, wird Euseb nicht müde zu betonen, wie schändlich und gottlos die Erbauer des Heiligtums gehandelt hatten, wie abscheuerregend der dort vollzogene Kult war und wie berechtigt seine

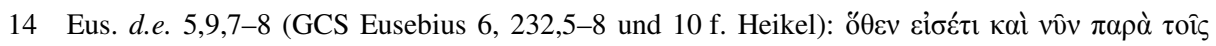

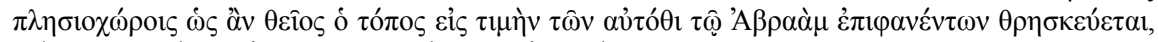

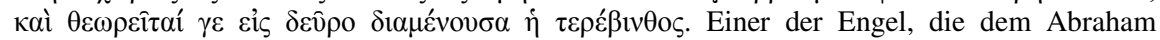

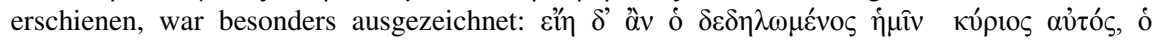

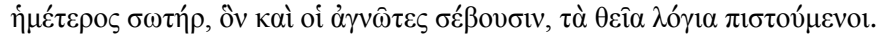

15 Eus. v.C. 3,52 f.

16 Das ergibt sich aus dem Beginn des Schreibens, v.C. 3,52.

17 Seine eigene Sicht gibt Euseb in v.C. 3,25-28; in v.C. 3,30-32 zitiert er das diesbezügliche Schreiben des Kaisers an Bischof Makarios. 
Zerstörung ist. Es ist der einzige nachweisbare Fall, in dem ein paganer Kult unterbunden, die entsprechende Kultstätte zerstört worden ist ausschließlich zugunsten des Christentums und ohne erkennbare andere Motive. Dies in Kombination mit dem hochgestimmten Überlegenheitsbewußtsein des Euseb wird man durchaus als Indiz dafür werten dürfen, daß sich auf christlicher Seite tatsächlich eine verbreitete und klare Vorstellung davon gehalten hatte, daß der Ort ,eigentlich' ihnen, den Christen gehörte, daß es ein primär christlich-heiliger Ort war und nicht umgekehrt wie in Mamre. Ohne nun in die Einzelheiten der kontroversen Echtheitsdiskussion in bezug auf das Grab Christi eintreten zu wollen, ${ }^{18}$ läßt sich doch festhalten, daß hier - und womöglich nur hier - den antipaganen Maßnahmen Konstantins dauernder Erfolg beschieden war. Die gewaltige christliche Kultanlage überdeckte die offenbar recht bescheidenen paganen Vorgänger vollständig und verdrängte den entsprechenden Kult. Tatsächlich mag es so gewesen sein, wie Euseb schreibt, ${ }^{19}$ daß das der Aphrodite geweihte Heiligtum von Anfang an seine raison d'être ausschließlich darin hatte, die christliche Grabtradition zu überdecken, also nicht auf alte und volkstümliche Wurzeln zurückging - entsprechend unkompliziert war dann seine Beseitigung.

Die Motivation Konstantins betreffend, tritt schon hier eher beiläufig ein Motiv auf, das sich noch mehrfach findet, nämlich die Tatsache, daß der Kaiser besonders am Aphrodite-Kult Anstoß nahm wegen der häufig damit verbundenen Kultpraxis der Tempelprostitution. Auch in Aphaka und in Heliopolis in Phönikien ist ein entsprechendes Vorgehen Konstantins belegt - Maßnahmen, die ebenso wie im Fall des Asklepiostempels in Aigai in der Darstellung Eusebs implizit oder explizit christlich motiviert erscheinen, ${ }^{20}$ doch sind dafür bei Lichte besehen keinerlei Beweise erkennbar. Selbst in Heliopolis/Baalbek nicht, obgleich Euseb dort im gleichen Atemzug die Gründung einer Kirche erwähnt. Erst bei genauer Lektüre stellt der Leser fest, daß von einer Überbauung keine Rede ist und daß beides - die Tempelschließung (nicht: Zerstörung!) und der Kirchbau - keineswegs zwingend in Zusammenhang stehen muß. ${ }^{21}$

18 Die umfangreiche Literatur zu Grab und Grabeskirche ist erschlossen über BIEBERSTEIN BLOEDHORN, 1994, 183-216, speziell zum Euseb-Text ferner die Angaben bei CAMERON HALL 1999, 285-289. Vgl. seither außerdem KRÜGER 2000.

19 Eus. v.C. 3,26,2.

20 Aphaka: Eus. v.C. 3,55; Heliopolis: v.C. 3,58; Aigai: v.C. 3,56. Es ist stets mit zu bedenken, daß neben religiösen auch ökonomische Motive bei Tempelschließungen eine Rolle gespielt haben das Vermögen fiel ja in diesem Fall an den Staat. Euseb deutet dies in l.C. 8,3 auch selbst an, vgl. dazu TARTAGLia 22001, 152, Anm. 130 und Maraval 2001, 129, Anm. z.St.

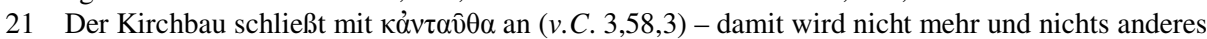
gemeint sein, als daß diese weitere Aktivität ebenfalls in Heliopolis stattfand; TARTAGLIA 22001 übersetzt daher sinngemäß zutreffend mit ,e anche nella loro città“. Die Forschung ist bis in die neueste Zeit der Suggestivkraft der Eusebschen Darstellung erlegen, so wenn KoCH 1998, Sp. 1040 f. mit Berufung auf Euseb sagt, daß „Konstantin einen Aphrodite-Tempel abreißen und an seiner Stelle eine Kirche errichten“ ließ. Nichts davon steht im Text! Die konstantinische Kirche ist archäologisch nicht nachgewiesen (КоCH 1998 ebd.). Sehr viel klarer werden die Verhältnisse übrigens in $l . C$. 8,5-7, weil dort vom Kirchbau gar nicht die Rede ist, dafür aber 
Man konnte jedenfalls ohne weiteres an den genannten Formen der Kultpraxis Anstoß nehmen, ohne überzeugter Christ zu sein (ebenso wie übrigens in einem weiteren, ähnlich gelagerten Fall in Ägypten ${ }^{22}$, und es ist umgekehrt keineswegs ausgemacht, daß die vereinzelten Maßnahmen Konstantins gegen gewisse Kulte allgemein als Signal zugunsten des Christentums verstanden wurden. Im Gegenteil spricht manches dafür, daß Konstantin eine Art christlich geläutertes Heidentum als Staatsreligion vorschwebte, gewissermaßen eine Hegelsche Synthese der jeweils besten Anteile der unterschiedlichen religiösen Traditionen des Reiches - eine Synthese, die vermutlich schon unter den Zeitgenossen niemand wirklich verstand und niemand wirklich wollte.

Das Programm läßt sich besonders gut am Profil der neu gegründeten Hauptstadt des Ostens am Bosporus ablesen. ${ }^{23}$ Euseb hatte davon vergleichsweise oberflächliche Kenntnis, viel weniger tiefgehend natürlich als in den bisher genannten Fällen aus Palästina und Umgebung, auf die sich im übrigen seine Darstellung weitgehend konzentriert. Schon eingangs hatten wir gehört, daß Konstantin die „,verehrten Erzbilder, deren sich der Trug der Alten so lange Zeit gerühmt hatte, auf allen Plätzen der Kaiserstadt offen ausgestellt [habe], damit sie allen, die sie sahen, einen abstoßenden Anblick böten, ... im Hippodrom die delphischen Dreifüße und die Musen des Helikon im Palast “24. Es ist eher unwahrscheinlich, daß der Kaiser kostbare Objekte von weither kommen ließ in der Absicht, daß sie dann in seiner neuen Hauptstadt einen abstoßenden Anblick böten. Über viele Jahrhunderte bildete die Blütenlese antiker Kunst im repräsentativen Zentrum der Hauptstadt das Herz des byzantinischen Reiches; die Schlangensäule, die den delphischen Dreifuß trug, ist übrigens bis heute im Hippodrom zu sehen. ${ }^{25}$ Auch wenn Euseb sagt, , daß der Kaiser aus diesen [Götzenbildern] für alle, die sie sehen wollten, ein Spielzeug machte, das dem Gelächter und Spott diente ${ }^{\text {‘26 }}$, so handelt es sich um eine groteske Verzeichnung der kaiserlichen Intentionen.

Wahr ist, daß viele der aufgestellten Kunstwerke ihres ursprünglichen Kontextes und damit vielfach auch ihrer ursprünglichen religiösen Valenz beraubt

deutlich wird, daß der pagane Tempel keineswegs in der Stadt lag, sondern weit abgelegen außerhalb.

22 Eus. v.C. 4,25 (Verbot der entmannten Nilpriester).

23 Vgl. DAGRON 1974, 19-47; MANGO 1990a, 23-36.

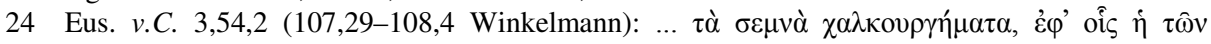

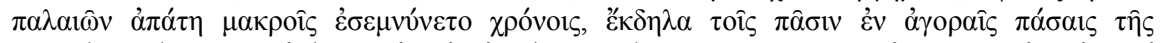

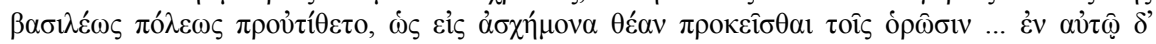

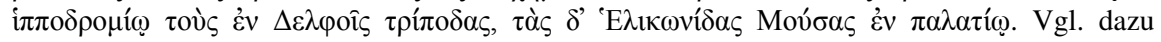
CAMERON - HALL 1999, z.St. (301 f.).

25 Eine vermutlich realistischere Wiedergabe der Intentionen Konstantins bietet Zos. 2,31, wichtige Informationen auch bei Jo. Mal. chron. 13,13 (248,56-59 Thurn). Zu der einzigartigen Antikensammlung im Hippodrom, auch zu ihrer Bedeutung im imperialen Programm der Hauptstadt vgl. BASSETT 1991, speziell zu der Schlangensäule 90.

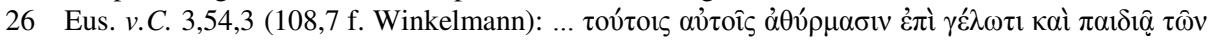

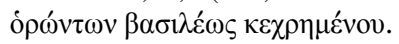


waren. $\mathrm{Da} ß$ damit jedoch die Absicht verbunden war, sie lächerlich zu machen, wird schwerlich der Fall gewesen sein. Vielmehr wollte sie Konstantin wenigstens teilweise - einer neuen, höheren religiösen Aussage zuführen. Vielleicht nicht im Hippodrom, aber doch im nahe gelegenen Heiligtum der Weisheit, später Hagia Sophia genannt; dort jedenfalls läßt sich so etwas noch schemenhaft aus den Quellen erheben. ${ }^{27}$ Noch in justinianischer Zeit standen dort Statuen diverser Gestirngottheiten, darunter auch der zwölf Zodia - Symbole einer abstrakt-intellektuellen Astralreligion, wie sie damals en vogue war. Wenigstens vermutungsweise läßt sich aus dem Befund ablesen, daß Konstantin damit eine Art Astralheiligtum schaffen wollte und daß daher die SophienKultstätte zunächst nicht die christliche бoøía etwa im Sinne einer Christusmetapher im Blick hatte, sondern schlicht die Weisheit des Kaisers. Tatsächlich ist von einer christlichen Kirche und Kirchweihe an dieser Stelle erst unter Konstantins Sohn Konstantios die Rede.

Wenn dem so war, ist allerdings schwer erklärbar, wieso Euseb von einem Gesetz berichtet, mit dem Konstantin den Kaiserkult verboten habe. ${ }^{28}$ Mit dieser Information ist jedoch auch schon schwer vereinbar, daß noch um 330 in Hispellum in Umbrien ein Tempel für die gens Flavia mit ausdrücklicher Billigung des Kaisers neu errichtet wurde, wobei allerdings contagiosa superstitio unterbleiben sollte. ${ }^{29}$ Ähnlich wie beim Opferverbot ist das Problem wiederum mit der Annahme einer partiellen Wahrheit zu lösen. Es ist gut möglich, daß Konstantin blutige Opfer im Angesicht seines Bildes tatsächlich unterbinden wollte. Daß ihm die Verherrlichung seiner Person und damit verbundene kultische Konsequenzen keineswegs grundsätzlich zuwider waren, zeigt die bestens bezeugte und teilweise noch heute vorhandene Porphyrsäule mitten in Konstantinopel, aufgestellt am höchsten Punkt der Stadt mit weitem Blick über zwei Meere und zwei Kontinente, auf deren Spitze sich Konstantin als Sonnengott Helios stilisiert darstellen ließ.$^{30}$ Noch das fünfte Jahrhundert, christianissima tempora, kannte dort kultische Verehrung des Stadtgründers durch die Bevölkerung - eine Verehrung, die den Intentionen des Erbauers schwerlich ganz zuwider lief. ${ }^{31}$ Dieselbe eigenartige Verbindung von geläutertem Heidentum und imperialer Selbstverherrlichung ließe sich auch an der sogenannten Apostelkirche zeigen, für die Euseb sich teilweise etwas mühselig eine interpretatio christiana

27 WALLRAFF 2006.

28 Eus. v.C. 4,16.

29 CIL 11,5265 = ILS $705:$ :... ne aedis nostro nomini dedicata cuiusquam contagiose superstitionis fraudibus polluatur.

30 Vgl. Theodor PReger 1901, KARAYANNOPUlos 1956 (auch in WlosoK 1978, 485-508); DAGRON 1974, 38 f.; BERGER 1988, 297-299; SPECK 1995, 160-168; BERGMANN 1998, 284 287; WALLRAFF 2001a, $133 \mathrm{f}$.

31 Philostorgios berichtet, daß ,die Christen das Bild Konstantins, das auf der Porphyrsäule steht, durch Opfer geneigt zu machen versuchen, es mit Räucherwerk und Lichtern ehren, ihm Gebete darbringen wie einem Gott und Schutzriten zur Abwendung von Unheil vollziehen." Philost. h.e. 2,17 (GCS 28,4-7 Bidez/Winkelmann). 
zurechtlegt, ohne doch andererseits die naheliegende Konsequenz einer Konstantin-Christus-Typologie zu ziehen, obgleich sie der Erbauer vermutlich selbst beabsichtigte. ${ }^{32}$

Ich breche hier $a b$, auch wenn es außerordentlich reizvoll wäre, die spezifische Form von Konstantins Religiosität eingehender zu rekonstruieren, einen Frömmigkeitstypus, den man in einem höheren Sinn als HeidenChristentum bezeichnen könnte. Hierfür ist trotz der reichen Forschungsliteratur zu Konstantin auch aus jüngster Zeit das letzte Wort sicher noch nicht gesprochen. Im Hinblick auf die Quellenlage ist dabei zu beachten, daß die Überlieferung stark selektiv im Sinne späterer christlicher Orthodoxie gewirkt hat. Diese Feststellung ist nicht neu und nicht überraschend, doch sind die Konsequenzen häufig nicht hinreichend bedacht. Es bedeutet etwa, daß Schriftstücke aus Konstantins Kanzlei dezidiert nicht-christlichen Charakters eine erheblich schlechtere Überlieferungschance hatten als die Urkunden, die Euseb überliefert, gewissermaßen den Ausstoß des Referats für christliche Angelegenheiten in der Zentralverwaltung. Es bedeutet auch, daß die wenigen Indizien für den , unorthodoxen ' Konstantin (ich sage bewußt nicht einfach: ,heidnischen') um so schwerer wiegen und um so sorgfältiger betrachtet und interpretiert werden müssen. Ein vages Indiz dieser Art kommt in seinem Gewicht angesichts der Überlieferungsbedingungen und -chancen zehn klaren Informationen über das Christentum des Kaisers gleich. Und es bedeutet schließlich, daß damit ein Lektüreschlüssel für die antipaganen Maßnahmen Konstantins in der Darstellung Eusebs gegeben ist. Die Nachrichten darüber sind nicht einfach wertlos, auch wenn Skepsis natürlich angebracht ist. Daß sie, wenn irgend möglich, mit anderen Informationen geprüft werden sollten, ist eine Selbstverständlichkeit. Wichtiger ist, daß sie - bei kritischer Lektüre im Lichte der nichtchristlichen Kulttraditionen - erstaunlich viel verraten über die spezifischen Gestalten von ,Heidentum', wie sie Konstantin ablehnte und anstrebte: blutigen Opferdienst, archaische Baumkulte, sexuell ausschweifende Kultpraxis, wie er sie ablehnte; inklusiven Monotheismus (das Christentum natürlich mit umfassend), intellektuell-abstrakte Gestirnverehrung und römisch-loyalen Staatskult, wie er sie anstrebte.

$\mathrm{Da} ß$ diese ingeniöse Kombination keine Zukunft hatte, wissen wir heute mit der einfachen Klugheit der nachgeborenen Historiker. Unter den Zeitgenossen sah

32 Wie sich die Elemente Apostelmemoria, christlicher Gottesdienst und kaiserliche Grablege zueinander verhielten, bleibt in v.C. 4,60 durchaus unklar - oder vielmehr: es wird deutlich, daß (nur) in der Sicht des christlichen Betrachters das kirchliche Element zunächst vorherrschte.

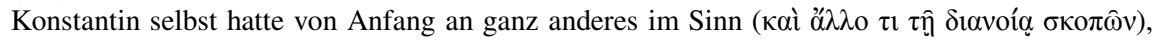
eben die Erhöhung des verstorbenen Kaisers im Kreise, ja: im Zentrum der Apostel (wenn die zwölf Stelen überhaupt so eindeutig christlich gedeutet werden müssen - ein anderer Betrachter konnte womöglich auch die zwölf Zodia darin erblicken, in deren Zentrum dann strahlend Helios, der „Sonnenkönig“, stände, wie im Falle der Statue), vgl. WALLRAFF 2001a, 135 f. Die Apostelkirche ist in jüngerer Zeit wieder intensiver diskutiert worden, vgl. vor allem MANGO 1990b (im Hinblick auf die Baugestalt grundlegend); LEEB 1992, 93-120; SPECK 1995, 144 f.; REBENICH 2000, 309-317; EFFENBERGER 2000. 
es vielleicht keiner so klar wie Euseb. Konstantin wurde ironischerweise dennoch zu einem der Großen der Geschichte - und zwar weil die konfrontativapologetische Lektüre seiner Religionspolitik, wie sie offenbar auch möglich war und wie sie bei Euseb vorliegt, sehr wohl Zukunft hatte. Systematische Maßnahmen zur Ausschaltung des Heidentums mit Unterstützung der öffentlichen Verwaltung sollten in den kommenden Jahrzehnten zum Regelfall werden.

\section{Literatur}

Barnes, T.D., Constantine and Eusebius, Cambridge, MA 1981.

Barnes, T.D., Constantine's Prohibition of Pagan Sacrifice, AJPh 105 (1984), 69-72.

Bassett, S.G., The Antiquities in the Hippodrome of Constantinople, DOP 45 (1991), 87 96.

Berger, A., Untersuchungen zu den Patria Konstantinupoleos (ПOIKI $\Lambda$ A BYZANTINA 8), Bonn 1988.

Bergmann, M., Die Strahlen der Herrscher. Theomorphes Herrscherbild und politische Symbolik im Hellenismus und in der römischen Kaiserzeit, Mainz 1998.

Bieberstein, K. - Bloedhorn, H., Jerusalem. Grundzüge der Baugeschichte vom Chalkolithikum bis zur Frühzeit der osmanischen Herrschaft, Bd. II (Tübinger Atlas des Vorderen Orients, Beiheft B 100/1-3), Wiesbaden 1994.

Bigelmair, A., Des Eusebius Pamphili, Bischofs von Cäsarea, ausgewählte Schriften (BKV 9), Kempten 1913.

Bleckmann, B., Konstantin der Große, Reinbek 1996.

Bleicken, J., Constantin der Große und die Christen (HZ Beiheft 15), München 1992.

Bradbury, S., Constantine and the Problem of Anti-Pagan Legislation in the Fourth Century, CPh 89 (1994), 120-139.

Cameron, A. - Hall, S.G. (Hrsg.), Eusebius, Life of Constantine, Oxford 1999.

Clauss, M., Konstantin der Große und seine Zeit, München 1996.

Dagron, G., Naissance d'une Capitale. Constantinople et ses institutions de 330 à 451, Paris 1974.

Drake, H.A., Rez. zu BARNES 1981, AJPh 103 (1982), 462-466.

Drake, H.A., Constantine and the Bishops: The Politics of Intolerance, Baltimore 2000.

Effenberger, A., Konstantinsmausoleum, Apostelkirche - und kein Ende? in: Borkopp, B.

- Steppan, T. (Hrsg.), $\Lambda \mathrm{I} \Theta \mathrm{O} \Sigma \mathrm{TP} \Omega \mathrm{TON}$. Studien zur byzantinischen Kunst und Geschichte. Festschrift für Marcell Restle, Stuttgart 2000, 67-78.

Errington, M.R., Constantine and the Pagans, GRBS 29 (1988), 309-318.

Eusebius, Laus Constantini, hrsg. v. I.A. Heikel (GCS Eusebius 1), Berlin 1902, 195-223.

Frazer, M.E., Mold with Three Angels at Mamre, in: Weitzmann, K. (Hrsg.), Age of Spirituality: Late Antique and Early Christian Art, Third to Seventh Century, New York 1979, Nr. 522, 583.

Grégoire, H., La ,conversion` de Constantin, RUB 36 (1930-31), 231-272.

Grégoire, H., Eusèbe n'est pas l'auteur de la ,Vita Constantini ' dans sa forme actuelle et Constantin ne s'est pas ,converti` en 312, Byzantion 13 (1938), 561-583.

Grégoire, H., La ,conversion` de Constantin (dt. Übersetzung), in: Kraft, H. (Hrsg.), Konstantin der Große (Wege der Forschung 131), Darmstadt 1974, 175-223. 
Grünewald, T., Constantinus Maximus Augustus. Herrschaftspropaganda in der zeitgenössischen Überlieferung (Historia Einzelschriften 64), Stuttgart 1990.

Heikel, I. (Hrsg.), Über das Leben Constantins (GCS Eusebius 1), Berlin 1902.

Karayannopulos, I., Konstantin der Große und der Kaiserkult, Historia 5 (1956), 341-357.

Koch, G., Baalbek, in: RGG 1 (1998), 1040f.

Kofsky, A., Eusebius of Caesarea against Paganism (Jewish and Christian Perspectives 3), Leiden 2002.

Krüger, J. Die Grabeskirche zu Jerusalem. Geschichte - Gestalt - Bedeutung, Regensburg 2000.

Leeb, R., Konstantin und Christus. Die Verchristlichung der imperialen Repräsentation unter Konstantin dem Großen als Spiegel seiner Kirchenpolitik und seines Selbstverständnisses als christlicher Kaiser (AKG 58), Berlin 1992.

Lieu, S.N.C. - Montserrat, D. (Hrsg.), Constantine : History, Historiography and Legend, London 1998.

Mango, C., Le développement urbain de Constantinople (IV ${ }^{\mathrm{e}}-\mathrm{VII}{ }^{\mathrm{e}}$ siècles), Paris ${ }^{2} 1990$ a, 23-36.

Mango, C., Constantine's Mausoleum and the Translation of Relics, ByzZ 83 (1990b), 51-62.

Maraval, P., Eusèbe de Césarée. La théologie politique de l'empire chrétien. Louanges de Constantin (Triakontaétérikos), Paris 2001.

Marcone, A., Costantino il Grande, Rom 2000.

Piepenbrink, K., Konstantin der Große und seine Zeit, Darmstadt 2002.

Preger, T., Konstantin-Helios, Hermes 36 (1901), 457-469.

Rebenich, S., Vom dreizehnten Gott zum dreizehnten Apostel? Der tote Kaiser in der Spätantike, Zeitschrift für antikes Christentum 4 (2000), 300-324.

Speck, P., Urbs, quam Deo donavimus. Konstantins Konzept für Konstantinopel, Boreas 18 (1995), 143-173.

Tartaglia, L., Eusebio di Cesarea, Sulla Vita di Costantino (Quaderni di KOIN@NIA 8), Neapel ${ }^{2} 2001$.

Wallraff, M., Christus Verus Sol. Sonnenverehrung und Christentum in der Spätantike (JbAC Ergänzungsband 32), Münster 2001a.

Wallraff, M., Constantine's Devotion to the Sun after 324, in: Wiles, M.F. - Yarnold, E.J. (Hrsg.), StudPatr 34 (2001b), 256-269.

Wallraff, M., Gab es eine konstantinische Hagia Sophia in Konstantinopel? in: Harreither, R. u. a. (Hrsg.), Akten des XIV. Internationalen Kongresses für Christliche Archäologie, Wien 19.-26.9.1999. Frühes Christentum zwischen Rom und Konstantinopel (SAC 62 = ÖAW.PH Arch. Forsch. 14), Rom - Wien 2006, 767-774.

Winkelmann, F., Zur Geschichte des Authentizitätsproblems der Vita Constantini, Klio 40 (1962), 187-243.

Winkelmann, F., Über das Leben des Kaisers Konstantin (GCS Eusebius 1,1), Berlin ${ }^{2} 1975$.

Winkelmann, F., Studien zu Konstantin dem Großen und zur byzantinischen Kirchengeschichte, Birmingham 1993, Nr. I.

Wlosok, A. (Hrsg.), Römischer Kaiserkult (Wege der Forschung 372), Darmstadt 1978. 


\title{
The Imperial Cult in Late Roman Religion (ca. A.D. 244-395): Observations on the Epigraphy
}

\author{
FRANK R. TROMBLEY
}

The imperial cult was a conventional feature of urban political and religious life in the later Roman empire. It was observed continuously throughout the politically troubled era of the usurpers in the later third century, and afterward under the Tetrarchy and Constantinian, Valentinian and Theodosian dynasties. A considerable number of historical texts and inscriptions indicate the status of the imperial cult between two significant dates, A.D. 244, when Philip the Arab was raised to the imperium, and the death of Theodosius the Great A.D. 395. ${ }^{1}$ This period is less well documented than the preceding one as regards temples of the imperial cult, its priesthoods and the ritual practices entailed in it. One Christian writer, Eusebius of Caesarea, promoted the view that Philip the Arab had been the first Christian emperor, a proposition that has been exhaustively analysed in recent decades. ${ }^{2}$ Whether this was the case or not, there were few changes in the way the imperial cult was practised until after the death of Theodosius the Great, as evidenced in the epigraphy and the laws in the Theodosian and Justinian Codes. ${ }^{3}$

The view is sometimes expressed that Theodosius the Great made Christianity the 'official' religion of the later Roman empire, particularly through the crucial law he issued on 8 November $392 .^{4}$ This is a doubtful proposition. The decisive historical moment undoubtedly came later, with the comprehensive legislation of Justinian the Great ca. 529-534. The period between 244-395 saw direct continuity in the observance of the imperial cult. The scepticism expressed by scholars about the persistence of this political and theological reality is a consequence of their being influenced by polemics of Christian writers like Origen, who led a life largely divorced from active participation in civic life or in the administration of the Roman state, and could therefore afford to entertain dissi-

1 The earlier bibliography is conveniently summarised in BONAMENTE 1988, 107-142. Comprehensive studies include BEURLIER 1891, 283-300, 328-331, 343-346. See also BOWERSOCK 1982, 171-182, 238-241 and MACMULLEN 1997, 35f. I wish to thank Angela Amici for copies of her two studies AMICI 2000a, 187-216 and AMICI 2000b, 13-55.

2 E.g. SHAHÎD 1984, 65-93, etc.

3 See broadly: PRICE 1984; GRADEL 2002.

4 This point was made in TROMBLEY 1993-94, 1:13-17. The argument is more fully developed in ERRINGTON 1997a and especially ERRINGTON 1997b. LIZZI TESTA 1996. 
dent opinions that only obliquely acknowledged the importance of the imperial cult. There is a surprisingly large amount of evidence on this for the period in question. In what follows, some of the more significant texts will be identified and investigated, where the emphasis will be on the persistence of the Senate's conferral of the title divus, the personnel of the priesthood, temples and the other accessories of cult, and the Nachleben of the imperial cult under the Christian empire (ca. A.D. 312-534).

\section{Honouring Emperors: Imperial Cult ca. A.D. 244-305}

The numerous assassinations of emperors during the crisis of the mid-third century did not affect the continuity of the imperial cult. ${ }^{5}$ Usurpers seem frequently to have urged the Senate to apply a process called damnatio memoriae to their predecessors as a tool to promote the legitimacy of their usurpation. ${ }^{6}$ It involved annulling the edicts and destroying the statuary and inscriptions of a 'bad' emperor after his deposition; it would presumably have also involved re-minting coins bearing the latter's image. The process was of course fraught with all kinds of practical difficulties. The erasure of emperors' names is common in the epigraphy of the third century, but most of the inscriptions even of condemned emperors seem to have been left untouched, as a perusal of the epigraphic collections reveals. Although it is strictly speaking impossible to date the political circumstances of particular erasures, they seem normally to have been done after a decree of damnatio memoriae was issued by the Senate; there are instances, however, where provincial governors seem to have acted on their own initiative, as in a case of the erasure of Aurelian's name. ${ }^{7}$

It is a natural corollary that the images of the condemned emperors would have been removed from the temples of the imperial cult in towns where their names were erased from the inscriptions. After this, the images might be either smashed into small fragments, or reworked into sculptures depicting their successors. ${ }^{8}$ Thus, in the politically difficult years between 244 and 284, the images of new emperors were constantly being installed in shrines while those of condemned emperors were being discarded. So, for example, the same official, a certain Aurelius Iulius who was equestrian governor of the province of Baetica, sponsored a dedication to emperor Florianus, who reigned for a about a year in

5 On the period, see DRINKWATER 2005.

6 ELSNER 1998, 55-57. GIZEWSKI 2004. The provocative question of whether the erasures found in inscriptions of ca. 244-312 do in fact confirm acts of damnatio memoriae against the emperors of this period is not directly addressed in this article.

7 See below, n. 19.

8 Cf. statues of Augustus and Livia in the Archaeological Museum of Ephesus. SCHERRER 2000, 214-215. 
A.D. 276, and then another to Probus, the latter's immediate successor. ${ }^{9}$ Thus, political conditions were so unstable and turnovers in the imperial office so frequent that a governor's term in office might extend across the reign of two or more emperors. ${ }^{10}$ The statues of some of the latter nevertheless survive, among them the famous image of Philip the Arab in the Vatican Museum, as well as those of Trebonius Gallus, Gallienus, Carinus and possibly Numerianus. ${ }^{11}$ It is impossible to say if these portraits were originally installed in temples of the imperial cult or belonged to other honorific contexts.

The names and titles most frequently erased from inscriptions during this period ca. A.D. 244-284 seem to have been those of Philip the Arab and his son and co-emperor Caesar M. Iulius Philippus, ${ }^{12}$ but this phenomenon is apparent also in the inscriptions of Decius, Trebonianus Gallus, Gallienus and his son Caesar P. Licinius Saloninus, Gallienus' brother Caesar Valerian, and Aurelian. ${ }^{13}$ At the end of this period many inscriptions had the names of Carus and his sons the Caesars Carinus and Numerianus erased; ${ }^{14}$ the circumstances surrounding the erasures of other emperors' names except Philip, Trebonianus Gallus and Gallienus suggest that Carus and his sons were probably identified as 'bad' emperors and therefore appear not to have been deified. The possible damnatio memoriae of these men was unquestionably pursuant to an initiative of Diocletian who was anxious to consolidate his position with the Senate and army after the overthrow of a legitimate regime. ${ }^{15}$

In some instances, names of deified emperors were erased, among them Caesar Valerianus, who had been co-emperor with his father Valerian and brother Gallienus (divo Valeriano Caes(ari) Cinguliani d.d.p.) and whose consecratio was also commemorated on coins. ${ }^{16}$ Separate inscriptions at the provincial town of Cingulum in Picenum indicate the erasure of the names of both Gallienus' sons, Saloninus and divus Caesar Valerianus. ${ }^{17}$ This suggests that the latter had died already, before Gallienus was assassinated in 268, and that his brother's persuasion (or coercion) was responsible for the younger Valerian's deification. It is peculiar that the people of Cingulum were so punctual in erasing the two Caesars' names when so many other inscriptions of theirs have survived without being defaced. Of the other emperors, Claudius Gothicus is known to have been deified from his consecration coins and from an inscription at Thamugadi in $\mathrm{Nu}-$ midia. ${ }^{18}$ In an inscription at Thuburuscum, also in Numidia, Emperor Aurelian

$9 \quad$ ILS 579; CIL III 7586.

10 ILS $593=$ CIL II 1115-1116.

11 SHAHID 1984, frontispiece. GRANT 1968, pls. 14-18, 22-23.

12 E.g. ILS 505 (Rome), 508 (Henchir Naam, Africa), 509 (Galleria de Furlo, Umbria).

13 ILS 518, 526, 545, 547, 556, 558, 579, 597.

14 ILS 599, 601, 603, 605-608.

15 See H. Dessau's remark at ILS 608.

$16 I L S 556=C I L$ IX 5682; ILS $557=C I L$ VIII 8473

17 ILS 558, 559.

18 ILS 572. 
was referred to as a god while still alive (deo Aureliano $r(e s)$ p(ublica) $T(h) u\left(\right.$ burisicitanae)), a characteristic of his coins as well. ${ }^{19}$ It is not entirely clear why Aurelian's name is erased from some inscriptions, one of them at Brixia (present-day Brescia), the other in Moesia Inferior, except that local military commanders or civil governors acted on their own initiative before receiving clear instructions. ${ }^{20}$ The deification of Carus undoubtedly took place with the generous consent of the Senate, and effectively conferred legitimacy on his sons Carinus and Numerianus, who had first been promoted to the rank of Caesars and then Augusti. ${ }^{21}$ A temple of the imperial cult was constructed in their honour at Verecundia near the camp of the III Augusta legion at Lambaesis in Numidia, according to a badly damaged inscription: ${ }^{22}$

For the preservation of the most fortunate emperors, our lords Carinus and Numerianus, the town (res publica municipium) of the Verecundensians constructed a temple from the foundations up in honour of their father, the deified Carus ( . . . [divi Cari] genitoris eorum tem[plum a fundamentis] . . consti[tuit]).

The immediate consecratio of Carus is quite likely in view of his sons' immediate succession and the fact that his grandson (nepos) Nigrianus is also called divus in an inscription in the Forum of Augustus in Rome. ${ }^{23}$ The Lambaesis inscription implicitly makes the point that the new temple and its cult would be conducive to the welfare of the two sons of a deified father. As things turned out, the city councillors were mistaken.

The accession of Diocletian in 284 and subsequently that of his colleagues put an end to the consecratio and installation of emperors for two decades. This was a consequence of the longevity of the emperors of the Tetrarchy, the first of them to die being Constantius I over two decades later, on 25 July 306. Dedications to the numen of Diocletian and his colleagues are numerous. ${ }^{24}$ A salute to the genii of the two branches of his regime, the Iovian and the Herculean, is found in an inscription found near the theatre of Pompeius in Rome: ${ }^{25}$

To the genius of the Iovian Augustus, after the work on his Iovian portico was completed and ornamented from the foundations up. Aelius Dionysius vir clarissimus (presided over) the task of completing the work.

To the genius of the Herculean Augustus, after the work on the Herculean portico was completed and ornamented from the foundations up. Aelius Dionysius vir clarissimus (presided over) the task of completing the work.

Divine parenting was also attributed to Diocletian and Maximianus the Elder. Although these two emperors originated from the officer class of Illyricum, an

19 ILS 585.

$20 I L S 579=$ CIL V 4319 (A.D. 274), CIL III 7586.

21 ILS 608. PARKER 1958, 219-220.

22 ILS 609 = CIL VIII 4221.

23 ILS 611.

24 E.g. ILS 614, etc.

$25 I L S 621$ and $622=C I L$ VI 254 and 255. 
inscription at Dyrrachium describes their children as descended from gods. The question remains open as to whether the co-emperors were seen as living gods, as Aurelian had claimed, or as men who were certain to be deified because of their great achievements in reconstructing the empire and the soundness of their character: ${ }^{26}$

To our lords Diocletian and Maximianus, the unconquered Augusti, who were begotten by gods and are the begetters of gods (diis genitis et deorum creatoribus) [---].

The inscription dates between 285, when Maximianus was appointed Caesar, and 293, when Galerius and Constantius I joined the college of emperors to form the Tetrarchy. The 'divine' children in question included among others Valeria daughter of Diocletian and Maxentius son of Maximianus. The mentality expressed in the inscription is consistent with the allusion of an anonymous panegyrist writing in A.D. 291 to the divine parentage of Maximianus Herculeus (siquidem vos dis esse genitos ...). ${ }^{27}$ It would be valuable to know what innovations of cult, ritual and iconography, if any, accompanied the implementation of these conceptions.

The names of the Tetrarchs were erased from their inscriptions in many towns sometime after Constantine became emperor. ${ }^{28}$ The statues of Diocletian and Maximianus Herculeus were also removed from places of honour ca. 311. Lactantius describes the damnatio memoriae in these terms: ${ }^{29}$

At the same time the statues of the elder Maximian were being thrown down on the orders of Constantine, and any pictures in which he had been portrayed were being removed. And because the two old men had been painted (picti) together, this meant that the pictures of both were being taken down. Diocletian thus saw happening to him in his own lifetime what had never happened to an emperor before.

Diocletian's bewilderment is explained by the fact that the condemnation of an emperor normally took place after his death, but that he was being subjected to de facto damnatio memoriae through his association with the elder Maximianus. Paintings of this type have not survived, but their character may be surmised from the great porphyry image of the Tetrarchs holding each other in a fraternal embrace that is built into San Marco cathedral in Venice. ${ }^{30}$ Eusebius makes it clear that it was Maximianus against whom the action was directed, and that it was taken after his death: ${ }^{31}$

26 ILS 629 = CIL III 710.

27 Paneg. XI (III) 2.4. See RODGERS 1986, 77-78.

28 See H. Dessau's comment at ILS 630.

29 Lact. mort. pers. 42 (quotations are from the edition and translation by CREED 1984).

30 ELSNER 1998, 62.

31 Eus. h. e. 8.13 . 
He was the first whose honorific inscriptions, statues and everything else that it

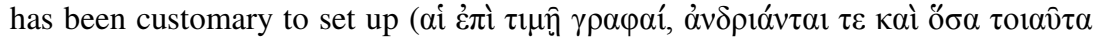
$\left.\varepsilon^{\prime} \pi{ }^{\prime} \alpha \alpha_{\alpha} \theta \varepsilon \sigma \varepsilon l v \varepsilon v o ́ \mu 1 \sigma \tau \alpha l\right)$, were destroyed as reminders of an unholy and impious man.

This could have been done only in the regions that Constantine controlled, including Gaul, Britain and Spain, but excluding Italy and Africa. This being the case, the erasures from some inscriptions must have occurred later, as for example at Verona, Thamugadi in Numidia and Henchir Midid in Proconsular Africa. ${ }^{32}$ It should probably be assumed from this that Constantine also initiated a programme of removing statues of Maximianus from shrines of the imperial cult and smashing them. It is probable that Maximianus was nevertheless deified by the Senate in Rome at the behest of his son Maxentius, who was ruling Italy and Africa outside the recognised college of emperors. As will be seen below, at least one of the sons of Constantine effectively rescinded the damnatio memoriae of their grandfather and retroactively recognised Maximianus Herculeus as divus. The erasure of his name and that of Diocletian in the eastern provinces came much later, probably after Constantine became sole Augustus after the demise of Licinius in 324. There is an inscription of this type on the Arch of Galerius in Thessaloni$\mathrm{ke} ;{ }^{33}$ it is a little strange, however, that the images of Diocletian, Galerius and their entourage performing sacrifice, along with images of the gods and their temples on the same arch, were not defaced. ${ }^{34}$ It is quite probable that local officials, being mindful of the great achievements of the senior Tetrarchs and having benefited from their patronage, were not always eager to carry out Constantine's directives.

\section{Honouring Emperors: Continuity of Cult after A.D. 305}

The question naturally arises as to what attitudes governed the policy of Constantine, his colleagues in the Second Tetrarchy and their successors (ca. 305-312). ${ }^{35}$ The surviving epigraphic evidence indicates that most of the fourth-century emperors from Diocletian to Theodosius the Great were deified at the time of their deaths, the crucial exceptions being the three sons of Constantine by Fausta who reigned between September 337 to November 361 and Valens, who ruled only the East. $^{36}$ The evidence lies mainly in the Latin epigraphy and implies, on the basis of negative evidence from the eastern provinces, that the custom of deifying good emperors was more carefully observed in the Senate at Old Rome than in Con-

32 ILS 631-633, 636, 637 = CIL VIII 2345-2346; V 8016 and VIII 608 respectively.

33 ILS 634.

34 ELSNER 1998, 87. The sacrifice is located on the south-eastern side of the south-west pillar. MACARONAS 1970, 47-48, pl. 25.

35 On this period, see CALDERONE 1973.

36 BONAMENTE 1988, 135-136. 
stantinople. The period after the death of Constantius II was marked by a broad tolerance of the time-honoured Roman religious traditions. ${ }^{37}$ It is therefore not surprising that Julian the Apostate and the Christian emperors Jovian, Valentinian I, Gratian and Theodosius the Great should have been honoured with deification. Furthermore, the custom of apotheosizing the fathers of emperors who had not held imperial power became something approaching standard practice, along the same lines as Philip the Arab had followed for his father Marinus. One father of a a Christian emperor certainly deified was Theodosius the Elder; ${ }^{38}$ it is likely that Gratian the Elder, father of Valentinian I and Valens, also received this distinction, to judge from the phrasing of a badly damaged inscription found at Cirta in Numidia: ${ }^{39}$

To a man of most fortunate remembrance and one who will be celebrated through all the ages, Gratian, the father of our lords and principes Valentinian and Valens, noble and always triumphant Augusti. Antonius Dracontius vir clarissimus, acting in place of the Praetorian Prefect of the African provinces, dedicated (his) statue adjacent to the Capitol, under the supervision of Valerius [...] equestrian (vir egregius) priest of the imperial cult (sacerdotalis).

This inscription provides important clarification as to how and where the images of deified emperors were emplaced. In this instance it was set up adjacent to the Capitol (iuxta C[apitolium]), the temple of every Roman colonia that housed the triad of Juppiter, Juno and Minerva. The provincial priest of the cult carried out the installation. The co-emperors' deified father may well have been the object of honorific rituals at the hands of people involved in sacrifice in the Capitol. Gratian's consecratio took place not long after the accession of Valentinian I and Valens to imperial power, for Antonius Dracontius is known to have been active ca. $365 .^{40}$ It was a natural act of gratitude to a father whose services had paved the way for the brothers' imperial investiture. The "divine character" of the elder Gratian is explained in Ammianus Marcellinus' brief laudatory biography. ${ }^{41}$ The deification of Valentinian I proceeded without any objection and is recorded in an inscription found at Gigithi in Byzancena (ca. 378): ${ }^{42}$

For Valentinian (II), the most vigourous princeps Augustus, begotten from a divine lineage. F[1(avius)] Vivius Benedictus, [equestrian] governor of the province of Tripolitania, dedicated (this) to his numen and majesty always.

Where deification was not possible, the panegyrists took over the task, following the example of the anonymous panegyrist who alluded, while writing in A.D. 291,

37 TROMBLEY 1993-94, 1:62, 110 n. 46.

38 ILS 1277, 8950.

39 ILS $758=$ CIL VIII 7014.

40 Cod. Theod. 10.1.5 and 11.1.11.

41 Amm. 30.7.2 (quotations are from the Loeb Classical Library translation, ROLFE 1935-40).

$42 I L S 779=C I L$ VIII 10489. E. Diehl omits the inscription from ILCV, evidently reckoning that the dedication of this type could not have been made by a Christian. Cf. "Fl. Vivius Benedictus 4" in PLRE 1:161. 
the divine parentage of Maximianus Herculeus (siquidem vos dis esse genitos ...). ${ }^{43}$

The starting point for this discussion must inevitably be the death of Fl. Valerius Constantius, Herculean Caesar in 293-305 and Augustus in the West 305-6, and the father of Constantine. Constantius I was the first of the college of Tetrarchs to die and become divus. As has been seen, there is no correlation between the religious opinions of particular emperors and their deification. We might take Constantius I as an example. There is little evidence to verify or circumscribe his religious beliefs, apart from cultic inscriptions erected in his name. They bear little reference to any sense of personal religion. It was left to Eusebius to pass on the story that on his deathbed in Eburacum Constantius "offered a prayer of thanksgiving to God" for the arrival of his eldest son Constantine, set his affairs in order and then expired (25 July 306$).{ }^{44}$ The terminology of divine names during this period is unusual. There was a strong tendency to designate the god of one's own devotion using terms such as 'the divine', 'the divinity' and the 'highest divinity'. It was a type of theological correctness that was largely confined to the late Tetrarchic period. Eusebius' description is consistent with this transitory phase of Mediterranean religious history. Constantius is elsewhere described as being "on friendly terms with the God over all" and "attached to what pleases God"; it is also said that he recognised "only the God over all" and condemned "the polytheism of the godless". ${ }^{45}$ All this was fairly easy to come by, and could have meant practically anything in the monotheistic jargon of the late Tetrarchic period, assuming that Eusebius is expressing the actual terminology that Constantine the Great reported to him about his father's beliefs - something that may be open to question. ${ }^{46}$

Eusebius' claim that Constantius was the first of the Tetrarchs to be deified is corroborated by the epigraphic evidence - not least because his predecessors the Augusti Diocletian and Maximian had retired and were going to be alive for many years: "He was the first of the four to be proclaimed one of the gods by the [Romans], being deemed worthy of every honour that could be bestowed on an

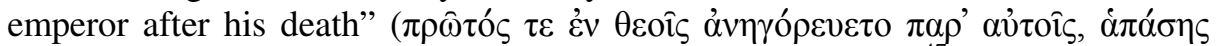

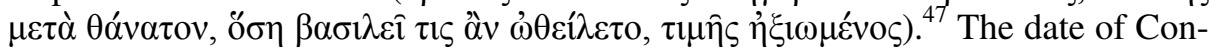
stantius' deification was quite early. An inscription found near Chester in Anglia seems to put this well before Caesar Constantine's march on Rome in 312. It is unknown when, or if, the Senate in Rome ratified this: ${ }^{48}$

43 Paneg. XI (III) 2.4. See RodGERS 1986, 77-78.

44 Eus. v. Const. 1.21 (CAMERON - HALl 1999, 76).

45 Eus. v. Const. 1.13.1, 1.14.1 and 1.17.2 (CAMERON - HALL 1999, 74, 76).

46 It is probably better to disregard Eusebius' claim that Constantius surrounded himself with Christian holy men at this juncture; however, it cannot be excluded that he consulted with the bishops of the larger cities lying inside his jurisdiction.

47 Eus. h. e. 8.13 .

48 ILS 682. Dessau was convinced that the inscription belonged to a time near the army's proclamation of Constantine as Augustus. The deification would have had to be formally voted by the 
To emperor Caesar Flavius Valerius Constantinus, pious, fortunate nobilissimus Caesar, son of the deified (divus) Constantius, pious Augustus.

Constantius' deification was not the last; there is good evidence to show that the emperors of the First and Second Tetrarchies were all formally deified except Maximinus Daia, who had to be condemned for reversing Galerius' edict of toleration for the Christians. Maxentius' son Romulus became divus after his death sometime after his second consulship 309, as an inscription from the Circus of Maxentius indicates: ${ }^{49}$

To the deified Romulus, a man of most noble remembrance, consul ordinarius for the second time, son of our lord Maxentius unconquered and perpetual Augustus, grandson of the deified elder Maximian and of the deified younger Maximian and [...].

Furthermore, an inscription at Caesarea in Mauretania reports: ${ }^{50}$

To the son of the deified Maximianus, son-in-law of the deified Maximianus (Galerius), most fortunate emperors, our lord M. Aurelius Valerius Maxentius, perpetual emperor of the whole world, pious, fortunate, unconquered and most glorious perpetual Augustus. Valerius Faustus vir perfectissimus, governor of the province of Mauretania Caesariensis, dedicated (this) to his guardian spirit (numen) and majesty.

The two inscriptions were cut between early May 311 to 28 October 312, after Galerius' death but before Constantine's victory at the Milvian Bridge. ${ }^{51}$ The younger Maximianus mentioned here was in fact Galerius (viz. Maximianus Galerius), the Augustus in Oriens, who died in 311, and who was evidently young Romulus' father-in-law. Maxentius' presence in Rome may have secured the support of the Senate for the three deifications. ${ }^{52}$ Maximian and Galerius also became divi, once again before Constantine took possession of Rome in late 312. It would have been difficult for Constantine or any succeeding Christian emperor to abrogate these arrangements, and it is doubtful that there were any pragmatic reasons for doing so, notwithstanding the biased denunciations of the Tetrarchs found in the works of Christian writers like Eusebius and Lactantius. There were sound reasons for emphasising the dynastic connection, namely to legitimize the succession of Fausta's sons after the execution of Constantine's half-brothers in September 337, for Constantine's three sons by Fausta had the elder Maximian as

Senate and the decree returned to Constantine's imperial residence before it got into the provincial epigraphy.

49 ILS $673=$ CIL VI 1138.

50 ILS 671.

51 BARNES 1981, 47, 206, puts the consecratio of the elder Maximianus ca. 317/18 at the instigation of Constantine. But see his p. 304 n. 126.

52 Maxentius held the consulship four times during his brief reign, in 308 with his son Romulus, in 309 with his son Romulus both for the second time, in 310 alone, and in 312. See ILS 668 . 
their grandfather. ${ }^{53}$ The accuracy of this interpretation is proven by a milestone inscription of Constantius II on a stretch of military highway near Sirmium (present-day Mitrovica) running between the Save and Drave rivers (ca. A.D. 354): ${ }^{54}$

Five miles. Emperor Caesar Flavius Iulius Constantius, pious, fortunate Augustus, greatest victor, eternally triumphant, [son] of the deified Constantine, best and greatest princeps, grandson of the deified Maximianus and Constantius, descendant of the deified Claudius ... with proconsular imperium for the roads that have been strengthened, bridges rebuilt (and) the Republic restored, laid quinarius stones across Illyricum for the distance of 346 miles from Atrantis to the Save river.

That this was the broad consensus during the reign of Constantius II is corroborated in literary documents like Julian's First Encomium dedicated to him and in the epigraphy of the fourth-century.

There is a consistent progression in the claims being made for the Constantinian dynasty, as diachronic analysis reveals. The divine status of Constantius I as the father of Constantine began to be attested in the epigraphy of Italy not later than 314, during C. Caeionius Rufius Volusianus' service as consul ordinarius for the second time and praefectus urbi in Rome, who erected a dedicatory inscription to Constantine in the forum of Trajan: ${ }^{55}$

To our lord, restorer of the human race, enlarger of the Roman empire and its rule, founder of eternal security, Flavius Valerius Constantinus fortunate, Maximus, pious perpetual Augustus, son of the divine Constantius, perpetually and everywhere revered (filio divi Constanti semper et ubique venerabilis). C. Caeionius Rufius Volusianus, vir clarissimus, consul ordinarius, praefectus urbi, acting as judge in sacred affairs, who consecrated it to his guardian spirit (numen) and majesty.

Constantine was not averse to his genius or guardian spirit being saluted at a time when the memory of Christian martyrs who had died refusing to pour libations in imperial cult was still vividly alive. The phenomenon is apparent in Italy even earlier, on a milestone in the territory of Harpinium dated to 313, but in the latter case the grandiloquent phrases are lacking and Constantine is designated merely as imperator Caesar with the patronymic. ${ }^{56} \mathrm{He}$ is saluted in an inscription at Vicetia in 328 as "best of princes in human affairs, son of the deified Constantius, born for the good of the Republic" (humanarum rerum optimus princeps, divi Constanti filius, bono reipublicae natus), a clear intensification of the familial rhetoric that qualifies the obscure origin of Constantine's mother Helena in a positive way. ${ }^{57}$

53 Fausta herself suffered damnatio memoriae, with the erasure of her name and that of Caesar Crispus (inter 8 November 323 - fin. 326). ILS 710.

54 ILS $732=$ CIL III 3705. See also ILS 730 = CIL II 4844 (prope Bracam in Callaecia).

55 ILS $692=$ CIL VI 1140 .

56 ILS 693. The inscription is dated by Constantine's third consulship.

$57 \quad I L S 697=C I L \mathrm{~V} 8011$. 
The commemoration of Constantine with his deified father is more typical of the provinces on the northern coast of the Mediterranean than the rest of the empire. It is commemorated, for example, on a milestone at Cabassa in Narbonensis, on which the elder Maximian is mentioned as his nepos, an apparent reference to his status as father-in-law of Constantine after the latter's marriage to Fausta. ${ }^{58}$ Such acknowledgements are lacking in any number of other inscriptions dedicated to his numen, including at Ain-Roua in Mauretania, Cirta, Luxor in Egypt, Ostia, Sitifis in Mauretania and Uci Maioris in Africa. ${ }^{59}$ There is an undated inscription at Troesmis in Scythia Minor, where he is designated as a "son of the Augusti" (filius Augustorum). As Hermann Dessau explains, this inscription was erected in the Praefecture of Thrace, which lay under Galerius' jurisdiction; it may well reflect eastern practice, inasmuch as Lactantius uses the same phrase. Maxentius and possibly Maximinus Daia were also known by this title. ${ }^{60}$ The question remains as to whether Constantine's particular status, as the first emperor of the Tetrarchy to have a deified father, was being suppressed in Oriens in the interest of collegial politics and of putting Constantine's ambitious nature into the shadows.

Eusebius of Caesarea was the first Christian literary figure to propagate the story of Constantine's descent from his purported ancestor, the emperor Claudius II Gothicus. It was not original to him. Dessau regards this as having begun not long after the death of the elder Maximian in $310 .^{61}$ This particular facet is attested in an undated inscription dedicated by the superintendent of an imperial weapons factory (praepositus fabricae [armorum]): ${ }^{62}$

To the enlarger of the Roman empire, founder of public peace, our lord Flavius

Constantinus Maximus, victor, perpetual Augustus, grandson of the deified

Claudius, son of the deified Constantius. Sertorius Silanus vir perfectissimus, su-

perintendent of the factory, dedicated it to his guardian spirit and majesty.

The off-hand way in which the equestrian officer delivered the message of Constantine's descent from Claudius (divi Claudi nepos) suggests that it had been in wide circulation for some time. It also appears in an undated inscription at Rome commemorating the reconstruction of the aqueduct of Virgo in Rome, along with the standard dedicatory formula of the imperial cult (devotus numini maiestatique eius). ${ }^{63}$

The well-known inscription at Hispellum, in which the college of emperors Constantine and his three sons by Fausta - permitted the foundation of a temple (aedes) and local priesthood of the cult of the gens Flavia at the request of the

$58 \quad I L S 684=C I L$ XII 5470.

59 ILS 687-688, 691, 696.

60 Lact. mort. pers. 32.

61 BARNES 1981, 34-36, 40-41 etc.

62 ILS 699 = CIL XI 9. See DESSAU 1889, 342.

63 ILS 702. 
curiales, has been frequently commented upon. ${ }^{64}$ As a token of the progress of the imperial cult, the inscription is significant in two respects: it points to the continued existence of priests of the imperial cult (here designated as sacerdotes), and the proscription of sacrifice in the name of the dynasty. ${ }^{65}$ The latter was the culmination of a long process. The decree is far from being earth-shaking. It permits the full range of spectacles associated with non-Christian religious festivals, a principle that remained in force throughout the fourth century. ${ }^{66}$ Sacrifice is proscribed merely in the cult of the gens Flavia at this temenos: "lest the building dedicated to our name be polluted by the errors of contagious religious excess" (ne aedis nostro nomini dedicata cuiusquam contagiose superstitionis fraudibus polluantur). The word contagiosus is confined in the secular literature to a certain Vegetius' De arte veterinaria sive de mulomedicina, and tends to appear in the terminological contexts of medical science, in phrases such as passio contagiosa and scabies contagiosa (ca. 420). The Hispellum inscription clearly uses the term in the sense of spiritual disease, with possible physical repercussions, as expressed for example in Book 2 of Porphyrius of Tyre's work on abstinence from animal meat, where sacrifice is denounced because, in Pythagorean theology, it was thought to attract the daemons of disease. ${ }^{67}$ The cult of the gens Flavia continues to be attested in the epigraphy thereafter through the pontifex Flavialis in Rome. ${ }^{68}$ It is possible that the ritual aspect of the cult of deified emperors was being exaggerated in some quarters. A useful corrective to this comes from the pen of Vegetius in his De re militari where he emphasises service to the monarch as the proper attitude: ${ }^{69}$

When an emperor has accepted the name of Augustus, loyal devotion must be offered and ceaseless service must be applied to him, as though to a present and incarnate God. For either in peace or in war one serves God when one loyally loves him who rules under God's authority.

\section{Temples}

Shrines of the deified emperors existed in most urban settlements and permanent military camps between A.D. $244-395 .^{70}$ There are many instances of this phenomenon, as for example the temple at Durostorum in Moesia Inferior mentioned in the martyrdom narrative of St. Dasius, where there were 'images' of the living

64 ILS 705. GASCOU 1967; CURRAN 1996.

65 On sacrifice in the imperial cult before 305, see PRICE 1984, 207-233.

66 For example, Cod. Theod. 16.10.8.

67 Porph. abstin. 2.35, 40, 42-43, ed. NAUCK 1860.

68 CIL VI 1690, 1691, 1694.

69 [*Veget. mil. 2.5], quoted from COLEMAN-NORTON 1966, 302.

70 For a list of the temples of the imperial cult in Asia Minor, see Price 1984, 249-274. For central and southern Greece: TRUMMER 1980. 
emperors of the First Tetrarchy. ${ }^{71}$ At times the imperial images were housed in the temples of other gods. ${ }^{72}$

The era of the Christian empire saw the destruction of many pagan temples, and the conversion of many others into Christian buildings. Friedrich Deichmann, writing in 1939, came up with a provisional list of temples converted into Christian churches. ${ }^{73}$ Subsequent research in epigraphic and hagiographic texts indicates the execution of many more temple conversions than Deichmann was able to trace. ${ }^{74}$ As R.P.C. Hanson has suggested, the main sequence of converting temples into churches took place from the late fourth century onward. ${ }^{75}$ It is significant that among the recorded examples there is not a single instance of a temple specifically dedicated to the imperial cult being converted for other uses, whether secular or ecclesiastical. It has been argued that one of the excavated temples in Eretria on the island of Euboea housed many images dedicated to the imperial cult. ${ }^{76}$ Its date of destruction must be near that of the Parthenon and Asklepieion in Athens, which was probably ca. $484 .{ }^{77}$ An examination of the epigraphic and historical evidence suggests that, although some emperors like Maximianus Galerius, Diocletian's original junior colleague in the Iovian branch of the Tetrarchy, were subjected to damnatio memoriae in some local contexts, there does not always seem to have been a systematic attempt to remove their statuary, altars and other trappings of cult when it was a case of harming buildings and shrines dedicated to the imperial cult proper. Actions of this kind seem not to have been tolerated, particularly if the actors were not agents of the imperial administration, such as bishops, provincial curiae and city councils. ${ }^{78}$ It must be recognised that shrines for deified emperors did at times exist in temples dedicated to other great gods. This may partly explain why some temples were left intact in the interest of civic functions. In instances where a building was being converted or destroyed, it would have been necessary to remove the imperial images and install them at another appropriately consecrated installation before the contemplated action could take place. Certain of these features will become obvious through a survey of the epigraphy.

Turning to the inscriptions, the imperial cult is indicated in many different ways, sometimes indirectly. The most common form of expression is a prayer, sacrifice or construction of a small shrine on behalf of the ruling prince or coemperors. The offering was often made to a tutelary spirit on behalf of the emperor, as for example his Victoria or Nikē, Fortuna or Tychē, or else on behalf of

71 M. Das. 7 (MusuriLlo 1972, 276-277).

72 IGR I 1181.

73 DEICHMANN 1939, 105-136.

74 BAYLISS 2004, 121-129; SPIESER 1976.

75 HANSON 1978.

76 The excavator (SCHMID 2001, 140-141) does not commit himself to a particular date.

77 TROMBLEY 1993-94, 1:310-311. MACMuLLEN (1997, 187-188 n. 59) provides no evidence (or arguments) for his supposition that these events took place some decades earlier.

78 On the city councils and priesthoods in the fourth century, see HORSTKOTTE 1989. 
his Salus or Hygeia. These sacred acts were sometimes performed under the immediate impact of events, particularly in wars and in case of regime change. One such inscription commemorates a military victory by the $d u x$ of Noricum and Pannonia Superior. The date was 27 June $310:^{79}$

A sacred offering (sacrum) to the Victoria Augusta on behalf of the safety of our lords Maximinus and Constantine and Licinius perpetual Augusti. Aur(elius) Senecio vir perfectissimus (and) dux ordered that a temple (templum) be made anew for her (or 'their') numen because of a vow, through the instance of Val(erius) Sambarra, praepositus of the Equites Dalmatae Aquesianes Comitatenses, because of the victory that took place on the fifth day before the Kalends of July when Andronicus and Probus were consuls.

This engagement is otherwise unattested. Shrines of this type existed in many places and were being constantly set up in localities where Roman military forces achieved local successes against the barbarians. Armies passing on campaign would pause to perform the necessary rituals at these sites, even in the later fourth century. A good example of this was seen during Julian Augustus' campaign in Mesopotamia in 363. Ammianus Marcellinus reports: ${ }^{80}$

Setting out from [Circesium] we came to a place called Zaitha, which means 'olive tree'. Here we saw, conspicuous from afar, the tomb (tumulum) of the emperor Gordianus. ... When Julian had there, in accordance with his native piety (ingenita pietas), made offerings to the deified emperor (consecratus princeps), and was on his way to Dura (a deserted town), he saw a troop of soldiers in the distance and halted.

The monument does not seem to have survived to the present day, so its configuration cannot be deduced. One might suppose from this that the customary rules of ritual purity did not apply in connection with sacrifices performed at the tombs or cenotaphs of deified emperors, even though their corpses might have been interred within.

Other open-air shrines may have existed, although the sources do not always provide the requisite detail. For example, Lactantius mentions an open-air site outside Nicomedia in Asia Minor, one of the eastern imperial residences before the foundation of Constantinople. The place was used on 1 May 305 for the ceremonial abdication of Diocletian: ${ }^{81}$

The gaze of all was upon Constantine ... the soldiers who were present, the military officers who had been chosen and summoned from the legions ... were making their prayers for him (vota faciebant). There was some high ground just under three miles out of the city, on whose summit Maximian had himself assumed the purple, and on which a statue had been put up with a statue of Jupiter (et ibi columna fuerat erecta cum Iovis signo).

79 ILS 664.

80 Amm. 23.5.7-8.

81 Lact. mort. pers. 19.2 . 
The image of Jupiter certainly commemorated the Iovian branch of the Tetrarchy. The question remains whether an altar existed for votive offerings and whether there was a synoikism with the cult of the father of the gods with that of the $n u$ mina of the emperors. The latter seems quite possible. This practice was known particularly in Egypt. A rare dedication from the brief reign of the usurper Quietus of A.D. 260 found at Koptos in Egypt seems to indicate such a synoikism. The inscription is broken in many places:

On behalf of the security (diamonē) of Quietus, one of the Augusti, to the gods [housed in the same temple], the temple (temenos) and statue in it [---] Areios whose family name is [---] city councillor [---] of the Red Sea with [---].

The statue in question was evidently that of the emperor Quietus, since there appears to be no space for names of the other divinities on the stone. There is a possible synoikism of a shrine of the emperors with a god at Gerasa. The inscription concerns a stoa dedicated to Artemis, whose effigy appears on locally minted coins: ${ }^{83}$

For a blessing. In the year 142 (of the province of Arabia). On behalf of the preservation of the Augusti. Using their own funds the men who reverence the gods (or 'the emperors') made the stoa for Artemis and the cistern in the year 142 (= A.D. 247/8).

The dedicants may have been the local college of priests of the imperial cult. They are styled as 'the reverers' (sebomenoi), a term that is normally devoid of any technical meaning, but here it could be a play on the Sebastoi or Augusti named at the start of the inscription. It is not unlikely that the stoa contained altars dedicated to both Artemis and the deified emperors, but positive proof is lacking. The reigning emperors were once again Philip the Arab and his son.

It is also likely that statues of reigning emperors were housed in the ancillary shrines of temples that were dedicated to local and ethnic divinities. This must have been particularly the case in the Provincia Arabia during the imperium of Philip the Arab, who established in his native town Philippopolis the cult of his father, the deified Marinus, who goes by the name of "Marcus Iulius Marinus

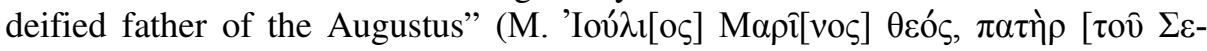
$\beta \alpha \sigma \tau o v]]$ ) in one inscription, in the instance a statue erected by the ducenarius Aurelius Antoninus. ${ }^{84}$ The legate of the province of Syria ( $\left.\dot{v} \pi[\alpha \tau 1 \kappa o ́ c]\right)$ was also

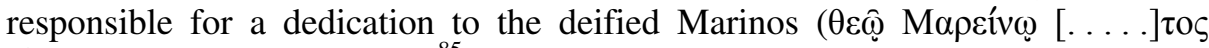
$(\dot{v} \pi[\alpha \tau \iota \kappa o ́ s])$ at Philippopolis. ${ }^{85}$ The terminus ante quem of these inscriptions was the overthrow and apparent damnatio memoriae of Philip in 249. The deification of the fathers of emperors who had not themselves held the imperial office became a tendency from this time onwards, among them Gratian the Elder, father of

82 IGR I 1181.

83 IGR III 1363.

84 IGR III $1199=$ [*Waddington 2075]. On Marinus, see KÖRNER 2002, 49-54.

85 IGR III 1200a. 
Valentinian I (364-375) and the elder Theodosius, father of Theodosius the Great (379-395), as noted above.

Prayers for the safety ( $\left.\sigma \omega \tau \eta \rho^{\prime} \alpha\right)$ of the Arab emperor abound elsewhere in Arabia. An apposite example is seen in an inscription of 15 October 245 at Admedera: ${ }^{86}$

For the preservation of our lords the emperors, the Caesars Marcus Iulius Philippus (senior and junior) the Augusti, the temple of Aeichala was finished and dedicated in the time of the sacred treasurers M. Aurelius Aneus son of Gaōrus

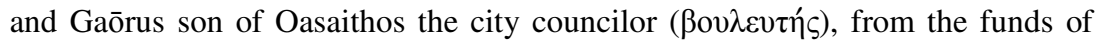
the god.

Aeichala, a seldom-attested Arabian divinity with an established local cult, was invoked as though he were Philip's tutelary god. The emperor's name and title were erased in consequence of his ostensible damnatio memoriae after Decius assumed the imperium. Another inscription at Admedera, whose date needs to be emended either to A.D. $294 / 5$ or $394 / 5$, reflects continuity in the imperial cult among military officers down to the time either of the Tetrarchy or the Theodosian dynasty: ${ }^{87}$

For the preservation of (our) lords [the Augusti?], Annianus, groom (stratōr) of the commander of the Ala Vocontiorum, built the architrave (to epistylion) from his own funds in accordance with his own vow and that of his children.

The architrave was emplaced in a temple of the deified emperors to judge from the prayer at the beginning. There are other less specific inscriptions of this character. ${ }^{88}$ The size and impressiveness of these installations varied considerably, and they were as likely to appear in the countryside as much as in the cities. So for example at Meron in Phrygia Salutaris, a badly damaged inscription with some doubtful readings, probably of the reign of Gallienus (A.D. 260), reports that the revenue collector ( $\mu 1 \sigma \theta \omega \tau \eta \dot{s}$ ) of the nearby imperial estates set up an installation: ${ }^{89}$

[On behalf of the perpetual safety emperor Gallienus - Ger]manicu[s Caesar and] of the dē [mos of Prymnessos and] the démos of Nac[ol]ia. Appa the revenue collector [of the villages] of Caesar with his [wife Rho]do offered the building of [the temple?] from his own funds. [---] son of Alexander (the) village headman

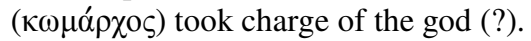

Prayers for the preservation of the emperor were an important pro forma in inscriptions mentioning the construction of installations designed for the protection of the empire. Inscriptions use the language of sanctification as though such sites were altars or other cultic appurtenances, as with a tower erected at Adraae in

86 IGR III $1093=$ [Waddington 2562g].

87 IGR III 1094

88 IGR IV 1487 (Sedikeui, Asia, A.D. 251-253); IGR IV 568 (Aezani, Asia, reign of Diocletian).

89 IGR IV 592, 593. ILS 540. 
Arabia: "For the preservation of our lord the emperor Gallienus Augustus, the

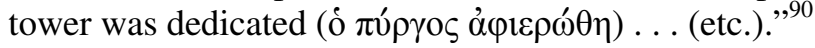

A reigning emperor might sometimes be characterised as a divinity in the conventionalised language of a local cult. This was the case at Baitokaikai in Phoenice, where the co-emperors Valerian and Gallienus renewed the privileges of the priesthood who served the local Zeus-Baal that had originally been conferred by one of the Seleucid kings sometime between 280 and 264 B.C. (regum antiqua be[n]eficia).$^{91}$ The resolution of thanks voted to the co-emperors by the

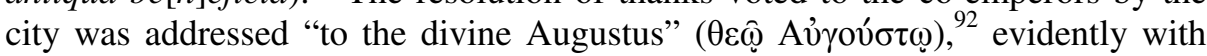
the senior emperor Valerian in mind.

At other times the emperors are merely mentioned in the dating formula, where the imperial cult stands in niggardly contrast to the recognised powers of great gods and the spirit of devotion found in their adherents. One thinks for example of an inscription from the time of the Tetrarchy at Akoris in Egypt in which the priest Dydymos (sic) commemorates the life-giving strength of the Egyptian gods (August 290), and a prayer of thanksgiving after return from exile dedicated to the "highest divinity who hearkens" by a certain Aurelius Valerius Sogous son of Olympus at Panticapaeum in the Crimea (A.D. 306). ${ }^{93}$ There is also much terminology that expresses an empty formalism, as for example the "most

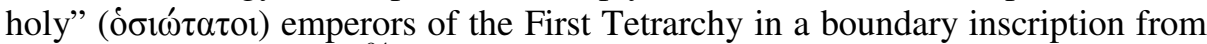
Doliche in Euphratensis. ${ }^{94}$

Temples of the deified emperors might remain standing for centuries, as for example the sacred Kaisareion in Smyrna. ${ }^{95}$ A striking example is seen in the temple of the deified Vespasian at Baiae near Naples, which was still in use for public functions in A.D. $289 .^{96}$ Another example of this is the temple of Hadrian at Ephesus. It was repaired after an earthquake, possibly the one of A.D. 358, and a new frieze, making use of pre-existing material, was incorporated into its interior. Probably dating from the time of Julian, Section D of the frieze may represent himself (a man of average height in military garb), his father (a somewhat taller man wearing a mantle that resembles a toga) and his grandfather the deified Constantius I. ${ }^{97}$ It is one of the chronologically latest examples of pre-Christian religious art of any kind to have survived.

90 IGR III 1286 (A.D. 261/2).

91 It is unknown which Antiochus was the author of the grant.

92 IGR III $1020=$ CIL III 184.

93 IGR [*I-II] 1134; IGR III 873.

94 IGR III 1002.

95 IGR IV 1480.

96 ILS 4175.

97 FLEISCHER 1967. The author of this study does not commit himself absolutely to the identifications I have made. The figures in question are D8, D9 and D12, pp. 31-34 and pl. 17. It is suggestive that the deified figure I take to be divus Constantius stands next to a figure that seems to be Hercules, the tutelary divinity of Constantius' branch of the Tetrarchy. It is also thoughtprovoking that the figure I take to be Julian (D12) - who distinguished himself as a military 


\section{Images, Priesthoods, Sacrifice and the Accessories of Cult}

There is a vast number of inscriptions between ca. 244 and 395 recording the erection of images of the emperors. ${ }^{98}$ In most cases it is impossible directly to establish these images' character as accessories to the imperial cult. The stones on which the dedications are made have frequently been moved from their original locations for secondary use as building materials. It is difficult to determine whether the images were originally sited in temples of the imperial cult or simply positioned in urban public spaces as tokens of honour for reigning emperors; it is not always clear in what kind of building or shrine they were housed. ${ }^{99}$ When cultic personnel and the activities they sponsored are mentioned, however, it is possible to draw such connections. This is borne out in an inscription possibly from the reign of Probus at Nikopolis in Moesia Inferior. The priesthood expresses its views in Greek, but there must have been a strong Latin ethnic element in the local population: ${ }^{100}$

The council and most sacred demos of the city of the Nicopolitans on the Danube erected the (image of) M. Aurelius Probus, master of land and sea, fortunate Augustus, [G]e[t]icus (?) Parthicus Maximus with blessings, in the consulship of $\mathrm{Cl}$. Annius Natalianus, legatus propraetore (antistrateggos), under the supervision of Asklepiodoros son of Asklepiades, sacerdotalis of the province (archieratikos).

Asklepiades was the priest of the imperial cult in Moesia Interior and may well have supervised the emplacement of this image of a living emperor in the local sebasteion. ${ }^{101}$ The examples that follow belong mostly to cultic contexts or make reference to cultic behaviour. It must be borne in mind that the images of deified emperors appear to have held the same status as those of recognised great gods.

Reports about imperial images in historical sources are often vague as to the context. A good example of this is found in Ammianus Marcellinus' report about the return of Jovian to Antioch after the retreat from Mesopotamia in 363. I must quote our eyewitness at length because of its theological implications: ${ }^{102}$

[W] came by long marches to Antioch; where for successive days, as though the divinity were angered, many fearful portents were seen. . . . For the statue of Caesar Maximinus, which stood in the vestibule of the imperial palace, suddenly dropped the brazen ball, in the form of the globe of heaven, which it was holding.

commander in Gaul - is surrounded by the female divinities Athena, Aphrodite and Hekate. Julian may have held Athena in special reverence as the philosopher goddess, and Hekate not least because of the influence on him of the theurgist Maximus of Ephesus. These opinions are provisional, pending further study of the cultural, political and religious background.

98 For the period under consideration here, see PRICE 1984, 175-177, 203-206.

99 These observations are a product of reviewing the inscriptions collected in Cagnat's IGR.

100 IGR [*I-II] 582.

101 Other inscriptions use a similar formula, but fail to mention cultic personnel, e.g. IGR IV 626 (Trajanopolis, Asia, A.D. 251/2).

102 Amm. 25.10.1-2. 
It is at first sight surprising that the image of Maximianus Herculeus, Diocletian's co-Augustus in the Tetrarchy, was still found in the imperial residence at Antioch in view of the possible damnatio memoriae of his former senior colleague in 311, as reported by Lactantius. ${ }^{103}$ Perhaps the measure was confined to the West, with Licinius unwilling to act on it in the East and the matter becoming otiose after the latter's demise in 323. It is again possible that the image in question stood in a shrine, in view of its presence in the palace.

The temples of the imperial cult were still quite active in the fourth century. There is the example of a temple at Capua in the Campania, where a certain Romanus Iunior, a priest of the imperial cult of the province (sacerdos ferialis domnorum), was ordered to carry out an annual calendar of festivals, including the vows (vota) for the preservation of the emperors, at the command of the coemperors (viz. Theodosius I, Valentinian II and Arcadius). These he performed on 22 November A.D. 387, apparently the birthday of Valentinian II. ${ }^{104}$ It seems that there was no objection to these activities or the other local festivals in the calendar, as long as the proscribed sacrifices were avoided.

Sacrifices normally accompanied the celebration of the cult of the deified emperors. ${ }^{105}$ Clear examples of the ritual are nevertheless difficult to find in the sources, apart from the Christian martyrdom narratives. Maximinus Herculeus is known to have performed sacrifices. It is reported that, after his installation ceremony for the consulship was interrupted by a barbarian raid, he celebrated the victory in Trier with sacrifices. ${ }^{106}$ The panegyrist characterizes the event as a sacrifice to the emperor's own numen (sacrific $[i]$ s . . . numini tuo implesti). Sacrificial liturgies are exceptionally difficult to trace under the fourth-century Christian emperors, who surely followed the example of Constantine as expressed in the Hispellum inscription in abhorring those proceedings. Yet the use of incense in front of imperial statues and portraits continued even among Christians, to judge from the remarks of Philostorgius the ecclesiastical historian. ${ }^{107}$ Whether sacrifices continued thereafter in the imperial cult is a worthwhile question. There would have been little interference with this during the period of toleration between A.D. 361 and 381. Similarly, there may have been a liberalisation in some localities during the administration of the Praetorian Prefect Flavius Eutolmius

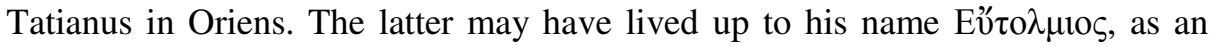
inscription from Antinoopolis in Egypt suggests. It was carved on an altar dedicated to the co-emperors: ${ }^{08}$

103 Lact. mort. pers. 42.1: Eodemque tempore senis Maximiani statuae Constantini Constantini iussu revellebantur et imagines ubicumque pictus esset, detrahebantur. Cf. DOWNEY 1953, esp. $114 \mathrm{n} .1$.

$104 I L S 4918=$ CIL X 3792.

105 PRICE 1980.

106 Paneg. X (II) 10.6.5. RodGers 1986, 75-76.

107 See next section.

108 OGIS 723. Cf. WACHSMUTH 1873. 
Flavius Eutolmius Tatianus, the most distinguished Praetorian Prefect, consecrated (this altar) with the customary dedication, in the time of the distinguished governor Flavius Septimius Eutropius, on behalf of the emperors of (all) the earth under the sun, our triumphant masters, Valentinian (II), Theodosius (I) (and) Arcadius, the eternal Augusti, and the most distinguished Flavius Honorius.

This altar is a final reminder of the sacrificial tradition of the imperial cult. Local devotees may have placed incense on the altar fires, just as some Christians did beneath the porphyry statue of Constantine in Constantinople. ${ }^{109}$ The survival of this unique document is suggestive that the altar in Antinoopolis remained in long use, perhaps for the duration of the Theodosian house in Oriens. ${ }^{110}$

The cultic personnel of the temples have been thoroughly documented. ${ }^{111}$ There is therefore little more to do than note some particularly striking cases. The offices of provincial high priest of the imperial cult (sacerdotalis provinciae) and municipal priest (flamen perpetuus) are attested in many places from the beginning of the period under consideration here until A.D. 525/6. The preponderance of epigraphic evidence comes from the African provinces. ${ }^{112}$ It is clear that, in addition to the priestly functions, the sacerdotales and flamines had important civic duties to perform, like a certain Ecidicius at Cirta-Constantina who took charge of repairing the principal water conduit bringing water into the city. ${ }^{113}$ This fact in no way vitiated their role as curators of the imperial images and shrines. Some municipalities had large numbers of flamines. For example, an inscription at Thamugadi in Numidia dating from the later years of Constantius II or Julian provides a register of men (album ordinis co(loniae) Tham(ugadorum)) who had recently held key municipal offices. Among them are two sacerdotales, with a flamen in the prescript. ${ }^{114}$ There is a second inscription from the reign of Julian where the sacerdotales are designated as coronati provinciae. ${ }^{115}$ The imperial cult flourished at this time. The flamen Flavius Aquilinus supervised the erection of an altar dedicated to this emperor. ${ }^{116}$ It is likely that the priesthoods retained certain cultic functions until the early sixth century. It is therefore difficult to understand why, with the province of Byzacena under the administration of the Vandal kings, a man would identify himself as a "Christian flamen" (fl(amen) $p\left(\right.$ er)p(etuus) Cristianus (sic)) in a funerary inscription. ${ }^{117}$ It is found in one of the five early Christian basilicas in Ammaedara (A.D. 525/6):

109 See next section.

110 On Egypt, see FrANKFURTER 1998, 265-284.

111 KORNEMANN 1902, esp. 136-142.

112 For the epigraphic record on imperial cult in the towns of Africa, see LEPELLEY 1979, 362-369.

113 CIL VIII 7034.

114 CIL VIII 2403. MOMMSEN 1877, 77-84.

115 CIL VIII 17896.

116 CIL VIII 2387.

117 CIL VIII 10516. Another flamen, identified as a Christian by the usual symbols, is found at CIL VIII 450. 
(Leaf) (Chi-Rho) (Alpha-Omega) (Leaf) Astius Mustelus, a Christian flamen perpetuus, lived seventy-two years. He died (quievit) on the eighth day before the Ides of December in the fourth year of the reign of our lord, king Ildirix.

Christians had never been forbidden to hold office in the imperial cult, but only from taking part in the production of games and theatrical events, as well as participating in sacrifice, as we learn from the Canons 2 and 3 of the Council of Elvira (ca. 305). ${ }^{118}$ It is likely that the priests of the imperial cult mentioned in many public, as opposed to funerary inscriptions, were in fact Christians, but positive proof of cult is invariably lacking.

There is limited evidence for the sponsorship and celebration of games by priests of the imperial cult. For example, we learn of the Augustan games being celebrated in Smyrna ca. 253-259. ${ }^{119}$ More significantly, a fourth-century inscription at Athens mentions the activities of a flamen: ${ }^{120}$

Flavius Septimius Marcelleinus, flamen and ex-agonothete, repaired the gates for

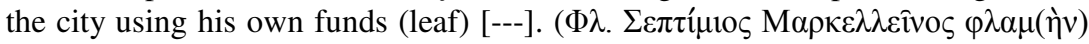

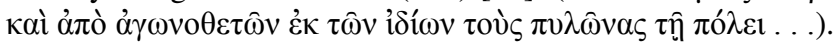

The man embodied many of the functions thus far seen: public works, the ritual aspects of the imperial cult and the sponsorship of games.

\section{Christian Responses}

Christian theological writers had always expressed skepticism about the imperial cult. Their arguments were at times delivered with a degree of deliberate obtuseness and cynicism that is surprising in light of what they surely knew about the realities of the ritual of consecratio, the deification of good emperors after their deaths, and the subsequent honours and rites accorded to them. ${ }^{21}$ References to

118 For text and analysis, see HEFELE 1907, 221-264.

119 IGR IV 1419.

$120 I G$ [*II.2] 5206 (ed. Kirchner).

121 In the period before A.D. 244, there is considerable evidence in the martyr acta for Christians refusing to honour the cult of the emperors by pouring libations and other forms of sacrifice. The relevant examples can be briefly summarized. The first instance of Christian contact with the imperial cult is seen in a martyrdom narrative about Polycarp, bishop of Smyrna ca. 156/7.

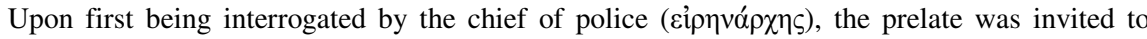
make a simple declaration: "What is wrong with saying 'Caesar is lord' ( forming the sacrifices and attendant rituals and thereby saving yourself?" (M. Polyc. 8, ed. MUSURILlo 1972, 8-9). The next phase of the trial took place in the stadium ( $\sigma \tau \alpha \dot{\delta} \delta 10 v)$ of the city. The proconsul or governor of the province of Asia took charge of the proceedings, asking Polycarp in simple terms: "Have respect for your age. . . Swear by the tyche of the emperor

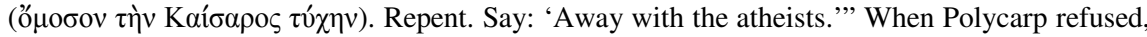
the proconsul posed the question a second time with one addition: "Swear and I will release you. Curse Christ." Polycarp's reply was even less compromising, for it made use of the honorific terminology of the emperors and applied it to Christ: "How can I blaspheme my emperor 
the imperial cult in the martyrdom narratives appear increasingly in the second half of the third century, and particularly in the time of the Tetrarchy. Refusal to swear by the emperor's genius was seldom an issue, and it is likely that Christians felt little repulsion at the rituals connected with the cult. Christians had certainly begun to hold the office of flamen or priest of the imperial cult by the time of the Council of Elvira in Spain in the early fourth century, but probably much earlier, in the last quarter of the third century. ${ }^{122}$ This is consistent with the greater integration of Christians into the wealth and administrative fabric of the late Roman empire.

The references to imperial cult in the martyr passiones require attention, because of the special way in which these documents handle it. The martyr acta are vividly biased against pagan religion, and yet are usually formally respectful toward imperial institutions. ${ }^{123}$ The more historically grounded martyr acta, which make references to imperial cult for the period under consideration here, even if only obliquely, are found in Musurillo's select collection of passiones: ${ }^{124}$

( $\beta \alpha \sigma i \lambda \varepsilon v ́ \varsigma)$ and saviour ( $\sigma \omega \tau \eta \tilde{\rho})$ ?” The latter term appears frequently as an attribute of the Hellenistic kings and Roman emperors, referring to the tasks they performed to relieve the sufferings of the Greek cities in time of famine and barbarian invasion. A third injunction to swear met with a final refusal. It seems plausible that shrines of the emperors existed in many places, and that it was therefore quite a simple thing to conform to the standard ritual. It would become possible in the time of Constantine the Great to envision Christ in Apollonian garb as the divine companion or genius of the Christian emperor, and in later centuries to speak of the tyche of the Christians without denying their monotheistic views. TROMBLEY 1993-94, 2:331, 366. In the early second century this engagement with the language of Greek religion had only just begun. The acta of the Scillitan martyrs who were tried in Carthage on 17 July 180 give a cognate Latin formulation of the principal ideological issues. The proconsul P. Vigellius Saturninus, who personally carried out the interrogation, puts it in these terms, as we have it: "We too are a religious people, and our religion is a simple one. We swear by the genius of our lord the emperor and offer prayers for his safety (iuramus per genium domini nostri imperatoris et pro salute eius supplicamus), which you too ought to do" (M. Scil. 3, ed. MusurILlo 1972, 86-87). The Christians' response to this straightforward formulation was to manipulate the language of the imperial cult to press their own case, as for example: "I acknowledge as my lord the emperor of kings and all the peoples" (cognosco dominum meum imperatorem regum et omnium gentium), where Christ as a divinity usurps the claim of the Roman magistrates to universal empire, and "I pay honour to Caesar as Caesar, but I accord fear to God" (honorem Caesari quasi Caesari; timorem autem Deo; M. Scil. 6, 9, ed. MusuriLlo 1972, 86-89). The latter could be taken as verbal compliance with the demands of the proconsul, apart from the fact that the Christians were refusing explicitly to swear by the emperor's genius. It was all the same a politically correct description of the emperor's ideological posture vis-à-vis the imperial cult, with the deified emperor as "god" and as a living man.

122 JONES 1964, 71.

123 See the discussion of MiLlar 1973; GRADEL 2002, 367-369.

124 MUSURILLO 1972. 


$\begin{array}{llll}\text { Name of Martyr } & \text { Place of Martyrdom } & \text { Date of Martyrdom } & \text { Redaction } \\ \text { Pionius } & \text { Smyrna } & \text { ca. 250/1 ? } & \text { ca. 300 } \\ \begin{array}{l}\text { Fructuosus } \text { et al. } \\ \text { Marinus }\end{array} & \text { Tarragona, Spain } & \text { 21 January 259 } & \text { early 4th cent. } \\ \text { Marcellus } & \text { Caesarea in Palaestina } & \text { ca. 260/1 } & \text { early 4th cent. } \\ \text { or Marcellus } & \text { Leon in Gallaecia } & - & - \\ \text { Julius veteranus } & \text { Durostorum, Moesia Inf. } & \text { Spring 304 } & \text { post 306-308 } \\ \text { Dasius } & \text { Durostorum, Moesia Inf. } & \text { Spring 304 } & \text { late 4th cent.? } \\ \text { Agape, } & & \text { Spring 304 } & - \\ \text { Irene, Chione } & \text { Thessalonike } & \text { 29 April } & \\ \text { Euplus } & \text { Catania, Sicily } & \text { or 12 August 304 } & - \\ \text { Crispina } & \text { Tebessa } & \text { 5 December 304 } & -\end{array}$

It is striking that so many of these texts belong to the period of the First Tetrarchy (284-305), and particularly to the aftermath of the Fourth Edict of JanuaryFebruary 304, which required sacrifice to the gods. Apart from the acta of Dasius, these documents seem to have mostly been written not long after the events, and therefore have a greater chance of reflecting the types of statements made at these tribunals.

References to imperial cult in the pre-Tetrarchic documents are at times no more than passing observations. So, for example, in the martyrion of Pionius, after the latter refuses to sacrifice to the gods, his interrogator responds: "So then

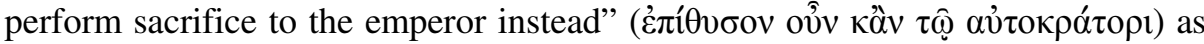
though this was more persuasive and likely to get a positive response from a Christian. ${ }^{125}$ Similarly, in the passio of Bishop Fructuosus, and his deacons Augurius and Eulogus, the proconsul Aemilianus of Hispania Citerior is reputed to have told them: "These [gods] are obeyed, they are feared, they are worshipped. If the gods are not worshiped, then neither are the images of the emperors" (. . nec imperatorum vultus adorantur). ${ }^{126}$ The basis for this prosecution was the failure of Fructuosus and his clergy to comply with the edict of Emperor Valerian (July 258) requiring an empire-wide sacrifice, rather than a specific refusal to honour the emperors. ${ }^{127}$ Once again, it seemed obvious to the magistrate that it was a simple and obvious thing for Christians to worship the emperors, even if they ignored the other cults. The martyrdom of Marinus is rather more explicit. It comes from the pen of Eusebius of Caesarea: ${ }^{128}$

125 M. Pionii 8, ed. MusuriLlo 1972, 146-147. It should be noted that this work shows clear signs of literary re-editing.

126 MUSURILLO 1972, 178-179.

127 The decree called for the immediate punishment of Christian clergy. Cypr. ep. 80.1 (CSEL III 2,839-840 (non vidi latine).

128 M. Marini = Eus. h. e. 7.15, ed. MusuriLlo 1972, 241. 
An army post fell vacant, and according to the order of promotion it was Marinus who was entitled to fill it. But when he was on the point of receiving the office, another man came before the magistrate and attacked Marinus, saying that as a

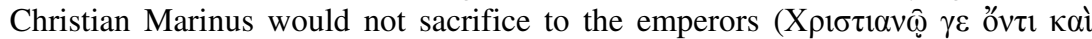

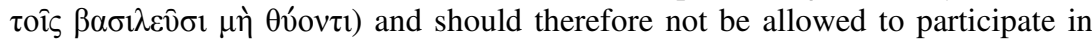
the honours that belonged to the Romans in accordance with their ancient laws, but that the post should fall to himself instead.

It is of some interest that the acta of the trial of Cyprian of Carthage are silent about the imperial cult, except for the archbishop's indirect observation: "We Christians serve this God, we pray to him day and night, for ourselves, for all mankind, for the health of the emperors themselves" (. . . et pro incolumitate ipsorum imperatorum). ${ }^{129}$ These prayers were of course delivered without the customary pagan sacrifices.

By the time of the First Tetrarchy, Christians were serving in the imperial guards, regular army formations and imperial administration. A Christian soldier named Aurelius Mannos is mentioned in a funerary inscription at Eumenia in Phrygia: he was a cavalryman and horse-archer holding the special office a draconarius, "bearer of the dragon standard" in the office of Castrius Constans, who was civil governor of Phrygia and Caria shortly after 293. ${ }^{130}$ Eusebius of Caesarea mentions Adauctus, a senior manager of the imperial estates in the time of Diocletian (magister rei privatae and rationalis summarum), and Dorotheus, a presbyter of the church of Antioch, who was put in charge of the imperial purple dye workshops in Tyre, a politically sensitive position. ${ }^{131}$ It is therefore not surprising to find significant numbers of Christian soldiers mentioned in the martyr acta. One of the matryrologies dating from before the Great Persecution, that of Marcellus (ca. 298?), which exists in two recensions, $\mathrm{M}$ and $\mathrm{N}$, mentions a practice that was implicitly connected with the imperial cult, that of celebrating the birthdays of the reigning emperors. The situation is set forth in quasi-documentary style in recension $\mathrm{M}$, which places the event in Tingis in Mauretania: ${ }^{132}$

In the city of Tingis when Fortunatus was governor, the birthday celebration of the emperor arrived. Thereupon, while everyone was dining at the banquet tables, a certain Marcellus, one of the centurions, refused these profane festivities (profana ... convivia). After throwing down his military belt (cingulum militare) in front of the legionary stands (signa legionis), which were then present, he said: "I am a soldier of Jesus Christ the eternal king" (Iesu Christo regi aeterno militio).

The birthday of the emperors (natalis imperatoris) was presumably celebrated with ad hoc sacrifices. It is not entirely clear from the narrative that Marcellus was compelled to perform the traditional salutes or eat any sacrificial meats. Marcellus is said to have repeated his statement in front of the prefect Anastasius, and

129 Acta Proconsularia S. Cypriani 1, ed. MusuriLlo 1972, 168-169.

130 ILS 8881.

131 Eus. h. e. 8.11 and 7.32 .

132 M. Marcell., recensio M 1, ed. MusURILLO 1972, 250-251. 
to have been tried in front of a certain Aurelius Agricolianus, a deputy of the Prefect of the Praetorian Guard. The report is clarified by a letter found in some manuscripts of recension $\mathrm{N}$ of the $a c t a$, which places the event not in Mauretania but elsewhere, at Leon in the province of Gallaecia, Spain, in the camp of the Legio VII Gemina: ${ }^{133}$

Manlius Fortunatus: a greeting to his Agricolianus. On the most fortunate and blessed day in the whole world of the double birthday of our lords and of the same Augusti (and) Caesares, while we were solemnly celebrating it, O lord Aurelius Agricolianus, Marcellus a regular centurion of the first cohort (centurio ordinarius), seized by what madness I don't know, voluntarily ungirded his military belt, the sword and staff (balteus et spata et vitis) that he was wearing, thought to throw them down in the central space of the camp (principia) of our lords. I thought it necessary to refer this act to your jurisdiction and to have the man himself sent to you.

The charges centred on Marcellus' throwing down the symbols of his military oath (sacramenta). ${ }^{134}$ There is no hint that he was put on trial for directly failing to honour the guardian spirit or majesty of the emperors. The prefect Anastasius' name, if accurately emended in recension $\mathrm{M}$, is a plausibly Christian one. ${ }^{135}$ It seems quite likely that Christian soldiers would normally have participated in the emperor's birthday celebrations, as long as they did not have to perform sacrifice. It should be borne in mind that many of these 'Christians' would as yet have been only catechumens and not yet 'sealed' by baptism. They would therefore have enjoyed marginally greater freedom to participate in these festivals. It is worth noting that Marcellus' alleged statement in recension $M$ about serving Christ as 'eternal king' (rex aeternus) was contumacious, inasmuch as the Tarquinus Superbus had supposedly been placed under damnatio memoriae at the time of the expulsion of the kings from Rome ca. 510 B.C. ${ }^{136}$ It is difficult to say whether these approximated his original words, or are a consequence of later redaction. Either way, they are inconsistent with the conservation of Roman political and

133 M. Marcell., recensio N 3, ed. MusuriLlo 1972, 256-257 n. 17. The brevity of the text in recension $\mathrm{N}$ is perhaps an argument in favour of its relative antiquity, but its dating formula is corrupt, referring to Diocletian and Maximian who resigned in 305 as Augusti, but naming Constantine and Licinius as their Caesars, who together held this rank only between 306-308. A Christian redactor must have inserted the names of the latter two, displacing the names of the actual Caesars ca. 298, Galerius and Constantius I, under whom Christians were persecuted. But this transposition cannot have been made after about 317, when Constantine and Licinius began to have political differences. Recension $\mathrm{N}$ was therefore composed in the first quarter of the fourth century, sometime between 306 and 317. It therefore reflects an early tradition, but one that has been consciously modified. Cf. MUSURILLO 1972, 257 n. 16.

134 M. Marcell., recensio M, ed. MUSURILlo 1972, 250-255.

135 The manuscripts have Astasius > Anastasius.

136 In general, see CORNELL 1995, 215-230, 236-239. In particular, ALFÖLDI 1963, 79. 
religious traditions that typifies the policies of the fourth-century Christian emperors, when the Latin text would have been edited. ${ }^{137}$

The main sequence of martyrologies that are under consideration here reflects the situation after the Fourth Edict of Diocletian in January/February 304. It was, according to Eusebius, a "general law" requiring "that in the cities all the people should sacrifice as a whole and offer libations to the images." 138 If the martyrologies are an accurate reflection of the Christian experience of religious coercion, temples of the imperial cult were seldom involved in these proceedings. For example, in the historically authentic Passio Iuli veterani, there is a single oblique reference to it. The prosecutor Maximus is reputed to have said: "See how stupid you are as one who more fears a dead man [Christ] than kings (sic) who are living." It would have been obvious in the minds of readers that emperors who had been deified after their deaths did receive worship on the principle of do ut des, and that honours to emperors living and dead were a part of the annual religious calendar. ${ }^{139}$

The martyrdom of Dasius appears to have taken place at Durostorum in Moesia Inferior after Diocletian's Fourth Edict of early $304 .{ }^{140}$ The martyrdom narrative reflects a late tradition and there are serious difficulties with the historical details found in it. The ostensible event was the festival of Saturn on the first of January, which in this instance involved even Christians participating in revels and mummery, and their implication in a sacrifice - supposedly involving selfimmolation. ${ }^{141}$ The crucial passage in the text from the standpoint of imperial cult is the advice given by a certain Bassus, legate of the Legio XI Claudia, in which Dasius was serving: ${ }^{142}$

The legate Bassus said: "Pray to the images of our masters the emperors who have provided us with peace, give us our rations and every day show concern for our advantage.... Dasius, supplicate the sacred images which even the barbarian nations revere."

The imperial cult may have proved a convenient vehicle for carrying out Diocletian's Fourth Edict; its shrines lay in most cities, and a sincere reverence for the Tetrarchs may have been widespread because of their successes in pacifying the frontiers and providing rations to the much enlarged army that now manned the state factories and officia of the provincial governors. These sentiments appear

137 E.g. Cod. Theod. 9.16.2 and 9.16.9. TROMBLEY 1993-94, 1:68-70.

138 Eus. mart. Pal. 3.1.

139 Cf. MACMulLEN 1984. On the imperial cult in the religious calendar, see P.Dura 54, quoted in LEE 2000, 16-19.

140 If the narrative has any historical basis, this would put the Fourth Edict at or near the beginning of January. Dasius is reported to have been executed on 20 November, presumably of the same year.

141 The epigraphic evidence suggests that human sacrifice was normally carried out using an animal as a proxy for the designated human 'victim'. FREND 1952, 79f.

142 M. Das. 7-8, ed. MuSURILLO 1972, 276-277. 
frequently in the titulature of inscriptions erected in the Tetrarchs' honour, as for example recorded in an inscription of A.D. 290 at Augusta Vindolicorum in Raetia Secunda (present-day Augsburg) where Diocletian had strengthened the frontier defences through the construction of fortresses and deployment of troops against the Alamanni, Burgundians and Franks. He is characterized as providentissimus princeps, rector orbis ac dominus, fundator pacis aeternae. ${ }^{143}$ This is an authentic feature of the Dasius narrative, even if the existing redaction has a terminus post quem in the late fourth century. ${ }^{144}$ The customs of 'praying' and 'supplication' at imperial shrines may well have been characteristic aspects of the cult throughout the first century of the Christian empire.

An early Christian response to the imperial cult is found in the Contra Celsum of Origen, which appears to have been written, following a statement of Eusebius of Caesarea, during the imperium of Philip the Arab. ${ }^{145}$ It will be recalled in this connection that Emperor Alexander Severus' mother granted an audience to Origen in Antioch ca. 232, an event that has been seen as a feature of trends in syncretism and a supposed 'drift to monotheism'. Whatever Origen's experience of the imperial household, he has nothing good to say about the imperial cult. On the contrary, he sums up the views found in such diverse predecessors of his as Epictetus, Tertullian, Minucius Felix, and the anonymous authors of the martyrdoms of Polycarp and the Scillitani. ${ }^{146}$ He observes: ${ }^{147}$

The doctrine concerning the institution of emperors and rulers is profound. Many questions are raised on this subject by the existence of those who have ruled savagely and tyrannically, or of those who have drifted from exercising rule into debauchery and wantonness. On this account we here omit any discussion of the problem. However, we certainly do not swear by the fortune (genius [tychē]) of the emperor, in the same way as we do not swear by any other supposed god. For if, as some have said, fortune is only a mode of expression like an opinion or a disagreement, we do not swear by something which does not exist as though it were a god, or as if it were a certain reality and had the power to do something, lest we apply the force of an oath to the wrong things. Or if, as some think who say that people who swear by the genius of the Roman emperor are swearing by his daemon ..., in this case also we ought rather to die than to swear by a wicked and faithless daemon which often commits sin with the man to whom it has been assigned, or sins even more than he does.

143 ILS 618 = CIL III 5810. KELLNER 1971, 156f.

144 The use of a precise Trinitarian formula seems characteristic of the time after the Second Oecumenical Council at Constantinople in 381. M. Das. 8, ed. Musurillo 1972, 277.

145 CHADWICK 1965, xiv-xv.

146 Orig. Cels. 8.65 (CHADWICK 1965, 502 with n. 2). At Epict. diss. 4.1.14, we read: "But who can compel me, if not Caesar the lord of all things? Do you not yourself admit that he is one of your masters? Because, as you say, he is the koinos of all things, ... but know that you are the slave of a great household. Just as the people of Nicopolis were accustomed to shout: 'By the tyche of Caesar, were are free men!'” (SCHENKL 1965, 356 lines 17-23).

147 Orig. Cels. 8.65 (quotations are from the translation by CHADWICK 1965). 
Origen shows a deliberate disingenuousness about the imperial cult on one point, for surely he knew that 'bad' emperors suffered damnatio memoriae at the hands of the Senate, a process that led to the removal of their images from public places and from the temples, the erasure of their names from honorific inscriptions and the annulment of their decrees. He had experienced this any number of times in his early years. When one comes to the time of Constantine, however, one sees the gradual displacement of the idea of the emperor's tyche or genius in favour of the Apollonian divus comes as seen on a gold solidus of 313, culminating eventually in Christ, as embodied in the Chi-Rho borne aloft on the labarum. ${ }^{148}$ Origen puts the solar divinities of the era, including the divine companion of the future emperor Aurelian (270-275), Sol Invictus, into a particular context, that of a created being devoid of divine power: ${ }^{149}$

We praise Helios (the sun) as a noble creation of God, which keeps God's laws and hears the saying, "Praise the Lord, sun and moon", and with all its power praises the Father and Creator of the universe.

Origen is at pains to deny the accusation of Celsus that "all earthly things have been given to [the emperor], and whatever you receive in this life you receive from him". He is happy to accept the Homeric dictum "let there be one ruler, one king", while denying the need for the imperial cult. ${ }^{150}$ Specifically, a refusal to honour the tyche or genius of the emperor does not involve a breach of the pax deorum, but is conducive to a more permanent terrestrial peace. Origen envisions that the Romans might "neglect the customary honours to both gods and men" with impunity. In making this point Origen implicitly predicts the coming of a Christian empire, whose fulfilment is reported in a triumphalist section of Augustine's City of God, as will be seen later: ${ }^{151}$

Let [everyone] deny the Homeric doctrine, while keeping the doctrine of the divine right of the king and observing the command "Honour the emperor." Yet on such a basis as this neither would the emperor be left alone [of divine assistance], nor would he be deserted [by the gods], nor would earthly things be in the power of the most lawless and savage barbarians. For if, as Celsus has it, every one were to do the same as I, obviously the barbarians would also be converted to the word of God and would be most law-abiding and mild. And all other worship would be done away and only that of the Christians would prevail. One day it will be the only one to prevail.

Origen concludes his argument with a plea for a pax Romana buttressed by Christian prayers - but not sacrifices - for the welfare of the emperor, and may perhaps allude to the reigning emperor, Philip the Arab: ${ }^{152}$

148 KRAUTHEIMER 1983, 33.

149 Orig. Cels. 8.66 (CHADWICK 1965, 502-503).

150 Hom. Il. 2.205.

151 Orig. Cels. 8.68 (CHADWICK 1965, 505).

152 Orig. Cels. 8.73 (CHADWICK 1965, 509). 
$[\mathrm{H}]$ ow much more reasonable it is that, while others fight, Christians also should be fighting as priest and worshippers of God, keeping their right hands pure and by their prayers to God striving for those who fight in a righteous cause and for the emperor who reigns righteously ...? Moreover, we who by our prayers destroy all daemons which stir up wars, violate oaths, and disturb the peace, are of more help to the emperors than those who seem to be doing the fighting.

Origen's daemonology is generally consistent with that seen in later writers, both Hellenes and Christians, including Porphyry, Iamblichus and, to take one example, Nilus of Ancyra. ${ }^{153}$ Whatever the cogency of Origen's arguments, the public cult of the emperor suffered little diminution from the reign of Philip the Arab down to that of Theodosius the Great. The continuous series of inscriptions strongly suggests this, even if a somewhat different style of interpreting the imperial cult became a feature of the cult under the Christian empire.

The Christian emperors of the fourth century drew back from the more extreme claims of the Tetrarchy about the divine nature of emperors and their offspring who were presented as "begotten by gods and the begetters of gods" (diis genitis et deorum creatoribus). ${ }^{154}$ A good example of this is the reduced claim made by Constantine the Great in the military prayer to the highest divinity composed ca. $321:^{.155}$

We know you as the only God, we recognize you as emperor, we invoke you as an ally, we have gained victories through you, through you we are superior to our enemies, we declare thanks to you for past benefits and we hope for future favours. We are all your suppliants, imploring you to preserve us for the longest time of life, safe and victorious, our emperor Constantine and his God-beloved sons.

Constantine is also said to have given instructions for his full-length portrait with eyes upraised and hands in the orant position to be erected over the gates of palaces in some cities, and for a similar image of his face to be struck on coins. ${ }^{156}$ Eusebius leaves open the question of whether images of this type were installed in shrines of the imperial cult as well. The representation of the Christian emperor with the upward gaze, as though contemplating the highest divinity, was not an innovation, but goes back to the second quarter of the second century, as seen for example in busts of the emperors Hadrian and Marcus Aurelius veiled and wreathed as a priest, in the British Museum and the National Archaeological Museum in Athens. ${ }^{157}$ The motif is also quite common in non-imperial statuary from the second quarter of the second century onward, in the portraiture of

153 TROMBLEY 1993-94, 2:88.

154 ILS $629=$ CIL III 710 .

155 Eus. v. Const. 4.19, adapted from COLEMAN-NORTON 1966, 88.

156 Eus. v. Const. 4.14-15. For a coin of the mint of Nicomedia with the upward gaze, see BROwN 1971, 27.

157 GRANT 1968, pl. 1. KALTSAS 2002, nos. 718 and 720 (Hadrian), 723 (Antinous), 725 (Marcus Aurelius), 732 (Lucius Verus). 
priests, philosophers and intellectuals, and aristocratic youths and women, as seen in statuary discovered at Athens and other parts of southern Greece including the islands. ${ }^{158}$ The colossal head of Constantine in the Basilica of Rome has this tendency, as do coins of his mother Helena and a bronze head of his son Constantius II. ${ }^{159}$ The orant posture makes its appearance in fourth-century Egyptian funerary art and undated graffiti in the catacombs, but has resonances in Celtic religion as the posture of the druid in the act of prophesying or casting a curse. ${ }^{160}$ Whatever the impact of Constantine's images in this form, imperial portraiture reverted thereafter to the direct, piercing gaze previously seen in the images the emperors of the Tetrarchy and some of their predecessors, as for example in the bronze statue of an unidentified Christian emperor at Barletta. ${ }^{161}$

Responses to the cult of worshipping emperors varied with the outlook of particular Christian writers. ${ }^{162}$ The ecclesiastical historian Philostorgius condemned the Christians in Constantinople who offered prayers and sacrifices of incense as he understood it - to an image of Constantine: ${ }^{163}$

This enemy of God accused the Christians of worshipping the image of Constantine standing on a porphyry column with sacrifices, of honouring it by burning incense and lighting lamps, and of offering prayers to it as though it were a god ( $\dot{\omega} \varsigma \varepsilon \varepsilon \hat{\omega})$, and of making supplications to it to fend off misfortunes.

It is likely that Philostorgius is describing what were fairly conventional rituals practiced at imperial shrines, about which few Christians felt compunctions. It is suggestive of continuity in ritual, which could be safely performed in front of the image of a Christian emperor, but not in response to the demands of the Tetrarchs during the Great Persecution. Jerome of Stridon makes a similar observation in an exegesis of Daniel 3:18 in commenting on the three young men who refused to adore the image of Nebuchadnezzar: ${ }^{164}$

Whether it is a statue, as Symmachus says, or a golden image, as others convey, we wish to say that worshippers of God should not adore it. Therefore, the civil governors and prominent men of the world who adore statues and paintings (imagines) of the emperors understand they are doing something which the three boys did not wish to do and thereby pleased God.

Like Philostorgius, Jerome is referring to Christians who should know better than to worship an image. His language is somewhat ambiguous, however, and may

158 KALTSAS 2002, nos. 689, 694, 695, 700-702, 706, 707, 722, 726 (Polemon the philosopher), 729, 730, 735, 752, 758, 760, 775, 787-792, 794, 796, 797. BROWN 1971, 76-77, 93.

159 VOGT 1965, pl. 31, 61, 70.

160 Tac. ann. 14.29-30. Adomnan v. Columbi 2.11-12, 22 = SHARPE 1991, 162f., 171. BROWN 1971, 48-49, 68 (Vatican Museum), 95 (Egypt, 6th cent.), 97 (Egypt, 6th-7th cent.), 111 (6th cent.).

161 Grant 1968, pls. 28, 29, 43, 47. BROWN 1971, 89, 119. VOGT 1965, pl. 69. ElSNER 1998, 69.

162 The following examples are noted by BEURLIER 1891, 285-286 and notes.

163 Philost. h. e. 2.17, ed. BIDEZ - WINCKELMANN 1972, 28.

164 PL 25:507. 
not mean the performance of sacrifice. It may refer instead to performing the act of proskynesis, which was de rigueur in the presence of the emperor, when the viewer was confronted with the imperial image in shrines of the imperial cult or public places. Jerome clearly has in mind upwardly mobile public officials and wealthy men who were prepared to engage in every imaginable form of obeisance to promote their advancement. ${ }^{165}$ Finally, Ambrose of Milan in his Hexaemeron speaks of the importance of the head to the human personality and observes: ${ }^{166}$

The heads of emperors, although molded merely of bronze, and their faces, which are shaped from bronze or marble, are worshipped by mankind.

He goes on to say that the limbs of the human body gravitate around the head as though it were a god (numen). These denunciations, whether direct or implicit, are far from being an absolute condemnation of imperial cult which must have enjoyed the implicit approval of the Christian emperors. Philostorgius' difficulty with res Constantiana was of course his own partisanship of the anti-Nicene cause.

\section{Conclusions}

It seems doubtful that the imperial cult became a source of religious conflict between ca. A.D. 244 and 392. The institution of emperor worship was sufficiently flexible to be adapted to Christian monotheism, where the emperor could no longer be considered divine vis-à-vis the godhead; the emperor acted as its instrument, and enjoyed the assistance of the divinity as his divine companion. The emperor may himself have been the son of a deified father, but he and any children of his were considered God-protected rather than gods in their own right. The parameters of the Constantinian revolution were well suited to the continued maintenance of the emperors' statues in the shrines of the imperial cult. This was dictated by dynastic politics and religious conservatism even in the minds of Christian emperors. There is no unambiguously official evidence of petitions to deified emperors being validated by the performance of sacrifice. Yet certain preChristian appurtenances of cult, including sacrifice, continued in spite of periodic laws coming from the emperors.

It is important to bear in mind that the repression of traditional Mediterranean religion lapsed from the reign of Julian until the early years of Theodosius the Great, ca. 361-381, and that the imperial cult would have undergone little diminution during such a period. The later years of Theodosius marked a decisive turning point; sacrifices and other appurtenances of ritual were banned in the law of 8 November 392 - although the imperial cult is not specifically mentioned and the Euhemerist critique of pagan theogonies intensified in the hands of 
Augustine and Rufinus of Aquileia. ${ }^{167}$ Most priesthoods were abolished, but the list of titles in the law omits the priests of the imperial cult at the provincial and urban levels, the sacerdotalis provinciae and the flamen respectively. ${ }^{168}$ It appears that the cult was allowed to continue for reasons of political necessity. In the fifth century the cult became increasingly otiose in the Latin West as the military power and political influence of the emperors crumbled. The weakening of imperial power eroded the primary evidentiary base for understanding the imperial cult, that is, statuary and dedications to the emperors in the Latin epigraphy. Yet cultic personnel like the flamines are still reported in the early sixth century, and

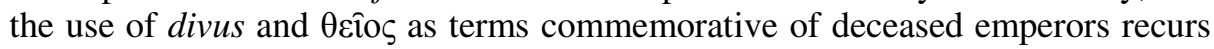
as late as 531-534 in imperial laws referring to Anastasius (Anastasius divae me-

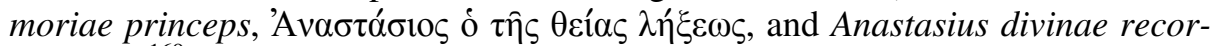
dationis). ${ }^{169}$

These cultic traditions were replaced (rather than displaced) by new ideological constructions, such as those which identified the emperor and his office with Old Testament traditions of sacred kingship. ${ }^{170}$ Practically none of it was promulgated in the epigraphy. It was around this time at the latest, as it appears, that the imperial images of the Principate began to be defaced with crosses. The problem of dating crosses is a particularly important one as regards the continuity of the imperial cult, but it cannot be dealt with here. ${ }^{171}$ One thinks of the cross incised

167 On the religious policies of Theodosius I and the historiographic issues, see ERRINGTON 1997a, and especially ERRINGTON 1997b; LIZZI TESTA 1996.

168 Cod. Theod. 16.10 .14 (7 December 396).

169 Cod. Iust. 4.35.23-24 and 5.27.7, etc. For various fifth-century examples, see BEURLIER 1891, 330-331.

170 Evans 2000, 59-60, 258.

171 I am unaware of any literature on the question of dating crosses, nor are large-scale photographic editions available. For typologies and examples, see SULZBERGER 1926. Trefoil crosses and those with their arms split at the end seem to have come into general use in the Greek epigraphy of Syria I and II and Phoenice Libanensis in the third quarter of the fifth century. A very early example of a trefoil cross is seen at IGLS 1739 (A.D. 412/13), 1726 (?), 1752 (A.D. 453/4), 2068, 2093, 2176, 2204, 2245, 2553, 2632 etc. Crosses with their arms split at the end may begin as early as ca. 460 A.D.; see IGLS 2617, 2618. See also IGLS 258, 270, 271, 276, 298, 309 , $317,589,2622,2630,2648$. This is consistent with the Syriac epigraphy. LITTMANN 1934, nos. 2, 28, 50, 54. Conventional Greek crosses persist after ca. 450. IGLS 1632, and PAES IV D 8, 17, 24, 26, 27, 52. In Asia Minor, crosses with serifs seem to be most common in the thirdfourth century. Trefoil crosses, triangular-tipped crosses, and those with turned-out arms, appear in increasing numbers in the fifth-sixth century. Most of the latter are broadly dated on stylistic grounds and thematic content. One exception is TABBERNEE 1997, no. 84 (A.D. 515). Some Coptic trefoil crosses have third-fourth-century dates assigned to them on the basis of style, e.g. Coptic Museum, Cairo, nos. 4302, 8016, 8556, 8578. In my view, it is doubtful that trefoil crosses developed in Egypt as early as the third century. There is a wide selection of crosses in Syria, many of them datable from inscriptions, that have yet to be studied systematically. PEÑA - CASTEllana - FernandeZ 1983, 237-270. PeÑA - CASTEllanA - FernandeZ 1980, 404 429. There is a weird exception to all this in an inscription from Ma arrat al-Nu'man in Syria II. IGLS 1547. I suspect that the inscription is much later than the late-fourth-century date suggested by its editors. 
on the emperor's gorgoneion on the roundel bust of Marcus Aurelius at Eleusis. The arms of the cross are turned out at their ends, a feature characteristic of the mid-fifth century or later. ${ }^{172}$ The crosses cut on the foreheads of the statues of Augustus and Livia at Ephesus are simple Greek crosses with equidistant arms and can date from any time in the fifth century. The two images also had their noses chiseled off and were broken into small pieces. This most likely took place sometime after the image of Artemis was removed from her temple at the beginning of the fifth century. ${ }^{173}$ The demolition of the temple of the imperial cult excavated at Eretria on Euboia belongs somewhere in this chronological framework, probably at the late fifth century, as indicated above. ${ }^{174}$

The construction of the emperor as the New David and the destruction of previously sacrosanct imperial images symbolically mark the end of the imperial cult qua cultus. ${ }^{175}$

\section{Bibliography}

Alföldi, A., Early Rome and the Latins, Ann Arbor 1963.

Amici, A., Divus Constantinus: le testimonianze epigrafiche, RSA 30 (2000a), 187-216.

Amici, A., Imperatori divi nella decorazione musiva della chiesa di San Giovanni Evangelista, Ravenna studi e ricerche 7.1 (2000b), 13-55.

Barker, E. From Alexander to Constantine: Passages and Documents Illustrating the History of Social and Political Ideas 336 B.C. - A.D. 337, Oxford 1956.

Barnes, T.D., Constantine and Eusebius, Cambridge 1981.

Bayliss, R., Provincial Cilicia and the Archaeology of Temple Conversion (BAR International Series 1281), Oxford 2004.

Beurlier, E., Le culte impérial. Son histoire et son organisation depuis Auguste jusqu'à Justinien, Paris 1891.

Bidez, J. - Winckelmann, F., Philostorgius, Kirchengeschichte, 2d ed., Berlin 1972.

Bonamente, G., Apoteosi e imperatori cristiani, in: Bonamente, G. - Nestori, A. (eds.), I Cristiani e l'Impero nel IV secolo, Macerata 1988, 107-142.

Bowersock, G.W., The Imperial Cult: Perceptions and Persistence, in: Meyer, B.F. - Sanders, E.P. (eds.), Jewish and Christian Self-Definition, vol. 3: Self-Definition in the Graeco-Roman World, London 1982, 171-182.

Brown, P., The World of Late Antiquity AD 150-750, London 1971.

172 Eleusis: personal observation, January 1980. DEUBNER 1937, 75 and pl. 39. TRUMMER 1980, 124-125 and fig. 28. The closest parallels that I have been able to identify belong to the second half of the fifth century. E.g. TABBERNEE 1997, nos. 87 and 88 (both dated by content and style to the fifth-sixth century).

173 SCHERRER 2000, 214-215. Ephesus: personal observation, 22 September 2004. See also TROMBLEY 2008.

174 SCHMID 2001, 140-141.

175 DANIÉLOU 1957. 
Calderone, S., Teologia politica, successione dinastica e consecration in età constantiniana, in: den Boer, W. (ed.), Le culte des souverains dans l'empire romaine (Entretiens sur l'antiquité classique 19), Vandoeuvres - Genève 1973, 215-269.

Cameron, A. - Hall S.G., Eusebius, Life of Constantine, Oxford 1999 [repr. ed. Oxford 2002].

Chadwick, H., Origen, Contra Celsum, 2d ed., Cambridge etc. 1965.

Coleman-Norton, P.R., Roman State and Christian Church, vol. 1, London 1966.

Cornell, T.J., The Beginnings of Rome: Italy and Rome from the Bronze Age to the Punic Wars (c. 1000-264 BC), London 1991.

Creed, J.L., Lactantius, De mortibus persecutorum, Oxford 1984.

Curran, J., Constantine and the Ancient Cults of Rome: The Legal Evidence, $G \& R 43$ (1996), 68-80.

Daniélou, J., David, in: RAC 3 (1957): 594-603.

Deichmann, F.W., Frühchristliche Kirchen in antiken Heiligtümern, JDAI 54 (1939), 105136.

Dessau, H., Über Zeit und Persönlichkeit der Scriptores historiae Augustae, Hermes 24 (1889), 337-392.

Deubner, O., Zu den großen Propyläen von Eleusis, MDAI(A) 62 (1937), 73-81.

Downey, G., The Palace of Diocletian at Antioch, AArch Syr 3 (1953), 106-116.

Drinkwater, J., Maximinus to Diocletian and the 'Crisis', in: Bowman, A.K. - Garnsey, P. - Cameron, A. (eds.), The Cambridge Ancient History, vol. 12: The Crisis of Empire, A.D. 193-337, Cambridge 2005, 28-66.

Elsner, J., Imperial Rome and Christian Triumph, Oxford 1998.

Errington, R.M., Church and State in the First Years of Theodosius I, Chiron 27 (1997a), 21-72.

Errington, R.M., Christian Accounts of the Religious Legislation of Theodosius I, Klio 79 (1997b), 398-443.

Evans, J.A.S., The Age of Justinian: The Circumstances of Imperial Power, London 2000.

Fleischer, R., Der Fries des Hadrianstempels in Ephesos, in: FS F. Eichler, Vienna 1967, 23-71.

Frankfurter, D., Religion in Roman Egypt: Assimilation and Resistance, Princeton 1998.

Frend, W.H.C., The Donatist Church, Oxford 1952.

Gascou, J., Le rescrit d'Hispellum, MEFR 79 (1967), 609-659.

Gizewski, C., Damnatio memoriae, in: Brill's New Pauly: Encyclopaedia of the Ancient World, vol. 4, London 2004, $61 \mathrm{f}$.

Gradel, I., Emperor Worship and Roman Religion, Oxford 2002.

Grant, M., The Climax of Rome, London 1968.

Hanson, R.P.C., The Transformation of Pagan Temples into Churches in the Early Christian Centuries, Journal of Semitic Studies 23 (1978), 257-267.

Hefele, C.J., Histoire des conciles d'après les documents originaux, edited and translated by H. Leclercq, vol. 1.1, Paris 1907, 221-264.

Horstkotte, H., Heidnische Priesterämter und Dekurionat im vierten Jahrhundert n. Chr., in: Eck, W. (ed.), Religion und Gesellschaft in der römischen Kaiserzeit (FS F. Vittinghoff), Köln - Vienna 1989, 165-183.

Jones, A.H.M, The Later Roman Empire 284-602: A Social Economic and Administrative Survey, vol. 1, Oxford 1964. 
Kaltsas, N. (ed.), Sculpture in the National Archaeological Museum, Athens - Los Angeles 2002.

Kellner, H.-J. (ed.), Die Römer in Bayern, Munich 1971.

Körner, C., Philippus Arabs. Ein Soldatenkaiser in der Tradition des antoninisch-severischen Prinzipats (Untersuchungen zur antiken Literatur und Geschichte 61), Berlin 2002.

Kornemann, E., Zur Geschichte der antiken Herrscherkulte, Klio 1 (1902), 51-146.

Krautheimer, R., Three Christian Capitals: Topography and Politics. Rome, Constantinople, Milan, Berkeley - Los Angeles - London 1983.

Lampe, G.W.H., A Patristic Greek Lexicon, Oxford 1961.

Lee, A.D., Pagans and Christians in Late Antiquity, London 2000.

Lepelley, C., Les cités de l'Afrique romaine au Bas-Empire I. La permanence d'une civilisation municipale, Paris 1979.

Littmann, E. (ed.), Syria: Publications of the Princeton University Archaeological Expeditions to Syria in 1904-1905 and 1909, division 4: Semitic Inscriptions, sec. B: Syriac Inscriptions, Leiden 1934.

Lizzi Testa, R., La politica religiosa di Teodosio. Miti storiografici e realità storica, $R A L$ 7th ser. 9a (1996), 323-361.

Macaronas, C.J., The Arch of Galerius at Thessaloniki (Institute for Balkan Studies 113), Thessaloniki 1970.

MacMullen, R., Christianizing the Roman Empire, New Haven 1984.

MacMullen, R., Christianity and Paganism in the Fourth to Eighth Centuries, New Haven 1997.

Millar, F., The Imperial Cult and the Persecutions, in: den Boer, W. (ed.), Le culte des souverains dans l'empire romaine (Entretiens sur l'Antiquité classique 19), Vandoeuvres - Geneva 1973, 145-175.

Mommsen, T., Observationes Epigraphicae, vol. 19: Album Ordinis Thamugadensis = CIL Suppl. 3, Berlin - Rome 1877.

Musurillo, H., Acts of the Christian Martyrs, Oxford 1972.

Nauck, A., Porphyrii Opuscula Tria, Leipzig 1860.

Parker, H.M.D., A History of the Roman World AD 138 to 337, London 1958.

Peña, I. - Castellana, P. - Fernandez, R., Les reclus syriens, Milan 1980.

Peña, I. - Castellana, P. - Fernandez, R., Les cénobites syriens, Milan 1983.

Price, S.R.F., Between Man and God: Sacrifice in the Roman Imperial Cult, JRS 70 (1980), 28-43.

Price, S.R.F., Rituals and Power: The Roman Imperial Cult in Asia Minor, Cambridge 1984.

Rodgers, B.S., Divine Insinuation in the Panegyrici Latini, Historia 35 (1986), 69-104.

Rolfe, J.C., Ammianus Marcellinus, Res gestae, 3 vols. (Loeb Classical Library 300, 315, 331), Cambridge - London 1935-1940.

Schenkl, H., Epicteti Dissertationes ab Arriani Digestae, Stuttgart 1965.

Scherrer, P. (ed.), Ephesus: The New Guide, Istanbul - Selçuk 2000.

Schmid, S.G., Worshipping the Emperor(s): A New Temple of the Imperial Cult at Eretria and the Ancient Destruction of Statues, Journal of Roman Archaeology 14 (2001), 113-142. 
Shahîd, I., Rome and the Arabs: A Prolegomenon to the Study of Byzantium and the Arabs, Washington 1984.

Sharpe, R., Adomnan of Iona, Life of St. Columba, London 1991.

Spieser, J.-M., La christianisation des sanctuaries païen en Grèce, in: Jantzen, U. (ed.), Neue Forschungen in griechischen Heiligtümern, Tübingen 1976, 309-320.

Sulzberger, M., Le symbole de la croix et les monogrammes de Jésus chez les premiers chrétiens, Byzantion 2 (1926), 337-448.

Tabbernee, W., Montanist Inscriptions and Testimonia: Epigraphic Sources Illustrating the History of Montanism, Macon 1997.

Trombley, F.R., Hellenic Religion and Christianziation ca. 370-529 A.D., 2 vols. (Religions in the Graeco-Roman World 115), Leiden etc. 1993-1994.

Trombley, F. R., The Destruction of Pagan Statuary and Christianization (fourth-sixth c[enturies] C.E.), in: Eliav, Y.Z. - Friedland, E.A. - Herbert, S. (eds.), The Sculptural Environment of the Roman Near East: Reflections on Culture, Ideology and Power (Interdisciplinary Studies in Ancient Culture and Religion 9), Louvain 2008, 139160.

Trummer, R., Die Denkmäler des Kaiserkults in der römischen Provinz Achaia (Dissertationen der Universität Graz 52), Graz 1980.

Vogt, J., The Decline of Rome: The Metamorphosis of Ancient Civilization, trans. J. Sondheimer, London 1965.

Wachsmuth, C., Inschrift aus Alexandria, RhM 28 (1873), 581-585. 


\title{
Einziehung und Nutzung von Tempelgut durch Staat und Stadt in der Spätantike
}

\author{
GIORGIO BONAMENTE
}

In der Antike war die Besitzergreifung der spolia die natürliche Folge eines Sieges. Dieser Logik entsprach auch der Übergang von der traditionellen zur christlichen Religion, was durch die Eigentumsübertragung von Tempelgütern und dem größten Teil der Tempel selbst deutlich wird. ${ }^{1}$ In der Tricennalienrede, im Jahre 336 zum 30. Regierungsjubiläum des Kaisers verfasst, vertrat Eusebios eine Ansicht, deren Wurzeln eher in der römischen Tradition als in der christlichen Lehre zu suchen sind, nämlich dass der Kaiser, nachdem er die alten Götter besiegt hatte, in seiner Funktion als $\mu \varepsilon \gamma \alpha \dot{\lambda} \lambda$ ov $\beta \alpha \sigma \imath \lambda \varepsilon ́ \omega \varsigma$ eigentums unter die Soldaten des siegreichen Gottes vornehmen durfte. ${ }^{2}$ In einer ideologisch bedeutenden Rede, in der die felicitas eines Kaisers gefeiert wurde, der für sich den mächtigeren Gott gewählt hatte, zeichnete Eusebios erstmals ein zusammenfassendes, aber genaues Bild von dem Schicksal, das Konstantin den heidnischen Tempeln bereitet hatte: einige von ihnen wurden geschlossen, vor allem aber erfolgte die Beschlagnahmung wertvoller Gegenstände und Materialien sowie der Statuen aller bestehenden Tempel, ${ }^{3}$ zum Beweis seiner Ablehnung traditioneller Kulte und seiner Überzeugung, dass diese im Reich keine Rolle mehr spielen sollten. ${ }^{4}$

Wenige Jahre später legte die Vita Constantini, auch wenn sie die Loslösung des Kaisers von den heidnischen Kulten nachdrücklich betonte, eine Reihe von Zeugnissen im Zusammenhang mit der konsequenten Religionspolitik Konstan-

1 Von LasaulX 1856; Deichmann 1939, 105-136; Deichmann 1954, 1228-1241; NOETHLICHS 1971; HANSON 1978, 257-267; METZLER 1981, 27-40; BONAMENTE 1992, 171 201; KleIN 1995, 129-151; Testa 1991, 311-326; MeIER 1996, 363-376; TSAFRIR 1998, 205 ff.; FOSCHIA 2000, 413-434; DE GIOVANNI 2003, 193 ff..

2 Eus. l.C. 7,13. Die Rede wird auf den 25. Juli 336 datiert; vgl. DrAKE 1975, 345-356; DrAKE 1976, 30 ff.; AMERISE 2005, 18.

3 Das achte Kapitel der zu den tricennalia gehaltenen Rede umreißt die , antiheidnische' Politik des Kaisers (Entscheidung, den negativen Einfluss der traditionellen Kulte zu unterbinden, systematische Registrierung der Tempelgüter, Entfernung kostbarer Materialien, insbesondere der Statuen, Zerstörung des Aphrodite-Tempels in Aphaka). Dieser Abschnitt ist zusammen mit einem Operationsplan in den Kapiteln 3,54-55 der Vita Constantini enthalten. Vgl. BONAMENTE 1992, 179 ff.; BARNES 1989a, 94 ff.; CAMERON - HALl 1999, 15-16; 302 ff.; MARAVAL 2001, 130.

4 Siehe auch GIRARDET 1998, 45 ff. 
tins vor. Bei der geschichtlichen Rekonstruktion muss die literarische Gattung des Werks berücksichtigt werden und es muss zwischen den Aussagen allgemeiner Art und der genauen Beschreibung einer begrenzten Anzahl von Fällen, in denen der Kaiser eingegriffen hatte, unterschieden werden. Zwei parallele Textebenen in Eusebios' Ausführungen erweisen sich nämlich sowohl in Bezug auf das Opferverbot (im Prinzip galt es für alle, ${ }^{5}$ effektiv verbindlich war es jedoch nur für die in den Verfügungen ausdrücklich genannten Empfänger) ${ }^{6}$, als auch in Bezug auf das Verbot der Magie und der verschiedenen Formen der Wahrsagerei (wie die Befragung der Orakel und der Haruspices) $)^{7}$, und im Zusammenhang mit Plünderung, Schließung und Zerstörung der heidnischen Tempel als nützlich: Für letztere sind die Unterschiede zwischen Grundsatzerklärungen, aus denen man eine systematische Zerstörung ableiten könnte, ${ }^{8}$ und der genauen Erzählung der weni-

5 Eus. v.C. 2,45,1 (,,.. von denen das eine [Gesetz] den abscheulichen Götzendienst verbot... keiner sollte es mehr wagen... noch zu opfern“); 4,25,1 (es wird darüber berichtet, ,daß er in rasch aufeinander folgenden Gesetzen und Erlassen allen verbot, den Götzen zu opfern, Wahrsagerei zu treiben, Götzenbilder aufzustellen..."). Auch im Kapitel 4,39 wird zwischen dem konkreten Fall der Stadt Constantina in Phönikien, ,,deren Einwohner unzählige Götzenbilder dem Feuer übergeben und das Gesetz des Heiles dafür eingetauscht hatten“, und der allgemeinen Nachricht, laut der ,,auch in anderen Provinzen ... die Einwohner scharenweise von selber zur Erkenntnis des Heiles [kamen] und sie in Stadt und Land als leeres Nichts [vernichteten], was ihnen früher für heilig gegolten hatte, ... ; ihre Tempel und Heiligtümer, die sich stolz erhoben, zerstörten sie, ohne daß es ihnen jemand befohlen hätte, erbauten dafür von Grund auf Kirchen“, unterschieden. Zur Frage, ob Konstantin tatsächlich ein Opferverbot erlassen hatte, vgl. BARNES 1981, 210 ff.; BARNES 1984, 71 f.; BRADBURY 1994, 123 ff.; ERRINGTON 1988, 309 ff.; BRINGMANN 1995, 42; De Giovanni 2003, 161 ff.; OnidA 2003, $121 \mathrm{ff}$.

6 Eus. v.C. 2,44 (Opferverbot für die Statthalter der Provinzen und praefecti praetorio); 45,1 (allgemeine Anordnung gegen die Kulte und insbesondere gegen die Opfer); 3,48,2 (Opferverbot für Konstantinopel); 4,23 (bezugnehmend auf das allgemeine Opferverbot werden die Statthalter der Provinzen dazu verpflichtet, den Sonntag zu ehren und die christlichen Feste zu feiern); 4,25,1 (generelles Opferverbot). Vgl. BRingmanN 1995, 42; CAMERON - HALl 1999, 20.

7 Vgl. Cod. Theod. 9,16,1-3; 16,10,1. Hierbei müssen sowohl Bedürfnisse der öffentlichen Ordnung als auch die feindliche Einstellung der Christen gegenüber der Wahrsagerei berücksichtigt werden. Vgl. hierzu BARB 1963, 113 ff.; DE GIOVANNI 2003, 31-36.

8 Eus. v.C. 3,54,2 (,So wurden in den einzelnen Städten die Vorhöfe ihrer Tempel ganz mit Recht bloßgelegt, da sie auf Befehl des Kaisers ihrer Tore beraubt wurden. Bei andern wurden die Dachziegel weggenommen ...; wieder bei andern wurden die verehrten Erzbilder ... auf allen Plätzen der Kaiserstadt offen ausgestellt“); 3,57,1 (,Alle, die früher den Götzen gedient hatten, sahen so mit eigenen Augen, wie ihr Wahn widerlegt wurde, und sie schauten überall die tatsächliche Vereinsamung ihrer Tempel und Götzenbilder."); 4,39,2 (,auch in anderen Provinzen ... vernichteten [sie] in Stadt und Land ... ihre Tempel und Heiligtümer ... ohne daß es ihnen jemand befohlen hätte“). Von einer allgemeinen Schließung der Tempel sprechen auch Hieronymus und Orosius (vgl. Anm. 45). Der wichtigste Text ist jedoch Eus. v.C. 4,25,1 (es wird darüber berichtet, ,daß er in rasch aufeinander folgenden Gesetzen und Erlassen allen verbot, den Götzen zu opfern, Wahrsagerei zu treiben, Götzenbilder aufzustellen ... oder mit blutigen Gladiatorenkämpfen die Städte zu beflecken“), der für die Forschung einen allgemeinen Bezugspunkt für die erhaltenen constitutiones und insbesondere für das grundlegende Gesetz von Constantius II. (Cod. Theod. 16,10,2) darstellt (...legem divi principis parentis nostri); vgl. WARMington 1993, 201 ff.; CUNeO 1997, 88-89; De Giovanni 2003, 93 ff.; 114 ff.; 94 ff.; 
gen tatsächlich erfolgten Zerstörungen unter Konstantins Herrschaft noch deutlicher. $^{9}$

Was die Tempelgebäude und das dazugehörige Inventar betrifft, liefern zwei Passagen bei Eusebios konkrete Hinweise, dass eine geringe Anzahl von Tempeln auf Initiative des Kaisers zerstört und Statuen der heidnischen Kulte zur Stadtverschönerung verwendet wurden, was deren offensichtliche Profanierung in Rom und in anderen Städten des Reichs, vor allem in Konstantinopel, zur Folge hatte, ${ }^{10}$ aber vor allem darüber, dass die Tempelinventare im ganzen Reich systematisch beschlagnahmt wurden, worüber uns das umfassendste und genaueste Zeugnis durch Eusebios überliefert ist. ${ }^{11}$ Aufgrund des beachtlichen Umfangs und der weitreichenden Bedeutung dieses komplexen Vorgangs erscheint es angebracht, zuerst die Politik der christlichen Kaiser über die Tempel und das dazugehörige Inventar zu untersuchen.

Es scheint, dass im Jahre 336, als Eusebios seine Festrede vortrug, ein Programm zur Konfiskation der Tempelinventare bereits abgeschlossen oder zumindest weit fortgeschritten war. Eusebios umreißt dabei auch die behördlichen $\mathrm{Zu}$ ständigkeiten und Vorgehensweisen und beschreibt folgende Phasen: ${ }^{12}$

CAMERON - HALl 1999, 44-45 (über das Nebeneinanderbestehen einer effektiven Toleranz und einer, verbalen' Feindseligkeit gegenüber dem Heidentum bei Konstantin).

9 In der Laus Constantini wird von der Zerstörung des Aphrodite-Tempels in Aphaka unter Mitwirkung des Heers berichtet (Eus. l.C. 8,7 = Eus. v.C. 3,55,5), während im entsprechenden Kontext der Vita Constantini die Zerstörung eines zweiten Aphrodite-Tempels in Baalbek (Heliopolis) und des Asklepios-Tempels in Aigai in Kilikien erwähnt wird: Eus. v.C. 3,56-58 (vgl. Soz. h.e. 5,10; Zon. 13,12,30-34; LEPPIN 1999, 479). Hinzu kommt der Abriss des AphroditeTempels auf dem Areal der Grabeskirche in Jerusalem: Eus. v.C. 3,26,7. Das erste Kapitel des dritten Buchs verdient Aufmerksamkeit, in dem ein allgemeiner Eindruck von der christenfreundlichen Religionspolitik Konstantins vermittelt wird; was jedoch das Schicksal der Tempel betrifft, wird ausdrücklich erwähnt, er ,ließ das Material, das an den Geschenken bisher zu nichts gedient hatte, für immer denen geben, die es verwerten konnten ... er zerstörte von Grund auf gerade diejenigen (scil. Göttertempel), die bei den Götzendienern in höchster Ehre standen“ (3,1,5). Soz. h.e. 2,5,2-3 greift die Nachrichten des Eusebios wieder auf und spricht von einem Plan des Kaisers, der darauf ausgerichtet war, die Tempel und Kultstatuen zu verachten, kostbare Materialien und Statuen aus Edelmetall zu beschlagnahmen und die Bronzestatuen zur Verschönerung Konstantinopels zu verwenden. Hinsichtlich der Zerstörung der Tempel nennt jedoch auch er nur den Asklepios-Tempel in Aigai und den Aphrodite-Tempel in Aphaka (Soz. h.e. 2,5,5). Sporadische weitere Fälle sind für Rom, Konstantinopel, Alexandria und Antiochia bezeugt; vgl. DEICHMANN 1939, nn. 1; 4; 21; 25; 27; METZLER 1981, 30-31; STEMBERGER 1987, 49 ff.; HAHN 2004, 22 ff. (dabei muss berücksichtigt werden, dass Eusebios den Fällen in Palästina besondere Aufmerksamkeit schenkt). Vgl. DE GIOVANNI 2003, 118-119.

10 Soz. h.e. 2, 5, 3-4; vgl. MANGO 1963, 55 ff.; DEICHMANN 1975; BARSANTI 1992, 115-150; STEWART 1999, 159 ff.

11 In den Kapiteln 54 und 55 des dritten Buches der Vita Constantini wird der entsprechende Abschnitt der Laus Constantini wiedergegeben; dies ermöglicht, einen genauen terminus ante quem festzulegen und vor allem über eine beinahe zeitgenössische Überlieferung zu verfügen; vgl. BONAMENTE 1992, $180 \mathrm{ff}$.

12 Vgl. auch Soz. h.e. 2,5,2-3; METZLER 1981, 28-29; SABBAH 1983, 72; BonAMENTE 1992, 198-199. 
- Der Kaiser entsandte in jede Provinz eine aus zwei Personen seines Vertrauens bestehende Delegation (Eus. l.C. 8,2-3 = v.C. 3,54,5-6).

- Im ganzen Reich wurden alle Statuen der heidnischen Kulte gezählt: „Sie befahlen den Priestern, ..., selber ihre Götter aus den dunklen Winkeln ans Licht hervorzubringen“" (Eus. l.C. $8,3=$ v.C. 3,54,6) $)^{13}$.

- Die Statuen wurden ihrer Wertgegenstände beraubt (Eus. l.C. 8,3 = v.C. 3,54,6).

- Alle wiederverwendbaren Materialien wurden konfisziert und eingeschmolzen (Eus. l.C. $8,3=$ v.C. 3,54,6).

- Überflüssiges und unbrauchbares Material überließen sie ,zum Andenken an ihre Schmach den Götzendienern." (Eus. l.C. 8,3 = v.C. 3,54,6). ${ }^{14}$

- Es wurden alle Bronzestatuen beschlagnahmt (Eus. l.C. 8,4 = v.C. 3,54,7).

Eusebios' Zeugnis wird durch spätere Dokumente im Laufe des 4. Jahrhunderts bestätigt und bleibt der Bezugspunkt für das Schicksal der Tempelgüter, oder wie Libanios es formelhaft und wirkungsvoll ausdrückt, um Konstantin den Großen zu beschreiben: ,der Kaiser, der die Tempel ihrer Inventare beraubt hat“ ${ }^{\text {" }}{ }^{15}$ Es ist nicht bekannt, wie hoch der Anteil der Tempel ist, die bereits der Inventarisierung unterzogen worden waren, oder welche räumliche Verbreitung das Phänomen erreicht hatte, als Eusebios in der Tricennalienrede darüber berichtete; von vielen heidnischen Tempelschätzen sind Zeugnisse aus Zeiten lange nach der konstantinischen Herrschaft erhalten, ${ }^{16}$ und in jedem Fall müssen die unterschiedlichen Situationen in Städten wie Rom (wo der Wunsch nach dem Weiterbestand der religiösen Tradition ausgeprägter war), ${ }^{17}$ Konstantinopel und im allgemeinen in den großen Städten, in denen die hellenistische Kultur stärker verwurzelt war, wie Antiochia, caput Syriae et Orientis, ${ }^{18}$ und Alexandria ad Aegyptum - letztere mit

13 Vgl. Soz. h.e. 2,5,2-3.

14 Die Tatsache, dass in beiden Texten die Gläubigen, die die Götzenbilder als Kultgegenstände betrachteten, als ,,abergläubig“ bezeichnet werden, deutet darauf hin, dass versucht wurde, sie mit dieser Propaganda auszulöschen.

15 Lib. or. 30 (pro templis),37; vgl. Anm. 24.

16 Man bedenke die Gottlosigkeit Serenas, die die Halskette der Kultstatue der Magna Mater in Rom für sich beanspruchte (Zos. 5, 38, 3, siehe dazu Anm. 110) und die wiederholten Plünderungen des Sarapeions in Alexandria. Zur Frage, ob es sich eventuell um Objekte handelt, die nach Konstantin, vielleicht sogar unter Julian, neu gestiftet worden waren, siehe Anm. 80-82.

17 Im Jahre 340 ließ der praefectus urbi Fabius Titianus den Romulus-Tempel restaurieren und das Forum mit einer Reihe von Statuen schmücken, die die gleiche Inschrift tragen: Fabius Titianus, v.c., consul, praef. urbi, curavit: CIL V, 1653; 31879; 31880; 37107; 37108. Vgl. CHASTAGNOL 1962, 108 ff.; LIZZI TESTA 2001, 671 ff. Zu den späteren Aktivitäten der heidnischen Senatoren in Rom vgl. Anm. 91 und 107.

18 Lib. or. 11,125; 15,53; Petit 1955, 191 ff.; FeStugiere 1959; LiEBESCHuetz 1972; CRACCO RUGGINI 1980, 15-101; HAHN 2004, 130 ff. 
einer außergewöhnlich hohen Anzahl von Kultstätten, unter denen das Sarapeion besonders herausragte - berücksichtigt werden. ${ }^{19}$

Für die Operationen finanzieller Art in Verbindung mit diesem politischen Programm wurde die res privata als Fonds genutzt, ${ }^{20}$ sowohl wegen der direkteren Abhängigkeit vom Kaiser als auch wegen des komplizierten juristischen und administrativen Rahmens: Die Tempel waren zwar ein öffentliches Gut, ${ }^{21}$ nicht jedoch die Kirchen, so dass die Verfahren, die notwendig waren, die Tempelgüter einerseits der Öffentlichkeit zur Verfügung zu stellen, und andererseits den Kirchen zu übertragen und ihnen damit ihre Bestimmung als öffentliches Gut zu entziehen, völlig unterschiedlicher Art waren. In diesem Zusammenhang muss betont werden, dass die Politik Konstantins darauf ausgerichtet war, die Tempelgüter generell und in systematischer Form zu profanieren, während die direkte Eigentumsübertragung von Tempeln und ihrer Inventare auf die Kirchen auf wenige Ausnahmen beschränkt blieb. ${ }^{22}$

Obwohl Konstantins Gesetze bezüglich der Tempelinventare eine langsame und unvollkommene Umsetzung erfuhren, charakterisieren sie seine Religionspolitik und im speziellen Fall, in welcher Form er das Heidentum gesetzlich einschränken wollte: Im Mittelpunkt stand dabei nicht die Schließung der Tempel, sondern ein drastischer Eingriff in ihre finanziellen Ressourcen ${ }^{23}$ und die Schädigung ihres Ansehens - Aspekte, die in den antiken Quellen hervorgehoben werden und in der neueren Forschung Bestätigung finden. ${ }^{24}$

19 Im Jahre 350 sah der Verfasser des Textes Expositio totius mundi et gentium (36) noch in der ganzen Stadt die heidnischen Kulte in voller Blüte; Libanios bezeichnete Alexandria als „Stadt des Sarapis“" (Lib. or. 30, 35); zum Zeitpunkt der Zerstörung im Jahre 392 besaß das Sarapeion noch zur Verzierung der Statuen Edelmetalle, die Kaiser Theodosius einschmelzen ließ, um sie der Kirche von Alexandria zur Versorgung der Armen zur Verfügung zu stellen. Vgl. Soz. h.e. 5,16; SCHWEITZER 1991, 164 ff.; HAHN 2004, 22 ff.; 95 ff.; HAHN 2008; vgl. Anm. 105.

20 Als Konstantin bereits im Jahre 313 dem Bischof von Karthago 3000 folles über den rationalis Africae Ursus zukommen ließ, stellte er dem Bischof auch den procurator rei privatae Heraclides zur Verfügung: unten Anm. 53.

21 Zur Kontinuität dieses Prinzips vgl. Sex. Pomp. Fest. De verborum signif. 284L: publica sacra:

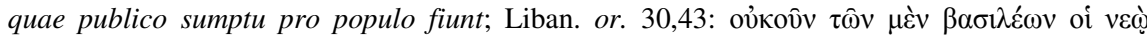

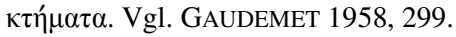

22 Deichmann 1954, 1229, mit Bezug auf Cod. Theod. 10,3,4-5; Cod. Iust. 11,70,4.

23 Einige Tempel Konstantinopels (der Gottheiten Helios, Artemis, Selene und Aphrodite) wurden ihres Inventars beraubt; vgl. Jo. Mal. chron. 317; Thphn. chron. a. m. 5810 (er vermerkt, dass ein Teil der Spenden an das Sarapeion der Kirche zugeleitet wurden); DAGRON 1974, $347 \mathrm{ff}$.

24 Besonders prägnant ist ein Zitat des Libanios (Lib. or. 62,8 = 4,350,6-11 Förster), der die Poli-

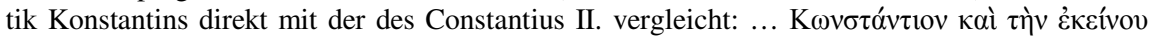

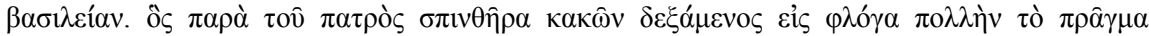

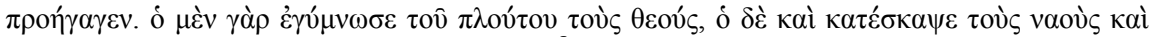

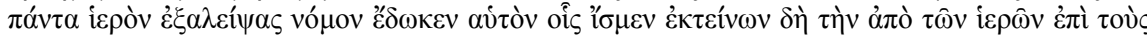

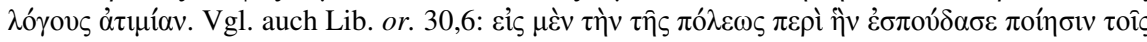

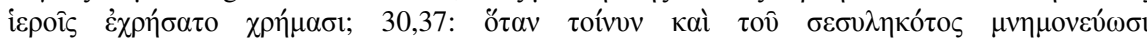

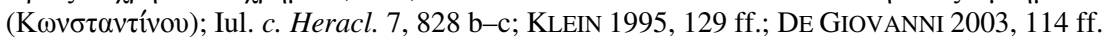


Die konkreten Auskünfte, die uns Eusebios mit seiner Rede liefert, von denen anzunehmen ist, dass sie einen wahrheitsgetreuen Spiegel der Politik Konstantins darstellen, entkräften jeden antiken und neuzeitlichen Verdacht über die Unsicherheit oder sogar Doppeldeutigkeit seiner Religionspolitik, der sich auf die Überzeugung gründet, dass das Fehlen einer Anordnung der generellen SchlieBung der heidnischen Tempel als ein Zeichen von Eklektizismus und Kompromissbereitschaft zu deuten sei. Konstantin ließ in der Tat wenige Tempel zerstören oder schließen und wenige christliche Kirchen an Stelle heidnischer Tempel errichten (vor allem in Palästina) ${ }^{25}$, und ordnete keineswegs die allgemeine Schließung der Tempel an; sein ausdrücklicher Wunsch war es, dass sie auch in Zukunft Besuchern zur Verfügung stehen sollten. Diese Linie wurde durch eine Reihe von Bestimmungen nach dem Sieg über Licinius bestätigt, die Grundlage seiner Religionspolitik nach dem Konzil von Nizäa blieben. ${ }^{26}$

Folgerichtig erscheint in diesem Zusammenhang der Fortbestand der Zeremonien und Strukturen in Verbindung mit dem Herrscherkult. Diese wichtige Institution hatte eine primäre Funktion als Gelegenheit des offiziellen Ausdrucks der Loyalität gegenüber dem Kaiserhaus, und Konstantin war darauf bedacht, das gefestigte Gleichgewicht nicht aus dem Lot zu bringen. In diesem Bereich muss die Politik der ,christlichen ' Kaiser langfristig betrachtet werden und es muss berücksichtigt werden, dass die Zeremonien und die Organisation des Kaiserkults erst im darauffolgenden Jahrhundert aufgehoben wurden, wie aus dem Edikt Cod. Theod. 16,10,20 vom 30. August 415 hervorgeht, in dem die Abschaffung der Ämter der sacerdotales angeordnet wird. ${ }^{27}$

Ein wichtiges Dokument für Konstantin den Großen ist der kaiserliche Erlass von Hispellum, eine hinsichtlich Chronologie und Inhalt zwar sehr umstrittene Quelle, die jedoch beispielhaft die Ausgewogenheit seiner Religionspolitik verdeutlicht: Dem concilium der Umbrer wurde gestattet, ein eigenes Zentrum für den Kult der domus Augusta und damit ein neues Heiligtum, das für den Herrscherkult bestimmt war, zu errichten. Konstantin legitimierte zwar die Versammlungen des concilium und die Tätigkeiten des coronatus, verbot allerdings, Opfer darzubringen. ${ }^{28}$ In diesem Zusammenhang muss die Formulierung in der Vita

$25 \mathrm{Zu}$ den Tempeln von Aphaka, Aigai und Heliopolis, von Mambre und des Areals der Grabeskirche von Jerusalem, vgl. Anm. 9.

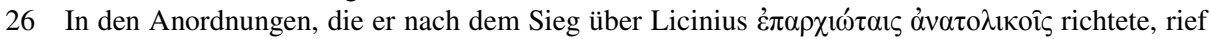
er zum Prinzip der Toleranz gegenüber den Personen auf, die „sich auf dem Irrweg befinden“ (Eus. v.C. 2,26,1-2; 2,59-60,2). Hierin stimmen die antiken Quellen, ob christlichen oder heid-

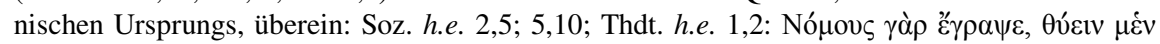

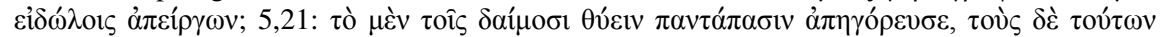

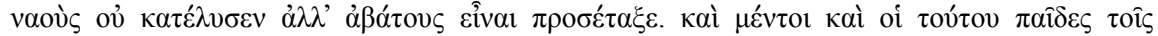

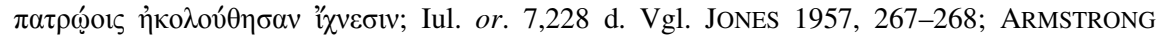
1984, 1-17; GAUDEMET 1990, 451-452.

27 Vgl. Anm. 126.

28 Das im Erlass von Hispellum enthaltene Opferverbot entspricht dem, das den Statthaltern der Provinzen und den praefecti praetorio auferlegt wurde und auf das die Vita Constantini Bezug 
Constantini, die einen Eindruck der vollkommenen Distanzierung vom Herr-

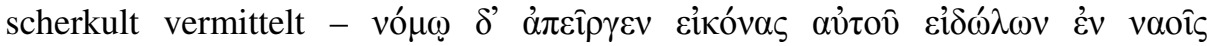

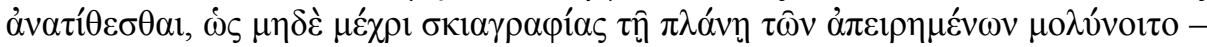
vor dem Hintergrund einer Notiz des Kirchenhistorikers Sokrates betrachtet werden, nach der Konstantin Bildnisse von sich selbst in den Tempeln anbringen ließ. ${ }^{29}$

Dieselbe Vita Constantini verdeutlicht nämlich auch, dass die Politik des Kaisers fallorientierter und realitätsbezogener war als die zitierte und andere Grundsatzerklärungen glauben lassen mögen: In der Quelle wird ein Edikt aus dem

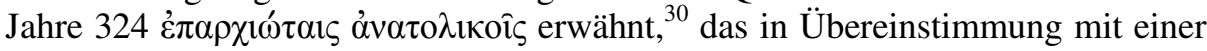
der oben erwähnten Anordnungen (die Rückgabe der Kultstatuen an die Götzendiener) den freien Zugang zu den Tempeln vorsah ${ }^{31}$ und daher als ein Zeugnis einer toleranten Religionspolitik (oder besser: in der Ausübung seiner Rolle als pontifex maximus) hervorgehoben werden muss. Auch wenn Konstantin erklärte, dass er die traditionellen Zeremonien für superstitio hielt und er die Heiden öffentlich ihrer Irrtümer beschuldigte (oi $\pi \lambda \alpha v \omega ́ \mu \varepsilon v o l$; oi $\delta$ ' $\dot{\varepsilon} \alpha v \tau$ ov̀ $\alpha^{\prime} \varphi \dot{\varepsilon} \lambda \kappa o v \tau \varepsilon \varsigma$ ), erließ er zwei wichtige Bestimmungen zur Nutzung der Tempel und ihrer Inventare:

- die Tempel standen weiterhin den Gläubigen zur Verfügung (Eus. v.C. $2,56,2)$

- die „traditionellen Tempelzeremonien“ waren nicht allgemein verboten und der Kaiser dementierte Behauptungen dieser Art (Eus. v.C. 2,60,2).

Die Anpassung der Entscheidung an die jeweilige konkrete Situation und die Vorsicht, die Konstantin bei seiner Politik walten ließ, unterstreichen die historische Bedeutung der allgemeinen Einziehung der Tempelinventare, von der uns Eusebios das genaueste, aber nicht das einzige Zeugnis liefert, und die nicht durch Doppeldeutigkeit gekennzeichnet ist. Konstantin verfolgte seine religionspolitischen Ziele auf unverkennbare Art - entschlossen und stufenweise. Aus dieser Perspektive war die Beschlagnahme der Tempelgüter eine Tat von epochaler Bedeutung.

Firmicus Maternus stellte die Bedeutung der Konfrontation mit dem Heidentum von seinem Standpunkt aus dar und sah in der Beschlagnahmung der Tem-

nimmt (siehe oben). Zum Erlass vgl. ANDREOTTI 1964, 249 ff.; GASCOU 1967, 609 ff.; SALZMAN 1987, 172 ff.; GAUDEMET 1990, 454-455; FORNI 1992, 257 ff.; COARELLI 2001, 39-56.

29 Eus. v.C. 4,16; in Socr. h.e. 1,18,1 aber kommt es in einem Kontext vor, der mit dem Bericht über das Schicksal der „hellenischen“ Kulte und der gladiatorischen Spiele beginnt: દikóvas $\delta \grave{\varepsilon}$

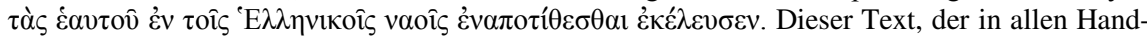
schriften enthalten ist, ist von Valesius unter dem Eindruck des Textes von Eusebios emendiert

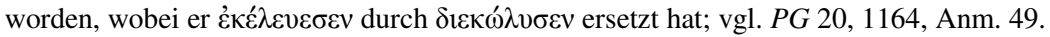

30 Eus. v.C. 2,48-60; vgl. BARNES 1981, 208 ff.; DE GiOvANNI 2003, 116.

31 Eus. v.C. 2,56,1-2 Diese Tatsache wird ausdrücklich von Libanios in der Schrift Pro templis, or.

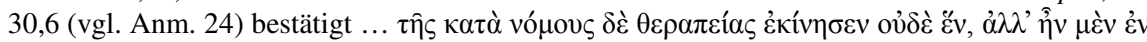

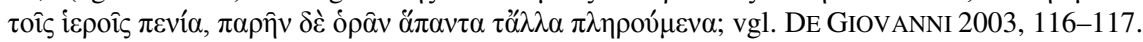


pelgüter einen theologischen und von der göttlichen Vorsehung bestimmten Beweis der Unfähigkeit der Götter, sich selbst zu verteidigen, ${ }^{32}$ dennoch enthält sein Text Informationen, die mit Eusebios' Zeugnis übereinstimmen. In einem Dokument aus den Jahren 343-350 beschreibt Firmicus Maternus eindringlich die vergoldeten und versilberten heidnischen Götzen. ${ }^{33}$ Nachdem er zweimal wiederholt hatte, dass die Götzenbilder aus Holz, Gold und Silber geschaffen sind, empfahl er den Kaisern ausdrücklich, diesen Reichtum für die Münzprägung und im allgemeinen zum Nutzen des Reichs zu verwenden:

Tollite securi, sacratissimi imperatores, ornamenta templorum. Deos istos aut monetae ignis aut metallorum coquat flamma, donaria universa ad utilitatem vestram dominiumque transferte (Firm. de err. prof. rel. 28,6).

Konkreter ist das Zeugnis von Hilarius, Bischof von Pictavi (Poitiers), das sich auf den Moment bezieht, in dem dem Bischof nach Aufhebung der Verbannung die Rückkehr aus dem Exil gestattet worden war, nachdem sich Julian gegen Constantius II. erhoben hatte. Er verfolgte aufmerksam die Entwicklung der kaiserlichen Religionspolitik und wusste über einen konkreten Punkt Bescheid: die Übertragung von Gütern, die aus heidnischen Tempeln stammten, auf die Kirchen. In einem Brief an Constantius II. gab der Bischof mit einer Genauigkeit, die in diesem Streitfall unerlässlich war, ${ }^{34}$ die Herkunft, die Rechtsgrundlage für die Einziehung und die Bestimmung dieser Güter an:

Auro rei publicae sanctum dei ornas et vel detracta templis vel publicata edictis vel exacto poenis deo ingeris. (Hilar. ep. ad Const. 1,6).

In Hilarius' Anklage werden alle grundlegenden verwaltungstechnischen Bedingungen genannt: Erstens geht die Schenkung an die Kirchen vom Kaiser aus und zweitens fallen die Tempelgüter im iter der Konfiskation unter die kaiserlichen Güter, und zwar gehören sie speziell zum Fonds der res privata.

Eine weitere Stimme, die diese Themen kritisch beleuchtet hat, wenn auch von einem anderen Standpunkt aus, ist die des anonymen Verfassers von De rebus bellicis, mit seiner Anklage einer malorum origo, die auf Konstantin zurückgehe (Constantini temporibus), da er die Tempel systematisch ,ihres Goldes, Silbers und ihrer Edelsteine" beraubt und diesen Reichtum zur Prägung von Goldmünzen verwendet hatte, die das soziale Gleichgewicht des Reichs in Mit-

32 Man bedenke die Aussagen von Firm. de err. prof. rel. 28,5: nihil possunt [scil. dii]. Et cum inciderit in domo deorum ligneorum et inauratorum et inargentatorum ignis, sacerdotes illorum liberabuntur, ipsi autem sicut trabes in medio comburentur. Regi autem et bello non resistent. Quomodo existimandum est vel recipiendum, quia sunt dii? Vgl. DE GIOvANNI 1980,143-144; BONAMENTE 1992, 190-191.

33 Vgl. TURCAN 1982, IV ff.; BARNARD 1990, 511.

34 BRENNECKE 1984, 361 ff.; MORESCHINI 1997, 110. Hilarius betrachtete auch das Vorgehen von Constantius II. zu Gunsten der Kirchen argwöhnisch; vgl. RosEN 1988, 63 ff. 
leidenschaft gezogen hatte. ${ }^{35}$ Das Kapitel, in dem der anonyme Verfasser diese umfassende Anklage formuliert, ist Gegenstand unterschiedlicher Interpretationen, bedingt auch durch die unsichere Datierung des Werks; ${ }^{36}$ dabei kann dem Leser der Widerspruch zwischen der konkreten Kritik an der spätantiken Gesellschaft einerseits wie an der Verschärfung des sozialen Ungleichgewichts, an der zunehmenden Prägung von Goldmünzen und an der Untragbarkeit der steuerlichen Belastung, ${ }^{37}$ und dem Gehalt des gesamten Kapitels (Ex quibus temporibus profusio vel avaritia coeperit) andererseits, der in einer moralistischen Verurteilung der Reformen Konstantins und dem Herbeisehnen des Goldenen Zeitalters besteht, entgehen.

In jedem Fall wird der Zusammenhang zwischen den konstantinischen Beschlagnahmungen zu Lasten der Tempel und der zunehmenden Prägung von Goldmünzen, die eine Reihe negativer Folgen hatte, deutlich:

Constantini temporibus profusa largitio aurum pro aere, quod antea magni pretii habebatur, vilibus commerciis assignavit; sed huius avaritiae origo hinc creditur emanasse. Cum enim antiquitus aurum argentumque et lapidum pretiosorum magna vis in templis reposita ad publicum pervenisset, cunctorum dandi habendique cupiditates accendit. (Anon. de rebus bell. 2,1-2).

Die Berichte von Eusebios und Firmicus Maternus über die Nutzung der Ressourcen, die den Tempeln entzogen wurden, finden Bestätigung und werden durch einige Einzelheiten ergänzt. ${ }^{38}$ In erster Linie konzentriert sich der anonyme Verfasser jedoch auf die negativen Konsequenzen in wirtschaftlicher und sozialer Hinsicht, die die plötzliche und massive Nutzung von Reichtümern hatte, die traditionell in templis repositae und nach dem Willen Konstantins ad publicum gebracht wurden. Diese Betrachtungen, in Verbindung mit der moralisierenden Anklage des Auftretens der cupiditas dandi habendique, machen auf die Vernachlässigung der traditionellen thesaurierenden Funktion der heidnischen Heiligtümer aufmerksam.

Erneut deutlich wird der Hauptzweck dieser systematischen Konfiskation: ad publicum pervenire, was die Folgerichtigkeit der Politik Konstantins bestätigt, der

35 Anon. de rebus bell. 2,4: Ex hac auri copia privatae potentium repletae domus in perniciem pauperum clariores effectae, teniuoribus videlicet violentia oppressis; vgl. MAZZARINO 1951a, 110 ff.; GIARDINA 1996, XXVII ff.; 51 ff. (einschließlich Bibliographie).

36 Anon. de rebus bell. 2,8-9: Denique paulisper felicium temporum revolve memoriam, et antiquae paupertatis famosa regna considera quae agros colere et abstinere opibus norant, qua haec honoris laude per omne aevum frugalitas incorrupta commendet. Certe aurea nuncupamus quae aurum penitus non habebant. Vgl. PASCHOUD 1976, 309 ff.; KOLB 1980, 518 ff.; BONAMENTE 1981, insbesondere 32 und Anm. 75; CALLU - MARANDON 1986, 588; LO CASCIO 1986, 551; BRANDT 1988, 39; BRANDT 1992, 213 ff. (insbesondere zur Bedeutung von vilia commercia); CATAUDELLA 1992, 284 ff.; 295 ff.

37 Anon. de rebus bell. 2,4 (zitiert oben Anm. 35).

38 Dem Paar aurum argentumque fügt der anonyme Verfasser eine große Menge Edelsteine hinzu, was zwar in keinem direkten Zusammenhang mit der Münzprägung stehen kann, aber dazu beiträgt, einen Eindruck von dem beachtlichen Umfang der Tempelgüter zu vermitteln. 
die Tempelgüter wegen ihrer Natur als öffentliches Eigentum für die publica utilitas bestimmte. ${ }^{39}$

Dass der letztendliche Bestimmungsort der Tempelgüter die christlichen Kirchen waren, lag im natürlichen Werdegang der neuen kaiserlichen Politik. Lapidar wird diese Tatsache im Chronicon Paschale verzeichnet, wo von einer direkten Übertragung von Tempelgütern auf die Kirchen im Jahre 325 berichtet wird: ${ }^{40}$

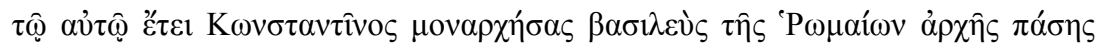

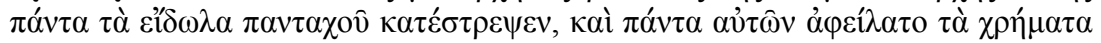

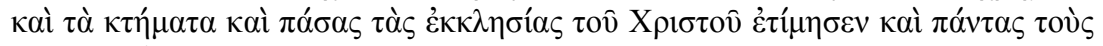

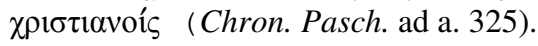

Die Berichterstattung im Chronicon kann zu dem falschen Glauben verleiten, dass eine direkte und sofortige Eigentumsübertragung von den Tempeln auf die Kirchen stattgefunden habe, ${ }^{41}$ weil sie sowohl den graduellen politischen Prozess (auch zum Schutze der öffentlichen Ordnung) verwischt als auch das komplexe verwaltungstechnische Verfahren übergeht und dabei nicht die vermittelnde Funktion der res privata berücksichtigt, die eine wichtige Rolle bei den Vorgängen spielte, an denen Dritte wie die Städte, andere Zweige der öffentlichen Verwaltung und oftmals auch Privatpersonen beteiligt waren.

Es ist in der Tat nicht wahr, dass die Kirchen die einzigen Empfänger der Spolien der heidnischen Tempel gewesen seien; ein beträchtlicher Teil kam dem Fiskus zu, der das Gold und Silber zur Münzprägung nutzte und dadurch die kaiserlichen Bilanzen aufbesserte (Konstantin war für seine Großzügigkeit gegenüber seinen Freunden bekannt); ${ }^{42}$ einige Güter sind nach der Konfiskation Eigentum der res publica geworden oder jedenfalls zur öffentlichen Nutzung bestimmt gewesen; ${ }^{43}$ und zuguterletzt darf das Kapitel der widerrechtlichen Aneignungen durch Privatpersonen und Beamte nicht vergessen werden. ${ }^{44}$

Auch in chronologischer Hinsicht muss die Datierung des Chronicon Pascha$l e$ als das Ergebnis einer zeitlichen Verkürzung interpretiert werden. Sie führt uns ins Jahr 325 zurück, in eine Phase, in der Konstantin im ganzen Reich seine

39 Vgl. Bodei GigLiONI 1977, 33-34.

40 Zusammen mit dem Sieg von Hadrianopolis und der Tötung des Crispus.

41 In zahlreichen sowohl christlichen als auch heidnischen Quellen wird die iunctura zwischen der Zerstörung der Tempel und dem Bau der Kirchen angeführt. Dazu zählen beispielsweise Jo. Mal. chron. 13,317 (im Zusammenhang mit einer Textstelle, an der von einer Vision des Kreuzes berichtet wird, nimmt diese Quelle Bezug auf die Zerstörung aller heidnischen Tempel und den Bau der Kirchen); Thphn. chron. a. m. 5810 (berichtet von der Übergabe heidnischer Tempel an die Christen). Auf der anderen Seite siehe Eun. vit. soph. 6,1,5.

$42 \mathrm{Zu}$ den gewöhnlichen Ausgaben für das Heer kamen für Konstantin die Ausgaben zum Bau Konstantinopels hinzu.

43 Zwei Beispiele aus Syrien und Germanien sind belegt; WREDE 1972, 8 (gehörte zu einem gallischen Tempel bei Trier); METZLER 1981, 37.

44 Zur Bereicherung von Beamten niedrigen Rangs, die von Ammianus beklagt wurde, vgl. Anm. 72. Zur Aneignung von Immobilien durch Privatpersonen, die Julian erfolglos zu bekämpfen versuchte, vgl. Anm. 67. 
Macht festigte. Für eine Operation dieser Art mögen in dieser Zeit die notwendigen Voraussetzungen bestanden haben, aber sie wird noch nicht ausgeführt worden sein. Hieronymus, der von einer subversio der Tempel im Jahre 331 berichtet, scheint glaubwürdiger zu sein, auch wegen des Zusammenhangs mit dem vorherigen Stichwort, das die dedicatio von Konstantinopel mit der Plünderung der Städte im ganzen Reich verbindet. ${ }^{45}$

Es fällt in der Tat die Vielfalt der Bestimmungen der Tempelgüter auf, wozu die Verwendung der Kunstwerke zur Verschönerung Konstantinopels, die Übertragung von Statuen auf öffentliche Baudenkmäler in Rom, die Nutzung des Edelmetalls zur Münzprägung und allgemein die Übertragung von Grundbesitz und der entsprechenden Einkommen auf die Kirchen, aber nicht nur auf sie, zählen. Es handelt sich dabei um mittelfristige Operationen, die sicherlich nicht nur in einem Jahr durchgeführt wurden, was die starken chronologischen Schwankungen zwischen den Quellen erklärt. Der Bau der neuen Hauptstadt am Bosporus zog sich über mehrere Jahre hin; ${ }^{46}$ auch die Umsetzung der kaiserlichen Entscheidungen in Rom, für die man als Bezugspunkt die Abschaffung des Amts des curator aedium sacrarum im Jahre 331 nehmen kann, ${ }^{47}$ war schrittweise; aus der Wiederaufnahme der Tätigkeit der Münzprägeanstalten kann man einen terminus ante quem erschließen, auch wenn sie nicht direkt auf die Nutzung des konfiszierten Metalls rückführbar ist. ${ }^{48}$ Was die öffentliche Finanzierung der ecclesiae betrifft, hatte sie bereits spätestens im Jahre 313 begonnen. ${ }^{49}$

Ein sicherer terminus ante quem ist die Tricennalienrede vom 25. Juli 336, der zufolge die Inventarisierung und Konfiskation bereits erfolgt war; ${ }^{50}$ ein wichtiger und stichhaltiger Bezugspunkt ist auch die Vermutung von Piganiol, die die Zustimmung von Chastagnol gefunden hat, wonach die Inventarisierung und Beschlagnahmung der Tempelgüter, wahrscheinlich parallel zu einer Klassifizierung der Tempelgebäude als res privata, auf das Jahr 331 zurückgehen und von Lucius Crepereius Madalianus, dem consularis aedium sacrarum für die Jahre 330 und 331, geleitet wurden. ${ }^{51}$

45 Hieron. chron. ad a. 331 (233b Helm): edicto Constantini gentilium templa subversa sunt (die Nachricht erscheint in ähnlicher Form in Oros. 7,28,28: edicto siquidem statuit citra ullam hominum caedem paganorum templa claudi); Hieron. chron. ad a. 331: dedicatur Constantinopolis paene urbium nuditate.

46 Um nur einige offizielle Anlässe zu nennen: offizielle Entscheidung am 8. November 324; inauguratio, Gewährung des ius Italicum und limitatio am 26. November 328; consecratio und dedicatio am 11. Mai 330; vgl. CRACCO RUGGINI 1979, $138 \mathrm{ff}$.

47 Seine Aufgaben wurden dem praefectus urbi anvertraut; vgl. CHASTAGNOL 1960, $45 \mathrm{ff}$.

48 Cracco Ruggini 1968, 776; Callu 1978, 115; Metzler 1981, 31-32; Callu 2003, 207.

49 LIZZI 2001a; vgl. oben Anm. 20 und unten Anm. 53.

50 Vgl. oben; insbesondere Anm. 2.

51 Piganiol 1974, 58-59; Chastagnol 1960, 52; BonAMENTE 1992, 175. Zeitgleich oder wenige Jahre zuvor wurden wesentlich drastischere Maßnahmen zum Schaden häretischer Sekten durchgeführt. Konstantin wies diese Güter direkt den katholischen Kirchen zu oder ließ sie versteigern; vgl. Eus. v. C. 3,65,3; DE GIOVANNI 2003, 94. 
Trotz gewisser unvermeidlicher Verbindungen müssen daher die Eingriffe, die seitens der Politik auf Kosten der Tempel für den Bau christlicher Kirchen erfolgten, in Rom wie im ganzen Reich klar getrennt werden. Hierbei lassen sich zwei Phasen voneinander unterscheiden: Die erste begann sofort nach dem Sieg an der Milvischen Brücke, die zweite nach dem Sieg über Licinius und der Erringung der Alleinherrschaft. In der ersten Phase, die mit dem Bau der Laterankirche und der Peterskirche die Stadt Rom direkt betraf, erscheint die Zuständigkeit der res privata vorrangig, wenn nicht sogar exklusiv, ${ }^{52}$ die auch die Kirchen der Diözese Africa unterstützte, wo der Donatistenstreit tobte. ${ }^{53}$ Nach dem Anschluss der östlichen Provinzen ging Konstantin mit größerer Entschlossenheit vor, wobei er auch Steuerbeamte mit einbezog und über die praefecti praetorio handelte. Diesbezüglich muss der Brief an die Bischöfe aus dem Jahre 324 erwähnt werden, mit dem sich diese über die praefecti praetorio die Gelder sicherten, die zur Restaurierung, Erweiterung oder zum Neubau von Kirchen notwendig waren. ${ }^{54}$ Nicht weniger bedeutsam sind die Anordnungen zum Bau der am 15. September 335 geweihten Grabeskirche, die dank eines Briefes an den Jerusalemer Bischof Makarios bekannt sind: Sie sahen die Mitwirkung des praefectus praetorio Orientis über seinen Stellvertreter Dracilianus und den consularis Palaestinae vor. ${ }^{55}$ Von besonderer Bedeutung war auch der Aus- und Umbau Konstantinopels zur kaiserlichen Hauptstadt, der die Nutzung umfangreicher finanzieller Ressourcen und die

52 Die gewöhnliche römische Verwaltung der opera publica, wozu die Tempel der offiziellen Kulte zählten, war durch die curatores und die Überschneidungen der Zuständigkeitsbereiche des Senats und des praefectus urbi sehr komplex. Vgl. KRAUTHEIMER 1993, 530; 537.

53 Der procurator rerum privatarum Heraclides trat bereits im Jahre 313 als Vermittler auf, als Konstantin dem Bischof von Karthago über den rationalis Africae Ursus 3000 folles schickte; vgl. Eus. h.e. 10,6,1-3. Bei einer weiteren Aktion des Kaisers zu Gunsten der Stadt CirtaConstantina wurde die Übertragung von Gütern der res privata auf die Kirchengemeinde ausdrücklich angeordnet; vgl. Optat. 213 (CSEL 38) (ad rationalem competentes litteras dedi ut domum bonorum nostrorum transgredi faciat cum omni iure suo ad dominium ecclesiae catholicae). Vgl. GiRARDET 1989, 194; KRAUTHEIMER 1993, 516 ff.; De Giovanni, $2001,61$.

54 Eus. v.C. 2,46,3: „Bei allen Kirchen also, denen du [Eusebios] entweder selbst vorstehst oder deren Vorsteher, soweit sie sich an anderen Orten befinden, du kennst, seien es Bischöfe, Priester oder Diakonen, sollst du mahnen, daß man auf ihren Bau alle Sorgfalt verwende und die bestehenden entweder wieder herstelle oder größer mache oder aber, wo die Not es heischt, ganz neue baue. Was hierzu notwendig ist, sollst du für dich selber und durch deine Vermittlung auch der übrige Episkopat von den Befehlshabern und von der Provinzstatthalterschaft verlangen; denn diesen ist der Befehl übersendet worden, mit aller Bereitwilligkeit den Worten deiner Heiligkeit nachzukommen."; vgl. KRAUTHEIMER 1993, $512 \mathrm{ff.}$

55 Eus. v.C. 3,30-40; insbesondere 31,2: „Was nun die Ausführung und die Ausschmückung der Mauern betrifft, so wisse, daß ich die Sorge dafür unserm Freunde Drakilian, der die Stelle der angesehenen Statthalter vertritt, und dem Provinzstatthalter übertragen habe. Denn es ist von meiner Frömmigkeit der Auftrag gegeben worden, daß Künstler und Handwerker und alles, was ihnen deine Einsicht als notwendig zum Bau eingibt, sofort durch ihre Fürsorge gesandt werde“; vgl. MACMulLEN 1959, 207 ff.; KRAUTHEIMER 1993, 514 ff. 
Wiederverwendung zahlreicher Kunstwerke aus dem ganzen Reich mit sich brachte. $^{56}$

Es wurde bereits angemerkt, dass sich Hilarius in einem Moment an den Kaiser wandte, als Konstantins Politik in den Hintergrund getreten war, da zu diesem Zeitpunkt bereits die zweite Phase der antiheidnischen Politik unter Constantius II. eine vollkommene Umsetzung gefunden hatte: Constantius II. hatte die SchlieBung der Tempel als Kultstätten angeordnet und ein generelles Opferverbot erlassen. ${ }^{57}$ Er bezog sich in der Tat nicht nur auf aurum, sondern auch auf andere bona templorum, bezüglich derer er die detractio, publicatio und exactio verfügte. ${ }^{58}$

Das Gesetz Cod. Theod. 16,10,4, dessen Datierung zwischen 346 und 356 schwankt, $^{59}$ schrieb außer dem Opferverbot ${ }^{60}$ die Schließung der Tempel vor, indem es den Zutritt zu Kultzwecken untersagte:

... placuit omnibus locis atque urbibus universis claudi protinus templa et accessu vetito omnibus licentiam delinquendi perditis abnegari. Volumus etiam cunctos sacrificiis abstineri... (Cod. Theod. 16,10,4).

In Abweichung von den Verfügungen Konstantins und parallel zu einer drastischen Verschärfung der antiheidnischen Politik, über die zeitgenössische Quellen eingehend berichten, verweigerte Constantius II. den Tempeln ihre religiöse Funktion und eröffnete die Phase der Veräußerung sowohl der unbeweglichen Tempelgüter als auch der Tempelgebäude selbst. ${ }^{61}$ Dieser Vorgang von mittelfri-

56 MANGo 1963, 53-75; DAGRON 1984, 128-132 (mit einer Liste der zu einem Großteil von Konstantin selbst nach Konstantinopel gebrachten Statuen mit Angabe ihrer Herkunft); KRAUTHEIMER 1993, 548 ff.

57 Es muss dabei berücksichtigt werden, dass für Gesetzesbrecher die Todesstrafe vorgesehen war; vgl. Cod. Theod. 16,10,4; 6; DE GiOvANNi 1980, $149 \mathrm{f}$.

58 Die antiken Quellen berichten von einer deutlichen Zäsur zwischen der Politik Konstantins, die durch die Verachtung und Aufgabe traditioneller Kulte gekennzeichnet war, und der von Constantius II., die darauf ausgerichtet war, die Kulte zu verbieten; vgl. Iul. c. Heracl. VII 22 b-c.; Rufin. h.e. 2,19. Vgl. CALDERONE 1968, 645; BARNES 1989b, 306 ff.; GAUDEMET 1990, 455; THÉlAMON 1990, 525 ff.; CALTABIANO 1991, 273; BONAMENTE 1992, 192; LEPPIN 1999, 466 ff.; $475 \mathrm{ff}$.

59 Die subscriptio datiert das Gesetz auf das Jahr 346 (der Codex Justinianus auf 356); in der neueren Forschung werden die Jahreszahlen 353, 354 oder 356 vertreten; vgl. NOETHLICHS 1971, 273-274; KLEIN 1977, 59 ff.; NOETLICHS 1986, 1167 ff.; PIETRI 1989, 113 ff.; GAUDEMET 1990, 449 ff.; KLEIN 1995, 131 ff.; CUNEO 1997, CI; 152; 308-10 (1. Dez. 356, zusammen mit Cod. Theod. 9,16,4 [Cod. Iust. 9,18,5] und Cod. Theod. 9,16,5 [Cod. Iust. 9,18,6]); DE GIOVANNI 2003, 138, $163 \mathrm{ff}$.

$60 \mathrm{Zu}$ den vorausgegangenen gesetzgeberischen Maßnahmen zählt Cod. Theod. 16,10,2, erlassen von Constantius II. im Jahre 341 (mit Bezug auf Konstantin, vgl. Anm. 5). Vgl. NoETHLICHS 1971, 3; 273 Anm. 384; SALZMANN 1990, 208-209; LEPPIN 1999, 468 ff.

61 Vgl. Anm. 24. In Alexandria führte die Agitation des Bischofs Georg zur Zerstörung von drei Kultstätten unter dem dux Aegypti Artemius: Er ließ das Sarapeion plündern, den Tempel des Genius verspotten und gab das Mithras-Heiligtum der Lächerlichkeit preis. In derselben Stadt ließ der praepositus monetae Dracontius einen Altar aus der Münzprägestätte entfernen. Vgl. Iul. ep. 60; Amm. 22,11,7; 9; Socr. h.e. 3,2; Soz. h.e. 5,7,4-7; DUMMER, 1971, 121 ff.; CALTABIANO 1985, 17 ff.; HAAS 1991, 281 ff.; LEPPIN 1999, 476; HAHN 2004, 68 ff. In Antiochia hat 
stiger Dauer, der auf rechtlicher Ebene seinen Höhepunkt im Jahre 407 mit dem Gesetz des Honorius zur öffentlichen Nutzung der aedificia ipsa templorum erreichte, findet im Codex Theodosianus keine kontinuierliche und detaillierte Erwähnung, vermutlich aus den beiden folgenden Gründen: Einerseits gingen viele Fälle der Plünderung und Zerstörung von Tempeln auf die Initiative organisierter christlicher Gemeinden oder Gruppen zurück, andererseits war die Mitwirkung der Provinzbehörden oft eher von Mitwisserschaft als von der Umsetzung der kaiserlichen Vorschriften bestimmt. ${ }^{62}$

Von Constantius II., der eine sehr entschlossene Religionspolitik verfolgte und die Schließung aller Tempel anordnete (mit dem bereits erwähnten Gesetz Cod. Theod. 16,10,4, das an den praefectus praetorio Taurus gesandt worden war), sind jedoch keine Gesetze bekannt, die ihre Zerstörung genehmigt oder gar gefördert hätten; ${ }^{63}$ es sind im Gegenteil in erster Linie constitutiones erhalten, wie jene an den praefectus urbi Aco Catullinus Philomathius, die sich gegen die Plünderung der Tempel der römischen Campagna richten: ${ }^{64}$

Quamvis omnis superstitio penitus eruenda sit, tamen volumus ut aedes templorum, quae extra muros sunt positae, intactae incorruptaeque consistant. (Cod. Theod. 16,10,3)

der Caesar Gallus den Apollon-Tempel von Daphne beseitigt und ein Märtyrergrab zu Ehren des Hl. Babylas eingerichtet; der Tyche-Tempel wurde geschändet; vgl. Lib. ep. 88; Iul. mis. 346b; Eutr. 6,14,2; Jo. Chrys. de S. Babyla 12-13; Socr. h.e. 3,18; Soz. h.e. 5,14; Thdt. h.e. 3,3; HAHN 2004, 131. In Arethusa wurde das wichtigste Heiligtum der Stadt vom Bischof Markos in eine christliche Kirche umgewandelt; vgl. Gr. Naz. or. 4,88; Thdt. h.e. 3,6,6-7; Soz. h.e. 5,4; 10; 11 (mit Datierung unter der Herrschaft Konstantins); DE Giovanni 1980, 142. In der Stadt Caesarea in Kappadokien wurden die Tempel von Zeus und Apollon abgerissen (dem TycheTempel blieb dieses Schicksal jedoch erspart); vgl. Soz. h.e. 5,4,1-2; Cassiod. hist. trip. 6,4. In Kyzikos hat der Bischof Eleusios einige Tempel geschändet; vgl. Gr. Naz. or. 4,88; Soz. h.e. 5,10,8; Thdt. h.e. 3,7,6-10. In Ilion blieb der Athena-Tempel unter dem Schutz des Bischofs Pegasios erhalten; vgl. Iul. ep. 79 (im Sommer 362 durfte Julian den Tempel mit Entgegenkommen des Bischofs besuchen, der anschließend vom christlichen Glauben abfiel); CALTABIANO 1991, 170; LEPPIN 1999, 478. In Didyma bei Milet wurde das Heiligtum des Apollon beschädigt; in der Stadt Meros in Phrygien wurde ein Tempel geschlossen; vgl. Soz. h.e. 5,20,7; Socr. h.e. 3,15; DEICHMANN 1939, 129, n.61; METZLER 1981, 30.

62 Außerdem muss berücksichtigt werden, dass die Auswahl der Gesetzestexte erfolgte, als die Konfiskation der Tempelgüter im Wesentlichen abgeschlossen war. Vgl. MACMULLEN 1984, 97; BONAMENTE 2007, 23.

63 Gregor von Nazianz behauptet dagegen, dass die Gesetze von Constantius II. die Zerstörung

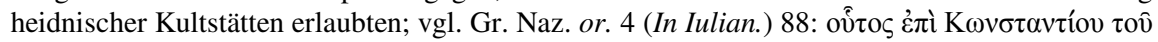

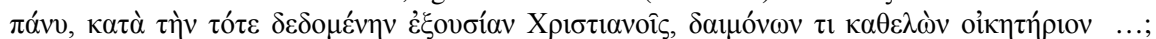

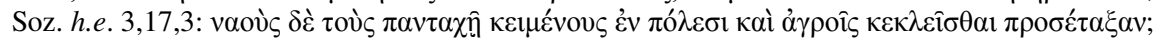
4,10,7: Thdt. h.e. 5,21,1-2 (oben Anm. 26).

64 Aco Catullinus Philomathius war Heide: Während seiner Amtszeit als praeses in Gallaecia ließ er ein Gebäude oder einen Altar zur Verehrung des Jupiter Optimus Maximus errichten, und es muss davon ausgegangen werden, dass er selbst Kaiser Constantius II. aufforderte, ihm im Jahre 342 das Edikt Cod. Theod. 16,10,3 auszustellen; vgl. FOWDEN 1978, 58 ff.; BARNES 1989b, 325 ff.; FRASCHETTI 1999, 107; LEPPIN 1999, 475 f.; LIZZI TESTA 2001, 694. 
Für die Stadt Rom, in der Constantius anlässlich seines Besuches im Jahre 357 die Beseitigung des Victoriaaltars aus der Kurie anordnete, ${ }^{65}$ sind vor allem Verfügungen zum Schutz der Tempel in architektonischer Hinsicht bekannt. Für den Zeitraum, in dem das Phänomen am stärksten verbreitet war, das heißt in der zweiten Hälfte des 4. Jahrhunderts, geben die gleichen Quellen, die die SchlieBung der Tempel und das Verbot der Kultausübung (unter Androhung schwerster Strafen) betonen, kaum Auskunft über die Bestimmung der Gebäude. Der hl. Martin, der 371 Bischof wurde, erlangte Ruhm als Tempelzerstörer in Gallien, ${ }^{66}$ Libanios beklagte um 385/386 in seiner Schrift Pro templis die Zerstörung der ländlichen Tempel und vor allem die Aneignung der Tempel durch einige hohe Beamte, die vom Kaiser selbst gedeckt wurden, ${ }^{67}$ jedoch finden diese Berichte keine Bestätigung in den erhaltenen gesetzlichen Bestimmungen. Bis zu Eugenius' Usurpation gab es zahlreiche Tempel sowohl in Rom selbst als auch im ganzen Reich. ${ }^{68}$

In den etwa 80 Jahren, die von der Alleinherrschaft Konstantins bis zu dem oben erwähnten Gesetz des Honorius vergingen, verloren die Tempel bis auf wenige Ausnahmen ihre religiöse und wirtschaftliche Funktion, und somit gingen die Auspizien, die in der Tricennalienrede Ausdruck fanden, in Erfüllung: die Übertragung der spolia von den besiegten Religionen auf die christlichen Gemeinden. Ein Zeitraum von weniger als einem Jahrhundert ist kurz, wenn man die Tiefe der historischen und kulturellen Wurzeln der Tempel berücksichtigt. Das Phänomen, das Gegenstand dieser Untersuchung ist, wurde nicht durch eine allmähliche Abwendung vom Heidentum hervorgerufen, sondern durch ein entschlossenes Vorgehen, wobei es nicht leicht ist, die Initiativen des Kaisers von denen der Bischöfe, Mönche und allgemein der christlichen Gemeinden zu unterscheiden. Die überlieferten Gesetze, die natürlich das Ergebnis einer Auswahl sind, die durch die Situation unter der Herrschaft von Theodosius II. geprägt wurde, beruhen auf einer Politik, die im Zeichen einer einheitlichen religio im Römi-

65 Vgl. Symm. rel. 3,3-7; Ambr. ep. 18,32; die Entscheidung von Constantius II. hatte einen hohen ideellen Wert; es muss jedoch berücksichtigt werden, dass der Kult weiter finanziert wurde und dass der Kaiser sogar dafür sorgte, die Priesterkollegien in Rom wieder herzustellen; Ammianus' Bericht von dem triumphalen adventus verwischt dieses Thema (Amm. 16,10). Es sind keine Zeugnisse über das Schicksal des Altars überliefert, der jedoch später wieder aufgestellt wurde, sowohl unter Julian als auch zur Zeit des Usurpators Eugenius; vgl. EDBROOKE 1976, 40-61; SAlZMAn 1990, 205 ff.; Klein 1979, 98 ff.; PASCHOUd 1990, 546; BonAmente 2000, 113-138; umstrittener war vor dem Hintergrund der besonderen geschichtlichen Umstände die zweite Entfernung des Altars, die von Gratian angeordnet wurde.

66 Sulp. Sev. v. Mart. 12-15; vgl. KLEIN 1995, 150f. LizZI im Druck.

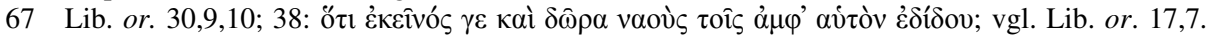
Auf die Aneignung der Tempel durch Privatpersonen nimmt auch die Verfügung Jovians (Cod. Theod. 10,1,8) Bezug (siehe unten). Zur Datierung der Rede vgl. PETIT 1951, 285 ff.

68 Vgl. Anm. 91. 
schen Reich die traditionellen Kulte zu einem schnellen und traumatischen Untergang verdammte. ${ }^{69}$

Die kaiserliche Gesetzgebung des 4. Jahrhunderts war dadurch gekennzeichnet, dass einerseits die heidnischen Kulte verboten wurden, man andererseits bemüht war, die Gebäude zu schützen, indem man gelegentlich auf ihren künstlerischen Wert oder auf ihre folkloristische Funktion hinwies. ${ }^{70}$ Diese Situation hat viel Freiraum für eigenmächtiges Handeln und Auseinandersetzungen zwischen Bischöfen und Mönchen einerseits und der kaiserlichen Gewalt andererseits gelassen - es sei nur an die harte Auseinandersetzung zwischen Theodosius I. und Ambrosius bezüglich der Synagoge von Kallinikon ${ }^{71}$ erinnert - aber im Allgemeinen hat sie dafür gesorgt, dass die Zerstörungen und Veräußerungen der Tempel uneinheitlich erfolgten, fast hat es den Anschein, dass sie auf einzelne lokale Initiativen oder Personen zurückgingen. Von Ammianus wissen wir, dass sich unter Julian auch kaiserliche Beamte niedrigen Ranges mit den Tempelgütern bereicherten ${ }^{72}$ während das Corpus agrimensorum das Phänomen der widerrechtlichen occupatio des Grundbesitzes der Tempel verzeichnet. ${ }^{73}$ Bei der Bewertung der Zeugnisse in Bezug auf die Zerstörung der Tempel und deren Umbau in christliche Kirchen muss die juristische Natur der Tempel selbst und der zugehörigen fundi als bonum publicum berücksichtigt werden, die es mit sich brachte, dass deren Veräußerung entweder vor- oder nachher von einer öffentlichen Behörde genehmigt werden musste, angefangen vom Kaiser selbst über die praefecti praetorio und deren Stellvertretern bis zu den Statthaltern der Provinzen. ${ }^{\text {.4 }}$

Zwischen Konstantin und Theodosius II. erfuhren Tempel Beschlagnahmungen, Plünderungen und Zerstörungen, aber auch Umwandlungen in Kirchen. Einige generelle Merkmale gehen aus den Quellen hervor: Die Initiative lag vorwiegend bei christlichen Gemeinden, die von Bischöfen, manchmal auch von Mönchen angeführt wurden; die öffentlichen Behörden konnte man der Mitwisserschaft, oft sogar der Mittäterschaft beschuldigen; selten blieben jedoch Eingriffe des Kaisers selbst, der im Gegenteil zuweilen Einschränkungen, Rückgaben

69 Man beachte die Überlegungen allgemeiner Art in GEFFCKEN 1929; HANSON 1980, 959 ff.; LANE FoX 1984, 574 ff.; CHUVIN 1990; BEATRICE 1990, $441 \mathrm{ff}$.

70 Ein Paradebeispiel für Schutzbestimmungen dieser Art ist Cod. Theod. 16,10,8, die im Jahre 382 von Theodosius an den dux Osdroenae Palladius geschickt wurde und vorsah, das in jener Provinz vorhandene Heiligtum für öffentliche Versammlungen zu öffnen, um den Besuchern die darin enthaltenen Kunstwerke zu zeigen. Gleichzeitig wurde jedoch betont, dass die Ausübung des Kultes verboten war.

71 Vgl. Anm. 102.

72 Amm. 22,4,3: Pasti enim ex his quidam (scil. Palatini spadones) templorum spoliis; vgl. Anm. 67.

73 Frontin. de controv. 13: Multi lucos profanos sive templorum loca occupaverunt et serunt (Agennius Urbicus).

74 Deichmann 1939, 105 ff. (Liste mit 89 Fällen); De Giovanni 1980, 142-143; NoethlichS 1986, 1149 ff. Zur Exorzierung der Tempelstätten im Falle der Nutzung für eine Kirche vgl. DeichmanN, s.v. Christianisierung, 1230 ff. und HahN 2000. 
und Restaurierungen anordnete. ${ }^{75}$ Im Falle des Bischofs Markos von Arethusa behauptete Gregor von Nazianz, er habe in Übereinstimmung mit den kaiserlichen Gesetzen gehandelt, während Sozomenus der Ansicht war, er habe gegen sie verstoßen. Es handelt sich um einen beispielhaften Streitfall, da die Aktionen gegen die Tempel in Übereinstimmung mit der ratio der kaiserlichen Religionspolitik und speziell mit den von Constantius II. erlassenen Anordnungen zur Schließung der Tempel und dem Opferverbot standen, die kaiserlichen Verfügungen jedoch nicht zur Zerstörung, Beschädigung und Plünderung der Tempel berechtigten. ${ }^{76}$ Dennoch wird deutlich, dass die Gesetze von Constantius II. zur Schließung der Tempel dem Bedürfnis, die funktionslos gewordenen heidnischen Kultgegenstände zu Gunsten der christlichen Gemeinden wiederzuverwerten, Nachdruck verliehen.

Wie bereits festgestellt wurde, war nach Hilarius das Medium zur Übertragung der Tempelgüter die res privata, deren gewöhnliche Einnahmequelle aus den bona damnatorum bestand. Über diesen Zweig der kaiserlichen Vermögensverwaltung verfolgte umgekehrt Julian Apostata seine Restaurationspolitik hinsichtlich der städtischen Finanzen und der Tempelgüter: Die Wiedereröffnung der Tempel und die Wiederherstellung der finanziellen Autonomie der Städte wurden in der Tat über die res privata geregelt. ${ }^{77}$

Auch wenn ein wesentliches Ziel der Politik Julians in der Übertragung des Grundbesitzes der res privata auf die Städte bestand, hatte dieses Programm viele Berührungspunkte mit den Tempelgütern, die bereits von derselben kaiserlichen Verwaltung eingezogen worden waren. In seiner Politik bildeten sich einige kritische Punkte heraus, die im Epitaphios, der von Libanios für Julian verfasst wurde, genau angegeben sind; sie bestanden im Wiederaufbau und der Restaurierung der Tempel sowie der erneuten Aufstellung der Statuen an ihren alten Standorten, in Verbindung mit der Gewährung von Vergünstigungen gegenüber den Städten für die Umsetzung dieser Programme. ${ }^{78}$ Im Gegenzug führte Julian Strafgelder für die Personen ein, die den Tempeln Baumaterial entwendet hatten, ließ einige Kirchen abreißen und widerrechtlich beschafftes Eigentum konfiszieren. ${ }^{79}$ Es handelt sich um eine Reihe von Verfügungen, die von der Geschichtsschreibung und den Rechtscodices vernachlässigt worden sind. Nur der Verfasser der Histo-

75 Julian Apostata hat von Bischöfen wie Eleusios und Beamten wie Artemios Rechenschaft für die von ihnen verursachten Schäden gefordert; vgl. Amm. 22, 11, 2 (Artemios' Verurteilung zum Tode); Soz. h.e. 5,10,9; Thdt. h.e. 3,18,1; MARASCO 1997, $59 \mathrm{ff}$.

76 Gr. Naz. or. 4,88-91; LEPPIN 1999, 476; 477 Anm. 108.

77 Vgl. CONDURACHI 1941, 119 ff.; BONAMENTE, 1983, 54 ff.; PACK 1986, 143-153.

78 Lib. or. 18 (Epitaphios), 126; 129. Unter den zahlreichen Fällen sei an Emesa mit seinem Helios-Tempel (Iul. or. 12,357 c-d), Gaza (Soz. h.e. 5,3,4,), Hierapolis (Iul. ep. 98,401 b-d) und Apamea erinnert. Vgl. dazu ARCE 1975, 201 ff.; ATHANASSIADI FOWDEN 1981, 124.

79 Lib. or. 18 (Epitaphios), 126. Im XV. Buch De operibus publicis des Codex Theodosianus könnte das Gesetz 15,1,3 (das Restaurierungen und dem Tempelbau Vorrang verleiht) Julian zugeschrieben werden, obwohl inscriptio und subscriptio auf Konstantin Bezug nehmen; vgl. DEICHMANN 1939, 106; ARCE 1975, 201-215. 
ria acephala nennt ein Edikt, das am 4. Februar 362 in Alexandria erlassen wurde und das die Rückgabe von Statuen und Gütern vorsah, die aus heidnischen Tempeln entwendet worden waren. ${ }^{80}$ Libanios berichtet außerdem über einige Fälle von Wiederinstandsetzungen von Tempeln, die zuvor von Privatpersonen erworben worden waren. ${ }^{81}$ Es gab jedoch auch Übergriffe und Zerstörungen heidnischer Tempel, von denen einige Reaktionen auf die kaiserliche Politik darstellten. $^{82}$

Besser dokumentiert sind die gegensätzlich lautenden Vorschriften seiner Nachfolger Jovian, Valentinian I. und Valens, die darauf ausgerichtet waren, sämtliche Tempelgüter der res privata zurückzuerstatten. Die Reaktionen auf die Tempelpolitik Julians waren nicht so sehr politischer und religiöser Art, sondern nahmen in erster Linie Bezug auf die komplizierten Rechtsbeziehungen zwischen Städten, Kirchen und Privatpersonen, die auf verschiedene Art und Weise in den Besitz von Tempelgut gelangt waren. Die erste Verfügung wurde am 4. Februar 364 von Mailand aus an den comes rei privatae Caesarius gerichtet: ${ }^{83}$

Universa loca vel praedia, quae nunc in iure templorum sunt quaeque a diversis principibus vendita vel donata sunt retracta, ei patrimonio, quod privatum nostrum est, placuit adgregari. (Cod. Theod. 10,1,8).

Diese Vorschrift wurde ausdrücklich durch eine zweite vom 23. Dezember desselben Jahres an den praefectus praetorio Mamertinus bestätigt, die nicht ohne polemischen Ton deutlich macht, dass die betreffenden Güter bereits zuvor zum Privatvermögen des Kaisers gehört hatten:

80 Hist. aceph. 3,1 (Martin); vgl. Amm. 22,5,2: [Iulianus] planis absolutisque decretis, aperire templa arisque hostias admovere, et deorum restituire statuit cultum; BOWERSOCK 1980, 123124; ARINA 1985, 197-239; BONAMENTE 1986, 159-163.

81 Es handelt sich um einen Fall in Antiochia (Lib. ep. 724), einen in Tyros (ep. 828) und einen, der sogar den praefectus praetorio Thalassios betraf (Lib. ep. 1364); vgl. BARNES 1989b, 328 ff.; HAHN 2004, 131-132.

82 SieVERS 1868, 103; PIGANIOL 1974, 155 ff.; JONES 1972, I, 165. In Rom wurde der dem Apollo Palatinus geweihte Tempel am 18. März 363 in Brand gesteckt, in Antiochia wurde der ApollTempel von Daphne angezündet; vgl. Sozom. h.e. 5,4,1-2. In Arethusa zerstörte der Bischof Markos 362 eine heidnische Kultstätte, um an deren Stelle eine Kirche zu errichten. Er wurde anschließend von Heiden ermordet; vgl. Soz. h.e. 5,10,8-14; Thdt. h.e. 3,7,6-10; DEICHMANN 1939, Anm. 5. In der Stadt Caesarea wurde der Tyche-Tempel zerstört; vgl. Soz. h.e. 5,4,1-2. In Gaza gab es einen christlichen Übergriff auf einen Tempel; vgl. Soz. h.e. 5,9; HAHN 2004, 196197 (zum Konflikt, der durch die Spannungen zwischen Gaza und seinem Hafenort Maiuma erschwert wurde). In Kyzikos wurde der Bischof Eleusios beschuldigt, eine Kapelle der Novatianer zerstört und Übergriffe auf die Heiden gefördert zu haben; vgl. Soz. h.e. 5,15,5. Es ist die Nachricht von der Zerstörung eines Altars in Durostorum (Chron. Pasch. 549) und eines in Phrygien im Zuge einer durch Macedonius angestifteten Hetzerei überliefert (Soz. h.e. 5,10). Beschädigungen von Statuen wurden aus Meros in Phrygien gemeldet (Socr. h.e. 3,15. Soz. h.e. 5,11: es handelte sich um einen kurz zuvor wieder aufgebauten Tempel; die christlichen Täter wurden dafür getötet).

83 Das Gesetz Cod. Theod. 10,1,8 wird Valentinian und Valens zugeschrieben, aber seine Datierung (4. Februar 364) deutet daraufhin, dass es bereits von Jovian erlassen worden sein muß; vgl. Socr. h.e. 3,24; PIGANIOL 1974, 166; HAHN 2004, 178. 
Universa, quae ex patrimonio nostro per arbitrium divae memoriae Iuliani in possessionem sunt translata templorum, sollicitudine sinceritatis tuae cum omni iure ad rem privatam nostram redire mandamus. ${ }^{84}(\mathrm{Cod}$. Theod. 5,13,3).

Julian hatte jedenfalls festgestellt, dass die Tempel umfangreiche Finanzierungen benötigten, um ihre Funktionen wieder aufzunehmen und sogar mit Unterstützung des Kaisers die Konkurrenz der Kirchengemeinden nicht nur in der Kultausübung, sondern auch in der sozialen Fürsorge zu überstehen. ${ }^{85}$

Bedeutsam für den juristischen Status der Tempel unter Valentinian I. und Valens mit direktem Bezug auf die Stadt Rom ist ein Edikt von Valentinian I., das im Jahre 364 in Mailand an den praefectus urbi Symmachus erlassen wurde und vorschrieb, zur Bewachung der heidnischen Tempel keine Männer christlicher Religion zuzulassen (Cod. Theod. 16,1,1: ...Quisquis seu iudex seu apparitor ad custodiam templorum homines Christianae religionis adposuerit sciat non saluti suae, non fortunis esse parcendum... $){ }^{86}$ Das Gesetz sah den Fortbestand und die Benutzbarkeit der Tempel vor und bestimmte, dass ihre Kontrolle eine öffentliche Angelegenheit war. Wenn man die hervorgehobene Position des Edikts im Codex Theodosianus, den Namen des Empfängers, der ein herausragender Vertreter der heidnischen Tradition war, und speziell die Tatsache bedenkt, dass der praefectus urbi die Kompetenzen wahrnahm, die zuvor zum Zuständigkeitsbereich des curator aedium sacrarum gehört hatten, muss man davon ausgehen, dass das Gesetz nicht die aeditui (Diener der sacerdotes, verantwortlich für die Öffnung und Vermögensverwaltung der Tempel) betraf. Es wurden damit die Zuständigkeiten der iudices mit ihren apparitores geregelt, etwa die Überwachung der Tempel unter sicherheitstechnischem Aspekt, womit Soldaten beauftragt waren. ${ }^{87}$

Zum Schutz der Kunstwerke der Tempel diente ein Erlass vom November 382 an den dux Osdroenae Palladius, in dem der Zutritt zum Tempel von Edessa genehmigt wurde, um die Kunstwerke im Tempel zu besichtigen. ${ }^{88}$

Vom Tode Julians bis zur Thronbesteigung Theodosius' I. ließ sich keine Verschärfung der Gesetzgebung verzeichnen - dieser Zeitraum wurde im Gegenteil durch Anzeichen von Kontinuität oder Wiederaufnahme der traditionellen Kulte geprägt; die von Valentinian I. am Anfang seiner Herrschaft geäußerte Absicht, die kaiserliche Regierung über die religiösen Konflikte zu stellen und die verschiedenen Formen des Kultes im Reich zu tolerieren, wurde grundsätzlich

84 MASI 1971, 28; GAUDEMET 1990, 456. Bemerkenswert ist, dass die Zuteilung von fundi aus der res privata unter dem Rechtstitel der possessio erfolgte.

85 Julian hat die Intoleranz der Christen und die Widersprüchlichkeit ihrer Politik in Bezug auf die Lehren des Evangeliums in aller Deutlichkeit aufgezeigt: Iul. adv. Galil. 205e-206a.

86 Vgl. MazZarino 1974a, 420; Cracco RugGini 1979, 95, Anm. 287.

87 DE GIOVANNI 1980, 27-31 (mit zeitgenössischen Beispielen).

88 Cod. Theod. 16,10,8; vgl. Noethlichs 1971, 327; De Giovanni 1980, 144-145, Anm. 109. Dieses Thema zum Schutz der Tempel kehrt auch in nachfolgenden Epochen wieder: 458 wies Maiorianus den praefectus urbi an, künstlerisch wertvolle Baudenkmäler, wozu die Tempel zählen, zu schützen (Nov. Maior. 4,1). 
beibehalten. ${ }^{89}$ Es wurden einige Verfügungen zum Schutze der heidnischen Kulte erlassen, ${ }^{90}$ und es wurden Voraussetzungen für eine Wiederaufnahme traditioneller Kulte geschaffen, vor allem in Rom. Im römischen Senat gab es noch ein Gleichgewicht zwischen traditionalistisch eingestellten Familien und denjenigen, die zum Christentum übergetreten waren. Zahlreiche Vertreter der erstgenannten Gruppe wurden praefecti urbi, ließen die heidnischen Tempel restaurieren und sorgten für die Wiederaufnahme traditioneller Kulte. Dieser Fortbestand der heidnischen Kulte, der oft als ,heidnische Renaissance“ bezeichnet wird, dauerte unter der Führung angesehener Senatoren wie Vettius Agorius Praetextatus, C. Ceionius Rufius Volusianus Lampadius, Quintus Aurelius Symmachus und Virius Nicomachus Flavianus fast ein halbes Jahrhundert an und fand ein tragisches Ende im Jahr 394 mit der Schlacht am Frigidus. ${ }^{91}$

Dennoch überwogen auch zu dieser Zeit in der Stadt Rom die Bedeutung und Anzahl der Neubauten zugunsten des Christentums, gefördert von der kaiserlichen und privaten Freigebigkeit als auch von der römischen Kirche selbst, vor

89 In einem Gesetz des Jahres 371 wurde an einen programmatischen Grundsatz erinnert, der in den ersten Regierungserklärungen zum Ausdruck kam; Cod. Theod. 9,16,9: Testes sunt leges a me in exordio imperii mei datae, quibus unicuique, quod animo inbibisset, colendi libera facultas tributa est. Ammianus gibt zu, dass der Kaiser diesen Grundsatz befolgt habe; vgl. Amm. 30,9,5: hoc moderamine principatus inclaruit quod inter religionum diversitates medius stetit ... nec interdictis minacibus, subiectorum cervicem ad id, quod ipse coluit, inclinabat, se intemeratas reliquit has partes ut repperit; LIZZI TESTA 2004, 251-252.

90 Die Bestimmungen, die der Restaurierung und Fertigstellung bestehender Gebäude gegenüber dem Abriss und dem Neubau Vorrang gaben, spiegelten eine traditionell geprägte Politik wider. Cod. Iust. 1,9,4, aus dem Jahr 370 verbot die Zerstörung der Synagogen; zwei Bestimmungen aus den Jahren 364 und 371 erkannten die Vorrechte der für den Provinzialkult zuständigen Priester an (Cod. Theod. 12,1,60; 75). Zur Verwendung von Baumaterialien aus bestehenden Gebäuden vgl. RUGGINI 1995, 81, Anm. 209-210. In diesem Zusammenhang sei daran erinnert, dass in dem Gesetz Cod. Theod. 16,2,18, das am 17. Februar 370 in Trier erlassen wurde, zum ersten Mal der juristische Fachbegriff paganus erscheint.

91 Der praefectus urbi Memmius Vitrasius Orfitus ließ eine dem Apollo gewidmete Ädikula errichten (CIL VI 45) und die privaten Gebäude, die zu dicht an den Kultstätten standen, abreißen (Amm. 27,9,10: discrevit ab aedibus sacris privatorum parietes, eisdem inverecunde coniectos); Vettius Agorius Praetextatus, der das gleiche Amt bekleidete, ließ 367-368 die Porticus deorum Consentium restaurieren (CIL VI, $102=I L S$ 4003: [Deorum Co]nsentium sacrosancta simulacra cum omni lo[ci totius adornatio]ne, cultu in f[ormam antiquam restituto / V]ettius Praetextatus vir clarissimus pra[efectus u]rbi [reposuit], / curante Longeio ... [ viro clarissimo c] onsul[ari operum publicorum]); Claudius Hermogenianus Caesarius, ein weiterer praefectus urbi, ließ 374 ,mit öffentlichen Geldern“ verschiedene Gebäude restaurieren, darunter die Porticus des Tempels des Bonus Eventus (Amm. 29,6,19; CIL VI 499). Wenige Jahre später ließ der praefectus annonae Sempronius Faustus den Isis-Tempel in Portus restaurieren (CHASTAGNOL 1969, 135 ff.). In der Relatio 21 aus dem Jahre 384 beklagte Symmachus (= Symm. rel.) die Plünderung heidnischer Kultstätten; vgl. VERA 1981, 366-367; 408 ff. Über diese „heidnische Renaissance" und die Wiederaufnahme des Studiums klassischer Autoren, siehe MAZZARINO 1951b, 121 ff. (mit Bezügen auf die grundlegende Arbeit von A. Alföldi über die Kontorniaten); Bloch 1963, 193 ff.; Pietri 1997, I, 81 ff.; FraschetTi 1999, 296 ff.; MitTaG 1999; LizZi TESTA 2001, $701 \mathrm{ff}$. 
allem unter Papst Damasus I. ${ }^{92}$ Auch wurden Eingriffe zum Schaden heidnischer Kultstätten verzeichnet, wie beispielsweise die Schließung eines MithrasHeiligtums mit Zerstörung der Kultstatuen, angeordnet vom praefectus urbi Furius Maecius Gracchus in den Jahren 376-377..$^{93}$

Die Kompilatoren des Codex Theodosianus bezogen sich auf die constituta divi Gratiani als Präzedenzfall der Einziehung aller den heidnischen Kulten zugehörigen Immobilien zu Gunsten der res privata, die 415 von Honorius und Theodosius II. angeordnet wurde und auf die nachfolgend eingegangen wird. ${ }^{94}$ Die constituta divi Gratiani selbst sind uns jedoch nicht überliefert. Von der Religionspolitik von Gratian sind wegen der Resonanz, auf die sie bei seinen Zeitgenossen gestoßen war, am besten die im Jahr 382 erlassenen Verfügungen zur erneuten Beseitigung des Victoriaaltars aus der Kurie bekannt, weswegen sich eine heftige Auseinandersetzung zwischen dem römischen Senat und dem mailändischen Hof entwickelt hatte. Die Hauptrollen in diesem Fall spielten Quintus Aurelius Symmachus, dem es 384, nach einem vergeblichen Versuch zwei Jahre zuvor, gelungen war, am Hofe von Mailand empfangen zu werden und in seiner Funktion als praefectus urbi die berühmte Relatio III de ara Victoriae vorzulegen, und auf der Gegenseite der Bischof Ambrosius, der seine Beschwerden vor Valentinian II. vorbrachte und diesen überzeugen konnte, die Politik seines Vaters zu bestätigen. ${ }^{95}$

Wir verfügen über Zeugnisse, die zusammenhängende und genaue Informationen über die wirtschaftlichen und steuerlichen Komplikationen der Politik Gratians zum Schaden der traditionellen Kulte, speziell der Vesta, enthalten. Gratian stellte die Finanzierung des Kultes durch öffentliche Gelder ein, ${ }^{96}$ trieb für das aerarium die annonae ein, die zuvor dem Kollegium der Vestalinnen über die

92 Vgl. PIETRI 1976, 461-462; CRACCO RUgGini 1982, 78 ff.; GUYON 1993, 56 ff.; PANI ERMINI SinISCALCO 2000; LIZZI TESTA 2004, 93-120.

93 Hieron. ep. 107,2; Prud. c. Symm. 1,561-5; vgl. LizZI TESTA 2001, 698 (vgl. ebd. den historischen Kontext und die Bibliographie). Auf diesen Zeitraum kann die Überlappung des Kultes von Exuperantius und Sabinus im Äskulap-Heiligtum auf der Tiberinsel zurückgehen; vgl. LIZZI TESTA 2004, 420 Anm. 337. In Antiochia wurde der Trajan-Tempel in Brand gesteckt und der Ares-Tempel wurde in den Umbau des Forums zu Ehren von Kaiser Valens miteinbezogen; auch die Grabstätte der Makkabäer wurde in eine Kirche verwandelt; vgl. Jo. Chrys. hom. 1-3 (PG 50, 617-628); Jo. Ant. fr. 273,2 Roberto (p. 460); Suid. I, 401, 'Iobianós (II 638,25-639,18 Adler); Downey 1961, 403 ff.; Hahn 2004, 179-184.

94 Es handelt sich um Cod. Theod. 16,10,20 aus dem Jahre 415, siehe dazu unten und Anm. 123.

95 Ambros. ep. 17,3-4; ep. 18,3; 14; Symm. rel. 3,8,11-13; dazu VeRA 1981, 12-23; 37-47. Vgl. auch Ambr. obit. Valent. 19-20; ep. 57 (an Eugenius); PALANQUE 1933, 117 f.; MAZZARINO 1974b, 339 ff.; VeRA 1981, 12 ff.; PASCHOUd 1983, 197 ff.; PASCHOUd 1986; ROSEN 1994, 29 ff.

96 Symm. rel. 3,7 (parens ille clementiae vestrae Romanis caerimoniis non negavit impensas); Ambr. ep. 17,3 (Maur.): miror quomodo aliquibus in spem venerit, quod debeas aras diis gentium tuo instaurare praecepto, ad usus quoque sacrificiorum profanorum praebere sumptum. Quod enim iam dudum vel fisco vel arcae est vindicatum, de tuo magis conferre videbere quam de suo reddere. 
arca frumentaria entrichtet wurden und führte davon einen Teil zu Gunsten der Zunft der baiuli ab. ${ }^{97}$ Was die Grundstücke betrifft, die zum Eigentum der Tempel und der Priesterkollegien gehörten, wies er sie der res privata zu und untersagte gleichzeitig Privatpersonen, Testamente zu Gunsten der Tempel zu verfassen. ${ }^{98}$ Damit schränkte Gratian die Privilegien und Zuwendungen zu Gunsten der Vestalinnen entschiedener ein als Constantius II. wenige Jahrzehnte zuvor. ${ }^{99}$

Für Theodosius I. hatte die Unnachgiebigkeit, mit der er gegen die traditionellen Kulte vorging, eine weitere Grundlage in der Verkündigung des nicänischen Christentums als einziger Staatsreligion des Reichs, wie im Edikt von Thessaloniki vom 28. Februar 380 klar festgelegt wurde. ${ }^{100}$ Diese neue Situation spiegelt sich bei Libanios wider, der etwa 390 in einer Rede bedauerte, dass die Tempelgüter mittlerweile in fremden Händen seien und auf den Altaren durch feste Immobilienrenditen finanzierte Opfergaben fehlten. In Pro templis berichtet er, dass die Tempel zwar noch zugänglich seien, beklagt aber das Opferverbot. ${ }^{101}$ Es sind Maßnahmen gegen Heiden, Häretiker und Juden bezeugt, die oft auf Druck der Bischöfe getroffen wurden (der Fall der Synagoge und der Kirche der Valentinianer in Nikephorion-Kallinikon ist beispielhaft) ${ }_{102}$ und durch die der doktrinären Unnachgiebigkeit der Nicäner Ausdruck verliehen wurde; und das alles im Rahmen einer Verschärfung der antiheidnischen Gesetzgebung, wie Cod. Theod.

97 Symm. rel. 3,15: ad mercedem vilium baiulorum sacra castitatis alimenta verterunt; vgl. CHASTAGNOL 1960, 158.

98 Symm. rel. 3,13: agros etiam virginibus et ministris deficientium voluntate legatos fiscus retentat. Vom Verbot ausgenommen waren die Schenkungen, die nicht in Form von Grundbesitz erfolgten, worauf Ambrosius hinwies: Ambr. ep. 18,16: sola sublata sunt praedia... praedia igitur intercepta, non iura sunt.

99 Gesamtbewertungen der Entscheidungen Gratians in VERA 1981, 46-47; PASCHOUD 1990, 564; LIZZI 1998, 103-106; 113-132.

100 Cod. Theod. 16,1,2; Cod. Iust. 1,1,1; vgl. EnssLin 1953; GAudEMET 1986, 1-22. Dazu, dass Justinian dieses Gesetz im Corpus Iuris Civilis an die erste Stelle setzen ließ, vgl. IRMSCHER 1990, 582-583; ERRINGTON 1997, 412.

101 Diesbezüglich Lib. or. 2,30 (etwa $380 \mathrm{n}$. Chr.) und 30,8 (zur Datierung zwischen 385 und 390, vgl. ROMANO 1982, 17 ff.; nach dem Jahr 388, da nach WIEMER 1995, 128 die Rede an den praefectus praetorio Tatianus gerichtet war). In Antiochia blieben zu dem Zeitpunkt, als Libanios die Rede Pro templis schrieb, die Tempel der Fortuna, des Zeus, der Athene und des Dionysos sowie die Tempel, die Hermes, Pan, Demeter, Ares, Kalliope, Apoll und Zeus geweiht waren, unversehrt; vgl. Lib. or. 15,79; 30,51; LIEBESCHUETZ 1972, 238; HAHN 2006, Anm.19.

102 Zur speziellen Stellung der Juden, die zumindest rechtlich anerkannt und geschützt waren, vgl. Cod. Theod. 16,8,9 (29. Sept. 393): Indaeorum sectam nulla lege prohibitam satis constat...; Cod. Theod. 16,8,25 (15. Feb. 423): Placet in posterum nullas omnino synagogas Iudaeorum vel auferri passim vel flammis exuri. Den Fall von Kallinikon unterstellte der comes Orientis Tatianus der direkten Zuständigkeit des Kaisers, der anordnete, die Synagoge auf Kosten der christlichen Gemeinde und des Bischofs, die zusammen mit den Mönchen für die Tat verantwortlich waren, wieder aufzubauen. Wie bekannt führte das entschiedene Vorgehen von Ambrosius dazu, dass die Entscheidung des Kaisers wirkungslos blieb. Vgl. SIMON 1964, 126 ff.; PAVAN 1966, 475 ff.; VOGLER 1979, 34 ff.; BlANCHETIÈRE 1981, 125 ff.; DE GIOVANNI 1980, 89; 111; 117 ff. Vgl. GOTTER in diesem Band, der im betreffenden Fall auf die Abstufung von einem Problem öffentlicher Ordnung zu einem Problem der religiösen Lehre hinweist. 
16,10,9 aus dem Jahr 385 (gegen die Wahrsagerei) oder Cod. Theod. 16,10,10 vom 24. Februar 391 (unter dem direkten Einfluss von Ambrosius in Mailand erlassen) belegen, letzteres eine Vorschrift, die das Opferverbot weiter verschärft, mit besonderem Bezug auf Magistrate und Beamte. ${ }^{103}$

Die Vorgehensweise von Cynegius, praefectus praetorio Orientis in den Jahren 384-388, liefert ein Beispiel für die Umsetzung der kaiserlichen Politik. ${ }^{104}$ Die Zusammenarbeit zwischen dem praefectus praetorio und dem Bischof von Alexandria, Theophilos, schuf die Voraussetzungen für Beschädigungen und Zerstörungen heidnischer Tempel auch ohne ausdrückliche Genehmigungen des Kaisers. Eine derartige Situation, die durch Plünderungen und Aneignungen der Tempelgebäude gekennzeichnet war, begünstigte direkt die Christen von Alexandria, insbesondere deren Bischof, der in diesen Jahren besonders reich und mächtig zu sein schien. Die systematische Unterdrückung der heidnischen Kulte erreichte mit der Zerstörung des Sarapeions in den ersten Monaten 392 ihren Höhepunkt. ${ }^{105}$

103 Cod. Theod. 16,10,10-11. Kirchenhistoriker weisen darauf hin, dass Theodosius der antiheidnischen Politik einen starken Impuls verlieh und sich von der Politik Valentinians klar abgrenzte; vgl. Rufin. h.e. 9,19; Thdt. h.e. 5,21,3-5. Siehe dazu PigANIOL 1974, 284: « La loi du 24 février 391 est une sentence de mort portée contre le paganisme »; GAUDEMET 1990, 458 ff. (mit einer zusammenhängenden Übersicht über die einzelnen Bereiche, in denen gegen die Heiden vorgegangen wurde). Was die Tempelzerstörungen betrifft, sind außer in Alexandria und der Gegend, in der die Übergriffe von Schenute (Anm. 104) stattfanden, die des Zeus-Tempels und zahlreicher anderer in der Stadt Apamea bekannt (der Bischof Marcellus verlor sein Leben in dem Aufstand, der durch die Zerstörung eines großen Tempels hervorgerufen wurde); vgl. Lib. ep. 1053 (11,177-9 Förster); Thdt. h.e. 5,21,5 ff.; Soz. h.e. 7,15,13-15; Deichmann 1939, Anm. 3; HAHN 2004, 285, Anm. 38; TesTA 1991, 314. In Damaskus wurde der Zeus-Tempel durch die Johanneskirche ersetzt; vgl. Chron. Pasch. 1,156; Deichmann 1939, Anm. 11. In Heliopolis wurde eine Basilika auf dem Grundstück des Großen Tempels errichtet; vgl. Chron. Pasch. 1,561; DeICHMANN 1939, Anm. 6. In der Präfektur Gallien erfolgte eine systematische Zerstörung von Tempeln in Nordgallien unter Leitung von Martin von Tours und Victoricius von Rouen; vgl. Sulp. Sev. v. Mart. 13, 14-15; Paulin. ep. 18, 4; FREND 1990, 480. In Eleusis wurde den Mysterienkulten ein Ende gesetzt; vgl. Eun. vit. soph. 7,3,1-5.

104 Das Gesetz Cod. Theod. 16, 10, 9 war an Cynegius adressiert (25. Mai 385: es enthielt ein strenges Opfer- und Wahrsageverbot). Maternus Cynegius zerstörte in den Jahren 385-388 zahlreiche Tempel; vgl. Rufin. h.e. 11, 23; Thdt. h.e. 5,21,5 ff.; Zos. 4,37,3; FOWDEN 1978, 53 ff. Ebenfalls in Ägypten plünderten für eine lange Zeit (von 383 bis 466) Mönchsbanden unter Führung des Mönchs Schenute von Atripe Tempel, ausgehend von der Thebais; vgl. Lib. or. 30,8-9. Vgl. PLRE I, 1971, s.v. Cynegius 3,235 f.; Frend 1990, 477, 482-483; De GiovanNI 1980, 143; HAHN 2004, 254 ff.

105 Von Theophilos' Vorgehen berichten Lib. or. 30,46-47; Rufin. h.e. 11, 28: per cunctas Aegypti urbes, per castella, per vicos, per omne rus, per ripas fluminis, per heremum quoque si qua fana vel potius busta repperiri potuerunt, instantia uniuscuiusque episcopi subruta et ad solum deducta sunt; Zos. 4,37,3; Consul. Constant. ad a. p. Chr. 388 (Chron. min. I,244: usque ad Aegyptum penetravit et simulacra gentium evertit); an die Zerstörung des religiösen Zentrums Kanopos erinnert Eun. vit. soph. 6,11. Ein sehr vielstimmiges Echo gab es auf die auf das Jahr 392 zu datierende Zerstörung des Sarapeions (das Sarapeion wurde für einen der wichtigsten Tempel gehalten, in seiner Bedeutung nur noch vom Jupiter-Tempel auf dem Kapitol in Rom übertroffen, wie bezeugt); vgl. Amm. 22,16,12; Hieron. epist. 107,2; Rufin. h.e. 11,23; 30; Eun. vit. 
Besonders detailliert im Codex Theodosianus aufgeführt ist das Gesetz vom 8. November 392 (Cod. Theod. 16,10,12), das das absolute Verbot zu opfern und den Kulten nachzugehen betonte und einige substantielle Strafen festlegte, wie die Konfiskation von omnia loca, falls das Delikt der violata religio begangen worden war. Ein Strafgeld war dagegen für den Fall vorgesehen, dass das Unrecht in templis fanisve publicis oder auf einem Privatgrundstück ohne Wissen des Eigentümers verübt worden war. Dieses Gesetz stellt einen wichtigen Bezugspunkt für die Rechtsstellung der Gebäude dar, die zum templum publicum und fanum publicum bestimmt worden waren. ${ }^{106}$ In der Stadt Rom stieß in der damaligen schwierigen politischen und kulturellen Situation die Anwendung einer solch drastischen Vorschrift auf Widerstand, insbesondere seitens führender städtischer Beamter, die weiterhin heidnischen Kulten anhingen, wie Rufius Albinus oder Virius Nicomachus Flavianus, die die kaiserlichen Vorschriften zur Restaurierung alter Gebäude anwandten, um die traditionellen Kultstätten intakt zu halten, und das sogar auf Kosten des Staates. ${ }^{107}$

Die Usurpation des Eugenius bedeutete eine kurze Pause in der antiheidnischen Politik, in der einige Vertreter des römischen Senats unter der Führung der Nicomachi heidnische Kulte wiederaufnehmen und Monumente der traditionellen Religion restaurieren konnten, darunter den Flora-Tempel in Rom und den Herkules-Tempel in Ostia. ${ }^{108}$ Zeichen des engen Bündnisses mit den heidnischen Senatoren ist im Jahr 394 die Ernennung von Virius Nicomachus Flavianus zum Konsul und seines Sohnes Virius Nicomachus Flavianus minor zum praefectus urbi. Der allgemeine gesetzliche Rahmen blieb jedoch antiheidnisch und Eugenius selbst vermied es, ihn zu ändern. Erwähnenswert in diesem Zusammenhang ist seine Vorsicht bei der Rückgabe konfiszierter Güter an die Tempel: Er stellte den Tempeln und den Kulten nicht direkt öffentliche Gelder zur Verfügung, sondern gewährte sie den heidnischen Senatoren in Form von Schenkungen, um nicht

soph. 6,11,1-5; Socr. h.e. 5,16,1; 7; Soz. h.e. 7,15,7-10; Thdt. h.e. 5,22,3. Vgl. FowdEN 1978, 70; BALDini 1985, 97 ff.; Frend 1990, 480; ChUvin 1990, 59 ff.; ThÉLAMON 1990, 525-526; 537; TeSTA 1991, 315-316; HAAS 1997, 207; HAHN 2004, 30-31; 80 ff.; 101-102. Für die Datierung siehe HAHN 2006 (mit Literatur insbesondere in Anm. 6).

106 Cod. Theod. 16,10,12: ...is utpote violatae religionis reus ea domo seu possessione multabitur, in qua eum gentilicia constiterit superstitione famulatum. Namque omnia loca, quae turis constiterit vapore fumasse, si tamen ea in iure fuisse turificantium probabuntur, fisco nostro adsocianda censemus. Sin vero in templis fanisve publicis aut in aedibus agrisve alienis tale quispiam sacrificandi genus exercere temptaverit...

107 Die Vorschrift, die vermutlich von Virius Nicomachus Flavianus verfasst wurde, als er quaestor sacri palatii war, wurde am 4. April 390 an Ceionius Rufius Albinus gerichtet (Cod. Theod. 15,1,27: Augustae in partibus civitatis magis antiqua reddi convenit quam inchoari supervaсиа); in diesem Zusammenhang vgl. LIZZI TESTA 2001, $700 \mathrm{ff}$.

108 Ihre Zahl wird im Curiosum Urbis Romae mit 300 angegeben (S. 40); vgl. FuHRMANN 1989, 101. 
gegen das mittlerweile gefestigte Verbot der öffentlichen Finanzierung der heidnischen Kulte zu verstoßen. ${ }^{109}$

Nach der Niederlage des Eugenius am Frigidus im Jahre 394 wurde die antiheidnische Gesetzgebung weiter verschärft; ${ }^{110}$ diesbezüglich sind zwei Gesetze erhalten, von denen das erste vom 7. August 395 die Orientierung der Religionspolitik von Theodosius I. bestätigte, ${ }^{111}$ während das zweite, das am 7. Dezember 396 an den praefectus praetorio Caesarius geschickt wurde, sämtliche Privilegien der heidnischen Priester abschaffte:

Privilegia si qua concessa sunt antiquo iure sacerdotibus ministris praefectis hierofantis sacrorum sive quolibet alio nomine nuncupantur, penitus aboleantur nec gratulentur se privilegio esse munitos, quorum professio per legem cognoscitur esse damnata. (Cod. Theod. 16,10,14).

Eine an den comes Orientis Asterius gerichtete Anordnung aus dem Jahre 397 behandelte ein schwerwiegendes und weit verbreitetes Problem in diesen Jahren, die Verwendung der Baumaterialien, die vom Abriss der Tempel herrührten (... cunctam materiam, quae ordinata dicitur ex demolitione templorum). Die Vorschrift bestätigte, dass sie öffentliches Gut seien, und legte fest, dass sie zur Instandhaltung und Ausbesserung von Straßen, Brücken, Aquädukten und Stadtmauern dienen sollten. ${ }^{112}$

In der Gesetzgebung des Jahres 399 bemerkt man den Übergang von einer Phase, in der die Gesetze einen gewissen Schutz der Tempelgebäude vorsahen, um das sich ausbreitende Phänomen der Zerstörungen, Plünderungen und Aneignungen zu beschränken, zu einer Phase, in der die Kaiser Zerstörungen und Konfiskationen ausdrücklich anordneten. Eine Verfügung des Honorius, die von Ravenna an die Stellvertreter des praefectus praetorio der Diözese Hispaniarum Macrobius und der Diözese Quinque provinciarum Proclianus geschickt wurde,

109 Diese Lösung wurde von Ambrosius scharf verurteilt; vgl. Ambr. ep. 57, 6; Paulin. v. Ambr. 26; PIGANIOL 1974, 293.

110 Nach dem Sieg wurden die von Eugenius am Ort der Auseinandersetzung aufgestellten ZeusStatuen zerstört; vgl. Aug. civ. 5,26. Über die Initiativen von Theodosius berichtet mit großer Genauigkeit Zos. 5,38,2 (nach dem Sieg über Eugenius kam Theodosius nach Rom, schaffte alle Priesterkollegien ab und verbot jede Form heidnischer Kulte); 3 (in dieser Atmosphäre erfolgte die frevelhafte Tat Serenas, die die Halskette von der Kultstatue der Magna Mater nehmen ließ, um sich selbst damit zu schmücken). Arcadius bemühte sich seinerseits, mit einem Gesetz vom August 395 (Cod. Theod. 16,10,13) zu betonen, dass die Anwendung der Vorschriften gegen Häretiker und Heiden verschärft werden würde: ... scituri quidquid divi genitoris nostri legibus est in ipsos vel supplicii vel dispendii constitutum, nunc acrius exequendum. Vgl. FLICHE MARTIN 1974, $551 \mathrm{ff}$;; 745 ff.; PASCHOUd 1975, 138 ff.; PASCHOUD 1986, $258 \mathrm{ff}$.

111 Cod. Theod. 16,10,13.

112 Cod. Theod. 15,1,36: man beachte den Begriff demolitio. Im Zusammenhang mit dieser Vorschrift steht Cod. Theod. 15,1,40 (vom 13. Dez. 398; vgl. Cod. Iust. 8,12,14), womit auch Privatpersonen zugestanden wurde, Baumaterialien zu verwenden, die für den öffentlichen Gebrauch nicht nutzbar waren: si aliquando operum publicorum petitores emergant, non nisi diruta penitusque destructa et quae parum sint usui civitatum petitoribus adsignentur; vgl. LIZZI TESTA 2001, 695, Anm. 69. 
enthielt letzte Sorgen um den Schutz der öffentlichen Gebäude; ${ }^{113}$ auch die Richtlinien vom 20. August desselben Jahres an den Prokonsul von Africa Apollodorus bestimmten, die traditionellen Feiertage nicht abzuschaffen und bestätigten das Verbot, die Tempel zu zerstören, wenn sie keine Kultgegenstände wie Statuen und Altare aufwiesen: ${ }^{114}$

Aedes inlicitis rebus vacuas nostrarum beneficio sanctionum ne quis conetur evertere. Decernimus enim, ut aedificiorum quidam sit integer status... (Cod. Theod. 16,10,18).

Eine anders ausgerichtete Politik verfolgte im Oströmischen Reich Arcadius, der mit einem am 10. Juli 399 in Damaskus erlassenen Gesetz anordnete, die Heiligtümer außerhalb der Stadt zu zerstören und lediglich verfügte, zur Vermeidung von Unruhen mit der notwendigen Vorsicht vorzugehen: ${ }^{115}$

Si qua in agris templa sunt, sine turba ac tumultu diruantur. His enim deiectis atque sublatis omnis superstitioni materia consumetur (Cod. Theod. 16,10,16).

Die Anwendung dieser Vorschrift hat sicherlich $\mathrm{zu}$ weiteren Turbulenzen und Spannungen geführt, in einer Zeit, die von Überfällen, Konfiskationen und Zerstörungen gekennzeichnet war. ${ }^{116}$ Der Codex Theodosianus enthält weitere Anordnungen sowohl in Bezug auf die Baumaterialien, die vom Abriss der Tempel herrührten, als auch in Bezug auf die Bestimmung der zu den Tempeln zugehörigen fundi. Mit einem am 26. November 400 in Mailand erlassenen Gesetz wird die Verpachtung verschiedener Arten öffentlichen Eigentums geregelt (Cod. Theod. 10,3,5: ea quae de iure templorum aut per diversos petita aut aeternabili domui fuerint congregata, vel civitatum territoriis ambiuntur...), darunter dieje-

113 Cod. Theod. 16,10,15 (29. Januar 399): sicut sacrifica prohibemus, ita volumus publicorum operum ornamenta servari ...; vgl. Hieron. ep. 107,2; Prud. c. Symm. 1,502. Zum Versuch eines versöhnenden Ausgleichs von Flavius Stilicho vgl. CASTELlo 1983, 65 ff.; DE GiovanNi 1980, $144 \mathrm{ff}$.

114 Cod. Theod. 16,10,17: Ut profanos ritus iam salubri lege submovimus, ita festos conventus civium et communem omnium laetitiam non patimur submoveri.

115 KUNDEREWICZ 1971, 146-147.

116 Zur Zerstörungswut der comites Flavius Iovinus und Gaudentius vgl. Anm. 118. In Phönizien zerstörten Mönchsbanden diverse Tempel, auch im Zuge der Agitation des Bischofs von Konstantinopel Johannes Chrysostomos; vgl. Jo. Chrys. ep. 126; 221; Thdt. h.e. 5,29. In Antiochia wurde der Tyche-Tempel in die Ignatioskirche verwandelt; vgl. Euagr. h.e. 1,16; Niceph. Call. h.e. 14,44; DeichmanN 1939, Anm. 2; Hahn 2004, 133. In Gaza ließ der Bischof Porphyrios die Tempel der Stadt zerstören, darunter das Marneion (24. Mai 403), eines der namhaftesten heidnischen Heiligtümer des Oströmischen Reichs; an dessen Stelle wurde die Hauptkirche der Stadt errichtet, die von der Kaiserin Eudoxia finanziert wurde. Die Erlaubnis zum Abriss des Tempels wurde vom Bischof Porphyrios über eine im Jahr 398 an den Hof von Konstantinopel entsandte und von Johannes Chrysostomos unterstützte Delegation gefordert; die Rolle der Kaiserin Eudoxia war dabei von großer Bedeutung; vgl. Marc. Diac. v. Porph. 26 ff.; 48 ff.; Soz. h.e. 7,15,14-15; DEICHMANN 1939, n. 15; 108-109; DEICHMANN 1954, 1230; KLEIN 1995, 134; RUBIN 1998, 50 ff.; HAHN 2004, 209 ff. (mit einer genauen Rekonstruktion der Bedingungen und Verantwortlichkeiten bei den Zerstörungen und Plünderungen sowie einer Darstellung der kulturellen und gesellschaftlichen Auswirkungen der Vorgehensweise des Bischofs). 
nigen de iure templorum, die ausschließlich für municipes, collegiatos et corporatos urbium singulorum bestimmt waren. ${ }^{117}$

Die Situation in der Provinz Africa, die durch das Vorgehen der circumcelliones, aber auch durch die zunehmenden Zerstörungen heidnischer Tempel, darunter des Tempels der Göttin Caelestis in Karthago, erschüttert wurde, erforderte eine Reihe kaiserlicher Verfügungen zum Schutz der öffentlichen Ordnung und der Reste der Gebäude der traditionellen Kulte. Die umfangreichen Vorschriften deuten daraufhin, dass die Verstrickung der Interessen an den heidnischen Heiligtümern noch recht bedeutend war. ${ }^{118}$ Im Konzil von Karthago des Jahres 401 wurde die systematische Zerstörung der Tempel und der Götzenbilder gefordert. Die Kaiser erließen daraufhin ein Rahmengesetz (Cod. Theod. 16,10,19), das am 15. November $407^{119}$ an den praefectus praetorio Curtius gerichtet wurde und mit dem

- den Tempeln die letzten Zuschüsse zur Finanzierung der Ausgaben für die Kulte und die epula sacra entzogen und den Soldaten zugewiesen wurden (templorum detrahantur annonae et rem annonariam iuvent expensis devotissimorum militum profuturae);

- die öffentlichen Tempelgebäude städtischen Nutzungszwecken zugewiesen wurden: aedificia ipsa templorum, quae in civitatibus vel oppidis vel extra oppida sunt, ad usum publicum vindicentur; ${ }^{120}$

- bestimmt wurde, die Tempel, die zum kaiserlichen Besitz gehörten, auf andere Art und Weise zu nutzen, während private Tempel zerstört werden mussten: omniaque templa in possessionibus nostris ad usus adcommodos transferantur; domini destruere cogantur;

- angeordnet wurde, die Altäre zu zerstören: arae locis omnibus destruantur;

- die Pflicht betont wurde, alle Statuen, die Kultgegenstände waren, zu entfernen: simulacra, si qua etiamnunc in templis fanisque consistunt et quae alicubi ritum vel acceperunt vel accipiunt paganorum, suis sedibus revellantur, cum hoc repetita sciamus saepius sanctione decretum.

Die Tatsache, dass die Bischöfe ausdrücklich angewiesen wurden, die Statthalter anzuzeigen, die es unterließen, die Einstellung der Ausübung jeglicher Kulte zu

117 DelmaiRe 1989, 657; GAUDEMET 1990, 462.

118 Die Zerstörung des Tempels der Göttin Caelestis erfolgte 399. Die comites Flavius Iovinus und Gaudentius zerstörten systematisch die Tempel in der Provinz Africa; vgl. Aug. serm 24,6; 52,12,18; Aug. ep. 93,8,26; 232,2; Aug. civ. 18,54. Vgl. LePELLEY 1979, 350 ff.; KLEIN 1995, 149 Anm. 79.

119 Vgl. Canon. 2,4 und 18; Cod. Afr. 58; 60; 84. Das Gesetz ist auf den 15. November 407 datiert; vgl. Const. Sirmond. 12; Demougeot 1950, 403-412; De Giovanni 1980, 146.

120 Indem die rechtliche Natur der Tempel als öffentliches Gut betont wurde, wurden die entsprechenden Schutzbestimmungen bestätigt; diese Vorschrift setzte nicht das Gesetz Cod. Theod. 16,10,15 aus dem Jahre 399 außer Kraft (Sicut sacrificia prohibemus, ita volumus publicorum operum ornamenta servari). Auch später wurden immer wieder Vorschriften zum Schutz der Baudenkmäler erlassen, wie zum Beispiel Nov. Maior. 4, 1 aus dem Jahre 458. 
überprüfen, beweist, mit welcher Entschlossenheit man die heidnischen Kulte in jeder Region des Reichs abschaffen wollte. ${ }^{121}$ Diese constitutio enthält jedoch auch eine Reihe genauer Hinweise zur Rechtsstellung und Bestimmung verschiedener Güter, die zu den Tempeln gehörten. Zuerst wird festgestellt, dass sowohl die Tempel in den oppida, als auch extra oppidis, öffentliches Eigentum und daher nach Beendigung der Nutzung als Kultstätte ad usum publicum vorzusehen seien; der Kaiser behielt sich dagegen das Recht vor, die Nutzung der Gebäude, die zum kaiserlichen Besitz gehörten, selbst zu bestimmen, während die Tempel, die sich auf privaten Grundstücken befanden, zerstört werden mussten.

Nicht weniger bedeutsam ist in rechtlicher Hinsicht das Schicksal der Kultgegenstände. Die Altäre mussten zerstört und die Kultstatuen entfernt werden, ohne dass ihre weitere Bestimmung angegeben wurde. Üblicherweise wurden sie zerstört (revellantur) oder außerhalb der Heiligtümer zur Stadtverschönerung verwendet.

In denselben Jahren (407-8) traf Stilicho einige antiheidnische Maßnahmen in Rom, um das Vertrauen unnachgiebiger Christen, die gegen seine Politik der Versöhnung Misstrauen hegten, zurückzugewinnen. Dazu zählten die Zerstörung der Sibyllinischen Bücher und die Entfernung der goldenen Beschläge der Türen des Kapitolinischen Tempels. ${ }^{122}$

Mit der constitutio Cod. Theod. 16,10,20, die am 30. August 415 von Honorius und Theodosius II. erlassen wurde, wurden sämtliche Immobilien, die von den heidnischen Kulten genutzt worden waren, von der res privata eingezogen, wobei man sich auf einen früheren Erlass Gratians berief (Omnia etiam loca, quae sacris error veterum deputavit, secundum divi Gratiani constituta, nostrae rei iubemus sociari) und betonte, dass dieser nicht nur für Africa gelte, sondern für das ganze Reich (Quod non tam per Africam quam per omnes regiones in nostro orbe positas custodiri decernimus): ${ }^{123}$

- alle fundi, deren Einkommen zur Finanzierung der Ausgaben für die Kulte bestimmt gewesen waren, wurden der res privata zugeleitet, außerdem wurden die unrechtmäßigen Erträge der vorhergehenden Jahre eingetrieben; ${ }^{124}$

- sämtliche Zuwendungen zu Gunsten der heidnischen Kulte (illius temporis superstitio, quae iure damnata est) wurden aufgehoben, wodurch die res privata entlastet wurde;

121 Cod. Theod. 16,10,19:... Episcopis quoque locorum haec ipsa prohibendi ecclesiasticae manus tribuimus facultatem; iudices autem viginti librarum auri poena constringimus ... si haec eorum fuerint dissimulatione neglecta.

122 Rut. Nam. 2,51-52; Oros. 7,38,6 (zur heidenfreundlichen Einstellung des Flavius Stilicho); Zos. 5,38,5; vgl. DEMANDT - BRunNER 1977, 496-497; PASCHOUd 1986, 266 ff. Siehe Anm. 110.

123 Vgl. Cod. Iust. 11,70,4; DE GIOVANNI 1980, 149-150.

124 Davon unberührt blieben die Schenkungen von den Kaisern an Privatpersonen. Die Rückerstattung der unrechtmäßigen Einkommen wurde in Bezug auf den Zeitpunkt berechnet, zu dem der Rechtsanspruch verloren gegangen war. Vgl. KLEIN 1995, 150, Anm. 83. 
- Einkommen von Immobilien (omnia loca), die heidnischen Vereinigungen zugeflossen waren, wurden von der res privata eingetrieben;

- in den Fällen, in denen das eingeschränkte Recht oder Verbot, Testamente zu Gunsten der Tempel zu verfassen, umgangen wurde, wurden die Güter durch fiscum nostrum für incrementa sacrarii nostri, fisci viribus beansprucht, unter der Zuständigkeit des officium rerum privatarum. ${ }^{125}$

Dass es sich um eine radikale Maßnahme handelte, wird sowohl durch die Vorschriften deutlich, die auch den letzten Formen des Herrscherkults ein Ende bereitete, indem die sacerdotales, die für den Herrscherkult in Africa verantwortlich waren, ihrer Ämter enthoben und aufgefordert wurden, zu einem bestimmten Datum den Sitz des concilium provinciale zu verlassen, ${ }^{126}$ als auch durch die Anordnung, sämtliche Gegenstände zu entfernen, die Ausdruck der traditionellen Kulte waren (Sane si quondam consecrata sacrificiis deceptionem hominum praestiterunt). In dieser Situation nahmen widerrechtliche Übergriffe und Amtsmissbrauch zu. Insbesondere sei an dieser Stelle die brutale Ermordung der Hypatia im Jahre 415 in Alexandria unter dem Episkopat des Kyrillos erwähnt. ${ }^{127}$ Aufgrund der Schwere des Amtsmissbrauchs musste der Gesetzgeber mit dem Edikt Cod. Theod. 16,10,24, das im Juni 423 an den praefectus praetorio Asklepiodotos gerichtet wurde, eingreifen. ${ }^{128}$ Das Edikt gibt über die einzelnen Tatbestände klare Auskunft:

- gewalttätige Handlungen waren sowohl von Christen als auch von Gruppen, die sich unrechtmäßigerweise als solche bezeichneten, begangen worden;

- es handelte sich um eine regelrechte Verfolgung auch von Heiden und Juden, welche die Gesetze einhielten;

- die Strafe für Personen, die sich schuldig gemacht hatten, bestand schlimmstenfalls in einer Entschädigung in beträchtlicher Höhe.

Mit dem Gesetz, das am 14. November 435 von den Kaisern Theodosius II. und Valentinian III. an den praefectus praetorio Flavius Anthemius Isidorus gerichtet wurde, wurde die letzte Anordnung gegen die heidnischen Tempel getroffen. Auch wenn der Hauptgrund die vollständige Entwurzelung des Heidentums war, ${ }^{129}$ wurde hiermit angeordnet, dass mit Hilfe der Magistrate sämtliche Gebäu-

125 Vgl. De Giovanni 1980, 158-159 (mit Bezug auf Cod. Theod. 16,5,49; 50; 58).

126 Am 1. November 408; vgl. KLEIN 1995, 150, Anm. 83.

127 Socr. h.e. 7,14-15; vgl. RougÉ 1990, 494 ff.; DZIELSKA 1995, 17 ff.; HAHN 2004, 110 ff.

128 Cod. Theod. 16,10,24 (8. Juni 423): ... Sed hoc Christianis, qui vel vere sunt vel esse dicuntur, specialiter demandamus, ut Iudaeis ac paganis in quiete degentibus nihilque temptantibus turbulentum legibusque contrarium non audean manus inferre religionis auctoritate abusi. Nam si contra securos fuerint violenti vel eorum bona diripuerint, non ea sola quae abstulerint, sed conventi in triplum et quadruplum quae rapuerint restituire conpellantur.

129 Die Gesetze des Jahres 423 vermitteln den Eindruck, dass das Heidentum nunmehr eine Randerscheinung sei: Cod. Theod. 16,10,22 (Paganos qui supersunt, quamquam iam nullos esse credamus...); Cod. Theod. 16,10,23 (Paganos qui supersunt). Letzteres Gesetz wandelt die für die Heiden vorgesehene Todesstrafe in Verbannung mit Enteignung um: Paganos qui supersunt, si 
de, die den heidnischen Kulten dienten, zu zerstören seien und an deren Stelle zur Buße die Symbole der christlichen Religion anzubringen seien: ${ }^{130}$

Cunctaque eorum fana templa delubra, si qua etiam nunc restant integra, praecepto magistratuum destrui conlocationeque venerandae Christianae religionis signi expiari praecipimus. (Cod. Theod. 16,10,25).

Die Prophezeiung der Tricennalienrede konnte sich nicht besser erfüllen: Die restlichen heidnischen Kultstätten wurden zerstört und durch die Symbole der siegreichen Religion ersetzt. ${ }^{131}$ Doch selbst diese pathetische Verfügung des Jahres 435 war nicht in der Lage, heidnische Traditionen völlig auszulöschen (noch der Hl. Benedikt vertrieb den Apollo-Kult vom Monte Cassino, der Parthenon wurde erst unter Justinian in eine Kirche verwandelt, ${ }^{132}$ und erst 609 erhielt Papst Bonifaz IV. von Phokas die Erlaubnis, das Pantheon der Madonna zu weihen ${ }^{133}$ ). Dennoch bildete sie den Abschluss der Gesetzgebung über die Tempelgüter, die zu diesem Zeitpunkt ihren Anspruch auf Rechtmäßigkeit verloren hatten und zu einem guten Teil entweder durch kaiserliche Verfügungen oder direkte Aneignung den Anhängern des siegreichen Gottes übertragen worden waren.

\section{Bibliographie}

Amerise, M., Eusebio di Cesarea. Elogio di Costantino. Discorso per il trentennale. Discorso regale, Mailand 2005.

Andreotti, R., Contributo alla discussione del rescritto Costantiniano di Hispellum, in: Problemi di storia e archeologia dell' Umbria Atti del I Convegno di Studi Umbri, Gubbio 1963, Perugia 1964, 249-290.

Arce, J., Reconstrucciones de templos paganos en época del emperador Juliano (361-363 d. C.), RSA 5 (1975), 201-215.

Arina, P., La legislazione di Giuliano, AAN 46 (1985), 197-239.

Armstrong, A.H., The Way and the Ways: Religious Tolerance and Intolerance in the Fourth Century A.D., VigChr 38 (1984), 1-17.

aliquando in execrandis daemonum sacrificiis fuerint deprehensi, quamvis capitali poena subdi debuerint, bonorum proscriptio ac exilium cohercebit. Vgl. FLICHE - MARTIN 1974, 4.3, 24.

130 Deichmann 1954, 1229 ff. Eine späte Initiative zum Schutz der noch erhaltenen Tempel, wie auch anderer öffentlicher Gebäude, war die Novella 4 von Maiorianus, die nach der Plünderung Roms durch Geiserich am 11. Juli 458 an den praefectus urbi geschickt wurde.

131 In jenen Jahren feierte Theodoret von Kyrrhos in einer seiner Apologien die vollkommene Bedeutungslosigkeit der heidnischen Tempel und die „Widmung“ der Materialien zum Bau der Märtyrer-Gedenkstätten; auch bezüglich der Anteilnahme des Volkes an den neuen Ritualen der Feiertage verherrlichte er den Ersatz der traditionellen durch die christlichen Feste; vgl. Thdt. affect. 8,49 (PG 83, 1033); TESTA 1991, 319-320.

132 Der terminus ante quem für die Umwandlung des Parthenons in eine Basilika ist die Regentschaft Justinians; auf das Jahr 430 geht jedoch ein Traum des Neuplatonikers Proklos zurïck, der im Äskulap-Heiligtum wohnte, wo ihm die Göttin Athene erschien und um Aufnahme in sein Haus bat; vgl. Marin. v. Procli 29-30; FRANTZ 1965, 194; PAVAN 1983, 33.

133 Lib pontif. 68,2 (Duchesne). 
Athanassiadi Fowden, P., Julian and Hellenism: An Intellectual Biography, Oxford 1981.

Baldini, A., Problemi della tradizione sulla 'distruzione' del Serapeo di Alessandria, RSA 15 (1985), 97-152.

Barb, A.A., The Survival of Magic Arts, in: Momigliano, A. (Hrsg.), The Conflict between Paganism and Christianity in the Fourth Century, London 1963, 100-125.

Barnard, L.W., L'intolleranza negli apologisti cristiani con speciale riguardo a Firmico Materno, CrSt 11 (1990), 505-521.

Barnes, T.D., Constantine and Eusebius, Cambridge, MA - London 1981.

Barnes, T.D., Constantine's Prohibition of Pagan Sacrifice, AJPh 105 (1984), 69-72.

Barnes, T.D., Panegyric, History and Hagiography in Eusebius' Life of Constantine, in: Williams, R. (Hrsg.), The Making of Orthodoxy: Essays in Honour of Henry Chadwick, Cambridge 1989a, 94-123.

Barnes, T.D., Christians and Pagans in the Reign of Constantius, in: Vittinghoff, F. Dihle, A. (Hrsg.), L'Église et l'empire au IV ${ }^{\mathrm{e}}$ siècle (Entretiens Hardt 34), Vandoeuvres - Genf 1989b, 301-343.

Barsanti, C., Costantinopoli: testimonianze archeologiche di età costantiniana, in: Bonamente, G. - Fusco, F. (Hrsg.), Costantino il Grande. Dall'antichità all' Umanesimo. Colloquio sul cristianesimo nel mondo antico, Bd. I, Macerata 1992, 115-150.

Beatrice, P.F. (Hrsg.), L'intolleranza cristiana nei confronti dei pagani, CrSt 11 (1990), 441-615.

Blanchetière, F., La législation antijuive de Théodose II, Ktèma 6 (1981), 125-129.

Bloch, H., The Pagan Revival in the West at the End of the Fourth Century, in: Momigliano, A. (Hrsg.), The Conflict between Paganism and Christianity in the Fourth Century, Oxford 1963, 193-218.

Bodei Giglioni, G., Pecunia fanatica. L'incidenza economica dei templi laziali, RSI 89 (1977), 33-76.

Bonamente, G., Considerazioni sul 'De rebus bellicis', AFLM 14 (1981), 9-49.

Bonamente, G., Le città nella politica di Giuliano l'Apostata, AFLM 16 (1983), 37-101.

Bonamente, G., Giuliano l'Apostata e il 'Breviario' di Eutropio, Rom 1986.

Bonamente, G., Sulla confisca dei beni mobili dei templi in epoca costantiniana, in: Bonamente, G. - Fusco, F. (Hrsg.), Costantino il Grande. Dall'antichità all'Umanesimo. Colloquio sul cristianesimo nel mondo antico, Bd. I, Macerata 1992, 171- 201.

Bonamente, G., Chiesa e impero nel IV secolo: Costanzo II fra il 357 e il 361, in: Pani Ermini, L. - Siniscalco, P. (Hrsg.), Comunità cristiana di Roma. La sua vita e la sua cultura dalle origini all' Alto Medio Evo, Rom 2000, 113-138.

Bonamente, G., Prefetti del pretorio, vescovi e governatori all'opera nell'applicare la legislazione antipagna, in: Di Paola, L. - Minutoli, D. (Hrsg.), Poteri centrali e poteri periferici nella tarda antichità. Confronti conflitti. Atti della Giornata di Studio Messina, 5 Settembre 2006, Papyrologica Florentina 38 (2007), 13-34

Bowersock, G.W., Julian the Apostate, Cambridge 1980.

Bradbury, S., Constantine and the Problem of Anti-Pagan Legislation in the Fourth Century, CPh 89 (1994), 120-139.

Brandt, H., Zeitkritik in der Spätantike. Untersuchungen zu den Reformvorschlägen des Anonymus De rebus bellicis, München 1988.

Brandt, H., La politica fiscale di Costantino nelle opinioni tardoantiche, in: Bonamente, G. - Fusco, F. (Hrsg.), Costantino il Grande. Dall'antichità all'Umanesimo. Colloquio sul cristianesimo nel mondo antico, Bd. I, Macerata 1992, 213-218. 
Brennecke, H.Ch., Hilarius von Poitiers und die Bischofsopposition gegen Konstantius II. Untersuchungen zur dritten Phase des arianischen Streites (337-361), Berlin - New York 1984.

Bringmann, K., Die Konstantinische Wende. Zum Verhältnis von politischer und religiöser Motivation, HZ 260 (1995), 21-47.

Calderone, S., Da Costantino a Teodosio, in: Nuove questioni di Storia Antica, Mailand 1968, 615-684.

Callu, J.-P., Problèmes monetaires du quatrième siècle (311-395), in: Transformations et conflicts au IV siècle ap. J.-C., Colloque, Bordeaux 7-12 sept. 1970, Bonn 1978, 103126.

Callu, J.-P. - Marandon, J.-N., L'inflazione nel IV secolo (295-361): il contributo delle analisi, in: Giardina A. (Hrsg.), Società romana e impero tardoantico, Bd. I, Rom Bari 1986, 559-599.

Callu, J.-P., Succès et limites du solidus constantinien, in: Urso, G. (Hrsg.), Moneta, mercanti, banchieri. I precedenti greci e romani dell'Euro. Atti del convegno internazionale Cividale del Friuli 2002, Pisa 2003, 205-218.

Caltabiano, M., L'assassinio di Giorgio di Cappadocia (Alessandria, 361 d. C.), CQ 7 (1985), 17-59.

Caltabiano, M., L'epistolario di Giuliano imperatore (AST 14), Napoli 1991.

Cameron, A. - Hall, S.G., Eusebius, Life of Constantine: Introduction, Translation and Commentary, Oxford 1999.

Castello, C., L'umanesimo cristiano di Stilicone, in: Atti dell Accademia Romanistica Costantiniana in onore di M. de Dominicis, Perugia 1983, 65-96.

Cataudella, M.R., Aurum pro aere nella politica di Costantino, in: Bonamente, G. - Fusco, F. (Hrsg.), Costantino il Grande. Dall' antichità all'Umanesimo. Colloquio sul cristianesimo nel mondo antico, Bd. I, Macerata 1992, 283-297.

Chastagnol, A., La préfecture urbaine à Rome sous le Bas-Empire, Paris 1960.

Chastagnol, A., Les Fastes de la Préfecture de Rome au Bas-Empire, Paris 1962.

Chastagnol, A., La restauration du temple d'Isis au 'Portus Romae' sous le regne de Gratien, in: Hommages à M. Renard, Bd. II, Brüssel 1969, 135-144.

Chuvin, P., Chronique des dernier païens: la disparition du paganisme dans l'empire romain, du règne de Constantin à celui de Justinien, Paris 1990.

Coarelli, F., Il rescritto di Spello e il santuario 'etnico' degli Umbri, in: Umbria cristiana. Dalla diffusione del culto al culto dei santi (secc. IV-X). Atti del XV Congresso internazionale di studi sull'alto medioevo, Spoleto 2000, Bd. I, Spoleto 2001, 39-56.

Condurachi, E., La politique financière de l'empereur Julien, Bukarest 1941.

Cracco Ruggini, L., Esperienze economiche e sociali nel mondo romano, in: Nuove questioni di storia antica, Mailand 1969, 685-813.

Cracco Ruggini, L., Il paganesimo romano fra religione e politica (384-394 d. C.): per una reinterpretazione del 'Carmen contra paganos', MANL (Cl. Sc. Mor., St. e Filol.) VIII 23,1 (1979), 1-144.

Cracco Ruggini, L., Pagani, Ebrei, Cristiani: odio sociologico e odio teologico nel mondo antico, in: XXVI Settimana di Studio sull'Alto Medioevo, 1978, Bd. I, Spoleto 1980, $15-101$.

Cracco Ruggini, L., La città nel mondo antico: realtà e idea, in: Wirth, G. - Schwarte, K.H. - Heinrichs, J. (Hrsg.), Romanitas - Christianitas. Untersuchungen zur Geschichte und Literatur der römischen Kaiserzeit, Berlin - New York 1982, 78 ff. 
[Cracco] Ruggini, L., Economia e società nell'Italia Annonaria. Rapporti fra agricoltura e commecio dal IV al VI secolo d. C., Bari ${ }^{2} 1995$.

Cuneo, P.O., La legislazione di Costantino II, Costanzo II e Costante (337-361), Mailand 1997.

Dagron, G., Naissance d'une capitale. Constantinople et ses institutions de 330 à 451, Paris 1974 [ $\left.^{2} 1984\right]$.

Dagron, G., Constantinople imaginaire. Études sur le recueil des Patria, Paris 1984.

De Giovanni, L., Chiesa e Stato nel codice Teodosiano. Saggio sul libro XVI, Neapel 1980.

De Giovanni, L., Diritto romano tardoantico. Lezioni, Neapel ${ }^{5} 2001$.

De Giovanni, L., L'imperatore Costantino e il mondo pagano, Neapel ${ }^{2} 2003$.

Deichmann, F.W., Frühchristliche Kirchen in antiken Heiligtümern, JDAI 54 (1939), 105136.

Deichmann, F.W., Christianisierung I, in: RAC 2 (1954), 1228-1241.

Deichmann, F.W., Die Spolien in der spätantiken Architektur, München 1975.

De Labriolle, P., La reaction païenne, Paris 1934.

Demandt, A. - Brummer, G., Der Prozess gegen Serena im Jahre 408 n. Chr., Historia 26 (1977), 479-502.

Demougeot, E., Sur les lois du 15 novembre 407, RHD 1950, 403-412.

Downey, G., A History of Antioch in Syria from Seleucus to the Arab Conquest, Princeton 1961.

Drake, H.A., When Was the De laudibus Constanti Delivered? Historia 24 (1975), 345356.

Drake, H.A., In Praise of Constantine. A Historical Study and New Translation of Eusebius' Tricennial Orations, Berkeley - Los Angeles 1976.

Dummer, J., Fl. Artemius dux Aegypti, APF 21 (1971), 121-144.

Dzielska, M., Hypatia of Alexandria, Cambridge 1995.

Edbrooke, R.O., The Visit of Constantius II to Rome in 357 and Its Effect on the Pagan Roman Senatorial Aristocracy, AJPh 97 (1976), 40-61.

Ensslin, W., Die Religionspolitik des Kaisers Theodosius des Großen, München 1953.

Errington, R M., Constantine and the Pagans, GRBS 29 (1988), 309-318.

Errington, M.R., Christian Accounts of the Religious Legislation of Theodosius I, Klio 79 (1997), 398-443.

Festugière, A.J., Antioche païenne et chrétienne. Libanius, Chrysostome et les moines de Syrie, Paris 1959.

Fliche, A. - Martin, V., Storia della Chiesa, Bd. III,2, Turin 1974.

Forni, G., Flavia Constans Hispellum. Il tempio e il pontefice della gens Flavia costantiniana (1992), in: Scritti vari di storia, epigrafia e antichità romane, Bd. I, Rom 1994, 257-261.

Foschia, L., La réutilisation des sanctuaires païens par les chrétiens en Gréce continentale (IV ${ }^{\mathrm{e}}-\mathrm{VII}^{\mathrm{e}}$ s.), REG 113 (2000), 413-434.

Fowden, G., Bishops and Temples in the Eastern Roman Empire A.D. 320-435, JThS 29 (1978), 53-78.

Frantz, A., From Paganism to Christianity in the Tempels of Athens, DOP 19 (1965), 185205.

Fraschetti, A., La conversione. Da Roma pagana a Roma cristiana, Bari 1999.

Frend, W.H.C., Monks and the End of Greco-Roman Paganism in Syria and Egypt, CrSt 11 (1990), 469-484. 
Fuhrmann, M., Das Verzeichnis der Regionen von Rom, in: Herzog, P. (Hrsg.), Restauration und Erneuerung. Die lateinische Literatur 284-374 n. Chr. (Handbuch der lateinischen Literatur des Altertums 5), München 1989, 99-100.

Gascou, J., Le rescrit d'Hispellum, MEFR 79 (1967), 609-659.

Gaudemet, J., L'Église dans l'empire romain (IV et V $\mathrm{V}^{\mathrm{e}}$ siècles), Paris 1958.

Gaudemet, J., Politique écclésiastique et législation religieuse après l'Édit de Théodose $\mathrm{I}^{\mathrm{er}}$ de 380, Atti Accad. Rom. Costant. 6 (1986), 1-22.

Gaudemet, J., La législation anti-païenne de Constantin à Justinien, CrSt 11 (1990), 449468.

Gaudemet, J., La politique religieuse impériale au $\mathrm{IV}^{\mathrm{e}}$ siècle (envers les païens, les Juifs, les hérétiques, les donatistes), in: Gaudemet, J. - Siniscalco, P. - Falchi, G.L. (Hrsg.), Legislazione imperiale e religione nel IV secolo, Rom 2000, 7-66.

Geffcken, J.G., Der Ausgang des griechisch-römischen Heidentum, Heidelberg 21929.

Giardina, A. (Hrsg.), Società romana e impero tardoantico, I-IV, Rom - Bari 1986.

Giardina, A. (Hrsg.), Anonimo. Le cose della guerra, Mailand ${ }^{3} 1996$.

Girardet, K.M., Die Petition der Donatisten an Kaiser Konstantin (Frühjahr 313). Historische Voraussetzungen und Folgen, Chiron 19 (1989), 185-206.

Girardet, K.M., Die Konstantinische Wende und ihre Bedeutung für das Reich. Althistorische Überlegungen zu den geistigen Grundlagen der Religionspolitik Konstantins des Großen, in: Mühlenberg, E. (Hrsg.), Die Konstantinische Wende, Gütersloh 1998, 9122.

Guyon, J., Roma. Emerge la città cristiana, in: Carandini, A. - Cracco Ruggini, L. - Giardina, A. (Hrsg.), Storia di Roma, Bd. III,2, Turin 1993, 53-68.

Haas, C., The Alexandrian Riots of 356 and George of Cappadocia, GRBS 32 (1991), 281-301.

Haas, C., Alexandria in Late Antiquity: Topography and Social Conflict, Baltimore 1997.

Hahn, J., Tempelzerstörung und Tempelreinigung, in: Albertz, R. (Hrsg.), Kult, Konflikt, Sühne (Veröffentlichungen des Arbeitskreises zur Erforschung der Religions- und Kulturgeschichte des Antiken Vorderen Orients 2), Münster 2000, 269-285.

Hahn, J., Gewalt und religiöser Konflikt. Studien zu den Auseinandersetzungen zwischen Christen, Heiden und Juden im Osten des römischen Reiches (von Konstantin bis Theodosius II.) (Klio-Beihefte n. F. 8), Berlin 2004.

Hahn, J., Vetus error extinctus est - Wann wurde das Sarapeion von Alexandria zerstört? Historia 55 (2006), 368-383.

Hahn, J., The Conversion of the Cult Statues: The Destruction of the Serapeion 392 A.D. and the Transformation of Alexandria into the „Christ-Loving City“, in: Hahn, J. Emmel, St. - Gotter, U. (Hrsg.), From Temple to Church: Destruction and Renewal of Local Cultic Topography in Late Antiquity (Religions in the Graeco-Roman World 163), Leiden 2008 (im Druck).

Hanson, R.P.C., The Transformation of Pagan Temples into Churches in the Early Christian Centuries, Journal of Semitic Studies 23 (1978), 257-267.

Hanson, R.P.C., The Christian Attitude to Pagan Religions up to the Time of Constantine the Great, ANRW II 23,2 (1980), 910-973.

Irmscher, J., La politica religiosa dell' imperatore Giustiniano, CrSt 11 (1990), 579-592.

Jones, A.H.M., The Development of Constantine's Attitude towards Paganism, in: Atti X Congr. intern. Scienze Storiche, Roma 4-11 sett., 1955, Bd. VIII, Rom 1957, 267271. 
Jones, A.H.M., The Later Roman Empire (284-602). A Social, Economic and Administrative Survey, Oxford 1972.

Klein, R., Constantius II. und die christliche Kirche, Darmstadt 1977.

Klein, R., Der Rombesuch des Kaisers Konstantius II. im Jahre 357, Athenaeum 57 (1979), 98-115.

Klein, R., Distruzione di templi nella tarda antichità. Un problema politico, culturale e sociale, in: Atti del X Convegno intern. Accademia Romanistica Costantiniana, 1991, Perugia 1995, 127-152.

Kolb, F., Finanzprobleme und soziale Konflikte aus der Sicht zweier spätantiker Autoren (Scriptores Historiae Augustae und Anonymus de rebus bellicis), in: Eck, W. (Hrsg.), Studien zur antiken Sozialgeschichte. Festschrift F. Vittinghoff, Köln - Wien 1980, 497-525.

Krautheimer, R., The Ecclesiastical Building Policy of Constantine, in: Bonamente, G. Fusco, F. (Hrsg.), Costantino il Grande. Dall'antichità all’Umanesimo. Colloquio sul cristianesimo nel mondo antico, Bd. II, Macerata 1993, 509-552.

Kunderewicz, C., La protection des monuments d'architecture antique dans le Code Théodosien, in: Studi in onore di E. Volterra, Bd. IV, Milano 1971, 137-153.

Lane Fox, R., Pagans and Christians, London - New York 1984.

La Rocca, E., La fondazione di Costantinopoli, in: Bonamente, G. - Fusco, F. (Hrsg.), Costantino il Grande. Dall' antichità all'Umanesimo. Colloquio sul cristianesimo nel mondo antico, Bd. II, Macerata 1993, 553-583.

Latte, K., Römische Religionsgeschichte, München 1960.

Lepelley, C., Les cités de l'Afrique romaine au Bas-Empire, Bd. I, Paris 1979.

Leppin, H., Constantius II. und das Heidentum, Athenaeum 87 (1999), 457-480.

Liebeschuetz, J.H.W.G., Antioch. City and Imperial Administration in the Later Roman Empire, Oxford 1972.

Lizzi, R., Vergini di Dio - vergini di Vesta. Il sesso negato e la sacralità, in: Pricoco, S. (Hrsg.), L'Eros difficile. Amore e sessualità nell'antico cristianesimo, Soveria Mannelli 1998, 89-132.

Lizzi Testa, R., The Bishop, 'Vir Venerabilis': Fiscal Privileges and 'Status' Definition in Late Antiquity, StudPatr 34 (2001a), 125-144.

Lizzi Testa, R., Paganesimo politico e politica edilizia: la 'cura Urbis' nella tarda antichità, in: Centralismo e autonomie nella tarda antichità. Categorie concettuali e realtà concrete (Perugia-Spello 1997), AARC 13 (2001b), 671-707.

Lizzi Testa, R., Senatori, popolo, papi. Il governo di Roma al tempo dei Valentiniani, Bari 2004.

Lizzi Testa, R., Martino vescovo santo: un nuovo modello di santità nell' Occidente tardoantico (im Druck).

Lo Cascio, E., Teoria e politica monetaria a Roma tra III e IV d. C., in: Giardina, A. (Hrsg.), Società romana e impero tardoantico, Bd. I, Rom - Bari 1986, 535-557.

MacMullen, R., Roman Imperial Building in the Provinces, HSPh 64 (1959), 207-235.

MacMullen, R., Christianizing the Roman Empire A.D. 100-400, New Haven - London 1984.

Mango, C., Ancient Statuary and Byzantine Beholder, DOP 17 (1963), 53-75.

Marasco, G., L'imperatore Giuliano e l'esecuzione di Fl. Artemio, dux Aegypti, Prometheus 23 (1997), 59-78.

Maraval, P., La théologie politique de l'empire chrétien. Louanges de Constantin, Paris 2001. 
Masi, A., Ricerche sulla 'res privata' del princeps, Mailand 1971.

Mazzarino, S., Aspetti sociali del IV secolo. Ricerche di storia tardo-romana, Rom 1951a.

Mazzarino, S., La propaganda senatoriale nel tardo impero, Doxa 5 (1951b), 121ff.

Mazzarino, S., Il Carmen 'Contro i pagani' e il problema dell' "Era Costantiniana", ATA I, Rom - Bari 1974a.

Mazzarino, S., Tolleranza e intolleranza. La polemica sull'ara della Vittoria, in Antico, tardoantico ed èra costantiniana, Bd. I, Bari 1974b, 339-377.

Meier, H.-R., Alte Tempel - neue Kulte. Zum Schutz obsoleter Sakralbauten in der Spätantike und zur Adaptation alter Bauten an den christlichen Kult, in: Brenk, R. (Hrsg.), Innovation in der Spätantike. Kolloquium Basel 1994, Wiesbaden 1996, 363-376.

Metzler, D., Ökonomische Aspekte des Religionswandels in der Spätantike: Die Enteignung der heidnischen Tempel seit Konstantin, Hephaistos 3 (1981), 27-40.

Mittag, F.P., Alte Köpfe in neuen Händen. Urheber und Funktion der Kontorniaten (Antiquitas, 3. Reihe, 38), Bonn 1999.

Moreschini, C., Quando un imperatore cristiano perseguita i cristiani, in: Dal Covolo, E. Uglione, R. (Hrsg.), Cristianesimo e istituzioni politiche, Bd. II: Da Costantino a Giustiniano (Biblioteca di scienze religiose 134), Rom 1997, 107-126.

Noethlichs, K.L., Die gesetzgeberischen Maßnahmen der christlichen Kaiser des 4. Jh. gegen Häretiker, Heiden und Juden, Diss. Köln 1971.

Noethlichs, K.L., Heidenverfolgung, in: RAC 14 (1986), 1149-1196.

Onida, P.P., Il divieto dei sacrifici di animali nella legislazione di Costantino. Una interpretazione sistematica, in: Sini, F. - Onida, P.P. (Hrsg.), Poteri religiosi e istituzioni: il culto di San Costantino imperatore tra Oriente e Occidente, Turin 2003, 73-174.

Palanque, J.R., Saint Ambroise et l'empire romain, Paris 1933.

Pani Ermini, L. - Siniscalco, P. (Hrsg.), La comunità cristiana di Roma. La sua vita e la sua cultura dalle origini all' alto Medioevo, Vatikanstadt 2000.

Paschoud, F., Cinq études sur Zosime, Paris 1975.

Paschoud, F., Un problème de circulation monétaire au IV ${ }^{\mathrm{e}}$ siècle après Jésus-Christ, in: Mélanges offerts à P. Collart, Lausanne 1976, 307-316.

Paschoud, F., Le rôle du providentialisme dans le conflit de 384 sur l'autel de la Victoire, MH 40 (1983), 197-206.

Paschoud, F. (Hrsg.), Actes du Colloque pour le $1600^{\mathrm{e}}$ anniversaire du débat autour de l'autel de la Victoire, Genève 1984, Paris 1986a.

Paschoud, F. (Hrsg.), Zosime. Histoire nouvelle. Livre V, Bd. III,1, Paris 1986b.

Paschoud, F., L'intolérance chrétienne vue et jugée par les païens, CrSt 11 (1990), 545577.

Pavan, M., I cristiani e il mondo ebraico nell'età di Teodosio il Grande, AFLPer 3 (19651966), 367-530.

Pavan, M., L'avventura del Partenone. Un monumento nella storia, Florenz 1983.

Petit, P., Sur la date du 'Pro templis' de Libanius, Byzantion 21 (1951), 285-309.

Petit, P., Libanius et la vie municipale à Antioche au IV siècle après Jésus-Christ, Paris 1955.

Pietri, Ch., Roma christiana. Recherches sur l'Église de Rome, son organisation, sa politique, son idéologie de Miltiade à Sixte III (311-447), Bde. I-II, Rom 1976.

Pietri, Ch., La politique de Constance II: un premier 'césaropapisme' ou l'imitatio Constantini? in: L'Église et l'empire au IV ${ }^{\mathrm{e}}$ siècle (Entretiens Hardt 34), Vandoeuvres Genf 1989, 113-178 [ND in: PIETRI 1997, Bd. I, Rom 1997, 281-346]. 
Pietri, Ch., Christiana respublica. Éléments d'une enquête sur le christianisme antique, Bde. I-III, Rom 1997.

Piganiol, A., L'empire chrétien (325-395), Paris 1974.

Romano, R., Libanio. In difesa dei templi, Neapel 1982.

Rosen, K., Ilario di Poitiers e la relazione tra la chiesa e lo stato, in: Bonamente, G. Nestori, A. (Hrsg.), I cristiani e l'impero nel IV secolo. Colloquio sul cristianesimo nel mondo antico, Macerata 1988, 63-74.

Rosen, K., Fides contra dissimulationem. Ambrosius und Symmachus im Kampf um den Victoriaaltar, JbAC 37 (1994), 29-36.

Rougé, J., La politique de Cyrille d'Alexandrie et le meurtre d'Hypatie, CrSt 11 (1990), 485-504.

Rubin, Z., Porphyrius of Gaza and the Conflict between Christianity and Paganism in Southern Palestine, in: Kofsky, A.- Stroumsa, G.G. (Hrsg.), Sharing the Sacred, Jerusalem 1998, 31-66.

Sabbah, G. (Hrsg.), Sozomène. Histoire ecclésiastique. Livres I-II (Sources chrétiennes 306), Paris 1983.

Salzman, M.R., 'Superstitio' in the Codex Theodosianus and the Persecution of Pagans, VChr 41 (1987), 172-188.

Salzman, M.R., On Roman Time. The Calendar of 354 and the Rhythms of Urban Life in Antiquity (The Transformation of the Classical Heritage 17), Berkeley - Los Angeles - Oxford 1990.

Schweitzer, C., Hierarchie und Organisation der römischen Reichskirche in der Kaisergesetzgebung vom 4. zum 6. Jh., Frankfurt a. M. 1991.

Sievers, G.R., Das Leben des Libanius, Berlin 1868 [ND Amsterdam 1969].

Simon, M., Verus Israel. Étude sur les relations entre chrétiens et juifs dans l'empire romain (135-425), Paris ${ }^{2} 1964$.

Stemberger, G., Juden und Christen im Heiligen Land. Palästina unter Konstantin und Theodosius, München 1987.

Stewart, P., The Destruction of Statues in Late Antiquity, in: Miles, R. (Hrsg.), Constructing Identities in Late Antiquity, London - New York 1999, 159-189.

Testa, E., Legislazione contro il paganesimo e cristianizzazione dei templi (sec. IV-VI), Liber Annus 41 (1991), 311-326.

Thélamon, F., Destruction du paganisme et construction du royaume de Dieu d'après Rufin et Augustin, CrSt 11 (1990), 523-544.

Trombley, F., Hellenic Religion and Christianization c. 370-529 (Religions in the GraecoRoman World 115), Bde. I-II, Leiden 1993-1994.

Tsafrir, Y., The Fate of Pagan Cult Places in Palestine, in: Lapin, H. (Hrsg.), Religious and Ethnic Communities in Later Roman Palestine, Bethesda, MD 1998, 197-218.

Turcan, R., Firmicus Maternus. De l'erreur des religions païennes, Paris 1982.

Vera, D., Commento storico alle Relazioni di Quinto Aurelio Simmaco, Pisa 1981.

Vogler, C., Les Juifs dans le code théodosien, Paris 1979.

von Lasaulx, E., Der Untergang des Hellenismus und die Einziehung seiner Tempelgüter durch die christlichen Kaiser. Ein Beitrag zur Philosophie der Geschichte, München 1856.

Warmington, B.H., Eusebius of Caesarea's Version of Constantine's Laws in the Codes, StudPatr 24 (1993), 201-207.

Weis, B.K., Das Restitutionsedikt Kaiser Julians, Heidelberg 1933. 
Wiemer, H.-U., Die Rangstellung des Sophisten Libanios unter den Kaisern Julian, Valens und Theodosius. Mit einem Anhang über Abfassung und Verbreitung von Libanios' Rede Für die Tempel (Or. 30), Chiron 25 (1995), 89-130.

Wrede, H., Die spätantike Hermengalerie von Welschbillig, Berlin 1972. 


\title{
Mala desidia iudicum? \\ Zur Rolle der Provinzstatthalter bei der Unterdrückung paganer Kulte (von Constantin bis Theodosius II.) ${ }^{*}$
}

\author{
ECKHARD MEYER-ZWIFFELHOFFER
}

\section{Einleitung: Religiöse Konflikte in der Provinz Africa}

Am 5. Juni des Jahres 408 ließ der Prokonsul der Provinz Africa, Porphyrius, auf dem Forum der Provinzhauptstadt Carthago ein Gesetz des Kaisers Honorius anschlagen, das dieser am 25. November des Vorjahres in Rom für den Prätorianerpräfekten von Italien und Africa, Curtius, ausgestellt hatte: ${ }^{1}$

Genötigt also durch die Hartnäckigkeit der Donatisten und den Wahnsinn der Heiden, welche freilich von der verderblichen Untätigkeit der Statthalter (mala desidia iudicum), der Kollaboration in den Statthalterbüros und der Missachtung der Kurialen angefacht wurden, sehen wir uns gezwungen zu wiederholen, was wir schon früher befohlen haben.

Mit diesen Worten leitet der Kaiser nach einer Proklamation seiner religionspolitischen Aufgabe zu den Maßnahmen gegen Häretiker und ,Heiden ${ }^{2}$ über, die er

* Erweiterte Fassung meines Vortrags auf dem Kolloquium. Für eine kritische Lektüre des Manuskripts und weiterführende Hinweise danke ich herzlich Johannes Hahn. Das Manuskript wurde im März 2006 abgeschlossen.

1 Const. Sirmond. 12: conpulsi igitur Donatistarum pertinacia, furore gentilium, quae quidem mala desidia iudicum, coniventia officiorum, ordinum contemptus accendit, necessarium putamus iterare quae iussimus - Exzerpte aus dieser Konstitution wurden in den Codex Theodosianus aufgenommen; die Maßnahmen gegen die Donatisten unter 16, 5 [de haereticis], 43, diejenigen gegen die ,Heiden“ unter 16, 10 [de paganis, sacrificiis et templis], 19. C. Aelius Pompeius Porphyrius Proculus ist als Prokonsul bezeugt zwischen dem 15. November 407 und dem 5. Juni 408 (PLRE II 900-901, Porphyrius 3), Curtius als Prätorianerpräfekt zwischen dem 7. April 407 und dem 3. Februar 408 (PLRE II 331). - Zum Gesetzesbegriff im Codex Theodosianus siehe unten Anm. 53.

2 Alle als sectae bezeichneten religiösen Gruppierungen einschließlich der catholica secta sind ein Resultat der Formierung der christlichen Orthodoxie: Dies ist evident für die so genannten häretischen Bewegungen, deren Etikett zumeist vom Namen einer prominenten Gründerfigur abgeleitet wurde (Eunomianer, Priscillianisten, Arianer, Donatisten). Dies ist gleichermaßen evident in der christlichen Definition aller ,Heiden“ (mit Ausnahme der Juden und Manichäer) als pagani bzw. gentiles, woraufhin dann Kaiser Julian versuchte, diese Identität positiv zu begründen. Dass dies auch für die ,Juden“ gilt, hat kürzlich BOYARIN 2004 dargelegt. Der definito- 
erneut ein- und zugleich verschärft. Alle bislang von den Kaisern der theodosianischen Dynastie erlassenen generales leges sollen nicht nur in Kraft bleiben, sondern auch konsequent angewendet werden. Porphyrius wird aufgefordert, die Kirchen und Versammlungsgebäude der Häretiker zu konfiszieren, den heidnischen Tempeln die Einkünfte zu entziehen, die Götterbilder zu entfernen, die Altäre zu zerstören und die Begräbnisriten zu verbieten. Die örtlichen Bischöfe erhalten Vollmachten zur Unterbindung paganer Riten, und der Kaiser schickt drei agentes in rebus, die die Umsetzung dieser Maßnahmen kontrollieren sollen.

Anlass für dieses Gesetz waren Auseinandersetzungen der Donatisten und der Heiden mit einigen orthodoxen Gemeinden in Africa. Ein allgemeines Konzil der kirchlichen Diözese Africa hatte deshalb am 13. Juni 407 in Carthago beschlossen, die Bischöfe Venantius und Fortunatianus als Gesandte nach Rom adversus Donatistas et paganos zu schicken. ${ }^{3}$ Sie kehrten mit einem Schreiben vom 15. November 407 an den Prokonsul Porphyrius zurück, in dem die Frage des Übertritts zum nizänischen Bekenntnis und das Vorgehen gegen hartnäckige Donatisten geregelt wurden. ${ }^{4}$ Zehn Tage später ist - wohl noch im selben Zusammenhang - das genannte Gesetz für den Prätorianerpräfekten Curtius ausgestellt, doch erst sieben Monate danach in Carthago angeschlagen worden. Dieser Zeitraum lässt sich nicht mit der Entfernung Carthagos von Rom erklären; auch war der Prätorianerpräfekt angewiesen worden, das kaiserliche Gesetz seinen Statthaltern weiterzuleiten..$^{5}$ Allerdings waren am 1. Juni 408 in Calama westlich von Carthago Unruhen nach einer heidnischen Prozession ausgebrochen: Als der nizänische Klerus die Zeremonie verhindern wollte, wurde die Kirche, in der sich die Gläubigen versammelt hatten, gesteinigt und in Brand gesteckt; Bischof Possidius musste sich verstecken und fliehen. Die örtlichen Behörden schritten nicht dagegen ein. ${ }^{6}$ Es ist nicht von der Hand zu weisen, dass der Prokonsul fünf Tage später das Gesetz in Reaktion auf diesen Aufruhr hatte anschlagen lassen. Am 16. Juni trat dann wiederum ein Konzil in Carthago zusammen, das sich ausschließ-

rische und diskriminierende Ausschluss so vieler sectae diente selbstverständlich dazu, die catholica secta zu profilieren.

3 Reg. eccl. Carth. exc. 12,106: placuit et illud, ut adversus Donatistas et paganos vel eorum superstitiones legati missi de hoc glorioso concilio (die Legaten sind 12,97 genannt), quidquid utile perviderint, de gloriosissimis principibus impetrent (MUNIER 1974, 219 und 215).

4 Cod. Theod. 16,5,41.

5 Const. Sirmond. 12: quod ad continendos hominum mores religionemque provisum et ad rectores provinciae sublimis magnificentia tua faciet pervenire et digno per omnes iubebit vigore servari.

6 Zu den Vorgängen Aug. ep. 91,8; vgl. ep. 90; 103; 104 (Briefwechsel mit dem örtlichen Notabeln Nectarius, der Augustinus um Vermittlung bei der zu erwartenden Strafverfolgung wegen seditio bat). Vgl. - freilich für den Osten erlassen - Cod. Theod. 16,4 [de his, qui super religione contendunt], 1 (a. 386; an den praefectus praetorio per Orientem): Diejenigen, die turbulentum quippiam contra nostrae tranquillitatis praeceptum faciendum esse temptaverint, ut seditionis auctores pacisque turbatae ecclesiae, maiestatis capite ac sanguine sint supplicia luituri. 
lich mit diesen Unruhen befasste und eine Gesandtschaft unter Bischof Fortunatianus an den Kaiser in Ravenna contra paganos et haereticos sandte. ${ }^{7}$

Doch war damit noch keineswegs Ruhe eingekehrt: Als der starke Mann des Westens, der magister utriusque militiae Flavius Stilicho, im August 408 ermordet wurde, ${ }^{8}$ gingen donatistische Gemeinden erneut gegen orthodoxe Bischöfe vor, ${ }^{9}$ offenbar weil sie glaubten, die repressiven Religionsgesetze der letzten Jahre seien ein Werk des Stilicho und nicht des Kaisers Honorius gewesen. ${ }^{10}$ Darin bestärkt wurden sie durch gefälschte Kaisergesetze, die religiöse „Toleranz“ (indulgentia) propagierten. ${ }^{11}$ Es kam dabei zu Gewalttätigkeiten gegenüber Klerikern des nizänischen Bekenntnisses und zu gezielten öffentlichen Demütigungen wie der Zurschaustellung des geschorenen Bischofs vor dem Volk. ${ }^{12}$ Die örtlichen Behörden und der Prokonsul unternahmen nichts, weshalb am 13. Oktober wieder ein Konzil in Carthago zusammentrat, das die Bischöfe Restitutus und Florentius nach Ravenna sandte contra paganos et haereticos. ${ }^{13}$ Dieses Mal reagierte der Kaiser schärfer: Nicht nur war der Nachfolger Stilichos, der magister officiorum Olympius, ein überzeugter Vertreter der catholica fides; ${ }^{14}$ besonders der neue Prokonsul Donatus, ${ }^{15}$ dem Honorius ein hartes Vorgehen gegen alle Gegner der catholica secta auftrug, ${ }^{16}$ ging derart eifrig zu Werke, dass ihn Augustinus bat, die Donatisten nicht hinrichten zu lassen, sondern mit geeigneten Zwangsmaßnahmen der nizänischen Kirche zuzuführen. ${ }^{17}$ Vor allem aber übersandte Honori-

7 Reg. eccl. Carth. exc. 13 (MUNIER 1974, 219).

8 PLRE I 853-858 (magister militum von 394-408).

9 Aug. ep. 105,2,3-4 (408 an die Donatisten); Reg. eccl. Carth. exc. 14,106 (MuNIER 1974, 219; Konzil in Carthago am 13. Oktober 408).

10 Aug. ep. 97,2 (408 an den magister officiorum Olympius; PLRE II 801-802, Olympius 2).

11 Aug. ep. 105,3,6: quid est melius, proferre veras imperatorum iussiones pro unitate, an falsas indulgentias pro perversitate: quod vos fecistis, et mendacio vestro subito totam Africam implestis?

12 Const. Sirmond. 14 (Ravenna, 15. Januar 409). Exzerpte aus dieser Konstitution wurden aufgenommen unter Cod. Theod. 16, 2 [de episcopis, ecclesiis et clericis], 31 (= Cod. Iust. I 3 [de episcopis et clericis], 10) und 16,10,5,46 (hier das korrekte Datum).

13 MunIER 1974, 219. Auch Augustinus hatte ein commonitorium über diese Vorgänge nach Italien geschickt (ep. 97,4).

14 Aug. ep. 97,1 (an Olympius): te veraciter filium esse gaudemus. Cod. Theod. 16, 5,42 (Ravenna, 14. November 408) ist an ihn gerichtet.

15 PLRE II 375-376, Donatus 1.

16 Cod. Theod. 16,5,44 (Ravenna, 24. November 408): have, Donate, karissime nobis. Donatistarum haereticorum Iudaeorum nova adque inusitata detexit audacia, quod catholicae fidei velint sacramenta turbare. quae pestis cave contagione latius emanet ac profluat. in eos igitur, qui aliquid, quod sit catholicae sectae contrarium adversumque, temptaverint, supplicium iustae animadversionis expromi praecipimus.

17 Aug. ep. 100 (an Donatus): unde ex occasione terribilium iudicum ac legum, ne in aeterni iudicii poenas incidant, corrigi eos cupimus, non necari; nec disciplinam circa eos neglegi volumus nec supplicia, quae digna sunt, exerceri. sic ergo eorum peccata compesce, ut sint quos paeniteat pecavisse. Augustinus lobte später die Amtsführung des Donatus uneingeschränkt (ep. 112 an Donatus). 
us am 15. Januar 409 seinem neuen, gleichfalls orthodoxen Prätorianerpräfekten Theodorus ${ }^{18}$ ein Gesetz, in dem er zunächst auf die Ereignisse in Africa einging, von denen er nur durch die Bischöfe, nicht aber durch seine Statthalter unterrichtet worden war. Er fügte dem ein allgemeines Edikt gegen Häretiker, Heiden und Juden hinzu, das überall im westlichen Reichsteil bekannt gemacht werden sollte. ${ }^{19}$ Die Konstitution beginnt mit den Worten: ${ }^{20}$

Es besteht kein Zweifel, dass mit dem Einverständnis der Statthalter (coniventia iudicum) geschehen und durch schuldhaftes Wegsehen (dissimulatio) ungeahndet geblieben ist, was zur Störung der öffentlichen Ordnung und zur Verachtung der christlichen Religion, die wir auf geziemende Weise verehren, in aller Öffentlichkeit - wie wir erfahren haben - ausgeführt und zugleich nicht bestraft wurde. Wissentliches Wegsehen liegt nahe am Vergehen bei einem Statthalter, der sich nicht mit der Unkenntnis des vorgefallenen Verbrechens entschuldigen kann. [...] Wir glauben nicht, dass solche Verbrechen den Statthaltern unbekannt bleiben konnten, weil berichtet wurde, dass sie öffentlich in den Städten ausgeübt wurden, und weil die beständige Aufsicht der Magistrate und Kurien sowie die besorgte Aufmerksamkeit ihres Gehilfen, des stationarius, welche Dienerin der Nachrichten und Beweise ist, diese Verbrechen der Abwesenheit der Machthaber zur Kenntnis bringt.

Mit diesen beiden vollständig überlieferten Konstitutionen aus den Jahren 407 und 409 werden - für uns zum ersten Mal erkennbar - kaiserliche und munizipale Amtsträger direkt für die religiösen Konflikte in den Provinzen mitverantwortlich gemacht. Ihnen wird vorgeworfen, mit den Gegnern der kaiserlichen Religionspolitik gemeinsame Sache gemacht oder doch zumindest über die Angriffe auf die nizänische Kirche hinweggesehen zu haben. Freilich scheint das inkriminierte Verhalten der Magistrate gängige Praxis bereits im 4. Jahrhundert gewesen zu sein: Zwar enthalten die Konstitutionen im Codex Theodosianus und im Codex Iustinianus lediglich allgemeine Sanktionsdrohungen für untätige Amtsträger, doch sind bekanntlich ein Großteil der begründenden, rhetorischen Passagen in

18 Flavius Mallius Theodorus ist zum ersten Mal als Prätorianerpräfekt für Italien bezeugt zwischen dem 31. Januar 397 und dem 20. Januar 399; zum zweiten Mal zwischen dem 13. September 408 und dem 15. Januar 409. Er war 396 auch proconsul Africae gewesen (PLRE II 10861087, Theodorus 9).

19 Const. Sirmond. 14: si quidem praesentis legis aeternitate cunctis observanda constituimus Theodore parens carissime atque amantissime, quod illustris magnificentiae tuae praelatum litteris, proponendum edictis, in omnium volumus notitiam pervenire.

20 Const. Sirmond. 14: dubium non est coniventia iudicum fieri et culpabili dissimulatione inultum relinqui quod ad turbandam quietem publicam in contemptum christianae religionis, quam debito cultu veneramur, sub publica testificatione commissum addiscimus et pariter non punitum. vicina peccato in iudice dissimulatio est, quem ignoratio commissi criminis non defendit. [...] ignorari ab his potuisse non credimus quod commissum in civitatibus publice memoratur, quod iugis et magistratuum et ordinum cura, stationarii apparitoris sollicitudo, quae ministra est nuntiorum atque indicium, absentiae exhibet potestatum. 
den Gesetzen der Redaktion der Codices zum Opfer gefallen. ${ }^{21}$ Es stellt sich also die Frage, ob der von kaiserlicher Seite geäußerte Vorwurf der mala desidia iudicum zutrifft, und wenn dem so ist, wie sich ein solches Verhalten erklären lässt.

Die Skizze der religiösen Auseinandersetzungen in Africa ${ }^{22}$ zeigt auch, über welche Zeugnisse wir für die Rolle der Amtsträger bei der Christianisierung der römischen Provinzen typischerweise verfügen: kaiserliche Konstitutionen einerseits und historiographische Texte überwiegend christlicher Provenienz andererseits. Damit stellt sich das Problem, wie sich die unterschiedlichen Perspektiven der normativen und der erzählenden Quellen miteinander verbinden lassen: Waren die zum Teil mit drastischen Sanktionen bewehrten Gesetze so wirkungslos, wie dies ihre dauernde Wiederholung, die Strafandrohungen für die ausführenden Magistrate sowie einzelne Berichte über lokale Ereignisse in den erzählenden Quellen nahe zu legen scheinen? Anders gefragt: Vor welchen Problemen standen die Statthalter, wenn sie die kaiserlichen Direktiven umzusetzen hatten? Dies wirft zudem die Frage auf, welche Verbindlichkeit den kaiserlichen Konstitutionen zukam, wenn sie offenbar auch missachtet werden konnten.

Die gegenüber der Principatszeit dürftige Quellenlage für das Wirken der Statthalter, besonders in religiösen Angelegenheiten, gilt noch verstärkt für die hier interessierende Frage nach der Rolle der Gouverneure bei der Unterdrückung der paganen Kulte: Weitaus mehr Rechtsquellen und erzählende Berichte befassen sich mit den innerkirchlichen Auseinandersetzungen als mit den Konflikten zwischen Heiden und Christen. Da aber seit den 390er Jahren im Zuge der forcierten Formierung der nizänischen Orthodoxie ${ }^{23}$ eine nivellierende Ausgrenzung aller heterodoxen und nichtchristlichen Bekenntnisse zu beobachten ist - auch die eingangs zitierten sirmondianischen Konstitutionen wenden sich gegen Heiden, Juden und Häretiker gleichermaßen -, lässt sich das Verhalten der Gouverneure gegenüber den Heiden wenigstens in Analogie zu ihrer Rolle in den Auseinandersetzungen mit Häretikern oder Juden erhellen.

21 Man vergleiche nur diese beiden sirmondianischen Konstitutionen mit ihren Exzerpten im Codex Theodosianus.

22 Dazu allgemein BROWN 1963; zu den administrativen Strukturen in Africa LEPELLEY 2002.

23 Seit der Erklärung der Nicena fides als alleinige Grundlage der catholica religio im Jahr 380 (Cod. Theod. 16,1 [de fide catholica] 2 (zum Anlass und zur zunächst begrenzten Reichweite dieser Konstitution ERRINGTON 1997, 411-416); 16,5,6,1-2) verdichtet sich das catholicae religionis dogma (Cod. Theod. 16,10,13, a. 395) zur Vorstellung einer sacrosancta lex (Cod. The$o d .16,5,56$, a. 415), die alle Andersgläubigen als auszumerzende Feinde begreift: adversarios catholicae fidei exstirpare huius decreti auctoritate prospeximus (16,6,4 pr.; a. 405); vgl. Cod. Theod. 16,5,63 (a. 423): omnes haereses omnesque perfidias, omnia schismata superstitionesque gentilium, omnes catholicae legi inimicos insectamur errores. Seither ist das öffentliche Wohl, für das der Kaiser zu sorgen hat, nicht mehr vom nizänischen Bekenntnis zu trennen: $s i$ quis contra ea, quae multipliciter pro salute communi, hoc est pro utilitatibus catholicae sacrosanctae ecclesiae adversos haereticos [...] constituta sint (Cod. Theod. 16,5,56; a. 415). Deshalb richten sich häretische und heidnische Kultpraktiken zugleich ad supernae maiestatis iniuriam et temporis nostri contemptum (Nov. Theod. 3,3; a. 438). 
Dieser Quellenbefund ist schon für sich betrachtet signifikant, dokumentiert er doch, wo die hauptsächlichen Konflikte lagen - freilich nur aus kaiserlicher und christlicher Perspektive. Und er spiegelt die relative Bedeutungslosigkeit der Gouverneure für den Christianisierungsprozess wider. Meine These lautet deshalb zugespitzt: Die Statthalter haben bei der Unterdrückung der paganen Kulte keine aktive Rolle gespielt, doch haben sie die zivil- und strafrechtlichen Folgen der Christianisierungsstrategien umsetzen müssen. Auf Grund ihrer Position in der provinzialen Figuration taten sie dies freilich eher schlecht als recht. ${ }^{24}$ Ich werde im Folgenden erstens die kaiserlichen Vorschriften für eine provinziale Religionspolitik untersuchen, wie sie die Codices und Novellen von den Gouverneuren fordern (II), zweitens die Rolle der Statthalter und anderer kaiserlicher Amtsträger bei den gewaltsamen Aktionen gegen pagane Kulte bestimmen, wie sie in der literarischen Überlieferung dargestellt wird (III). Drittens skizziere ich die provinziale Figuration, in der die Gouverneure die kaiserliche Politik durchzusetzen hatten. Daraus ergibt sich eine Antwort auf die Frage, ob und gegebenenfalls weshalb bei der Unterdrückung paganer Kulte tatsächlich von mala desidia iudicum die Rede sein kann (IV).

\section{Die provinziale Religionspolitik gegen ,Heiden` im Spiegel der Konstitutionen}

Die kaiserliche Religionspolitik, wie sie die Konstitutionen spiegeln, betrifft die Christianisierung der Provinzialbevölkerung, die seit Theodosius I. eine forcierte Christianisierung im orthodoxen Sinne war. Der Begriff der Christianisierung, das heißt der Übertritt zur ecclesia catholica, ist in den Rechtsquellen negativ bestimmt und meint in Bezug auf die Heiden ${ }^{25}$ das Verbot paganer Kultpraktiken, die Entfernung und Zerstörung der Altäre und Götterbilder, die Schließung und Zerstörung von Tempeln und Schreinen, den Entzug öffentlicher Mittel für die Heiligtümer und die Beseitigung der Privilegien für heidnische Priester. Es geht also im Wesentlichen um die Zerstörung der materiellen Grundlagen der paganen Kulte (omnis superstitionis materia) ${ }^{26}$ und die soziale Ächtung ihrer Vertreter. Das eigentliche Problem einer Christianisierung, das hier nicht Thema sein kann, hat Augustinus klar gesehen, als er um das Jahr 400 an die heidnischen Kurialen von Madaura schrieb: ${ }^{27}$ contra idola facilius templa quam corda clauduntur, vel potius quae idola non magis in templis quam in vestris cordibus includuntur. Die

24 Zum Verhältnis von Person, gesellschaftlicher Position und politischer Figuration ELIAS 1975, bes. 34-56. Die Figuration ist ein gesellschaftlich determiniertes Beziehungsgeflecht in einem begrenzten politischen Raum. Die gesellschaftlichen Positionen in diesem Beziehungsgeflecht sind weitgehend vorgegeben und von einzelnen Personen nur wenig zu beeinflussen.

26 Cod. Theod. 16,10,16 (a. 399).

27 Aug. ep. 232,1. 
Zerstörung der idola auch in den Herzen war nicht mehr die Aufgabe der Statthalter, sondern der Bischöfe.

Sieht man die einschlägigen Tituli im 16. Buch des Codex Theodosianus und die zeitgenössischen Novellen durch, so lässt sich kein Unterschied des statthalterlichen Aufgabenfeldes (officium) bei der Durchsetzung der kaiserlichen Konstitutionen gegenüber Heiden, Juden, Häretikern und Manichäern ${ }^{28}$ feststellen. Seit dem Ende des 4. Jahrhunderts werden diese sectae häufiger gemeinsam genannt oder Vorschriften, die zunächst für eine von ihnen erlassen wurden, auf die anderen übertragen. Dabei ist zu beobachten, dass den Häretikergesetzen eine Leitfunktion zukam, die in dem Moment Wirksamkeit entfaltete, in dem in den 390er Jahren alle heterodoxen und nichtchristlichen Bekenntnisse zunehmend über einen Leisten geschlagen wurden (als superstitio, error oder insania), da sie die Einheit der nizänischen Kirche zu gefährden schienen. ${ }^{29}$ Waren die schärfsten Zwangsinstrumente zunächst für die Manichäer und bestimmte häretische Bewegungen geschmiedet worden, so ließen sich diese im Zuge einer Verschärfung der Religionskonflikte nach und nach gegen die Donatisten, ${ }^{30}$ die Heiden und schließlich auch gegen die Juden einsetzen, was dazu führte, dass seit den 420er Jahren alle Bekenntnisse, die nicht der Nicena fides verpflichtet waren, gleichermaßen diskriminiert wurden (mit Ausnahme gewisser Privilegien für die Juden). Diese Differenzierung der Maßnahmen gegenüber den unorthodoxen Bekenntnissen erlaubt es aber zugleich festzustellen, welche bereitstehenden Instrumente zur Durchsetzung der kaiserlichen Religionspolitik (noch) nicht auf bestimmte Gruppierungen angewendet wurden.

Wir können uns demnach auf den Titulus XVI 10 (de paganis, sacrificiis et templis) mit den entsprechenden Novellen konzentrieren und Analogien zur bes-

28 Gesondert von den Häretikern werden in den Gesetzen meistens die Manichäer behandelt, die bereits von Diocletian scharf verfolgt wurden (Coll. 15,3,1-8 [Cod. Greg. 7: de maleficis et Manichaeis], a. 297, an den proconsul Africae; zu den Manichäergesetzen seit Diocletian KADEN 1953; FÖGEN 1997, 26-34) und am niedrigsten angesehen waren: qui ad imam usque scelerum nequitiam pervenerunt Manichaei (Cod. Theod. 16,5,65,2; a. 428). Die Konstitutionen kennen drei Klassen von Häretikern, die nach der Schwere ihrer Verfehlung abgestuft werden $(\mathrm{Cod}$. Theod. $16,5,65 ;$ a. 423).

29 Etwa Augustinus (serm. 62,18; a. 399): sciatis autem, charissimi, murmura illorum (paganorum) coniungere se cum haereticis, cum Iudaeis. haeretici, Iudaei et pagani unitatem fecerunt contra unitatem. Vgl. Cod. Theod. 16,10,13 pr. (a. 395): quae olim constituta sunt vel de haereticis vel de paganis; vgl. 16,5,43 (a. $407=$ Const. Sirmond. 12): Donatisten, Montanisten, Manichäer, Priscillianisten, Heiden; 16,5,44 (a. 408): Donatisten, Häretiker, Juden; 16,5,46 (a. $409=$ Const. Sirmond. 14): Häretiker, Juden, Heiden; 16, 8 [de Iudaeis, Coelicolis et Samaritanis], 26 (a. 423): Heiden, Juden, Häretiker; Nov. Theod. 3,2 (a. 438) richtet sich gegen die ferales sectae der Juden, Samaritaner, Heiden und Häretiker. Alle seien supernae maiestati et Romanis legibus inimici. - Die Tendenz der Konstitutionen, alle Bekenntnisse, die nicht dem nizänischen Christentum (vera religio) entsprechen, gemeinsam als superstitio zu behandeln, lässt sich seit 395 beobachten; dazu SALZMAN 1987, 182-183.

30 Die Donatisten - zunächst als Schismatiker betrachtet - wurden im Jahr 405 zu Häretikern erklärt: ita contigit, ut haeresis ex schismate nasceretur ( $\mathrm{Cod}$. Theod. 16,6 [ne sanctum baptisma iteretur], 4 pr.; vgl. 16,5,38-39). 
ser dokumentierten Häretikergesetzgebung ziehen (Titulus XVI 5: de haereticis). Die 25 Konstitutionen aus den Jahren 320 bis $435^{31}$ sind zumeist an die Prätorianerpräfekten, die Stadtpräfekten oder an die dem Kaiser direkt unterstellten Prokonsuln gerichtet, ${ }^{32}$ zuweilen auch an Vikare ${ }^{33}$ und militärische Befehlshaber (comites und duces), ${ }^{34}$ kaum je aber an die ordinarii iudices, das heißt an die consulares, praesides oder correctores selbst. ${ }^{35}$ In ihnen wird in dreifacher Weise auf die Gouverneure Bezug genommen: Erstens enthalten einige Konstitutionen Bestimmungen über die Kultpraxis und die Religionszugehörigkeit der Statthalter selbst, denen Strafen angedroht werden, sollten sie die inkriminierten Riten praktizieren. Zweitens haben diese dafür zu sorgen, dass die Anordnungen mit einem begleitenden Edikt in der Provinz publik gemacht und dass sie von den officiales des Statthalterbüros sowie den Kurialen durchgesetzt werden. Drittens schließlich werden die Gouverneure und ihre officia mit Sanktionen bedroht, sollten sie dabei nachlässig sein oder gar in geheimem Einverständnis mit den Delinquenten stehen.

1. Die christlichen Kaiser konnten bei der Ernennung von Gouverneuren auf deren Religionszugehörigkeit ${ }^{36}$ Einfluss nehmen. Dies taten etwa Constantin ${ }^{37}$ oder - wie eingangs dargelegt - Honorius, als er in der zweiten Hälfte des Jahres 408 rechtgläubige Amtsträger wie den Prokonsul Donatus bestallte. Umgekehrt wird von Kaiser Julian berichtet, dass Christen unter seiner Herrschaft nicht Statthalter werden durften. ${ }^{38}$ Neben dieser informellen Einflussnahme versuchten die christlichen Kaiser von Anfang an, ihren Amtsträgern bestimmte altgläubige Kultpraktiken zu untersagen. So erließ Constantin im Jahr 324 ein Opferverbot für Statthalter und höhere Amtsträger - ich halte diese in der Forschung umstrittene Nachricht von Eusebios vor dem Hintergrund der Bedeutung des Opferns wäh-

31 Die Konstitutionen sind zum Teil aufgenommen in Cod. Iust. 1,11 [de paganis sacrificiis et templis]; zu den Gesetzen von Constantin BRADBURY 1994, von Constantius II. LEPPIN 1999, 466-475 und denjenigen der theodosianischen Dynastie TROMBLEY 1993, I 10-35; 72-94; vgl. CURRAN 1999, 159-217.

32 Cod. Theod. $16,10,17$ und 18 (a. 399).

33 Cod. Theod. 16,10,15 (a. 399).

34 Cod. Theod. 16,10,8 (a. 364); 16,10,11 (a. 391).

35 Die Konstitutionen unterscheiden deutlich zwischen proconsules und ordinarii iudices (consulares, praesides, correctores), vgl. etwa Cod. Iust. 1,54 [de modo multarum, quae ab iudicibus infliguntur], 6 (a. 399); JONES 1964, 373-377. Die praesides und correctores erhielten gegen Ende des 4. Jahrhunderts ebenfalls den senatorischen Rang von viri clarissimi, den die consulares bereits besaßen. Die Prokonsuln standen im Rang von viri spectabiles.

$36 \mathrm{Zu}$ den Problemen einer statistischen Bestimmung der Religionszugehörigkeit von höheren Amtsträgern BARNES 1995, der sich mit den früheren Untersuchungen - besonders der Arbeit Rabans von Haehling - auseinandersetzt. Für die Statthalter sind diese Untersuchungen weitgehend irrelevant, da sie bestenfalls die Prokonsuln miteinbeziehen, sich im übrigen aber auf die besser dokumentierten Konsuln, Prätorianerpräfekten und Stadtpräfekten konzentrieren.

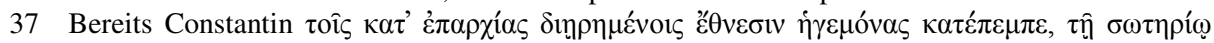

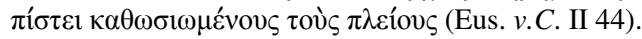

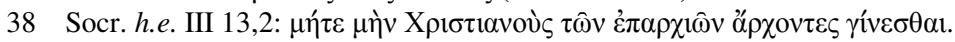


rend der Christenverfolgungen für glaubwürdig. ${ }^{39}$ Obwohl sich die Kultbeschränkungen und Kultverbote in den Konstitutionen der folgenden Zeit prinzipiell auf alle Reichsbewohner bezogen ${ }^{40}$ scheint das früheste Gesetz, das die pagane Kultpraxis speziell der Gouverneure ahndete, erst unter Theodosius I. im Jahr 391 erlassen worden zu sein. Es untersagte den Statthaltern, auf ihren Reisen Tempel aufzusuchen, um dort Götterbilder zu verehren und profani ritus zu vollziehen.

Andererseits aber werden sich die Gouverneure nur selten offen gegen die kaiserliche Religionspolitik gestellt und sich als Anhänger des traditionellen Glaubens profiliert haben, zumal wenn sie eine weitere Karriere im Reichsdienst anstrebten. War jenes dennoch der Fall, wurde es ausdrücklich vermerkt, besonders wenn pagane Schriftsteller ein Zeugnis heidnischer Standfestigkeit geben wollten. So überliefert Zosimos, wie der proconsul Achaiae Vettius Agorius Praetextatus im Jahr 364 das zuvor von Valentinian I. erlassene Verbot nächtlicher Zeremonien für seine Provinz unter Hinweis auf die Bedeutung der Mysterien für Athen außer Kraft setzen konnte, indem er ein kaiserliches privilegium erwirkte. ${ }^{42}$

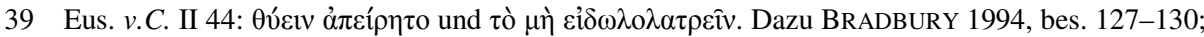
CURRAN 1999, 172-178. Constantin untersagte im Jahr 333 in seinem Reskript für die Stadt Hispellum das Opfern im Rahmen des Kaiserkultes: ea observatione perscripta ne aedis nostro nomini dedicata cuiusquam contagiose superstitionis fraudibus polluatur (CIL 11,5265 = ILS 705, Z. 45-48; zur Bedeutung von superstitio in dieser Inschrift SAlZMANN 1987, 178). Auch für das Heiligtum von Mamre in Palaestina verfügte Constantin ein Opferverbot (Eus. v.C. 3,52-53; Soz. h.e. 2,4). Auf ein allgemeines Verbot des sacrificium durch eine lex des Constantin bezog sich im Jahr 341 Constans (Cod. Theod. 16,10,2; die intitulatio nennt Constantius, doch ist die Konstitution für den Westen ausgestellt); jenes ist jedoch nicht erhalten. Noch im Jahr 320 hatte Constantin verfügt, dass nach Blitzeinschlägen im Palast und in öffentlichen Gebäuden die Haruspices gemäß dem traditionellen Ritus (und das heißt mit einem Opfer) Ursachenforschung betreiben dürften. Er verbot nur die private Haruspicin (domestica sacrificia; Cod. Theod. 16,10,1 pr.; vgl. Cod. Theod. 9,16 [de malificis et mathematicis] 2; a. 319; dazu FÖGEN 1997, 34-39; 255-257).

40 Normalerweise bezogen sich die Gesetze pauschal auf alle Personen, die pagane Riten praktizieren: qui ... oder si quis ..., während in Bezug auf die häretischen Bewegungen meistens zwischen Bischöfen, Klerikern und Gläubigen unterschieden wurde. Theodosius präzisierte dieses qui in einer Konstitution des Jahres 392 (Cod. Theod. 16,10,12 pr.): nullus omnino ex quolibet genere ordine hominum dignitatum vel in potestate positus vel honore perfunctus, sive potens sorte nascendi seu humilis genere condicione fortuna.

41 Cod. Theod. 16,10,10. Die Strafsumme für Statthalter im Rang von correctores und praesides betrug 4 Pfund Gold (288 Solidi), die von consulares 6 Pfund Gold (432 Solidi), die der höherrangigen iudices, das heißt der proconsules, 15 Pfund Gold (1080 Solidi). Eine entsprechende Mult mussten ihre jeweiligen officia bezahlen. Aus demselben Jahr stammt die Strafandrohung für praesides, die unter Ausnutzung ihrer Amtsstellung (fretus privilegio potestatis) Tempel aufsuchten (Cod. Theod. 16,10,11; a. 391). - Der Begriff profanus ritus zeigt an, dass die paganen Kulthandlungen aus christlicher Sicht nichts mit der göttlichen Sphäre zu tun haben. Der häretische Kult wird als sacrilegus ritus bezeichnet (Cod. Theod. 16,10 $19=$ Cod. Iust. I 11,5; a. 407).

42 Zos. 4,3,3; PLRE I 722-724, Praetextatus 1; Cod. Theod. 9,16 [de maleficiis et mathematicis], 7 (a. 364): ne quis deinceps nocturnis temporibus aut nefarias preces aut magicos apparatus aut sacrificia funesta celebrare conetur. Ähnlich schon im Jahr 353 (Cod. Theod. 16,10,5). 
Und Eunapios berichtet, dass etwa 15 Jahre später der vicarius Asiae Iustus und der consularis Lydiae Hilarius gemeinsam in Sardeis verfallene Altäre und Heiligtümer restaurierten und öffentlich Opfer darbrachten. ${ }^{43}$ Solche demonstrativen Gesten waren bemerkenswert und wurden deshalb aufgezeichnet und überliefert. Doch fällt auf, dass die meisten dieser Nachrichten aus vortheodosianischer Zeit stammen, als die Konflikte mit Donatisten und Arianern unter Constans und Constantius II., die religionspolitische Wende unter Julian und die erneuten Auseinandersetzungen um die Orthodoxie unter Valens und Valentinian I. polarisierten und zugleich die kaiserliche Macht schwächten. Wie zu Beginn des 5. Jahrhunderts in Africa profitierten oder litten dabei die religiösen Parteiungen im Gefolge der politischen Kämpfe.

Es vergingen weitere 25 Jahre, ehe Theodosius II. im Jahr 416 im östlichen Reichsteil ein Gesetz erließ, das explizit den Heiden das Statthalteramt verwehrte. ${ }^{44}$ Dennoch sind für die erste Hälfte des 5. Jahrhunderts weiterhin pagane Gouverneure bezeugt, etwa der praeses Cariae Dulcitius, der sich in einer Inschrift aus Aphrodisias als Agonothet und Maiumarchēs ehren ließ, obwohl das Maiumas-Fest im Jahr 399 endgültig verboten worden war, ${ }^{45}$ oder einige praesides

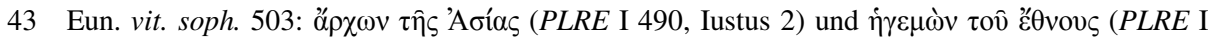
435, Hilarius 10). Auch der praefectus praetorio per Illyricum Anatolius (357-360; PLRE I 5960, Anatolius 3) opferte unter Constantius II. „,mutig“ bei seinem Aufenthalt in Athen und besuchte dort die Tempel (Eun. vit. soph. 491). Und der vicarius Asiae (354-359) Flavius Magnus (PLRE I 535, Magnus 9) errichtete in Hierapolis (Phrygia Pacatiana) ein Temenos für die Nymphen und stiftete ihnen ein Fest (SEG 36, 1986, 1198 = MERKELBACH - STAUBER 1998, 266268). Vielleicht spielt die Formulierung, ,der die Städte durch seine Rechtsprechung gerettet hat", in einer Ehreninschrift für den heidnischen praefectus praetorio Orientis Flavius Eutolmius Tatianus (388-392; PLRE I 876-878, Tatianus 5) darauf an, dass dieser eine konsequente Umsetzung der theodosianischen Religionspolitik verhindert hatte (ROUECHÉ 1989, 63-66 = MERKELBACH - StAuber 1998, 246).

44 Cod. Theod. 16,10,21: qui profano pagani ritus errore seu crimine polluuntur, hoc est gentiles, nec ad militiam admittantur nec administratoris vel iudicis honore decorentur; vgl. Zos. 5,46. Die im Jahr 408 im Westen verfügte Konstitution (Cod. Theod. 16,5,42: eos qui catholicae sectae sunt inimici, intra palatium militare prohibemus) bezieht sich nur auf den Hofdienst, nicht die Reichsverwaltung (zum Unterschied von intra palatium und administratio vgl. Cod. Theod. 6,22 [de honorariis codicillis], 1; a. 321 und 6,22,7,1; a. 383). - Häretikern war bereits 395 die militandi facultas verwehrt worden (Cod. Theod. 16,5,25 und 29; vgl. 16,5,58,7 und Const. Sirmond. 6; a. 425; ENSSLIN 1953, 64 geht von einem Verbot schon im Jahr 389 aus). Auch Juden waren seit 404 vom Reichsdienst ausgeschlossen (Cod. Theod. 16,8,16: Iudaeos et Samaritanos [...] omni militia privandos esse censemus; vgl. 16,8,24: Ausschluss aus der militia palatina wie der militia armata; a. 418), doch findet sich noch im Jahr 417 ein aus Minorca stammender jüdischer praeses insularum Balearum, Lectorius (PLRE II 661), der anschließend zum comes aufstieg (Severus von Minorca ep. 24,2; BRADBURY 1996, 116). Zur selben Zeit war der vom Prätorianerpräfekten bestätigte defensor civitatis in Minorca Jude (Severus Menorc. ep. 19,6; BRADBURY 1996, 108), obwohl diese seit 409 nicht mehr defensores werden durften (Cod. Iust. 1,55 [de defensoribus civitatum], 8).

45 Roueché 1989, 73-75 = Merkelbach - Stauber 1998, 236-237. Dulcitius wird in mehreren Inschriften in Aphrodisias als $\dot{\eta} \gamma \varepsilon \mu \omega \dot{v}$ und $\kappa \tau i ́ \sigma \tau\rceil \varsigma$ geehrt, auch von seinem princeps officii ( $\left.\pi \rho \hat{\tau} \tau о \varsigma \sigma \tau \rho \alpha \tau^{\prime} \eta \varsigma\right)$. Dies legt es nahe, in ihm einen praeses Cariae zu sehen. Das Maiumas-Fest 
Thebaidos, mit denen Abt Schenute von Atripe in Konflikt geriet. ${ }^{46}$ Die Konstitution von 416 war das letzte Gesetz, das sich direkt mit der Kultpraxis der Gouverneure befasste; spätere Konstitutionen richteten sich wie auch schon im 4. Jahrhundert gegen Heiden generell. In der ganzen Zeit zwischen 324 und 416 wurden den Gouverneuren also nur für tatsächlich praktizierte profani ritus und nicht für eine Gesinnung Strafen angedroht. Doch hatte sich zwischen 391 und 416 die Sanktion von einer Geldstrafe hin zum Ausschluss aus dem gesamten kaiserlichen Dienst verschärft.

2. Die Durchsetzung der kaiserlichen Religionspolitik war von zwei Faktoren abhängig: den rechtlichen Rahmenbedingungen (wozu auch die Frage nach der Verbindlichkeit der Konstitutionen gehört) und den sozialen Verhältnissen, in denen der Gouverneur wirkte. Hier soll zunächst vom rechtlichen Aspekt die Rede sein.

Welchem religiösen Bekenntnis ein Statthalter auch zuneigte, seine vornehmliche Aufgabe bestand darin, einerseits die „tausend Strafandrohungen“ der kaiserlichen Religionsgesetze (promulgatarum legum mille terrores) ${ }^{47}$ zur Geltung $\mathrm{zu}$ bringen und andererseits darauf zu achten, dass dabei die öffentliche Ordnung (quies publica) gewahrt blieb. Die erste Aufgabe ruft die afrikanische Konstitution vom 15. Januar 409 eindringlich in Erinnerung: $:^{48}$
Alle Statthalter sollen wissen, dass den Vorschriften der (bislang gegen die Häre- tiker, Heiden und Juden) erlassenen Gesetze mit loyaler Hingabe gehorcht wer- den muss, und es gehört zu ihren vordringlichen Pflichten, dass sie nicht zögern das auszuführen, was wir gegen jene verfügt haben.

Wenn dem Gouverneur neue Gesetze durch ein Schreiben des Prätorianerpräfekten zugestellt wurden, bestand seine Pflicht zunächst darin, dafür zu sorgen, dass die örtlichen Machthaber (magistratus, decuriones, defensores civitatis, possessores) in Zusammenarbeit mit den officiales des Statthalterbüros, zu denen auch diejenigen beneficiarii gehörten, die als stationarii über die Provinz verteilt waren, die Vorschriften ausführten. Der Statthalter, der nur für kurze Zeit in der Provinz weilte und in der Regel mit den örtlichen Verhältnissen wenig vertraut (und nicht selten auch inkompetent) war, übte im wesentlichen eine Oberaufsicht, ${ }^{49}$ während seine apparitores, die sich zum Teil aus der Provinz rekrutierten und dort lange Dienst taten, weitgehend selbständig handelten. So schrieb ein

wurde zunächst im Jahr 396 ausdrücklich erlaubt, doch ohne dessen obszöne Riten, dann aber endgültig 399 verboten (Cod. Theod. 15,6 [de Maiuma] 1-2). Zum Maiumas-Fest in Antiocheia HAHN 2004, 134-136.

46 TROMBLEY 1994, II 110-114; HAHN 2004, 241; 254-257.

47 Nov. Theod. 3,8 (a. 438).

48 Const. Sirmond. 14 = Cod. Theod. 16,5,46: noverint iudices universi praeceptis eorum (legum) fideli devotione parendum et inter praecipua curarum quiquid adversus eos decrevimus non ambigant exsequendum.

49 Der Terminus ist custodire: Cod. Theod. 16,10,13,1 (a. 399): custodire omni industria atque cautela; 10,12,4 (a. 392): per iudices volumus [...] custodiri. 
Gesetz vor, dass heidnische Kultbilder nur nach einer Untersuchung und unter Aufsicht der officiales entfernt werden dürften. ${ }^{50}$ Doch bereits die Umsetzung der kaiserlichen Gesetze konnte behindert werden. Dies zeigt ein Brief Kaiser Julians an den praefectus Aegypti Ecdicius Olympus, in dem jener sich über die Missachtung seines Dekretes beklagt, das die Verbannung des Bischofs Athanasios aus Alexandreia verfügt hatte. Doch nicht dem Gouverneur selbst, den er nur tadelte, sondern seinem officium ( $\tau \alpha \dot{\xi} \xi \varsigma$ ) drohte Julian eine empfindliche Geldstrafe an. ${ }^{51}$ So haben manche Amtsträger schon die Publikation des kaiserlichen Gesetzes unterlassen, wenn sie mit dessen Inhalt nicht einverstanden waren wie der praefectus praetorio Orientis Flavius Taurus, der im Jahr 433/34 das kaiserliche Dekret, die Bischöfe Kilikiens sollten die Erhebung des Johannes von Antiocheia zum Bischof anerkennen, nicht publizierte mit der Begründung, der zu erwartende Aufruhr (futuras ex hoc eversiones) würde die Steuereinziehung (tributa solven$d a)$ gefährden. ${ }^{52}$ Und auch der eingangs erwähnte Prokonsul Porphyrius hatte anscheinend gezögert, das kaiserliche Gesetz wie üblich mit einem begleitenden Edikt zu veröffentlichen. ${ }^{53}$

Wie die zitierte Passage aus der 14. sirmondianischen Konstitution zeigt, war es die Aufgabe der lokalen Magistrate und besonders der stationarii, die Delinquenten ausfindig zu machen und an den Statthalter zu überstellen, damit sie vor dem Tribunal angeklagt und verurteilt werden konnten. ${ }^{54}$ Jene hatten auch die

50 Cod. Theod. 16,10,18 (a. 399): depositis sub officio idolis disceptatione habita. Bücherverbrennungen, die die Eunomianer und Montanisten betrafen, fanden dagegen sub aspectibus iudicantum statt, das heißt im Anschluss an die Verurteilung vor dem Tribunal (Cod. Theod. $16,5,34,1 ;$ a. 398).

51 Iul. ep. 112 (ed. Bidez - Cumont); PLRE I 647, Olympus 3.

52 Acta conciliorum oecumenicorum, Bd. IV 1: Collectio Casinensis, 155 (Schwartz); PLRE II 1056-1057, Taurus 4.

53 Das Konsistorium beschloss die kaiserlichen Gesetze, die der quaestor sacrii palatii vorformuliert hatte (leges promulgatae). Anschließend wurden diese ganz oder zu Teilen mit Blick auf den Adressaten umformuliert und in Form einer epistula an den betreffenden Amtsträger geschickt. War dies der praefectus praetorio, so sandte er das Schreiben an die ihm untergebenen Statthalter (leges receptae), die es unter einem eigenen Begleitedikt anschlagen ließen (leges propositae): data VII kal. Decemb. Romae (25. 11. 407), proposita Carthagine in foro sub programmate Porphyrii proconsulis nonis Iuniis Basso et Filippo vv. cc. conss (5. 6. 408; Const. Sirmond. 12); ebenso wurde das Gesetz zur Zerstörung der ländlichen Tempel proposita Damasco (Cod. Theod. 16,10,16; a. 399). Der Gouverneur gab die Konstitution dann an die städtischen Magistrate weiter (Eus. h.e. 8,17,2; 9,1,2; 10,6; Nov. Theod. 3 [de Iudaeis, Samaritanis, haereticis et paganis], 10 (a. 438 an den Prätorianerpräfekten): propositis excellentiae suae sollemniter edictis in omnium faciat pervenire notitiam, provinciarum quoque moderatoribus praecipiat intimari, ut et eorum par sollicitudine cunctis civitatibus atque provinciis quae necessario sanximus innotescant. Zur Verbreitung der kaiserlichen Konstitutionen und zu deren uneinheitlicher Terminologie JONES 1964, 472-474; MATTHEWS 1993, 27; CORCORAN 1996, 245-249; HONORÉ 1998, 135-136; MATTHEWS 2000, 168-199.

54 Cod. Theod. 16,10,12,4 (a. 392): ita per iudices ac defensores et curiales singularum urbium volumus custodiri, ut ilico per hos comperta in iudicium deferantur, per illos delata plectantur. Vgl. Cod. Theod. 16,2,31 (a. 409): quod geritur (sacrilegium) litteris ordinum, magistratuum et 
Beweise für den Prozess beizubringen. ${ }^{55}$ Dies war keineswegs immer einfach, denn die herrschaftliche Durchdringung der Provinz war - dies zeigen die detaillierten Vorschriften zur Verfolgung der Heiden und Häretiker - komplex: Die Unterdrückung und damit die Überwachung der verbotenen Riten bezog sich nicht nur pauschal auf öffentliche und private Räume sowie darauf, ob sie vor aller Augen oder im Geheimen stattfanden. ${ }^{56} \mathrm{Da}$ es um Kultstätten und Versammlungsräume ging, musste auf die herrschaftliche Organisation des provinzialen Territoriums Rücksicht genommen, das heißt auf verschiedene Herren und Magistrate zugegriffen werden. Grundsätzlich wird dabei unterschieden zwischen loca publica in der Verantwortung städtischer Magistrate, kaiserlichen possessiones unter der Aufsicht von Prokuratoren und privaten Grundstïcken der domini. Als zentral erscheint in den Konstitutionen zunächst der Gegensatz von Stadt und Land, ${ }^{57}$ was nicht nur daran liegt, dass bestimmte Städte bald christianisiert waren, während ihre ländlichen Territorien noch pagan waren, ${ }^{58}$ oder umgekehrt, dass eine ländliche Region bereits christianisiert war, während die Stadt noch mehrheitlich heidnisch blieb. ${ }^{59}$ Vielmehr bestand eine häufige Strafmaßnahme darin, Heiden oder Häretiker der Stadt zu verweisen, indem man sie zu exilium bzw. deportatio verurteilte. ${ }^{60}$ Da die Kontrolle in der Stadt dichter war, flohen bei Konflikten Heiden oder Häretiker auch in das umliegende Land.

curatorum et notoriis apparitorum, quos stationarios appellant, deferatur in notitiam potestatum.

55 Act. Agap. 3: Agape und ihre Genossinnen wurden im Jahr 304 غ̇ंì von Macedonia gebracht, weil sie das Kaiseropfer verweigerten. Der commentarius sagte vor

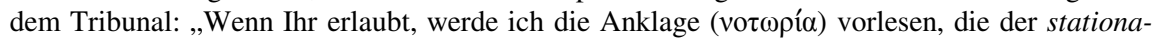
rius betreffs der vor Gericht stehenden Personen Euerem Genius gesandt hat." Vgl. allgemein Cod. Theod. 6,29 [de curiosis], 1 (a. 355): memorati igitur curiosi et stationarii vel quicumque funguntur hoc munere crimina iudicibus nuntianda meminerint et sibi necessitatem probationis incumbere, non citra periculum sui, si insontibus eos calumnias nexuisse constiterit. Der Übergang vom Akkusations- zum Inquisitionsprozess in der Spätantike (SANTALUCIA 1998, 280 283) bedeutete, dass nun die Amtsträger die Verbrechen aufzuspüren und anzuzeigen hatten, was freilich die Denunziation bzw. Anklage durch Privatpersonen nicht überflüssig machte: sive accusator exsistat sive eum (reum) publicae sollicitudinis cura perduxerit, statim debet quaestio fieri (Cod. Theod. 9,3 [de custodia reorum], 1 pr. = Cod. Iust. 9,4,1; a. 320). Der inquisitorische Aspekt der Religionsgesetze zeigt sich in Formulierungen wie: perquisiti ab omnibus urbibus ac locis (Cod. Theod. 16,5,12; a. 383; vgl. 16,5,9,1; a. 382; 16,5,15; a. 388; 16,5,35; a. 399). Aufschlussreich wiederum ist, dass sich die Aufforderung zum Aufspüren auf häretische, nicht aber pagane Delinquenten bezieht.

56 Cod. Theod. 16,5,26 (a. 395): nec publice nec privatim, nec in secreto nec palam; vgl. Cod. Theod. 16,5,9,1 (a. 382); 16,5,12 (a. 383).

57 Cod. Theod. 16,5,4 (a. 376): sive in oppidis sive in agris; vgl. Cod. Theod. 16,5,9,1; 16,5,12: neque publicis neque priviatis aditionibus intra urbium adque agrorum ac villarum loca.

58 Etwa Constantinopel und sein bithynisches Hinterland oder Antiocheia und sein Umland; vgl. TROMBLEY 1994, II 76-95 und 134-150; TROMBLEY 1985.

59 Etwa Panopolis in der Thebais zu Beginn des 5. Jahrhunderts; vgl. HAHN 2004, 242-246.

60 Cod. Theod. 16,5,34 pr. (a. 398): Eunomianae superstitionis clerici seu Montanistae consortio vel conversatione civitatum universarum adque urbium expellantur; vgl. Cod. Theod. 16,5,6,3 (a. 381); 16,5,14 (a. 388). Nur die Manichäer sollten ganz vom römischen Territorium ver- 
In der Stadt waren generell die Kurie und ihre Magistrate für die kultische Disziplin verantwortlich, während die städtischen Korporationen dafür zu sorgen hatten, dass ihre Kollegiaten sich nicht zu verbotenen religiösen conventicula tumultuosa zusammenrotteten. ${ }^{61}$ Außerhalb der Stadt sind die dörflichen Gemeinden und die Ländereien der Großgrundbesitzer als eigene Rechtsräume zu unterscheiden. Während in den Gemeinden die Magistrate für die gewünschte Rechtgläubigkeit sorgen mussten, unterscheiden die Konstitutionen bezüglich des Großgrundbesitzes präzise nach villae dominicae, villae publici iuris und privaten possessiones. Kriterium ist in jedem Fall, ob der Eigentümer, der Pächter oder der Verwalter die verbotenen Riten auf seinem Grundstück wissentlich zugelassen hatte oder ob er davon keine Kenntnis besaß. Je nachdem wurden die privaten Großgrundbesitzer (domini) und die freigeborenen Pächter (conductores) mit Geldstrafen, Konfiskation oder Deportation bedroht, wobei die Schärfe der Strafen auch von der Art des Kultes abhing. Gegen die unfreien oder freigelassenen Pächter und Verwalter (procuratores, vilici, actores) wurde neben Prügelstrafe, Geißelung und Deportation in die Minen auch die Todesstrafe angedroht. Ein besonderes Problem scheinen die kaiserlichen Güter gebildet zu haben, denn hier versagte die Drohung mit der Konfiskation. ${ }^{62}$ Die possessores und conductores wurden also generell für die religiöse Praxis ihrer servi und coloni, über die sie ein Züchtigungsrecht besaßen, verantwortlich gemacht. ${ }^{63}$

Aus diesen präzisen Bestimmungen vor allem der theodosianischen Konstitutionen lässt sich erschließen, dass die eigentlichen Probleme bei der Durchsetzung der kaiserlichen Religionsgesetze im ländlichen Bereich lagen. ${ }^{64}$ Unter dem Schutz der Grundherren oder Prokuratoren konnten noch zu einer Zeit pagane oder heterodoxe Riten praktiziert werden, als dies in vielen Städten schon nicht mehr möglich war. Freilich gab es auch religiöse Konflikte zwischen heidnischen

schwinden (ex omni orbe terrarum; Cod. Theod. 16,5,18; a. 389). Sie stellten sich aus römischchristlicher Sicht außerhalb des ius commune (Cod. Theod. 16,5,40 pr.; a. 407): huic itaque hominum generi nihil ex moribus, nihil ex legibus sit commune cum ceteris.

61 Cod. Theod. 16,4 [his qui super religione contendunt], 5 (a. 404). Als Bischof Eleusios von Kyzikos zur Zeit von Constantius II. Tempel zerstörte und heilige Bezirke entweihte

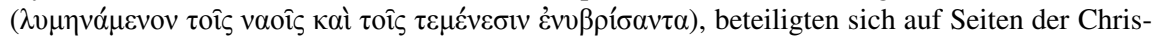
ten lokale Kollegien ( $\tau \alpha ́ \gamma \mu \alpha \tau \alpha)$ der Handwerker. Beiden warf Julian dann vor, um der Religion

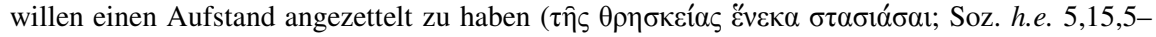
7).

62 Cod. Theod. 16,10,13 pr. (a. 395): possessionum nostrarum, in quibus sine timore dispendii coetus inlicitos haereticos inire conperimus, eo, quod fisco sociari non possunt.

63 Cod. Theod. $16,10,12$ (a. 391); 16,5,21 (a. 392); 16,5,34 pr. (a. 398); 16,5,36,1 (a. 399); 16,5,40,7 (a. 407); 16,5,52 (a. 412). Zu den Straftarifen vgl. bes. Cod. Theod. 16,5,52 und 54 (a. $414)$.

64 Was freilich die Kontrolle der Steuererhebung angeht, sollten sich die praesides ein eigenes Bild von der Arbeit ihrer Steuereinnehmer machen und per omnium villas sensim atque usitatim vicosque cunctos discurrant (Cod. Theod. 1,16 [de officio rectoris provinciae], 11; a. 369). Dies ist umso bemerkenswerter, als die Gouverneure ihr Tribunal nur noch im praetorium der Provinzhauptstadt hielten und keine Konventsorte mehr besuchten (SANTALUCIA 1998, 272-273). 
Grundherren und der von ihnen abhängigen, bereits christianisierten Bevölkerung, wie das Beispiel des ehemaligen praeses Thebaidos und Großgrundbesitzers Flavius Aelius Gessius in der Chora von Panopolis lehrt. ${ }^{6 .}$

Die Ereignisse in Africa zeigen, dass es sich bei den religiösen Auseinandersetzungen aus der Sicht der Kaiser und ihrer Amtsträger um Aufruhr (seditio) handelte, der die quies publica bedrohte. Auf seditio aber stand die Todesstrafe. ${ }^{66}$ Verantwortlich für den Aufruhr waren conventicula tumultuosa, Mönchsbanden und andere Zusammenrottungen, ${ }^{67}$ die von den Protagonisten der religiösen Parteiungen angeführt wurden. Für den Gouverneur stellten diese Konflikte ein Ordnungsproblem dar; er musste den Aufruhr ersticken und die publica disciplina wiederherstellen. Genau dies aber hatte der Prokonsul Porphyrius versäumt, was ihm Honorius vorwarf. ${ }^{68}$

Andererseits hatte ein Statthalter aber auch darauf zu achten, dass er durch seine Maßnahmen nicht selbst Aufruhr stiftete. Dem trug ein Gesetz aus dem Jahr 399 Rechnung, das die Zerstörung der Tempel in ländlichen Gebieten mit der Warnung verfügte, dies solle sine turba ac tumultu geschehen ${ }^{69}$ Ähnlich hieß es im Jahr 415, Synagogen könnten im ländlichen Bereich zerstört werden, si sine seditione possint deponi. ${ }^{70}$ Selbst Kaiser haben derartige Rücksichten genommen, so im Jahr 399 Arcadius, als er der Zerstörung des Marnas-Tempels in Gaza zunächst nicht zustimmte, um die fast gänzlich pagane Bevölkerung der prosperierenden Stadt, die loyal war und reichlich Steuern zahlte, nicht vor den Kopf zu stoßen. ${ }^{71}$ Unter dem Gesichtspunkt des Ausbruchs von Unruhen also war die Durchsetzung einer orthodoxen Religionspolitik in vielen Städten mit einer gemischten Bevölkerung eine heikle Angelegenheit. Und selbst als dies in den meisten Städten des Reiches im Laufe des ersten Drittels des 5. Jahrhunderts zumin-

65 EMmel 2002; HAHN 2004, 237-242; PLRE I 395, Gessius 2. Siehe auch den Beitrag von EMMEL in diesem Band.

66 Deshalb bezeugte Honorius clementia, als er anordnete (Const. Sirmond. 14): si convinci manifesta probatione cognoverit, cuiuslibet dignitatis et honoris reos probatos aut metallo tradat aut poenam deportationis subire conpellat, facultatibus eorum fisco nostro sociatis.

67 Liban. or. 30,9: Mönche in der Chora von Antiocheia; Call. v. Hyp. 30,1: Mönche als Beglei-

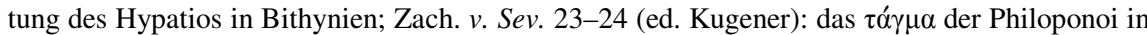
Alexandreia; Soz. h.e. 7,15,12: Dorfbewohner zum Schutz der Tempel in Apameia.

68 Als dagegen die Bevölkerung in Gaza einige Christen lynchte und der consularis Palaestinae daraufhin die Rädelsführer verhaftete, ließ ihn Julian angeblich absetzen (Soz. h.e. 5,9). Den Aufruhr in Alexandreia im Jahr 391 versuchten der comes Aegypti und der praefectus Augustalis zu beenden; als sie seiner nicht Herr werden konnten, schrieben sie an den Kaiser (Soz. h.e. 7,15,7). Und als sich der praefectus Augustalis mit dem Unmut der ägyptischen Bevölkerung konfrontiert sah, weil diese nicht für die Nilflut opfern durfte, befürchtete er einen Aufstand und meldete dies dem Kaiser (Soz. h.e. 7,20,3).

69 Cod. Theod. 16,10,16.

70 Cod. Theod. 16,8 [de Iudaeis, Caelicolis et Samaritanis], 22.

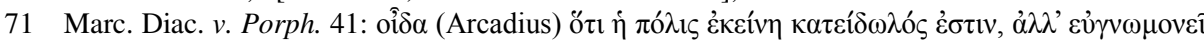

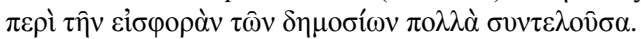


dest oberflächlich gelungen schien, ${ }^{72}$ sahen sich die Kaiser im Jahr 423 nun veranlasst, Christen von Angriffen auf Heiden und Juden abzuhalten, die sich nichts hatten zu Schulden kommen lassen, das heißt keine profani oder sacrilegi ritus praktiziert hatten (Iudaei ac pagani in quiete degentes). ${ }^{73}$

Die kaiserlichen Religionsgesetze wurden gegen Ende des 4. Jahrhunderts in ihrem Geltungsbereich ausgeweitet und in ihren Vorschriften präzisiert. Seit theodosianischer Zeit widerriefen die Kaiser nach und nach alle Konstitutionen, die einzelne heidnische oder häretische Gruppierungen begünstigt hatten - das speciale rescriptum ${ }^{74}$ das oraculum, ${ }^{75}$ die pragmatica sanctio, ${ }^{76}$ das privilegium, ${ }^{77}$ das speciale beneficium ${ }^{78}$-, oder erklärten sie für ungültig, was nicht ausschloss, dass zur Strafverschärfung ein speciale praescriptum erlassen werden konnte. ${ }^{79}$ Theodosius I. und seine Nachfolger promulgierten stattdessen leges generales, ${ }^{80}$ die vor allem daran zu erkennen sind, dass sie dem Anspruch nach eben keine beneficia oder privilegia mehr, sondern über ihren konkreten Anlass hinaus gültige Bestimmungen sein sollten. ${ }^{81}$ Für den Gouverneur bedeutete dies, dass bei den Strafprozessen in Religionsangelegenheiten nicht länger einander widersprechende Konstitutionen, oft zweifelhafter Herkunft, zuweilen auch gefälscht, beigebracht werden konnten. Wer dies dennoch tat, machte sich nun der Fälschung schuldig (crimen falsi). ${ }^{82}$ Ein privilegium, wie es Praetextatus erwirkt hatte, um die Mysterienfeiern in Athen abhalten zu können, war seit Theodosius I. für Heiden und Häretiker kaum mehr zu erlangen ${ }^{83}$ wohl aber für orthodoxe Bi-

72 Zwei Konstitutionen des Jahres 423 sprechen von pagani qui supersunt (Cod. Theod. 16,10,2223), was darauf hindeutet, dass die meisten Heiden ihren Kultus zumindest nicht mehr offen pflegten.

73 Cod. Theod. $16,10,24,1=$ Cod. Iust. 1,11,6.

74 Cod. Theod. 16,5,6 pr.(a. 381); vgl. Cod. Theod. 16,5,5 (a. 379): antiquato rescripto.

75 Cod. Theod. 16,5,51 (a. 410).

76 Cod. Theod. $16,5,25$ pr. (a. 395); $16,5,52$ (a. 412).

77 Cod. Theod. 16,5,17 (a. 389).

78 Cod. Theod. 16,5,58,4 (a. 414).

79 Cod. Theod. 16,5,35 (a. 399) für die Manichäer.

80 Dazu MatTHEws 1993, 26-29; HonORÉ 1998, 128-129; MATTHEWs 2000, 65-70: Als generales leges können die kaiserlichen orationes im Senat gelten; epistulae und decreta an Amtsträger, die als edictum publiziert wurden; Einzelentscheidungen, die über den Fall hinaus gelten sollten, und Entscheidungen, die sich selbst als lex generalis bezeichnen. Die Konzeptionalisierung der lex generalis gegenüber anderen Formen (eingeschränkterer) kaiserlicher Rechtsetzung ist allerdings erst ein Werk des 5. Jahrhunderts (Cod. Iust. 1,14,2-3; a. 426; Cod. Theod. 1,1,5; a. 429), als die Kodifizierung der Rechtstraditionen unter Theodosius II. begann.

81 Als im Jahr 444 ein Kuriale aus Emesa in der Provinz Phoenicia Libanensis sich mit einem gefälschten Kodizill einen höheren Rang als der consularis Syriae anmaßte und den Statthalter beim Tribunal in die zweite Reihe verwies, verfügte Theodosius in diesem speziellen Fall die Aberkennung des angemaßten honor, fügte aber ein allgemeines Gesetz hinzu: verum quia principale remedium generale esse oportet, ... (Nov. Theod. 15,2,2).

82 Cod. Theod. 16,5,16 (a. 388) in Bezug auf die Arianer.

83 Eine Ausnahme bildet die Verfügung, den Haupttempel von Edessa geöffnet zu halten (Cod. Theod. 16,10,18; a. 382 an den dux Osrhoenis). 
schöfe wie Marcellus von Apameia oder Porphyrius von Gaza, die auf ihre Intervention am Kaiserhof hin Reskripte erhielten, welche die Zerstörung von Tempeln anordneten. ${ }^{84}$

Die Präzisierung der Vorschriften diente dazu, die Ambivalenz früherer Gesetze zu vermeiden und deren Interpretationsspielraum aufzuheben. ${ }^{85}$ Theodosius präzisierte mit seiner Gesetzgebung in den Jahren 391/92 die inkriminierten Kultpraktiken und die Strafen, und er präzisierte auch den Charakter des Deliktes. ${ }^{86}$ Was dieses angeht, unterschied Theodosius nun zwischen crimen sacrilegii und publicum crimen. ${ }^{87}$ Waren bislang bestimmte pagane Riten - sofern überhaupt als crimina sacrilegii geahndet worden, ${ }^{88}$ so wurden jetzt das Tieropfer und alle Formen der Divination ${ }^{89}$ analog zum Majestätsverbrechen als publicum crimen verfolgt. Erst im Jahr 472 erklärte dann Kaiser Leo I. die gesamte pagane Kultpraxis zum publicum crimen. ${ }^{90}$

Was die Strafen betrifft, so ist keine Tendenz zu einer kontinuierlichen Verschärfung erkennbar. Vielmehr verfügte Constantius II. im Jahr 354 nicht nur die Schließung aller Tempel, sondern untersagte bei Todesstrafe das Tieropfer und wiederholte dies zwei Jahre später, wobei er die Verehrung von Götterbildern mit

84 Apameia (a. 388): Thdt. h.e. 5,21,1 mit FOWDEN 1978, 64-65; Gaza (a. 398 und 402): Marc.

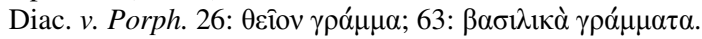

85 Etwa Cod. Theod. 16,5,61 (a. 423): omnis dubiae interpretationis ambages hac sententia revocantes publicari praecipimus.

86 Cod. Theod. 16,10,10-12.

87 Cod. Theod. 16,10,12: utpote violatae religionis reus einerseits, ad exemplum maiestatis reus andererseits. Die Begründung dafür, dass die manichäische Kultpraxis insgesamt als publicum crimen gewertet wird, lautet im Jahr 407 (Cod. Theod. 16,5,40,1): ac primum volumus esse publicum crimen, quia quod in religionem divinam conmittitur, in omnium infertur iniuriam.

88 Cod. Theod. 16,2 [de episcopis, ecclesiis et clericis], $25=$ Cod. Iust. 9,29 [de crimine sacrilegii], 1 (a. 380): qui divinae legis sanctitatem aut nesciendo confundunt aut neglendo violant et offendunt, sacrilegium committunt. Der Ausdruck divina lex ist ambivalent: er kann sowohl auf göttliches wie kaiserliches Gesetz bezogen werden (vgl. HUNT 1993, 147-148). Unter der Monarchie ist ein sacrilegium nicht mehr bloß ein Verstoß gegen Religionsgesetze, sondern zugleich auch gegen die kaiserliche maiestas; dies gilt a fortiori von den christlichen Kaisern. So untersagten z. B. leges et divinae et imperiales sämtliche Häresien (Cod. Theod. 16,5,5; a. 379). Auch würden die Manichäer ein sacrilegium begehen, da sie die Verknüpfung des ordo caelestium statutorum mit der observantia sacratae constitutionis ignorierten (Cod. Theod. $16,5,7,1$; a. 381).

89 Die Divination wurde der Magie gleichgestellt (Cod. Theod. 16,10,12,1): Wie die ars mathematica mache es sich die divinatio zur Aufgabe, naturae leges rescindere, inlicita perscrutari, occulta recludere, interdicta temptare, finem quaerere, spem polliceri, futura cognoscere, veritatem explorare. $\mathrm{Zu}$ den Implikationen dieser Gleichsetzung FÖGEN 1997, bes. 48-53; vgl. TROMBLEY 1993, I 59-72.

90 Cod. Iust. 1,11,8 pr. (a. 472): nemo ea, quae saepius paganae superstitionis hominibus interdicta sunt, audeat pertemptare, sciens, quod crimen publicum committit qui haec ausus fuerit perpetrare. Zum strafrechtlichen Umgang mit Häretikern und Heiden allgemein MOMMSEN 1899, $601-610$. 
einbezog. ${ }^{91}$ Gegenüber dieser in jeder Hinsicht pauschalen Sanktion milderten die nächsten überlieferten Gesetze die Strafen und differenzierten sie nach Stand und Delikt. Das Instrumentarium reichte von der Geldstrafe (multa) ${ }^{92}$ der Körperstrafe (verbera) und der Konfiskation (bonorum proscriptio) über das Exil und die Deportation bis zum Ausschluss aus dem kaiserlichen Dienst (militia). Erst im Jahr 435 wurde wieder pauschal die Zerstörung der noch verbliebenen Tempel und die Todesstrafe für alle Personen angeordnet, die heidnischer Kultpraktiken überführt waren. ${ }^{93}$ Aufschlussreich ist aber, dass gegen die Heiden - anders als gegen die Häretiker - nicht die Strafe der infamia und die damit verbundenen zivilrechtlichen Diskriminierungen (Testamentsverbot, Testierverbot; Kontrak-

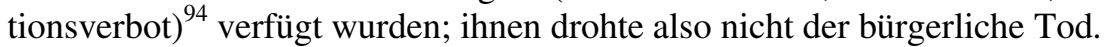

Während die Kaiser Constans und Constantius II. beim Verbot heidnischer Kultpraktiken zwischen dem Vollzug von sacrificia, der Verehrung von simulacra, dem Besuch von templa und der Praxis der superstitio, das heißt der Wahrsagung, differenzierten, wurde Theodosius I. konkreter: Er untersagte nicht nur die öffentlichen ,blutigen' Opfer, sondern auch den häuslichen Kult des Genius, der Laren und der Penaten; nicht nur alle Formen der Wahrsagung, sondern auch die ländlichen Riten der Darbringung von Feldfrüchten, der Bekränzung von Bäumen, des Weihrauchopfers und anderes mehr. ${ }^{95}$ Durch die genaue Auflistung aller erdenklichen Riten und Zeremonien wurde nun jeder Interpretationsspielraum darüber beseitigt, was noch erlaubt sei und was nicht mehr. So war das Verbot der superstitio in der ersten Hälfte des 4. Jahrhunderts keineswegs eindeutig gewesen, da der Begriff sowohl magische Praktiken als auch den heidnischen Opferkult bezeichnen konnte. ${ }^{96}$ Der Begriff des sacrificium wiederum meinte sowohl das ,blutige' wie das frugale Opfer. Und der Besuch von Tempeln mochte dem Opfer dienen oder bloß dem Gebet zu einer Gottheit, ganz zu schweigen von den profanen Aktivitäten, die im Tempelbezirk stattfanden.

91 Cod. Theod. 16,10, 4 (= Cod. Iust. 1,11,1) und 10,6: poena capitis subiugari praecipimus eos, quos operam sacrificiis dare vel colere simulacra constiterit. Ein Reflex des Gesetzes von 354 findet sich bei Soz. h.e. 4,10,7: Den Magistraten von Alexandreia wird ein kaiserliches Schrei-

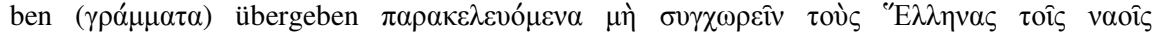

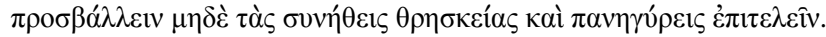

92 Die Mult konnte nach fünfmaliger Verurteilung freilich in eine schärfere Leib- oder Vermögensstrafe umgewandelt werden ( $\mathrm{Cod}$. Theod. 16,5,54,3; a. 414). Die Geldstrafen sind rangmäßig differenziert (Cod. Theod. 16,5, 52; a. 412; 16,5,54; a. 414; 16,10,10; a. 391; Const. Sirmond. 12).

93 Cod. Theod. 16,10,25; vgl. Nov. Theod. 3,8 (a. 438).

94 Zum Beispiel Cod. Theod. 16,5,40,3-4 (a. 407 mit Bezug auf die Manichäer und Priscillianisten): ipsos quoque volumus amoveri ab omni liberalitate et successione quolibet titulo veniente. praeterea non donandi, non emendi, non vendendi, non postremo contrahendi cuique convicto relinquimus facultatem.

95 Cod. Theod. 16,10,12.

96 SALZMAN 1987, 177-179.

97 Vgl. MaCMuLLEN 1984, 97. 
Die Unklarheit darüber, was die kaiserlichen Gesetze nun verboten und was nicht, geht aus der Rede des Libanios gegen die Zerstörung der Tempel, die er wohl in den Jahren zwischen 385 und 387 verfasst hatte, deutlich hervor: ${ }^{98}$ Libanios weiß nichts von einem Opferverbot des Constantin, und dasjenige des Constantius wurde durch Julians Maßnahmen aufgehoben ${ }^{99}$ und danach nur für das Tieropfer wieder erneuert. ${ }^{100}$ Ein derartiges Gesetz ist allerdings im Codex Theodosianus nicht überliefert, denn Gratian, Valentinian II. und Theodosius I. verboten in den Jahren 381 und 385 nur solche sacrificia, die mit divinatorischen Riten verbunden waren. ${ }^{101}$ Nach den im Codex Theodosianus erhaltenen Gesetzen zu urteilen, wurde auch bis zum Jahr 391 keine Schließung der unter Julian wieder geöffneten Tempel verfügt. Im Gegenteil: Die einzige diesbezügliche Konstitution ordnete die Öffnung des Haupttempels im überwiegend christlichen Edessa an, allerdings unter Verzicht auf prohibitorum usus sacrificiorum. ${ }^{102}$ Während also unter Constantius allen Heiden noch bewusst war, dass ,blutige ' Opfer und der Zugang zu den Heiligtümern verboten waren und sanktioniert wurden, ${ }^{103}$ scheint in der Zeit nach Julians Herrschaft bis zu den einschneidenden Gesetzen des Theodosius im Jahr 391/92 die kaiserliche Auffassung zum paganen Kultus bis auf ein Verbot ,blutiger' Opfer unklar gewesen zu sein. ${ }^{104}$ So konnte Libanios in seiner Rede für Theodosius behaupten, dass dieser selbst weder die Opfer verboten, noch den Zugang zu den Tempeln verwehrt hätte. ${ }^{105}$

Die Durchsetzung der kaiserlichen Religionspolitik gegenüber den paganen Kulten war bis gegen Ende des 4. Jahrhunderts also bereits unter einem rechtlichen Aspekt selbst für gutwillige Gouverneure mit großen Unsicherheiten be-

98 Lib. or. 30 [Pro templis], 6-9. Die Publikation erfolgte wohl nicht vor dem Tod des praefectus praetorio Orientis (384-388) Maternus Cynegius im Jahr 388 (vgl. PLRE I 235-236, Cynegius 3). Zu dieser Datierung WIEMER 1995, 123-129.

99 Ebenso Amm. 22,5,1: planis absolutisque decretis aperire templa arisque hostias admovere et restituere deorum statuit cultum.

100 Jedenfalls ist ein solches Gesetz nicht im Codex Theodosianus erhalten. Lib. or. 30,7 spricht

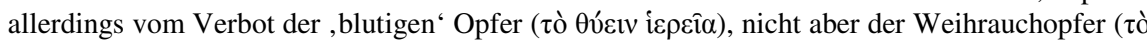
$\left.\lambda_{1} \beta \alpha v \omega \tau o ́ v\right)$ durch Valentinian I. und Valens.

101 Cod. Theod. 16,10,7 und 9, letzteres an den praefectus praetorio Orientis Maternus Cynegius.

102 Cod. Theod. 16,10,8 (a. 382). Diese Bestimmung könnte, wenn sie hier nicht ad hoc getroffen wurde, auf ein nicht überliefertes Gesetz gegen das Tieropfer zurückgehen.

103 Mit dem Verbot der Opfer kam der ganze städtische Kult zum Erliegen (wenn er nicht schon zuvor aufgegeben worden war); vgl. nur Lib. or. 18 [Epitaphios], 23 mit rhetorischer Übertrei-

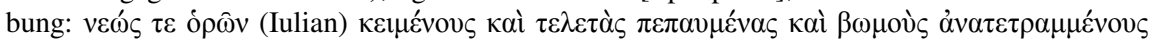

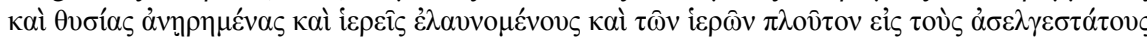
$\mu \varepsilon \mu \varepsilon \rho ı$ เ̇́vov. Vgl. Soz. h.e. III 17,2-3; Liban. or. 1,119; or. 14,41 (a. 362), or. 18,114. Die Bedeutung der Opfer für die Aufrechterhaltung des städtischen Kults unterstreicht HARL 1990, $8-13$.

104 Glaubt man Theodoret (Thdt. h.e. 4,24,2), so gewährte Kaiser Valens in seiner Residenz Antio-

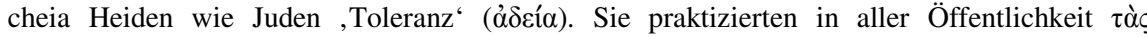

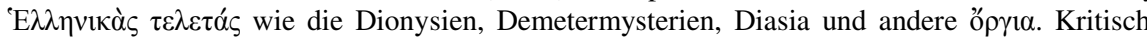
gegenüber diesem Bericht HAHN 2004, 186, Anm. 275, freilich ohne nähere Begründung.

105 Lib. or. 30,8. Auch FOWDEN 1978, 62 spricht für diese Zeit von de facto-Toleranz. 
lastet: Die Gewährung von beneficia oder privilegia und die dadurch hervorgerufene Entwertung bereits erlassener Gesetze einerseits sowie die unklare Rechtslage bezüglich paganer Kultpraktiken in der Zeit nach Julian andererseits müssen sich auf die Prozesse vor dem Tribunal des Statthalters ausgewirkt haben. Hier haben erst die gesetzgeberischen Maßnahmen des Theodosius in den 390er Jahren rechtliche Klarheit geschaffen.

Der statthalterliche Strafprozess ${ }^{106}$ hatte sich gegenüber der Principatszeit kaum verändert. Die Prozesse wegen sacrilegium oder publicum crimen, in denen es unter anderem um Folterung, Verbannung, Personenstand und Hinrichtung ging, ${ }^{107}$ hatte der Gouverneur selbst mit seinem assessor und dem Rat seines consilium zu führen und konnte sie nicht an pedanei iudices delegieren. ${ }^{108}$ Das Statthaltergericht war freilich nicht nur mit den strafrechtlichen Aspekten der Prozesse gegen die Heiden befasst. Die Konfiskation und Neuverteilung von Tempelland und Tempeleinkünften; ${ }^{109}$ die Aufhebung der Privilegien heidnischer Priester; ${ }^{110}$ die Einziehung der Güter von Heiden, die den Kult auf ihren Ländereien zugelassen oder selbst praktiziert hatten; ${ }^{111}$ der mehrfach von den Kaisern verfügte Schutz der Tempel als öffentliche Gebäude und Schmuck der Städte (publicorum operum ornamenta); ${ }^{12}$ die Aufrechterhaltung des städtischen Festwesens ohne

106 Dazu allgemein Mommsen 1899, 229-250 und für die Spätantike SANTALuCIA 1998, 269-276 und 280-286; zur cognitio extra ordinem der Statthalter 227-233.

107 Nachrichten zum statthalterlichen Strafprozess stammen besonders aus der Zeit Julians (als Zeit

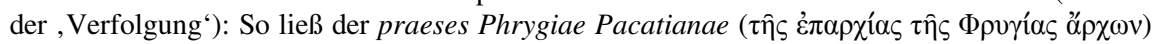
Amachius Christen, die nachts Kultbilder des wiedereröffneten Tempels in Meiros zerstört hatten, foltern und hinrichten, nachdem sie sich geweigert hatten, sich vor dem Tribunal zu rechtfertigen ( $\dot{\alpha} \pi \mathrm{o} \lambda \mathrm{\sigma} \varepsilon \varepsilon \hat{\imath} \sigma \theta \alpha$; Socr. h.e. 3,15; Soz. h.e. 5,11,1; PLRE I 50). Einen Strafprozess gegen Heiden im Jahr 482/83 schildert anschaulich Zach. v. Sev. 23-27; vgl. TROMBLEY 1994, II 1015. Zum analogen kaiserzeitlichen Tribunal gegen Christen MEYER-ZWIFFELHOFFER 2002, 143-171.

108 Cod. Theod. 1,16 [de officio rectoris provinciae], $8=$ Cod. Iust. 3,3 [de pedaneis iudicibus], 5 (a. 362): ideoque pedaneos iudices, quod est qui negotia humiliora disceptent, constituendi damus praesidibus potestatem; dazu MommSen 1899, 248-250; SANTAluCia 1998, 272. Auch die städtischen Behörden durften causae criminales nur in geringfügigen Fällen (res parvae ac minimae) entscheiden (Cod. Theod. 2,1 [de iurisdictione], 8,1; a 395). Selbst die Delegation der Rechtsprechung an die Legaten des Prokonsuls unterlag strikten Restriktionen (Cod. Theod. 1,12 [de officio proconsulis et legati], 8; a. 400).

109 Const. Sirmond. 12 = Cod. Theod. 16,10,19,2 (a. 407): ad usum publicum; 10,19 pr.: an die annona militaris; 10,20,1 (a. 415): an die res privata. - Julian hatte mit einem Gesetz vom 3. Februar 362 die Rückgabe eingezogener Tempelgüter oder deren Kompensation verfügt (Hist. aceph. 3,1 [ed. Martin]; vgl. Lib. or. 18,126 und 129; Soz. h.e. 5,3,1-2; 4; 5,1-2), was von Valentinian I. und Valens zwei Jahre später wieder zugunsten der res privata rückgängig gemacht wurde: Cod. Theod. 10,1 [de iure fisci], 8 (a. 364) und 5,13 [de fundis rei privatae et saltibus divinae domus], 3 (a. 364).

110 Cod. Theod. $16,10,14$ (a. 396).

111 Cod. Theod. 16,10,12,3 (a. 392); 10,20,1 (a. 408).

112 Cod. Theod. 16,10,15 = Cod. Iust. 1,11,3 (a. 399): sicut sacrificia prohibemus, ita volumus publicorum operum ornamenta servari; 16,10,18 (a. 399): aedes inlicitis rebus vacuas nostrarum beneficio sanctionum ne quis conetur evertere (vgl. 16,10,3; a. 342). Obwohl Theodosius 
Opferriten; ${ }^{113}$ die Neuordnung des städtischen Kalenders im christlichen Sinne waren zivilrechtliche Vorgänge, die teils aus den Strafprozessen resultierten, teils unabhängig davon Aspekte der statthalterlichen Aufsicht über die städtische und provinziale Ordnung betrafen. Was das Festwesen anbelangt, das den städtischen Lebensrhythmus seit jeher grundlegend geprägt hatte, taten sich einzelne Gouverneure mit den kaiserlichen Konstitutionen schwer und haben diese offenbar nur zögernd oder gar nicht umgesetzt: So musste noch im Jahr 395 der corrector Paphlagoniae Heraclianus in einem vom Kaiser direkt an ihn adressierten Schreiben ermahnt werden, dass die heidnischen Feste keine feriae mehr waren. ${ }^{114} \mathrm{Zu}$ gleich aber ließ Honorius im Jahr 399, als er die Tempel in Africa zu schließen befahl, ${ }^{115}$ die Festkultur als öffentliche Unterhaltung (communis laetitia) unangetastet. ${ }^{116}$ Auch hier bestand die Unsicherheit, ob pagane Feste ohne ihre inkriminierten Kultpraktiken bestehen bleiben konnten und ob Christen daran teilnehmen durften. ${ }^{117}$

3. Einige kaiserliche Konstitutionen bedrohten die römischen Amtsträger und provinzialen Machthaber, die die Anwendung der Religionsgesetze behinderten, mit Geld-, Leib- und Vermögensstrafen. Sie warnten die iudices, officiales und curiales davor, untätig zu bleiben (desidia), sich gleichgültig den Verbrechen (neglegentia, incuria) oder voller Geringschätzung den Gesetzen gegenüber (contemptus) zu verhalten, im geheimen Einverständnis mit den Delinquenten zu stehen (coniventia) oder aus Gefälligkeit ihnen gegenüber zu handeln (gratia) und dann so zu tun, als wüssten sie nichts von den Vorfällen (dissimulatio). ${ }^{118}$ Kon-

\footnotetext{
II. im Jahr 435 die Zerstörung aller Tempel angeordnet hatte (Cod. Theod. 16,10,25), verschärfte im Jahr 458 Maiorian mit einer generalis lex den Schutz der Tempelgebäude in Rom und den Provinzen, nun vor christlichen Amtsträgern (Nov. Maior 3 [de aedificiis publicis], 1): idcirco generali lege sancimus cuncta aedificia quaeve in templis aliisque monumentis a veteribus condita propter usum vel amoenitatem publicam subrexereunt, ita a nullo destrui atque contingi, ut iudex, qui hoc fieri statuerit, quinquaginta librarum auri inlatione feriatur. Dass die Zerstörung heidnischer Tempel in den Städten dennoch Praxis war, ergibt sich nicht nur aus den Vorfällen um das Serapeion oder das Marneion, sondern auch aus der beiläufigen Anweisung an einen comes Orientis, für den Bau von Straßen, Brücken und Aquädukten das Material ex demolitione templorum zu benutzen (Cod. Theod. 15,1 [de operibus publicis], 36 (a. 397).
}

113 Cod. Theod. 16,10,3 (a. 346); 16,10,17 (a. 399).

114 Cod. Theod. 2,8 [de feriis], 22: sollemnes paganorum superstitionis dies inter feriatos non haberi olim lege reminiscimur imperasse (PLRE I 417, Heraclianus 4; vgl. Cod. Theod. 2,8,19; a. 389). Außer den christlichen Festen und dem Sonntag gehörten der Neujahrstag, die dies imperii und dies natales des Kaiserhauses und die Gründungstage von Rom und Constantinopel zu den feriae.

115 Aug. civ. 16,II 54; Quodvultd. I prom. 3,38,41.

116 Cod. Theod. 16,10,17 = Cod. Iust. 1,11,4 (a. 399 an den proconsul Africae): ut profanos ritus iam salubri lege submovimus, ita festos conventus civium et communem omnium laetitiam non patimur submoveri.

117 Dazu MARKUS 1990, 107-123.

118 Cod. Theod. 16,10,4 (a. 354); 16,5,4 (a. 376); 16,5,12 (a. 383); 16,10,12,4 (a. 391); 16,10,13,1 (a. 395); 16,7 [de apostatis], 4,4 (a. 405); Const. Sirmond. 12 (a. 407); 14 (409); Cod. Theod. 16,5,65,5 (a. 428); Cod. Iust. 1,11,7,3 (a. 451). 
kret hieß dies, dass die städtischen Behörden sowie die stationarii dem Statthalter nicht über die vorgefallenen Delikte Bericht erstatteten (in notitiam deferre), weil sie Sympathisanten waren ${ }^{119}$ beziehungsweise bestochen wurden; ${ }^{120}$ oder sie wagten es nicht, ihn darüber zu informieren, da sie um sein Desinteresse an einer Verfolgung wussten. Doch selbst wenn die Delinquenten rechtmäßig vor dem Tribunal angeklagt und verurteilt wurden, gab es Gouverneure, die anschließend die Bestrafung unterließen (dissimulaverit vindicare) ${ }^{121}$ oder mildere Strafen verhängten, als sie für das Delikt vorgesehen waren. Manche Statthalter wie der Prokonsul Porphyrius unterrichteten nicht einmal den Kaiser über die religiösen Unruhen in ihrer Provinz, obwohl dies ihre Pflicht war. ${ }^{122}$

Solche Verhaltensweisen setzten ein Einverständnis zwischen dem Gouverneur und seinem officium voraus. ${ }^{123}$ Dieser besaß über seine officiales einerseits

119 Cod. Theod. 16,4 [de his, qui super religione contendunt], 4 (a. 404): cuncta officia moneantur a tumultuosis se conventiculis abstinere, et qui sacrilego animo auctoritatem nostri numinis ausi fuerint expugnare, privati cingulo bonorum proscribtione multentur.

120 Vgl. die Bestimmungen gegen die rapaces officialium manus schon unter Constantin: Cod. Theod. 1,16,7 (a. 331), wobei es nicht um ein generelles Verbot der sportulae ging, sondern um deren Höchstgrenze. Zur ,Korruption“ provinzialer Amtsträger vgl. VEYNE 1981 (der sportulae und suffragia als wesentlich für das Funktionieren vormoderner, Verwaltungen' betrachtet); CORCORAN 1996, 239-244; HARRIES 1999, 153-171. Von ,Korruption“ könnte nur dort gesprochen werden, wo die Höchstgrenzen für sportulae (vgl. den ordo commodorum aus Timgad, Chastagnol 1978, 76, Z. 12-51) willkürlich überschritten wurden. - Bestechen ließen sich dagegen kaiserliche Sondergesandte wie der subadiuva des magister officiorum, der im Jahr 398 die Götterbilder in Gaza zerstören und die Tempel schließen sollte. Er führte seinen Auftrag aus,

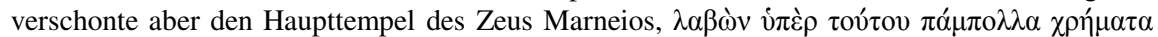
(Marc. Diac. v. Porph. 27).

121 Cod. Iust. 1,11,7,3 (a. 451): post accusationem legitimam et post crimen in cognitione convictum.

122 Const. Sirmond. 14: debita cura referendi in nostram notitiam.

$123 \mathrm{Zu}$ den officia der Statthalter, die von einigen höheren Chargen (promoti) und der Masse der exceptores gebildet und deren Mitglieder als cohortalini (cohortales) bezeichnet wurden (vgl. Cod. Theod. 8,4 [de cohortalibus], 12; a. 372), siehe JONES 1964, 593-596; 744; PALME 1999, 100-121. BAGNALL 1993, 65-67, geht von durchschnittlich 100 officiales in den Büros der ordinarii iudices aus. Der proconsul Africae und seine zwei Legaten durften 400 officiales intra numerum beschäftigen (Cod. Theod. 1,12 [de officio proconsulis et legati], 6; a. 398). Der princeps officii war in den Provinzen Asia und Africa Proconsularis sowie anderen wichtigen Provinzen ein ehemaliger agens in rebus, der den Statthalter auch zu kontrollieren hatte (Cod. Iust. 4,28 [de principibus agentum in rebus], 6; vgl. JONES 1964, 128-129) und dem magister officiorum unterstand (vgl. PALME 1999, 108). Die Statthalter besaßen auch Disziplinargewalt ihren cohortales gegenüber (Cod. Iust. 1,40 [de officio rectoris provinciae], 12; a. 412). Zur Struktur der officia beispielhaft Not. Dig. or. XX-XXI und occ. XVIII (proconsul), or. XLIII und occ. XLIV (consularis), or. XLIII und occ. XLV (praeses), occ. XLIV (corrector). - Alle Diskriminierungen der Heiden, Häretiker und Juden rührten jedoch nicht an dem Grundsatz, dass die korporativen Zwangsverpflichtungen, die mit der Erblichkeit des Kohortalinenstandes verbunden waren (Cod. Theod. 6,35 [de privilegiis eorum, qui in sacro palatio militant], 14,2; a. 423; JONES 1964, 594; 743), erfüllt werden mussten: Jene konnten sich weder dem ordo decurionum noch der militia cohortalina entziehen: si quos vero ex his (haereticis) curialis origo vel ordinum nexus aut cohortalinae militiae inligat obsequiis et functionibus, his adstringi praeci- 
Disziplinargewalt, andererseits informelle Macht, da er über Beförderungen und Freistellungen entschied. Er konnte sie an übermäßigen sportulae verdienen lassen und darüber hinwegsehen, wenn seine officiales gegen Vorschriften verstießen. Als Vermittler von Karrierechancen war dem Statthalter eine Machtfülle gegeben, welche die kaiserlichen Konstitutionen vergeblich einzudämmen versuchten. Deshalb wohl waren die Geldstrafen für den Statthalter genauso hoch bemessen wie diejenigen für sein officium. ${ }^{124}$. Die officiales blieben nur dann straffrei, wenn sie ihren Gouverneur nachweislich auf die vorgeschriebenen Verfahrensweisen hinwiesen hatten (suggestio). ${ }^{125}$ Damit wurde das officium gezielt dazu angehalten, seinen Statthalter zu kontrollieren und sich gegebenenfalls gegen ihn zu stellen. ${ }^{126}$

Die ständige Wiederholung von Gesetzen gegen Heiden und Häretiker, die Strafdrohungen gegen untätige oder unwillige Amtsträger und die zunehmende Verschärfung des Tons in den Konstitutionen werfen die Frage auf, ob darin ein Zeugnis für die Unwirksamkeit der kaiserlichen Gesetzgebung gesehen werden muss oder ob die Gesetzgebung auch anderen Zwecken diente als der uns heute geläufigen unverzüglichen Exekution. Was die Unwirksamkeit betrifft, so ist nach dem bisher Dargelegten deutlich geworden, dass die Erzwingung von gesetzlichen Maßnahmen prinzipiell von der lokalen Kräftekonstellation abhängig war, in welcher der Gouverneur auf die Mithilfe der örtlichen Machthaber und seines gleichfalls lokal rekrutierten officium angewiesen war. ${ }^{127}$ Ein weiteres strukturelles Problem ergab sich aus dem Widerspruch zwischen den konkreten Konflikten, welche die Kaiser mit ihren Reskripten und Dekreten beizulegen trachteten, und ihrem Versuch, mittels leges generales eine allgemeine Religionspolitik dem

pimus, ne sub colore damnatae religionis eliciant vacationis cupitae sibi suffragia (Cod. Theod. 16,5,48; a. 408; vgl. 16,5,65,3; a. 423 mit Bezug auf die Manichäer). Der Grund dafür lag wohl darin, dass die militia cohortalis wie der ordo decurionum zur untersten Gruppe der honestiores gehörte (Cod. Theod. 6,35,14,1: inferioris sortis homines und: condicionis macula).

124 In der Regel im Westen 20 Pfund Gold (1440 Solidi), im reicheren Osten 30 Pfund Gold (2160 Solidi). Im Jahr 395 wurde die Multstrafe im Osten ergänzt für den Statthalter um die Strafsumme, welche die Verurteilten zu zahlen hatten. Seine officiales wurden mit dem capitale supplicium bedroht (Cod. Theod. 16,10,13,1). Im Westen verlor der Stathalter seit 409 auch seine dignitas (Const. Sirmond. 14). Im Jahr 451 lag die Strafe für den Statthalter und sein officium bei immerhin je 50 Pfund Gold (3600 Solidi; Cod. Iust. 1,11,7). - Analog zu der Bestrafung entsprach das Gehalt des Statthalters in etwa dem Gehalt seiner sämtlichen officiales; zum Gehalt PALME 1999, 112-113.

125 Const. Sirmond. 14.

126 Cod. Theod. 16,10,11 (a. 391): officium vero eius (iudicis), nisi conlatis viribus obviarit, parem summam aerario nostro inferre cogatur. Zur Kollektivhaftung des officium ROSEN 1990.

127 Wie das album decurionum aus Timgad zeigt, diente ein Viertel der Dekurionen (70 Personen) in den officia des consularis Numidiae (37), des vicarius Africae (5), des praefectus annonae (23) und des rationalis (5). Dies sind die im ordo salutationis genannten officiales ex ordine ( $\mathrm{Z}$. 12); vgl. Chastagnol 1978, 94-99. Hier wird die enge Verknüpfung von Provinzial- und Kommunalverwaltung deutlich. (PALME 1999, 119). Doch konnten sie auf diese Weise nicht den kurialen munera entkommen: man rief sie entweder zurück oder bestimmte, dass die munera geleistet werden mussten (JONES 1964, 744). 
jeweiligen Reichsteil zu verordnen. Ob eine religiöse Gruppierung Privilegien erwirken konnte, hing immer auch davon ab, wie sehr der Kaiser in diesem Moment auf sie angewiesen war. Bei der Anwendung der Religionsgesetze kam es dann zu weiteren konfliktträchtigen Interessenslagen, etwa ob die Aufrechterhaltung der öffentlichen Ordnung oder die religiöse Disziplin Vorrang genießen ${ }^{128}$ bzw. ob die Christianisierung ohne Rücksicht auf die steuerzahlenden Gemeinden durchgesetzt werden sollte. ${ }^{129}$

Was den Charakter der Gesetzgebung angeht, so hat bereits Paul Veyne hervorgehoben, dass die rhetorisch ausgefeilten Konstitutionen nicht nur Vorschriften zur Disziplinierung einzelner Bevölkerungsgruppen enthalten, sondern auch Proklamationen eines moralischen Ideals, für das der Herrscher einzustehen vorgab. ${ }^{130}$ Er bekundete auf diese Weise reichsweit, dass er seinen Aufgaben als Herrscher christlichen bzw. nizänischen Bekenntnisses mit Eifer nachkam und die Feinde des wahren Glaubens unerbittlich bekämpfte. Das sanktionsbewehrte Gebot der Rechtgläubigkeit, die Theodosius I. zur allgemeinen Richtlinie erhoben hatte und die auch der Legitimierung seiner Religionsgesetze diente, schuf - wie Peter Brown betont hat - eine Atmosphäre der Einschüchterung und der Diskriminierung. ${ }^{131}$ Darin aber lag laut dem Rechtsgelehrten und Kirchenhistoriker Sozomenos der eigentliche Zweck der theodosianischen Religionsgesetze: ${ }^{132}$

In den Gesetzen schrieb er strenge Strafen fest, wandte sie aber nicht an, denn er wollte die Untertanen nicht bestrafen, sondern einschüchtern, damit sie über das Göttliche gleicher Meinung wie er würden. Ja er lobte auch diejenigen, die freiwillig (zur nizänischen Kirche) konvertierten.

Sozomenos bestätigt den Befund unserer Quellen, in denen von praktizierter Bestrafung opfernder Heiden und untätiger Amtsträger vor dem 4. Jahrhundert keine Rede ist. Außerdem verfügten viele Gesetze gegen Heiden und Häretiker wie die Konstitution des Honorius aus dem Jahr 407 Straffreiheit für alle, die sich der

128 In der Auseinandersetzung um die Zerstörung der Synagoge in Kallinikon im Jahr 388 schrieb Ambrosius an Theodosius (ep. 40,11): sed disciplinae te ratio, imperator, movet. quid igitur est amplius? disciplinae species, an causa religionis? $\mathrm{Zu}$ dieser Episode und ihrer Interpretation siehe den Beitrag von GOTTER in diesem Band.

$129 \mathrm{Vgl}$. oben Anm. 52.

130 VEYNE 1981, 341 mit Anm. 26.

131 BROWN 1963, 285-294.

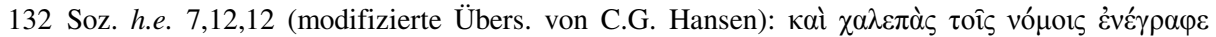

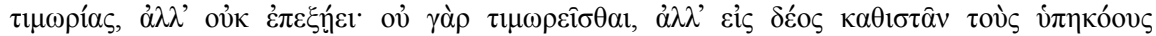

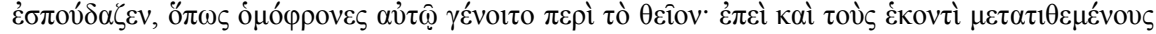

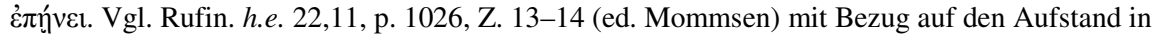
Alexandreia anlässlich der Zerstörung des Serapeion: ille (Theodosius I.), qui ingenita mentis clementia errantes mallet emendare quam perdere. In diesem Fall hat wahrscheinlich der pagane Prätorianerpräfekt Tatianus (388-392) die kaiserliche clementia erwirkt. 
nizänischen Kirche zuwandten. Denn es ging dem Kaiser darum, seine Untertanen $\mathrm{zu}$ „verbessern“ (emendare bzw. corrigere), nicht sie zu vernichten. ${ }^{133}$

Die Atmosphäre von Angst und Diskriminierung wirkte latent, doch wurde sie in konfliktgeladenen Situationen virulent. Denn die Gesetze fungierten - um eine Wendung Ramsay MacMullens aufzugreifen - als „licences to take action“. ${ }^{134}$ Es bedurfte demnach bloß interessierter Parteien, um das „Gesetz als Waffe zu gebrauchen“, ${ }^{135}$ und es waren nicht in erster Linie die römischen Amtsträger, die davon Gebrauch machten, am wenigsten aber die Statthalter.

\section{Die Rolle der Statthalter bei gewaltsamen Aktionen gegen den heidnischen Kult}

Die Konstitutionen erwecken den Eindruck, dass die Statthalter die wichtigsten Agenten der provinzialen Christianisierungspolitik gewesen sind oder wenigstens dafür bestraft wurden, wenn sie sich dieser Aufgabe entzogen oder verweigert haben. Zieht man nun die erzählenden Quellen hinzu, die über Christianisierungsbemühungen in den Provinzen Auskunft geben, so ergibt sich ein anderes Bild: Gouverneure spielen dabei keine aktive Rolle, sofern sie überhaupt anwesend sind; statt ihrer agieren Bischöfe und Äbte mit ihrem Gefolge, zum Teil mit Hilfe kaiserlicher Sondergesandter oder militärischer Amtsträger. Dieses Bild ergibt sich zunächst aus der Art der überwiegend christlichen Historiographie: So wie der Codex Theodosianus durch die Auswahl und Anordnung der darin aufgenommenen Konstitutionen eine rückwirkende Interpretation der kaiserlichen Religionspolitik auf die Erfolge der eigenen Gegenwart hin vornahm, so stilisierte und dramatisierte die christliche Historiographie, die nicht von ungefähr zur Zeit Theodosius' II. die Tradition des Eusebius wieder aufnahm, ${ }^{136}$ die Auseinandersetzungen der katholischen Kaiser und Bischöfe mit den heterodoxen und heidnischen Gruppierungen. Und wie der Codex Theodosianus aus imperialer christlicher Perspektive den Untergang des Heidentums konstatierte - pagani qui supersunt $-{ }^{137}$ so proklamierte die christliche Historiographie den Triumph des nizäni-

133 Const. Sirmond. 12: quicumque igitur haereticorum, sive Donatistae sint sive Manichaei vel cuiuscumque alterius pravae opinionis ac sectae profanis ritibus adgregati, catholicam fidem et ritum, quem per omnes homines cupimus observari, simplici confessione susceperint [...] ab omni noxa absolvendos esse censemus.

134 MacMullen 1984, 95; vgl. VeYnE 1981, 348: «Comme souvent à Rome, la règle servait seulement de prétexte ou de cadre à un choix individuel dont on se gardait bien de parler et où les liens de clientèle l'emportaient. »

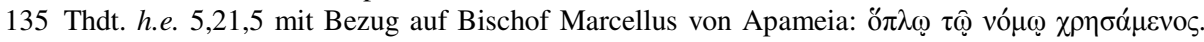
So auch der Titel des Beitrages von HAHN in diesem Band.

136 Die Kirchengeschichten des Rufinus (403), Sokrates (439), Sozomenos (443) und Theodoret (449) stammen wie Theodorets Mönchsgeschichte (440) aus der 1. Hälfte des 5. Jahrhunderts. Zu ihrer Charakteristik unter dem hier behandelten Gesichtspunkt BROWN 1992, 126-134.

137 Cod. Theod. 16,10,22-23 (a. 423); vgl. BROWN 1998, 638-641. 
schen Christentums über seine Gegner. ${ }^{138}$ Für das 5. Jahrhundert bedeutete eine solche Haltung dann, dass eine ,ideology of silence“ die Fortdauer des paganen Kultes in vielen Gegenden des Reiches verdeckte. ${ }^{139}$

Fragt man nun nach der Rolle der Statthalter bei den Auseinandersetzungen mit den paganen Kulten, so muss unterschieden werden zwischen ihrer Funktion als iudices ordinarii, die ihnen auch nicht durch Bischöfe oder militärische Amtsträger streitig gemacht wurde, und der Initiative bei der Durchsetzung religionspolitischer Maßnahmen, das heißt der Ausführung von Gesetzen etwa über Tempelschließungen oder die Zerstörung von Götterbildern sowie dem Aufspüren all derjenigen, die sich der vera religio widersetzten. Im zweiten Fall bedurfte es besonderen Eifers, und diesen zeigten die meisten Statthalter anscheinend nicht.

Dass die Gouverneure nicht aktiv gegenüber Heiden vorgingen (im Gegensatz etwa zum Eifer eines Donatus gegenüber den Donatisten) verwundert zunächst, denn von Julian wird berichtet, dass er seine Statthalter mit der Durchsetzung von Maßnahmen gegen den christlichen Kultus beauftragt hatte, etwa den praeses Cariae mit der Zerstörung von Märtyrergräbern im Tempel von Didyma ${ }^{140}$ oder den praeses Phrygiae Amachius mit der Tempelöffnung in Meiros. ${ }^{141}$ Übergeht also die Überlieferung eine Beteiligung der Gouverneure bei Maßnahmen gegen die Heiden nur deshalb, weil diese ohne großes Aufheben ihre Pflicht getan haben? Dafür könnte sprechen, dass die Quellen in der Regel ja nur über konflikthaltiges und gewaltsames Vorgehen gegen die Heiden bzw. über deren Widerstand berichten. Dass es vielerorts reibungslos zuging, ist also nicht von der Hand zu weisen, jedoch auch nicht zu belegen. Dafür spricht weiter, dass der heidnische Kult in einigen Städten oder Regionen ohnehin bereits seine Anziehungskraft verloren hatte und Tempel sowie Kultbilder verfielen oder das pagane Festwesen verkümmerte. ${ }^{142}$ Hier konnten die kaiserlichen Vorschriften mühelos umgesetzt werden. Auch hatte sich vielerorts der Kultus von den urbanen Zentren mit ihrer traditionellen städtischen Festkultur ab- und ländlichen Kultpraktiken bei Schreinen, Grotten oder heiligen Bäumen zugewandt, die weniger leicht auffielen und kontrolliert werden konnten.

Die Strafandrohungen zeigen allerdings, dass es in anderen Fällen eben nicht reibungslos verlief. Wenn trotzdem nichts von einer Bestrafung unwilliger Gouverneure verlautet, besagt dies nur, dass sie anscheinend nicht in spektakulären Prozessen verurteilt wurden. Wie sich eine solche Haltung allerdings auf ihre weitere Karriere ausgewirkt hat, entzieht sich unserer Kenntnis, doch bot sich hier

138 BROWN 1998, 633-635.

139 BROWN 1992, 133: „An ideology of silence protected the official triumph of the church.“

140 Soz. h.e. 5,20,7: i் $\gamma \mu \omega ̀ v$ Kapías.

141 Socr. h.e. 3,15; Soz. h.e. 5,11,1-3.

142 So schrieb Augustinus um 400 an die Kurialen von Madaura (ep. 232,3): videtis certe simulacrorum templa partim sine reparatione conlapsa, partim diruta, partim clausa, partim in usus alios commutata, ipsaque simulacra vel confringi vel incendi vel includi vel destrui. Für Antiocheia siehe etwa HAHN 2004, 130-136. 
für den Prätorianerpräfekten oder den Kaiser eine unauffällige Möglichkeit, Unbotmäßigkeit zu sanktionieren. ${ }^{143}$

Betrachtet man nun die überlieferten Konflikte, wie sie vor allem in den Arbeiten von Frank Trombley und Johannes Hahn untersucht worden sind, ${ }^{144}$ so zeichnet sich das folgende Handlungsmuster ab: In vielen Fällen - besonders im ländlichen Raum - agierten Bischöfe, Äbte, Mönche und ihre Anhänger alleine, ohne dass lokale oder kaiserliche Funktionsträger bei ihren gewaltsamen Unternehmungen überhaupt in Erscheinung traten. ${ }^{145} \mathrm{Im}$ städtischen Rahmen handelten dann allerdings die Bischöfe gemeinsam mit kaiserlichen Amtsträgern. Freilich muss berücksichtigt werden, dass diese Konflikte überwiegend von christlicher Warte aus dargestellt sind, welche die Initiative und häufig auch die Ausführung der antipaganen Maßnahmen vornehmlich den kirchlichen Amtsträgern zuschreibt. Wie viele indifferente oder untätige Bischöfe es gegeben hat, können wir zwar nicht mehr ermessen, doch spricht manches dafür, dass nicht wenige von ihnen zumindest noch im 4. Jahrhundert an einem einvernehmlichen Verhältnis zur paganen Bevölkerung(smehrheit) interessiert waren. ${ }^{146}$

Besonders seit den theodosianischen Religionsgesetzen handelten christliche Eiferer bei ihren Maßnahmen gegen den heidnischen Kult in einem für die Heiden repressiven Klima, das ihnen Rückendeckung verschaffte. Auch konnten sie sich auf kaiserliche Konstitutionen berufen, selbst wenn sie mit ihren Handlungen deren Vorgaben überschritten. Um sicher zu gehen, mussten die Bischöfe allerdings nur ein kaiserliches Privileg für ihr Vorgehen gegen den paganen Kult erwirken, indem sie wie Bischof Porphyrius ihre Patronagenetzwerke am kaiserlichen Hof mobilisierten oder wie die afrikanischen Konzilien Gesandtschaften an den Kaiserhof schickten. ${ }^{147}$ Dann konnten sie buchstäblich „das Gesetz als Waffe benutzen“. Porphyrius' Diakon Marcus kehrte aus Constantinopel mit einem agens in rebus zurück, der das kaiserliche Reskript, das heißt ein Privileg, mit

143 Demonstrative Strafmaßnahmen gegen Statthalter sind selten bezeugt: Im Jahr 382 wurde ein dux et praeses Sardiniae, der sich der Erpressung schuldig gemacht hatte, nach seiner Amtszeit sub custodia protectorum in seine Provinz zurückgeführt, um dort vierfachen Ersatz zu leisten. Dies sollte als abschreckendes Beispiel dienen (metus; vgl. Cod. Theod. 9,27 [ad legem Iuliam repetundarum], 3).

144 TROMBLEY 1993-1994; HAHN 2004; vgl. Fowden 1978; VAN DAM 1985; MACMulLEN 1984, 86-101; CHUVIN 1990; LIZZI 1990.

145 Z. B. Sulp. Sev. v. Mart. 13-15 (Gallien); Call. v. Hyp. 30,1 (Bithynien); Soz. h.e. 7,15,13-15 (Apameia); 5,15,5-7 (Kyzikos); vgl. FowDEN 1978, 67-72.

146 Hinweis von Johannes Hahn. Die Haltung des vermeintlich christlichen Bischofs Pelagios von Ilion, der während der Herrschaft des Constantius II. die paganen Kultstätten und -bilder heimlich gepflegt hatte, um sich mit Julians Amtsantritt sofort als Heide erkennen zu geben, dürfte hingegen eher ein Einzelfall gewesen sein, wie auch die Reaktion seiner paganen Glaubensgenossen erkennen lässt (Iul. ep. 79; Bidez-Cumont).

147 Ein Konzil in Carthago hatte z. B. am 16. Juni 401 beschlossen, eine Gesandtschaft an den Kaiser zu schicken de reliquiis idolorum vel templis ab imperatoribus abolendis (Reg. eccl. Carth. exc. 6,58; MUNIER 1974, 196) und de paganorum conviviis auferendis (VI 60; MUNIER 1974, 196). 
Hilfe zweier commentarienses aus dem Statthalterbüro des consularis Palaestinae umsetzte und fast alle Tempel in Gaza schließen ließ. ${ }^{148}$

Die Bischöfe hatten auch in Africa im Jahr 407/8 das Einschreiten des Kaisers provoziert. In seinem Schreiben an den Prätorianerpräfekten wurde zwar vom Prokonsul die Anwendung der leges generales gegen Heiden, Häretiker und Juden verlangt, doch schickte der Prätorianerpräfekt drei seiner agentes in rebus, die kaiserlichen Weisungen auszuführen ${ }^{149}$ - dem Prokonsul und seinem officium misstraute er offensichtlich. Die agentes in rebus hatten nicht die Aufgabe des Prokonsuls zu übernehmen und erhielten auch nicht dessen Vollmachten, sondern sie sollten wie die städtischen Magistrate und die officiales die Delinquenten aufspüren und vor Gericht stellen. ${ }^{150}$ Ein Statthalter konnte in diesem Fall kaum mehr untätig bleiben.

In der Konstitution vom Januar 409, die Honorius an seinen loyalen Prätorianerpräfekten Theodorus gerichtet hatte, der sie dem glaubenseifrigen Prokonsul Donatus zukommen ließ, wird deutlich, dass nur dieser die Religionsprozesse führen durfte. Agentes in rebus zu schicken war nicht nötig, wenn ein Statthalter seine Pflichten ernst nahm. Nötig war freilich die Unterstützung eines iudex durch militärische Befehlshaber, sollte jener mit den Delinquenten nicht fertig werden oder bewaffneter Aufruhr entstehen: So sieht das Gesetz des Honorius vor, dass die Statthalter der afrikanischen Provinzen den comes Africae um Unterstuitzung bitten sollten, wenn bewaffnete Gruppierungen nicht mit Hilfe der örtlichen Magistrate, der Großgrundbesitzer sowie der statthalterlichen officiales vor Gericht gebracht werden könnten. ${ }^{151}$

Anders als bei dem Routinegeschäft der Verurteilung von religiösen Dissidenten betrauten die Kaiser und ihre Prätorianerpräfekten in den Fällen, in denen sie mit Widerstand rechneten, anscheinend von vornherein comites an Stelle der iudices mit der Durchsetzung religionspolitischer Maßnahmen. So wurden im Jahr 399 die comites Africae Gaudentius und Iovius damit beauftragt, in Nordafrika die Heiligtümer zu schließen und die Götterbilder zu zerstören. ${ }^{152}$ Nicht

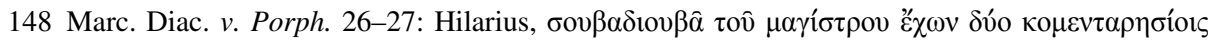

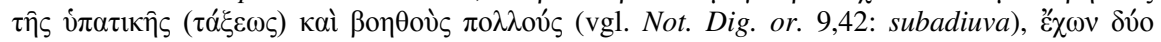

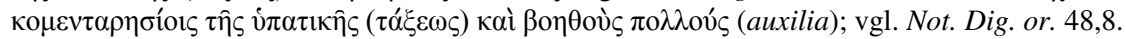

149 Const. Sirmond. 12: nam et agentum in rebus executionem Maximi, Iuliani, Eutychi, ut ea, quae geralibus legibus contra Donatistas, Manichaeos adque huiusmodi haereticos vel gentiles statuta sunt, impleantur, indulsimus.

150 Const. Sirmond. 12: qui tamen scient (agentes in rebus) in omnibus modum statutorum esse servandum, ut ea, quae contra vetitum videntur esse commissa, mox iudicibus iuxta vim legum deferant vindicanda.

151 Const. Sirmond. 14: et si multitudo violenta civilis apparitionis exsecutione et adminiculo ordinum possessorumve non potuerit praesentari, quod se armis aut locorum difficultate tueantur, iudices Africani armatae apparitionis praesidium, datis ad virum spectabilem comitem Africae litteris praelato legis istius tenore, deposcent ut rei talium criminum non evadant.

152 Aug. civ. XVIII 54: in civitate notissima et eminentissima Carthagine Africae Gaudentius et Iovius comites imperatoris Honorii quarto decimo Kalendas Aprilis falsorum deorum templa everterunt et simulacra fregerunt; vgl. Quodvultd.I prom. 3,38,41 (PLRE II 493-494, Gaudenti- 
auszuschließen ist aber, dass Augustinus in seinem Bericht die Beteiligung der örtlichen Statthalter schlicht übergeht. Denn in anderen Fällen wurden comites oder duces von ihren zivilen Kollegen begleitet. So erschienen bei den Unruhen um das Serapeion in Alexandreia im Jahr 392 der praefectus Augustalis gemeinsam mit dem comes Aegypti, ${ }^{153}$ und auch zur Zerstörung des Marneion in Gaza im Jahr 402 kamen der consularis Palaestinae und der dux Palaestinae, die allerdings erst von einem hochrangigen comes consistorianus aus Constantinopel in Bewegung gesetzt wurden, den Bischof Porphyrius als kaiserlichen Sondergesandten mitgebracht hatte. ${ }^{154}$ Könnte man im ersten Fall noch einwenden, dass beide Amtsträger vor Ort residierten und deshalb zugegen waren, ${ }^{155}$ so gilt dies nicht für Gaza, denn beide Funktionsträger kamen aus der Metropolis Caesarea herbei. Die Situation war aber im Folgenden die gleiche: Die Bewohner der Stadt und die städtischen Behörden wurden zusammengerufen, der Militärbefehlshaber und die Soldaten standen bereit, um Aufruhr im Keim zu ersticken, und der Statthalter ließ das kaiserliche Dekret verlesen, das anschließend in die Tat umgesetzt wurde. $^{156}$

In den übrigen überlieferten Fällen gingen comites oder duces ohne erkennbare Begleitung ziviler Amtsträger gegen pagane Kultstätten vor: Neben den bereits erwähnten comites Africae Gaudentius und Iovius waren dies der comes Orientis Acacius bei der Zerstörung des Altars an der Eiche Mamre und der Reinigung des Temenos unter Constantin, ${ }^{157}$ der dux Aegypti Artemius bei der Zerstörung zahlreicher Götterbilder unter Constantius II., ${ }^{158}$ der dux Osrhoenae Palladius bei der

us 5; II 622-623, Iovius 2). Dies führte hier und dort zum Aufruhr wie bei der Zerstörung einer Hercules-Statue in der Colonia Aurelia Sufetana (Aug. ep. 50).

153 Eun. vit. soph. 472; Rufin. h.e. 9,22-23; Socr. h.e. 5,16; Soz. h. e. 7,15; Thdt. h.e. 5,22; zu den Vorgängen FOWDEN 1978, 69-71; TROMBLEY 1993, I 129-145; HAHN 2004, 81-94.

154 Marc. Diac. v. Porph. 51: Cynegius (vielleicht ein Verwandter des Maternus Cynegius; vgl.

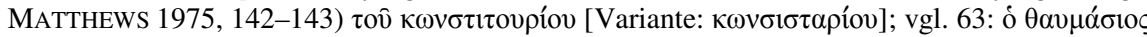

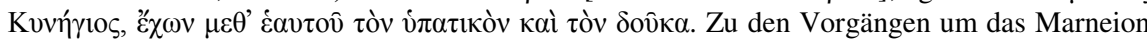
FOWDEN 1978, 72-76; VAN DAM 1985; TROMBLEY 1993, I 207-222; HAHN 2004, 209-222.

155 Wie die zivilen Machthaber in der Metropolis Alexandreia auf den Aufruhr reagierten, erzählt Rufinus (h.e. 11,22; p. 1026, Z. 4-8 Mommsen): at hi, quibus Romanorum legum custodia iurisque dicendi cura permissa est, cognitis quae gesta fuerant, turbati atque perterriti ad templum (Serapidis) convolant, causas audaciae percontantur, et quid sibi vellet ille concursus, in quo cum tanto scelere ante aras sanguis civium funderetur, inquirunt. Als sie der Lage nicht Herr wurden, weil sich die Aufrührer im Tempel verschanzt hatten, res gesta ad imperatorem refertur (Z. 13).

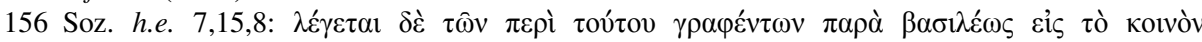

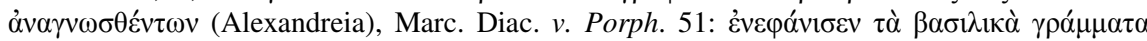
(Gaza).

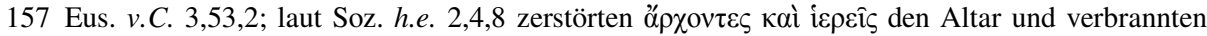
die Kultstatuen. Acacius war comes in Palästina 326-330 (PLRE I 6, Acacius 4).

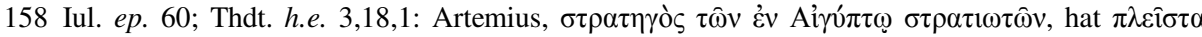

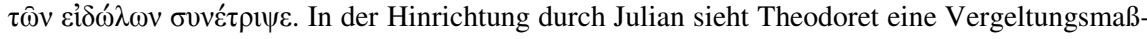
nahme, während Amm. 22,11,2 allgemein von atrocia crimina spricht, deren ihn die Alexandriner angeklagt hatten; vgl. PLRE I 112, Artemius 2; HAHN 2004, 174. 
Profanierung der Götterbilder im Tempel von Edessa im Jahr 382, ${ }^{159}$ der anonyme comes Orientis beim Fällen der Zypressen im Hain von Daphne um das Jahr $388^{160}$ und der ungenannte comes Orientis oder praefectus praetorio Orientis ${ }^{161}$ bei der versuchten Zerstörung des Zeustempel in Apameia im Jahr 386. ${ }^{162}$ Maternus Cynegius ist der einzige Prätorianerpräfekt, von dem die Überlieferung berichtet, dass er selbst gewaltsame Maßnahmen gegen pagane Heiligtümer nicht nur anordnete, sondern auch in seiner Gegenwart ausführen ließ. ${ }^{163}$

Der Befund der wenigen erzählenden Quellen zur Rolle der Gouverneure beim Vorgehen gegen die Heiden ist nur schwer zu verallgemeinern. Wegen der perspektivischen Verzerrung der christlichen Historiographie müssen wir damit rechnen, dass Statthalter schlicht nicht erwähnt wurden, weil sie in der Dramaturgie der Konflikte keinen Platz fanden. Vor dem Hintergrund der Konstitutionen wird deutlich, dass sich die Vorwürfe statthalterlicher desidia, neglegentia und dissimulatio auf verschiedene Aufgabenbereiche beziehen. Einerseits haben sich die Gouverneure offenbar nur selten beim Aufspüren religiöser Dissidenten, besonders heidnischer, hervorgetan. Sie überließen dies, wo nicht ihre officiales oder die städtischen Kurialen tätig wurden, Bischöfen oder Mönchen. ${ }^{164}$ In besonders heiklen Fällen wurden mit dieser Inquisition agentes in rebus beauftragt. Die Statthalter waren anscheinend auch nicht besonders daran interessiert, die religionspolitischen Gesetze anzuwenden, wenn mit Widerstand zu rechnen war. Der Prätorianerpräfekt schickte deshalb lieber höherrangige comites, obwohl die Gouverneure selber über kleinere Truppenkontingente verfügten. ${ }^{165}$ Schließlich haben

159 Cod. Theod. 16,10,8 (a. 382) an den dux Osdroenae Palladius (PLRE I 660, Palladius 11). Dieses oraculum ist ein Privileg, das nur für diesen Tempel gilt. Es ordnet die Offenhaltung des Tempels zum profanen Gebrauch für die Bevölkerung an, wobei die simulacra als Kunstwerke, nicht als Objekte der Verehrung, zu betrachten seien.

160 Lib. or. 1,255; 262-266; or. 30,42-43; PLRE I 1015, Anonymus 61; vgl. Cod. Theod. 10,1,12; Cod. Iust. XI 78 [de cupressis ex lucu Daphnensi], 2.

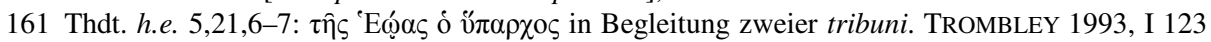
geht vom praefectus praetorio Orientis Maternus Cynegius aus (vgl. Lib. or. 30,44-47), ebenso MATTHEWS 1975, 140-144; Paschoud im Kommentar zu Zos. 4,37,3 (p. 424-426); WIEMER 1995, 123-124; skeptisch FOWDEN 1978, 62-64. ט゙ $\alpha \rho \chi o \varsigma$ ist sowohl ein gängiges Äquivalent für praefectus als auch als Titel für den comes Orientis belegt.

162 Auf Betreiben des Bischofs Marcellus: Soz. h.e. 7,15,12-15; vgl. FowdEN 1978, 64-67; TROMBLEY 1993, I 126-128. Marcellus zerstörte auch Tempel im Umland von Apameia und

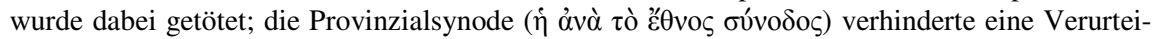
lung der Mörder.

163 Cynegius wurde von Theodosius unter anderem mit dem Auftrag in die Diözesen Orient und

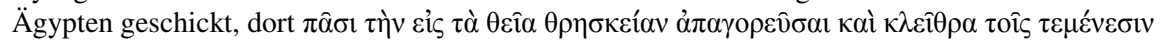

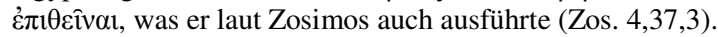

164 BROWN 1963, 293 vertritt mit Bezug auf Nordafrika sogar die These, dass die kaiserliche Religionsgesetzgebung nur dort wirksam war, wo es eine starke nizänische Gemeinde gab.

165 Als wenige Jahre nach der Zerstörung des Marneion Unruhen zwischen Heiden und Christen in Gaza ausbrachen, schickte der consularis Palaestinae Clarus zwei seiner commentarienses mit zahlreichen Soldaten, die den Aufruhr erstickten; vgl. Marc. Diac. v. Porph. 99 (a. 407); Clarus: PLRE II 298. 
Statthalter häufiger auch ihre Pflichten als iudices ordinarii in Religionsprozessen nicht im Sinne der kaiserlichen Direktiven erfüllt. Dies ist freilich kein Spezifikum der Religionsprozesse.

Dieser Befund erlaubt folgende Schlussfolgerung: Sowohl die Bischöfe als auch die Kaiser waren offenbar bestrebt, solche heiklen Aufgaben Funktionsträgern zu übergeben, die einerseits rangmäßig möglichst über den Gouverneuren standen und andererseits nicht so tief in das Geflecht der provinzialen Netzwerke und Feindschaften involviert waren wie die iudices, die sich notwendigerweise für ihre täglichen Geschäfte mit lokalen Gruppierungen verbinden mussten. Das führt zur Frage nach den Bedingungen, unter denen ein Gouverneur in seiner Provinz die kaiserlichen Konstitutionen durchsetzen sollte.

\section{Die Ohnmacht des Gouverneurs in der provinzialen Figuration}

Die Strafbestimmungen in den Konstitutionen benennen diejenigen provinzialen Ranggruppen, die sich der Umsetzung der kaiserlichen Religionspolitik immer wieder entzogen oder widersetzt haben. Dies sind neben dem Gouverneur selbst die officiales aus dem Statthalterbüro, die defensores civitatis, die curiales, aus denen sich die principales oder primates civitatis als provinziale Elite hervorhoben, die kaiserlichen procuratores, die possessores und schließlich die honora$t i$, die hohe Ämter am Hof oder im Reichsdienst bekleidet und sich nun in die Provinz zurückgezogen hatten. ${ }^{166} \mathrm{Zu}$ den maßgeblichen Gruppen der Provinz, welche die Religionspolitik in der Regel unterstützten, gehörten neben den Bischöfen und den militärischen Amtsträgern auch die kaiserlichen Inspektoren (agentes in rebus, notarii und tribuni), die den Gouverneur kontrollieren sollten. Sich in diesem je nach Ort und Zeitpunkt spezifischen Geflecht konfligierender Interessen auch nur zu behaupten, geschweige denn sich durchzusetzen, war für die iudices schon bei den alltäglichen Geschäften nicht einfach, ganz zu schweigen von politisch brisanten Situationen wie den Religionskonflikten. Ich werde im Folgenden den statthalterlichen Handlungsspielraum knapp skizzieren. Die zu beschreibende provinziale Konstellation kann als Figuration bezeichnet werden, in der das Geflecht der Abhängigkeiten den Spielraum und die Grenzen der einzelnen Akteure innerhalb ihrer gesellschaftlichen Positionen bestimmte. ${ }^{167}$ Die rhetorisch so wirksam beklagte mala desidia iudicum war ein latentes strukturel-

166 Cod. Theod. 16,10,12,4 (a. 392); 16,10,13 (a. 395); Const. Sirmond. 12 und 14; zur Rangdifferenzierung vgl. Cod. Theod. 16,5,52 (a. 412); 16,5,54 (a. 414).

167 Vgl. oben Anm. 24. Für eine Charakteristik der spätantiken Statthalterschaft CoRCORAN 1996, 234-253; LENDON 1997, 212-235; CARRIÉ 1998; zusammenfassend PALME 1999, 98-100; dazu auch BROWN 1992, 3-34. Aus provinzialer Perspektive für Cappadocia VAN DAM 2002, 77-94, für Ägypten BAGNALL 1993, 62-67, zum kaiserzeitlichen Hintergrund MEYER-ZwIFFELHOFFER 2002. 
les Element der provinzialen Figuration, das unter bestimmten Umständen wie den hier erörterten religiösen Konflikten besonders virulent werden konnte.

1. Es ist deutlich geworden, dass die Hauptaufgabe der spätantiken Statthalter, für den Einzug der Steuern und die Rechtsprechung sowie für die Aufrechterhaltung der öffentlichen Ordnung zu sorgen, durch die kaiserliche Religionspolitik gegenüber Heiden und Häretikern immer wieder konterkariert wurde. Schon die Konstitutionen waren das Resultat von religiösen Auseinandersetzungen innerhalb der Provinz, die nun durch die kaiserlichen Direktiven angeheizt wurden, sollte sie der Gouverneur umsetzen wollen. Dabei ging es ja keineswegs nur um die ,Intoleranz ' gegenüber einer diskriminierten religiösen Praxis, sondern vor allem um die umfangreichen Konfiskationen und Vermögensstrafen einerseits, sowie um die soziale Ächtung der Dissidenten durch den Entzug von honores, dignitates und bürgerlichem Status andererseits. Auf dem Spiel standen quies publica und publica disciplina. Die Anweisungen, ländliche Heiligtümer oder Synagogen zu zerstören, wo dies ohne Aufruhr möglich sei, zeigt, dass mit seditiones vor allem in den Städten zu rechnen war, besonders den Metropoleis, in denen die kaiserlichen und provinzialen Amtsträger residierten; Alexandreia und Antiocheia legen davon ein beredtes Zeugnis ab. Dabei konnte selbst das Leben des Gouverneurs in Gefahr geraten, so als ein consularis Syriae Primae nach der Verlesung eines kaiserlichen Ediktes im Jahr 387 in seinem praetorium in Antocheia angegriffen wurde oder der praefectus Augustalis Orestes in Alexandreia im Jahr 415 bei den Auseinandersetzungen mit dem Bischof Kyrill um die Vertreibung der Juden beinahe gesteinigt worden wäre. ${ }^{168}$ Libanios nennt das Theater von Antiocheia sogar ein „Schlachtfeld im Kampf gegen die Statthalter“. ${ }^{169}$ Und als sich Abt Schenute von Atripe zu Beginn des 5. Jahrhunderts nach der Zerstörung eines Pantempels und eines Kronosheiligtums in Panopolis vor dem dux Thebaidos Theodosius verantworten sollte, stachelte jener die Menge so auf, dass sich das Tribunal plötzlich gegen den $d u x$ richtete. ${ }^{170}$

Da die Religionsgesetze zumindest noch im 4. Jahrhundert in vielen Fällen die örtliche Bevölkerung spalteten, liegt die Vermutung nahe, dass die meisten iudices kein großes Interesse an ihrer Erzwingung gehabt haben können. Natürlich gab es hier und da auch fanatische Statthalter, besonders gegenüber Christen in den Jahren, als die Maßnahmen Julians polarisierten. ${ }^{171}$ Die Überlieferung zeigt zudem, dass sich christliche Statthalter weniger von paganen als von häretischen Gruppierungen, die ihre orthodoxe Identität bedrohen konnten, herausgefordert sahen. In Analogie zu den kaiserzeitlichen Christenverfolgungen ${ }^{172}$ liegt die Annahme auf der Hand, dass die meisten iudices eher gezwungenermaßen und reaktiv tätig wurden, zumal die eigentliche Untersuchung eines crimen sacrilegii oder

168 Lib. or. 30,3 und Socr. h.e. 7,14; PLRE II 810-811, Orestes 1.

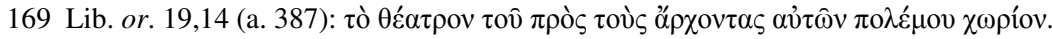

170 HAHN 2004, 256-257; PLRE II 1101, Theodosius 10.

171 DRAKE 1996, 33-35; FOWDEN 1998, 543-548.

172 MEYER-ZWIFFELHOFFER 2002, 143-171. 
publicum crimen vor Ort von den städtischen Magistraten und officiales vorgenommen wurde. Nur am Statthaltersitz selbst, in der Metropolis, waren die Gouverneure mit den Vorgängen direkt konfrontiert, wie das Beispiel Alexandreias zeigt.

2. Es gibt einen weiteren Grund, weshalb sich viele iudices wohl nicht allzu sehr für die kaiserliche Religionspolitik eingesetzt haben dürften: Sie waren, anders als ihr officium, die Bischöfe, die Kurialen oder die honorati, nur ein oder zwei Jahre vor Ort im Amt und darauf angewiesen, ein günstiges Zeugnis für ihre Amtsverwaltung zu erhalten, wollten sie weiter Karriere machen. Die so genannte ,Autobiographie' des Libanios (or. 1) zeigt in aller Klarheit, wie vor dem Auge eines ansässigen Notabeln die Statthalter Jahr für Jahr vorbeizogen, und wie kritisch ihre Tätigkeit wahrgenommen wurde. Liest man die Reden und Briefe von Libanios oder Synesios, waren die meisten von ihnen ausschweifend, grausam, desinteressiert und korrupt. ${ }^{173}$ Dies kann freilich so nicht zugetroffen haben, denn die Gouverneure wurden nach dem Ende ihrer Amtszeit beurteilt. Bereits Constantin hatte mit einem Edikt die Provinziallandtage aufgefordert, die Amtsführung ihrer Statthalter öffentlich zu akklamieren oder sich bei den Vikaren und Prätorianerpräfekten über sie zu beklagen. ${ }^{174}$ Neben diesen förmlichen Zeugnissen konnte der Statthalter auch brieflich oder mit einer Rede belobigt werden. Augustinus etwa hatte dem proconsul Africae Donatus im Jahr 409 einen solchen Brief geschrieben; ob dieser auch an offizielle Stellen ging, ist nicht klar. Und Synesios stellte dem dux Libyae Superioris Marcellinus im Jahr 413 ein Zeugnis $(\mu \alpha \rho \tau v \rho i ́ \alpha)$ aus, das er aber dem örtlichen Statthalter schickte. ${ }^{175}$ Dass diese dem Prätorianerpräfekten oder dem Kaiser zur Kenntnis gebrachten Beurteilungen keineswegs nur momentane Bedeutung besaßen, lehrt in den 430er Jahren die Praxis des proconsul Africae, die Liste der bisherigen Amtsinhaber coram populo vorlesen zu lassen, damit diese akklamiert oder geschmäht werden konnten. Die Statthalter gingen also auch in das provinziale Gedächtnis ein. ${ }^{176}$ Am dauerhaftes-

173 Der locus classicus ist Libanios' Rede gegen den consularis Syriae Primae Tisamenus im Jahr 386 (or. 33; vgl. or. 45); dazu CARRIÉ 1998, 25-27. Siehe auch Lib. or. 50, 18-19 (a. 384).

174 Cod. Theod. 1,16,6,1 ad provinciales (= Cod. Iust. I 40,3; a. 331): iustissimos autem et vigilantissimos iudices publicis adclamationibus conlaudandi damus omnibus potestatem, ut honoris eis auctiores praeferamus processus, e contrario iniustis et maleficis querellarum vocibus accusandis, ut censurae nostrae vigor eos absumat; nam si verae voces sunt nec ad libidinem per clientelas effusae, diligenter investigabimus, praefectis praetorio et comitibus, qui per provincias constituti sunt, provincialium nostrorum voces ad nostram scientiam referentibus. Ein solches Lob ist aus Ephesos inschriftlich erhalten (I. Ephesos 44; a. 440).

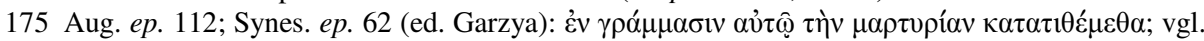
SCHMITT 2001, 602-603. Demnach erhielten auch militärische Befehlshaber derartige

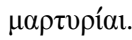

176 Quodvultd. I prom. 13,15 (a. 436): in calculis eburneis nomina proconsulum conscripta Karthaini in foro coram populo a praesenti iudice sub certis vocabulis citabantur et erat sollemnis dies albi citatio. Hi qui avaritiam superantes rem publicam fideliter egerant, suffragiis favoribusque etiam absentes honorabantur. Eos vero quos rapacitas vicerat, populus conviciis sibilisque notabat. 
ten kündeten freilich die immer noch zahlreichen Ehrenmonumente, wo nicht von guter Amtsverwaltung, so doch von so großzügigen Spenden für die Reparatur und Errichtung städtischer Bauwerke, dass sich der Kaiser genötigt sah, den Aufwand für neue Bauwerke zu beschränken. ${ }^{177}$

Auf der anderen Seite war ein Gouverneur jedoch Pressionen ausgesetzt. Als ein christlicher comes Orientis im Jahr 388 damit begann, die Zypressen im Hain von Daphne abzuschlagen, ließ ihn Libanios warnen: Er werde dem Kaiser darüber berichten, der diesen Hain bereits mit einem Gesetz geschützt hatte. ${ }^{178}$ Dass dies nicht nur in den großen Reichsmetropolen möglich war, in denen eine hochrangige provinziale Gesellschaft residierte wie in Antiocheia oder Alexandreia, lehrt das Beispiel Kyrenes: Synesios betrieb mit seinen provinzialen Bischofskollegen im Jahr 411 die Exkommunikation und Ablösung des praeses Libyae superioris Andronicus. ${ }^{179}$ Selbst wenn ein missliebiger Gouverneur seine Amtszeit überstand und keine weiteren Ambitionen hatte oder vorübergehend pausieren musste, hatte er, sobald er sich als privatus auf seine Güter zurückzog, die Rache seiner Feinde zu gewärtigen. So drohte Libanios im Jahr 389 dem consularis Syriae Eutropios: „Ich [...] sehe dich zittern und dich selbst verbannen und zum Aufenthalt eine Stadt suchen außerhalb Syriens, das du verwaltet hast. So gut hast du es also verwaltet!" 180

3. Ein letztes Moment lässt sich mit einer treffenden Formulierung von John E. Lendon so ausdrücken: „Under-honourable governors“ waren mit „overhonourable subjects“ konfrontiert. ${ }^{181}$ Auch dies traf nicht nur auf die großen Metropolen zu; allein in Timgad etwa war der consularis Numidiae im Jahr 362 mit zehn lokalen clarissimi konfrontiert, wovon fünf als Patrone der Stadt fungierten. ${ }^{182}$ Dabei spielte - abgesehen von der niederen Herkunft mancher Statthal$\operatorname{ter}^{183}$ - der Konflikt zwischen dem Ranggefüge der provinzialen Gesellschaft einerseits sowie den Amtspflichten des Gouverneurs andererseits eine zentrale Rolle. Generell galt zwar der Grundsatz der Ehrerbietung (reverentia) von Amtsträgern geringeren Ranges ranghöheren ehemaligen Magistraten gegenüber, doch stand dagegen das öffentliche Wohl (publica utilitas), insofern der Statthalter als

177 ROBerT 1948; Horster 1998; vgl. Cod. Theod. 15,1 [de operibus publicis], 20 (a. 380 an den praefectus Aegypti): iudex, qui ad provinciam fuerit destinatus, duas partes vel incuria vel vetustate conlabsas ad statum pristinum nitoris adducat adque tertiam construat novitatis, si tamen famae et propriis cupit laudibus providere. Ein Beispiel bietet Lib. ep. 852: der comes Orientis Proclus baute Strassen, Portiken, Bäder, Märkte.

178 Lib. or. 1,255; 262-266; Cod. Theod. 10,1 [de iure fisci], 12 (a. 379); Cod. Iust. 11,78 [de cupressis ex lucu Daphnensi], 1-2.

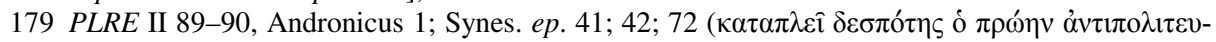

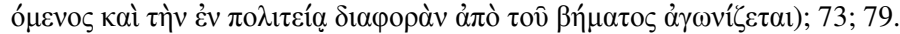

180 Vgl. Lib. or. 4,39 gegen den consularis Syriae Eutropius (PLRE I 318, Eutropius 3).

181 LENDON 1997, 223; vgl. schon JONES 1986, 502-504.

182 Chastagnol 1978, 22-28.

183 Etwa die Auseinandersetzung des Bischofs Synesios von Kyrene mit dem praeses Andronicus (ep. 41). 
iudex von Rangrücksichten theoretisch befreit war. ${ }^{184}$ Dass dies praktisch aber eine Frage der Macht und des Einflusses war, zeigen Bestimmungen wie diejenige, der Gouverneur dürfe einen Prozess direkt an die nächste Instanz verweisen, wenn sein Gegner zu mächtig sei. ${ }^{185}$

Wie prekär diese Situation war, zeigt der ordo salutationis des consularis Numidiae Ulpius Mariscianus aus der Zeit Iulians, der die provinziale Gesellschaft der honestiores in der numidischen Hauptstadt Constantine in feinabgestuften Ranggruppen abbildete. ${ }^{186}$ In einer Metropolis wie Alexandreia, Antiocheia, Carthago oder Ephesos war der Statthalter freilich noch mit weitaus höherrangigen Personen als in Numidia konfrontiert. Aus dem ordo salutationis geht hervor, dass das Privileg der salutatio und damit des ius osculi - auch gegen den Willen des Gouverneurs - allen Amtsträgern oder honorati ab der Würde von clarissimi zustand. Den gleichen Ranggruppen wurde auch das Privileg des freien Zugangs in das secretarium des Gouverneurs (ingressus) und des Rechtes, in seinem consilium zu sitzen (consessus) verliehen. ${ }^{187}$ Welcher Druck dabei auf den Statthaltern lasten konnte, machen zwei Reden des Libanios deutlich, ${ }^{188}$ der selbst seine Einflussmöglichkeiten auf die Gouverneure hervorhob, obwohl er nicht einmal einen offiziellen Rang besaß, sondern nur sein Ansehen als Redner aus einer alten Kurialenfamilie in die Waagschale werfen konnte. ${ }^{189}$

Zwar versuchten die Kaiser immer wieder, mit Gesetzen die provinzialen Notabeln und honorati zu zwingen, einen niederrangigeren, aber mit Amtsbefugnissen ausgestatteten Gouverneur zu respektieren, ${ }^{190}$ anscheinend aber erfolglos.

184 Cod. Iust. 1,40,5 (a. 364): potioris gradus iudicibus ab inferioribus competens reverentia tribuatur. Sed ubi publica tractatur utilitas, etsi minor iudex veritatem investigaverit, nulla maiori inrogatur iniuria.

185 Cod. Theod. 1,16,4 = Cod. Iust. 1,40,2 (a. 328?): praesides provinciarum oportet, si quis potiorum extiterit insolentior et ipsi vindicare non possunt aut examinare aut pronuntiare nequeunt, de eius nomine ad nos aut certe ad gravitatis tuae (Prätorianerpräfekt) scientiam referre, quo provideatur, qualiter publicae disciplinae et laesis minoribus consulatur. Vgl. unten Anm. 189.

186 CHASTAGNOL 1978, 75-81.

187 Cod. Theod. 6,18 [de comitibus vacantibus], 1 (a. 412): nullam honorabiles viri in publicis salutationibus patiantur iniuriam; 6,26 [de proximis, comitibus dispositionum ceterisque, qui in sacris scriniis militant], 5 (a. 389): in sacris scriniis nostris militantes ordinarii iudices a salutatione non arceant, etiam inviti in consessum accipiant; 6,26,16 (a. 410): consedendi ingrediendique secretarium licentia; vgl. LENDON 1997, 177-181.

188 Lib. or. 51 und 52.

189 Lib. or. 2,7-8 (a. 380/81). WIEMER 1995 hat überzeugend gegen die communis opinio dargelegt, dass Libanios zu keiner Zeit eine honoraria dignitas als praefectus praetorio oder quaestor sacri palatii erhalten hatte. Das bei Libanios genannte $\gamma \rho \alpha \mu \mu \alpha \tau \varepsilon i ̂ o v$ bezieht er auf eine von Julian angebotene, aber von Libanios ausgeschlagene ex comitibus dignitas (113).

190 Cod. Theod. 1,15 [de officio vicarii], 1 (a. 325): Solche Rechtsfälle dürfen unter Umgehung des iudex vor den vicarius gebracht werden, in quibus persona potentior inferiorem aut minorem iudicem premere potest; Cod. Theod. 12,1 [de decurionibus], 150 (a. 395): curiales, qui honorariam adepti sunt comitivam, formidare debent eos, quorum sunt moderationi commissi nec se existimare ideo meruisse dignitatem, ut iudicum praecepta despiciant. 
Doch war dieser nicht nur den honorati unterlegen, ${ }^{191}$ sondern in Konfliktsituationen auch Pressionen seines officium, besonders des dort tätigen agens in rebus, ausgeliefert. Dass schließlich die Bischöfe ein gefährlicher Gegner sein konnten, lehrt das Beispiel des Synesios ebenso wie die reichsweiten Verbindungen des Porphyrios von Gaza.

In der Frage der Durchsetzung der kaiserlicher Religionspolitik gegenüber den Heiden wie auch den Häretikern kann also zu Recht von mala desidia iudicum gesprochen werden. Die Position der Statthalter in der provinzialen Figuration war zu prekär, ihre Ohnmacht angesichts dauerhafter Netzwerke höherrangiger Kontrahenten zu groß, als dass man von ihnen besonderes Engagement bei derart unruheträchtigen Vorhaben hätte erwarten dürfen. Aus diesem Grund sind sie kaum je von den Kaisern mit entsprechenden Missionen beauftragt worden, und auch die Bischöfe wandten sich lieber an besser positionierte Fürsprecher, die nicht so sehr in die provinzialen Beziehungsgeflechte verstrickt waren. Hatten allerdings andere die Initiative bei der Unterdrückung paganer Kulte in den Städten oder in der Chora übernommen, mussten sie als iudices die Folgen dieser Politik strafrechtlich wie zivilrechtlich verwalten. Insofern kann man mit den Worten Peter Browns schließen: „The failure of the emperors to impose their religious policies on large regions of the empire is a measure of the silent powers of resistence of which a late Roman provincial society remained capable.“192

\section{Bibliographie}

Bagnall, R.S., Egypt in Late Antiquity, Princeton 1993.

Barnes, T.D., Statistics and the Conversion of the Roman Aristocracy, JRS 85 (1995), 135-147.

Boyarin, D., The Christian Invention of Judaism: The Theodosian Empire and the Rabbinic Refusal of Religion, Representations 85 (2004), 21-57.

Bradbury, S., Constantine and the Problem of Anti-Pagan Legislation in the Fourth Century, CPh 89 (1994), 120-139.

Bradbury, S. (Hrsg.), Severus of Minorca. Letter on the Conversion of the Jews, Oxford 1996.

Brown, P., Religious Coercion in the Later Roman Empire: The Case of North Africa, History 48 (1963), 283-305.

Brown, P., Power and Persuasion in Late Antiquity: Towards a Christian Empire, Madison 1992.

Brown, P., Christianization and Religious Conflict, in: Cameron, A. - Garnsey, P. (Hrsg.), The Cambridge Ancient History, Bd. XIII: The Late Empire, A.D. 337-425, Cambridge ${ }^{2} 1998,632-664$.

191 Cod. Theod. $1,16,13$ (a. 377).

192 BROWN 1992, 23. 
Carrié, J.-M., Le gouverneur romain à l'époque tardive. Les directions possibles de l'enquête, AnTard 6 (1998), 17-30.

Chastagnol, A., L'album municipal de Timgad, Bonn 1978.

Chuvin, P., A Chronicle of the Last Pagans, Cambridge, MA - London 1990 [franz. Paris 1990].

Corcoran, S. The Empire of the Tetrarchs: Imperial Pronouncements and Government A.D. 284-324, Oxford 1996.

Curran, J.R., Pagan City and Christian Capital: Rome in the Fourth Century, Oxford 1999.

Drake, H.A., Lambs into Lions: Explaining Early Christian Intolerance, P\&P 153 (1996), 3-36.

Elias, N., Soziologie und Geschichtswissenschaft, in: ders., Die höfische Gesellschaft, Darmstadt - Neuwied ${ }^{2} 1975,9-59$.

Emmel, S., From the Other Side of the Nile: Shenute and Panopolis, in: Egberts, A. Muhs, B.P. - Vliet, J. van der (Hrsg.), Perspectives on Panopolis: An Egyptian Town from Alexander the Great to the Arab Conquest, Leiden - Boston - Köln 2002, 95113.

Enßlin, W., Die Religionspolitik des Kaisers Theodosius d. Gr., in: SBAW, phil.-hist. Kl., München 1953, Heft 2.

Errington, R.M., Christian Accounts of the Religious Legislation of Theodosius I, Klio 79 (1997), 398-443.

Fögen, M.T., Die Enteignung der Wahrsager. Studien zum kaiserlichen Wissensmonopol in der Spätantike, Frankfurt a. M. 1997.

Fowden, G., Bishops and Temples in the Eastern Roman Empire A.D. 320-435, JThS 29 (1978), 53-78.

Fowden, G., Polytheist Religion and Philosophy, in: Cameron, A. - Garnsey, P. (Hrsg.), The Cambridge Ancient History, Bd. XIII: The Late Empire, A.D. 337-425, Cambridge $21998,538-560$.

Hahn, J., Gewalt und religiöser Konflikt. Studien zu den Auseinandersetzungen zwischen Christen, Heiden und Juden im Osten des Römischen Reiches (von Konstantin bis Theodosius II.) (Klio-Beihefte n. F. 8), Berlin 2004.

Hahn, J. - Emmel, St. - Gotter, U. (Hrsg.), From Temple to Church: Destruction and Renewal of Local Cultic Topography in Late Antiquity (Religions in the GraecoRoman World 163), Leiden 2008

Harl, K.W., Sacrifice and Pagan Belief in Fifth- and Sixth-Century Byzantium, P\&P 128 (1990), 7-27.

Harries, J., The Background to the Code, in: Harries, J. - Wood, I. (Hrsg.), The Theodosian Code: Studies in the Imperial Law of Late Antiquity, London 1993, 1-16.

Harries, J., Law and Empire in Late Antiquity, Cambridge 1999.

Honoré, T., Law in the Crisis of Empire 379-455 A.D.: The Theodosian Dynasty and Its Quaestors, Oxford 1998.

Horster, M., Ehrungen spätantiker Statthalter, AnTard 6 (1998), 37-59.

Hunt, D., Christianizing the Roman Empire: The Evidence of the Code, in: Harries, J. Wood, I. (Hrsg.), The Theodosian Code: Studies in the Imperial Law of Late Antiquity, London 1993, 143-158.

Jones, A.H.M., The Later Roman Empire, 284-602: A Social, Economic, and Administrative Survey, Norman 1964 [ND Baltimore 1986].

Kaden, E.-H., Die Edikte gegen die Manichäer von Diokletian bis Justinian, in: Gerwig, M. - Simonius, A. (Hrsg.), Festschrift Hans Lewald, Basel 1953, 55-68. 
Lendon, J.E., Empire of Honour: The Art of Government in the Roman Empire, Oxford 1997.

Lepelley, C., L'administration des provinces d'Afrique avant la conquête vandale, AnTard 10 (2002), 61-72.

Leppin, H., Constantius II. und das Heidentum, Athenaeum 87 (1999), 457-480.

Lizzi, R., Ambrose's Contemporaries and the Christianization of Northern Italy, JRS 80 (1990), 156-173.

MacMullen, R., Christianizing the Roman Empire (A.D. 100-400), New Haven - London 1984.

Markus, R.M., The End of Ancient Christianity, Cambridge 1990.

Matthews, J., Western Aristocracies and Imperial Court, A.D. 364-425, Oxford 1975.

Matthews, J., The Making of the Code, in: Harries, J. - Wood, I. (Hrsg.), The Theodosian Code: Studies in the Imperial Law of Late Antiquity, London 1993, 19-44.

Matthews, J., Laying Down the Law: A Study of the Theodosian Code, New Haven London 2000.

Merkelbach, R. - Stauber, J. (Hrsg.), Steinepigramme aus dem griechischen Osten, Bd. I: Die Westküste Kleinasiens von Knidos bis Ilion, Stuttgart 1998.

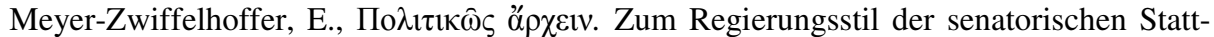
halter in den kaiserzeitlichen griechischen Provinzen (Historia Einzelschriften 165), Stuttgart 2002.

Mommsen, T., Römisches Strafrecht, Leipzig 1899.

Munier, C. (Hrsg.), Concilia Africae, a. 345 - a. 525 (Corpus Christianorum, Series Latina 149), Turnhout 1974.

Palme, B., Die officia der Statthalter in der Spätantike. Forschungsstand und Perspektiven, AnTard 7 (1999), 85-133.

Robert, L., Épigrammes relatives à des gouverneurs, in: ders., Hellenica, Bd. IV: Épigrammes du Bas-Empire, Paris 1948, 35-114.

Rosen, K., Iudex und officium: Kollektivstrafe, Kontrolle und Effizienz in der spätantiken Provinzialverwaltung, AncSoc 21 (1990), 273-291.

Roueché, C., Aphrodisias in Late Antiquity, London 1989.

Salzman, M.R., Superstitio in the Codex Theodosianus and the Persecution of Pagans, VChr 41 (1987), 172-188.

Santalucia, B., Diritto e processo penale nell' antica Roma, Mailand ${ }^{2} 1998$ (1. Aufl. 1988).

Schmitt, T., Die Bekehrung des Synesios von Kyrene. Politik und Philosophie, Hof und Provinz als Handlungsräume eines Aristokraten bis zu seiner Wahl zum Metropoliten von Ptolemaïs, München - Leipzig 2001.

Trombley, F.R., Paganism in the Greek World at the End of Antiquity: The Case of Rural Anatolia and Greece, HThR 78 (1985), 327-352.

Trombley, F.R., Hellenic Religion and Christianization c. 370-529 (Religions in the Graeco-Roman World 115), 2 Bde., Leiden 1993-1994.

van Dam, R., From Paganism to Christianity at Late Antique Gaza, Viator 16 (1985), 120.

van Dam, R. Kingdom of Snow: Roman Rule and Greek Culture in Cappadocia, Philadelphia 2002.

Veyne, P., Clientèle et corruption au service de l'état: la venalité des offices dans le BasEmpire romain, Annales ESC 36 (1981), 339-360. 
Wiemer, H.-U., Die Rangstellung des Sophisten Libanios unter den Kaisern Julian, Valens und Theodosius. Mit einem Anhang über Abfassung und Verbreitung von Libanios' Rede Für die Tempel (Or. 30), Chiron 25 (1995), 89-130. 



\title{
Zwischen Christentum und Staatsraison. Römisches Imperium und religiöse Gewalt ${ }^{*}$
}

\author{
ULRICH GOTTER
}

Im Jahre 388 kam es in Kallinikon am Euphrat, als man gerade das Fest der Makkabäischen Brüder beging, zu Ausschreitungen. Was damals genau und in welcher Reihenfolge geschah, ist leider alles andere als transparent. Sicher erscheint immerhin, daß der dortige Bischof Mönche zur Gewaltanwendung angestiftet, den städtischen Mob mobilisiert und auf die Kultplätze Andersgläubiger losgelassen hat. Das Ergebnis waren Krawalle sowie der Brand der örtlichen Synagoge und eines Konventikels der Valentinianer, einer gnostischen Sekte. ${ }^{1}$ Der Zeitpunkt für die Aktion war gut gewählt und wohl kaum vom Zufall diktiert: Die ,Makkabäischen Brüder` waren, gemäß der Überlieferung, jüdische Märtyrer aus dem Makkabäerkrieg, deren wichtigste Verehrungsstätte die Christen von Antiocheia wohl kurz zuvor der jüdischen Gemeinde entwunden hatten. ${ }^{2}$

Da es sich unbestreitbar um kollektive Gewaltanwendung handelte, zog der Vorfall rasch größere Kreise. Die Angelegenheit wurde vor dem Comes Orientis verhandelt, der sie wegen ihrer Brisanz dem Kaiser vorlegte. ${ }^{3}$ Daraufhin verfügte Theodosius I., der zu diesem Zeitpunkt in Italien weilte, in einem Edikt zweierlei: Zum einem sollten die kriminellen Mönche wegen Gewaltanwendung bestraft

1 Das Geschehen in seinen einzelnen Aspekten ist nahezu ausschließlich aus den interessegeleiteten Argumenten des Ambrosius in einem Brief an Theodosius I. sowie einem an seine Schwester zu rekonstruieren: Ambr. epist. 74 (40),6 (epist. extr. coll. 1a,6; zum zeitlichen Verhältnis von epist. 74 und epist. extr. coll 1a vgl. GROß-ALBENHAUSEN 1999, 100-101); 74 (40),8; 74 (40),13-14; 74 (40),16; epist. extr. coll. 1 (41),1. Zur Kallinikon-Affäre s. v.a. KolB 1980, 46-48; MCLYNN 1994, 298-309; GROß-ALBENHAUSEN 1999, 99-112 (letztere ist allerdings sehr wenig analytisch).

$2 \mathrm{Zu}$ den Auseinandersetzungen zwischen Christen und Juden in Antiocheia und der Übernahme der Grabstätte der makkabäischen Märtyrer durch die christliche Gemeinde HAHN 2004, 180189.

3 Ambr. epist. 74 (40),6. Mit der Bemerkung, daß noch kein Bericht des Bischofs von Kallinikon vorlag, als der Comes Orientis bereits seine Darstellung an den Kaiser gesandt hatte, betont Ambrosius die hohe Geschwindigkeit, mit der die Affäre auf die höchste Ebene transferiert wurde. Mit welcher Intention der Comes, wohl ein dezidierter Christ (zur Identifizierung FowDEN 1978, 67), hier gehandelt hat, wird allerdings nicht ganz klar. MCLYNN 1994, 299 n. 27 insinuiert einen möglichen Konflikt mit dem Präfekten von Konstantinopel, dem erklärten Nichtchristen Eutolmios Tatianos. Wahrscheinlich ist aber eher, daß der Comes nicht selbständig handeln wollte, weil ihm völlig klar war, was dann von ihm erwartet würde (s.u. n. 5). 
werden, zum anderen der Bischof - als Anstifter - die zerstörte Synagoge aus den Mitteln der christlichen Kirche wiederherstellen. ${ }^{4}$ Im Lichte der imperialen Ordnung erscheint dieses Urteil ebenso fair wie moderat, und insbesondere der Bischof von Kallinikon war dabei eigentlich gut weggekommen. Immerhin hatte er einen gewaltsamen Konflikt vom Zaun gebrochen, der die Ordnung zeitweilig außer Kraft setzte und die Bevölkerung seiner Stadt spaltete. ${ }^{5}$ Eine gewissermaßen rein zivilrechtliche Schadensersatzleistung konnte man bei dieser Sachlage eigentlich nur als Akt kaiserlicher clementia verstehen.

Das sah man allerdings im Kreis der Bischöfe des Reiches ganz anders. Dort sorgte das kaiserliche Edikt für beträchtliche Unruhe, und Ambrosius, dem Bischof von Mailand, fiel die Rolle zu, den Kaiser in die Schranken zu fordern. ${ }^{6} \mathrm{Er}$ sprach erst bei Theodosius vor und richtete dann eine briefliche Demarche an ihn, die an Deutlichkeit sogar sein späteres Ermahnungsschreiben wegen des Massakers in Thessalonike in den Schatten stellte. ${ }^{7}$ Als der Kaiser kurz darauf einen von Ambrosius in Aquileia geleiteten Gottesdienst besuchte, predigte der Bischof ostentativ gegen die Juden und warnte deren Parteigänger. ${ }^{8}$ Und noch in der Kirche, vor der Wandlung, kam es zwischen Gottesmann und Kaiser zur abschlieBenden Verhandlung der leidigen Affäre. Dabei war zwar Theodosius rasch bereit, dem Bischof von Kallinikon den Wiederaufbau der Synagoge zu erlassen; doch bestand er auf der Bestrafung der Mönche. Sie seien, so der Kaiser, nun einmal notorische Kriminelle. ${ }^{9}$ Ambrosius dagegen verlangte die vollständige Niederschlagung des Verfahrens gegen alle Beteiligte. Als Entscheidungshilfe verweigerte er dem Kaiser die Kommunion, bis dieser ihm ein förmliches und öffentliches Versprechen gegeben hatte. Theodosius' Nachgeben kommentierte Ambrosius in einem Brief an seine Schwester schließlich triumphierend mit der Bemerkung: „Und so geschah alles, wie ich es mir wünschte.“10

Aus der Perspektive des Imperiums war dieses Ende der Angelegenheit hingegen alles andere als befriedigend. Zwar hatte sich Theodosius am Ende gegen-

4 Ambr. epist. 74 (40),6; 74 (40),9-10; epist. extr. coll. 1 (41),1.

5 Für wie selbstverständlich Theodosius das Handeln der Administration in dieser Sache ansah, beweist seine explizite Ungehaltenheit darüber, daß der Comes Orientis, statt einfach die Schuldigen zur Rechenschaft zu ziehen, den Fall an den Kaiser weiterleitete (Ambr. epist. 74 (40),18).

6 MCLYNN 1994, 302.

7 Zum persönlichen Gespräch des Bischofs mit dem Kaiser: Ambr. epist. 74 (40),8-9. Für den Vergleich mit der Thessaloniki-Affäre s. Ambrosius' Brief an den Kaiser (epist. 11 (51)), der anders als in der causa Kallinikon - stärker von milder Trauer als von scharfem Vorwurf geprägt war. Auch daß sich der Bischof in der Einleitung von epist. 74 (40) veranlaßt sieht, die Parrhesia des Kirchenmannes gegenüber dem gerechten und rechtgläubigen Herrscher zu reklamieren, spricht für die exzeptionelle Deutlichkeit, mit der hier gesprochen werden sollte.

8 Ambr. epist. extr. coll. 1 (41),12-26 (an seine Schwester).

9 Ambr. epist. extr. coll. 1 (41),27.

10 Ambr. epist. extr. coll. 1 (41),28 (Übers. U. Gotter). 
über der Gemeinde von Aquileia als frommer Christ gezeigt, ${ }^{11}$ doch die öffentliche Revokation eines Edikts kann sein Prestige kaum gesteigert haben. Schlimmer noch war der Schaden für die Kalkulierbarkeit administrativen Handelns und den Glauben an die Geltung des Rechts. In einer Stadt des Reiches hatte es gewaltsame Ausschreitungen gegeben, man kannte die Anstifter und Akteure - und doch gingen alle straflos aus. Die Opfer wurden nicht einmal kompensiert, es gab keine symbolischen Akte der Reue, und sogar Maßnahmen zum Schutz vor ähnlichen Vorkommnissen blieben aus. Kurz: In der Affäre von Kallinikon hatte die Administration mit einem geheiligten Prinzip des römischen Staates gebrochen. Zwar basierte die römische Ordnung nicht auf der gleichmäßigen Durchsetzung uniformer Normen, ${ }^{12}$ wohl aber auf der strikten Ächtung von Gewalt als Mittel des Konflikts. ${ }^{13}$ Wo immer dergleichen auftrat, war nach traditioneller Auffassung die Grenze von Selbstbestimmung zu seditio, Aufruhr, überschritten. Und Aufruhr forderte im Grunde unweigerlich die Machtmittel des Imperiums heraus.

Vor diesem Hintergrund könnte man die Ereignisse von 388 n.Chr. durchaus als imperiale Katastrophe bezeichnen, wenn sie nicht nur die Spitze des Eisbergs gewesen wären. Denn was in Kallinikon und Aquileia geschah, war nicht das Ergebnis einer singulären persönlichen Konstellation, in der ein außergewöhnlicher Bischof einem bestimmten Kaiser heroisch die Sonderbehandlung seiner Klientel abtrotzte. Die Prominenz der Beteiligten und die Dramatik des Duells im Gotteshaus sorgten allenfalls dafür, daß wir über das Geschehen vergleichsweise gut informiert sind. ${ }^{14}$ Gewalt gegen jüdische Kultplätze und Gemeinden indessen war kein Einzelfall. Ebenfalls im Jahr 388, kurz vor dem Aufruhr in Kallinikon,

11 MCLYNN (1994, 308) spielt das Ergebnis der Konfrontation für den Kaiser systematisch und gegen die ältere Forschung herunter: „,The loser in this unhappy affair was Ambrose. Theodosius had been forced to concede clemency in a case he felt deserved exemplary punishment; but such concessions were an occupational hazard of the imperial office. As a compensation, moreover, he could enjoy the gratitude and admiration which he had no doubt inspired among the Christians of Milan. The bishop, however, had failed entirely to win the emperor's sympathy". Diese Sichtweise mag für die situative Kommunikationssituation der beiden Protagonisten nicht völlig unberechtigt sein - bereits KOLB 1980, 63-64 hat für die Beilegung der Kallinikon-Affäre und den Bußakt von Mailand den Zugewinn des Kaisers an Demutskapital hervorgehoben -, doch stellt sich die Sache für den zweiten Köper des Herrschers, seine imperiale persona, nicht ebenso einfach dar. Aus dieser Perspektive konnte der Verlust an Ordnung nicht durch die Demonstration von Frömmigkeit kompensiert werden.

12 Dies betonen die neueren Studien und synthetischen Darstellungen zum Imperium Romanum nahezu unisono: beispielhaft und explizit s. etwa MATTINGLY 1997; BARRETT 1997. Zu den primären und in der Tat gleichartigen Instrumenten zur Herstellung von akzeptierter Ordnung vgl. allerdings WHITTAKER 1997.

13 LENDON 1997, 2-7.

14 Dies hängt vor allem mit der Konservierung der entsprechenden Briefe im Corpus der Ambrosius-Werke zusammen. 
war eine Synagoge in Rom in Flammen aufgegangen $;{ }^{15}$ weitere Angriffe gab es in den letzten Dezennien des 4. und zu Beginn des 5. Jahrhunderts. ${ }^{16}$

Dennoch träfe es nicht den Kern, diese Ereignisse unter dem Lemma eines wachsenden Antijudaismus oder Antisemitismus einzuordnen. ${ }^{17}$ Das legt eigentlich schon das Geschehen in Kallinikon selbst nahe. Denn die Juden waren keineswegs die einzigen Leidtragenden von Gewalt gegen Kultstätten. Seit der Mitte des 4. Jahrhunderts kam es immer wieder zu Übergriffen auf pagane Heiligtümer, ${ }^{18}$ ähnliches gilt für kleinere religiöse Gruppierungen wie Samaritaner, Valentinianer und Manichäer. ${ }^{19}$ Und selbst christliche Kirchen der falschen Ausrichtung oder des falschen Bischofs blieben von gewaltsamen Übernahmen und plötzlichem Brand nicht verschont. ${ }^{20} \mathrm{Da}$ es dabei unweigerlich auch zu Personenschäden kam, bedarf nicht der Erwähnung. Die dezentrale und illegale ${ }^{21}$ Gewaltausübung gegen Kultorte, Kultgruppen und ihre Riten war also längst zu einem Phänomen geworden, das das politische Klima im Reich umfassend prägte und in den folgenden Jahrzehnten noch eine bedeutende Karriere vor sich hatte. ${ }^{22}$ Unter diesen Umständen demonstriert die Kallinikon-Affäre lediglich besonders eindrucksvoll die Ohnmacht des spätantiken Staates, die traditionellen Normen des Imperiums durchzusetzen. Daß den Beteiligten dieses Problem auch durchaus erkennbar war, zeigt die Reaktion des Theodosius, der sich die Bestrafung der Schuldigen zunächst nicht vollständig abhandeln lassen wollte.

Im folgenden will ich einerseits nach Gründen für diese Ohnmacht fragen und andererseits präziser zu umreißen versuchen, was die Gewaltanwendung zwischen religiösen Gruppen für die politische Kultur des Reiches bedeutete. Dazu soll in einem ersten Schritt rekapituliert werden, über welche Mechanismen zur Unterdrückung von Gewalt der kaiserzeitliche Staat verfügte und warum diese so erfolgreich waren. Sodann wird - gewissermaßen als Gegenprobe - mit einem

15 Ambr. epist. 74 (40),23; s. NoETHLichs 1971, 184-185. Für die Umwandlung der Synagoge von Apameia in eine christliche Kirche und die antijüdischen Kontexte dieses Vorgehens vgl. BRENK 1991.

16 Bekannt sind insbesondere die Aktionen des Mönches Barsauma in Syrien, das, wie BRENK 1991, v.a. 14-19; 22-24 gezeigt hat, ein regionaler Schwerpunkt für die Zerstörung bzw. Umwandlung von Synagogen in Kirchen war; s.a. STEMBERGER 1987, 247-249; MiTCHELL 1999, 234-238

17 So etwa SAFRAI 1994, 435-436.

18 Dazu jetzt HAHN - EMMEL - GOTTER 2008. Eine breite Fallsammlung findet sich bereits bei TROMBLEY 1993/1994; s.a. HAHN 2004, v.a. 191-270.

19 NOETHLICHS 1986, 1180-1182; STEMBERGER 1987, 182.

20 GADDIS 2005, 79-88; HAHN 2004, 48-77; ISELE 2008.

21 Kaiser Maximus ächtete in einem Edikt das gewaltsame Vorgehen gegen jüdische Kultorte und drohte dafür wohl schwere Strafen an (vgl. NOETHLICHS 1971, 182-188). Zum Rechtsstatus der Juden im Reich s. RABELLO 1980, der seinerseits auf dem umfassenden Werk JUSTERS 1914 beruht; zum Schutz der jüdischen Kultorte im 4. und 5. Jahrhundert JUSTER 1914, Bd. 1, 460472; LINDER 1987, 85-86; BRENK 1991, 12-14.

22 Vgl. TROMBLEY 1993/1994; zur Rolle der Bischöfe dabei FowDEN 1978. 
Blick auf den ersten jüdischen Aufstand gezeigt werden, unter welchen Bedingen diese imperialen Mechanismen nicht funktionierten, bevor ich in einem dritten Abschnitt auf Kallinikon, die Dysfunktionalität der traditionellen imperialen Normen unter christlichen Auspizien und die Konsequenzen des Prozesses zurückkomme.

\section{Kaiserzeitliches Gewaltmanagement}

Womöglich war das Imperium Romanum gerade deshalb so effizient, weil es nicht versuchte, Konflikte unter den Beherrschten wirklich zu lösen bzw. dauerhaft auszuräumen. ${ }^{23}$ Das nämlich hätte positive sachliche Zielvorgaben verlangt, eine materielle Definition von Ordnung und eine flächendeckende Festlegung von Normen, die angesichts der enormen kulturellen und politischen Differenzen innerhalb des Reiches niemals einen stabilen Konsens generiert hätten. Statt dessen begnügte man sich mit der Setzung von institutionellen Rahmenbedingungen, innerhalb derer die subsidiären, regionalen politischen Organisationsformen zumindest partiell intakt bleiben konnten. Als ordnungspolitisches Gegengewicht forderte diese administrative Minimallösung aus römischer Perspektive allerdings zumindest, die Austragung von Konflikten zu reglementieren bzw. zu entschärfen. Konkret bedeutete das schlichtweg die Ächtung von kollektiver und individueller Gewaltanwendung auf lokaler Ebene. ${ }^{24}$ Bei aller Autonomie der subsidiären Einheiten machte die Zentrale unmißverständlich klar, daß der Einsatz von Gewalt den Eingriff der Institutionen des Reiches provozierte. Das galt natürlich in erster Linie für die Zusammenrottung von Gruppen, die die Funktion von lokalen Gemeinschaften unmittelbar bedrohten oder sich gar gegen die römische Ordnung auf Provinzebene richteten. Bezeichnend ist aber, daß auch die kriminelle Gewaltanwendung durch den Einzelnen, wenn sie eine Kapitalstrafe erwarten ließ, die Reaktion der Zentralmacht regelhaft provozierte. In diesen Fällen wurde die Rechtsprechung und Ahndung des Verbrechens der lokalen Einheit entzogen und den Institutionen der Reichsverwaltung überantwortet: ${ }^{25}$ Auch die äußerste legale Gewalt war damit zumindest idealerweise monopolisiert.

23 Vgl. treffend WolfF 1999, 60: „Es scheint mir ein (...) Geheimnis des Erfolges römischer Reichspolitik wie auch der Reichsadministration gewesen zu sein, daß sie beide nicht auf positive Utopien festgelegt waren, sondern solche Vorgaben nur sehr allgemein und vor allem negativ formulierten."

24 DAHLHEIM 2003, 100-114; 237-239; JACQUES - SCHEID 1998, 192-198; ECK 1999; MITCHELL 1999, 25-29; BLEICKEN 1978, Bd. 1, 130-132.

25 NÖRR 1966, 32-33 (zu Regel und Ausnahme); GARNSEY 1968, 51-55; STAHL 1978, 90-99; JACQUES - SCHEID 1998, 197; JÖRDENS 1999, 154; 163-164 (für Ägypten); HORSTKOTTE 1999, 309-310; 313; zu den Ausnahmen: 307. NÖRR 1999, 261-262 betont das Mißtrauen der Griechen gegenüber ihrer eigenen „Gerechtigkeit“. 
Diese Regelung war hochgradig funktional und traf präzise den Nerv des Problems. Vor allem die östlichen griechischen bzw. hellenisierten Polisgesellschaften waren hochkompetitive Einheiten, die zu existentiellen Konflikten mit latenter Bürgerkriegstendenz neigten. Im Zentrum dieser Auseinandersetzungen standen Cliquen, die erhebliche Teile der Bürgerschaft über meist binäre Optionen mobilisieren konnten. ${ }^{26}$ Klassisch in diesem Sinne war die Antithese von Demokraten und Oligarchen, von ,Armen' und ,Reichen', später von Romfreunden und Romfeinden. Auf den sachlichen Gehalt dieser Auseinandersetzungen kam es dabei allerdings nicht in erster Linie an: Im Prinzip taugte jede bedeutsame Entscheidung für die Organisation von lokalen Konflikten. ${ }^{27}$ Und einerlei nach welchen ideologischen Positionen sie sich entfalteten - Mitglieder der Führungsschicht waren auf beiden Seiten tonangebend, selbst im Rahmen der angeblich sozialen Auseinandersetzungen.

Für die Schärfe ihrer Austragung indessen war die Gewaltfrage von entscheidender Bedeutung. Die enorme zentrifugale Wirkung von Gewaltakten im griechischen Kontext hängt wohl vor allem damit zusammen, daß Gewalt dort außerordentlich zeichenhaft und aufs engste mit dem politischen Zentralbegriff der Macht verknüpft war. So erschöpfte sich Gewaltanwendung aus der Perspektive der Beteiligten nicht im physischen Akt und seinen unmittelbaren Konsequenzen, sondern verkörperte darüber hinaus gleichzeitig einen Anspruch von größerer Reichweite. Wer es sich leisten konnte, einen oder mehrere andere zu schlagen, $\mathrm{zu}$ verletzen oder zu töten, reklamierte damit gleichzeitig Vorrang und Übermacht. ${ }^{28}$ Hinter dieser Wahrnehmung lauerte die Gefahr, daß auch die sehr partikulare, gewissermaßen private Gewaltanwendung rasch ins Kollektive abglitt und schließlich in äußerster Eskalation endete. Im griechischen Osten schuf, oder besser: zementierte Gewalt schnell Parteiungen.9 Die grundsätzliche Konfliktkonstellation in den Poleis überlebte auch die politischen Veränderungen während des Hellenismus und der römischen Kaiserzeit. ${ }^{30} \mathrm{Da}$ man sich auf römischer Seite der Latenz eines zentrifugalen Parteienwesens durchaus bewußt war, zeigt etwa die strikte Weigerung Trajans, ein pragmatisch gebotenes FeuerwehrCollegium in Nikaia zuzulassen: „Du bist auf den Gedanken gekommen, man könne nach dem Vorbild mehrerer anderer Städte in Nikomedia eine Handwerkergilde bilden. Aber vergessen wir doch nicht, daß deine Provinz und vornehmlich

$26 \mathrm{Zu}$ den Prinzipien des inneren Krieges in Griechenland vgl. v.a. GEHRKE 1985, 205-353; zur Bipolarität der Konflikte GEHRKE 1985, 245-249; s.a. CARTLEDGE 1993, 111-114.

27 DEININGER 1971, 12-20; GEHRKE 1985, 309-353.

28 S. TRAMPEDACH 2006, mit dem Hinweis auf die latente Attraktivität der Tyrannis.

29 Zum entsprechenden Muster, das sich um das Motiv der Rache organisiert, GEHRKE 1987, 130$136 ; 144-145$.

30 Zur Existenz von Auseinandersetzungen in den griechischen Städten des Hellenismus und der Kaiserzeit: SARTRE/SARTRE 2000; BENCIVENNI 2003; DösSEL 2003. Eine systematische Analyse der Konfliktkonstellationen und Stasisphänomene nach dem 4. Jhdt. v. Chr. bleibt jedoch ein unbedingtes Desiderat der Forschung. 
ihre Gemeinden unter derartigen Organisationen zu leiden gehabt haben. Einerlei, aus welchem Grunde wir sie zulassen, und welchen Namen wir den Leuten geben, die für einen bestimmten Zweck organisiert werden, es werden immer, und zwar in ganz kurzer Zeit, Hetärien daraus werden“. 31

Unter diesen Umständen war es ein weitsichtiger Akt der römischen Administration, die Regelung gewaltsamer Konflikte und die Ahndung von Gewalttaten dem lokalen Biotop vollständig zu entziehen. Denn erfahrungsgemäß konnten selbst ordentliche Gerichtsurteile auf der Ebene städtischer Gemeinschaften nur schwer das akzeptanzvernichtende Stigma der Parteilichkeit vermeiden. ${ }^{32}$ Mit dem Prinzip der systematischen Entfernung des Urteils über Gewalttaten von ihrem Schauplatz - dabei galt die Faustregel: je bedeutender die Beteiligten, desto höher die richtende Instanz ${ }^{33}$ - vermochte Rom dagegen eine glaubhafte Position der Neutralität einzunehmen. Diese Neutralität erstreckte sich auch vor allem auf die Begründung für Gewaltanwendung. Gewalt selbst war der entscheidende Tatbestand, der geahndet wurde, nicht das Motiv hinter der Gewalt. Aus der Perspektive der Reichsverwaltung waren der Grund und die Begründung für Gewaltanwendung nebensächlich.

Berücksichtigt man die besondere kulturelle Disposition der östlichen Gemeinschaften, erscheinen Distanz und Neutralität als die zentralen Instrumente der pax Romana. Der Gewalt, des wichtigsten Katalysators von Bürgerkriegen beraubt, waren die östlichen Eliten zur zumindest zähneknirschenden Kooperation gezwungen.

Was aber garantierte die Akzeptanz eines römischen Diktats, das immerhin tief in kulturelle Verhaltensweisen eingriff und das politische Klima im römischen Osten grundlegend verwandelte? Kurioserweise war hierfür wohl gerade derjenige Zug griechischer politischer Wahrnehmung entscheidend, der auf lokaler Basis eine zentrifugale Wirkung entfaltete: die Obsession für das Argument der Macht. ${ }^{34}$ Entscheidend war dabei nicht unbedingt die tatsächliche Macht des römischen Imperiums, also gewissermaßen die Pistole an der Schläfe der Akteure, sondern die Wahrnehmung Roms als unüberwindlich. Dieses Dogma hatte sich in einem schmerzhaften und von brennenden Städten gesäumten Lernprozeß tief in das griechische Weltverständnis eingegraben. ${ }^{35}$ Gewohnt, die Welt als ein

31 Plin. epist. 10,34,1: tibi quidem secundum exempla complurium in mentem venit posse collegium fabrorum apud Nicomedenses constitui. sed meminerimus provinciam istam et praecipue eas civitates eius modi factionibus esse vexatas. quodcumque nomen ex quacumque causa dederimus iis, qui in idem contracti fuerint, hetaeriae eaeque brevi fient (Übers. $\mathrm{H}$. Kasten). Zum römischen Mißtrauen gegen das Vereinswesen zuletzt BENDLIN 2005.

32 Zu Losverfahren und Bestechungsverdacht s. HANSEN 1991, 204-205; GEHRKE 1985, 208-210; DÖSSEL 2003, 277.

33 JACQUES - SCHEID 1998, 197; STAHL 1978, 148-158; HORSTKOTTE 1999, 305-306. Zur sozialen Ausdifferenzierung des Rechtsapparats vgl. RILINGER 1988.

34 Hierzu jetzt GOTTER 2008, 183-199.

35 DEININGER 1971, 191-261; 269-270; GEHRKE 2003, 122-131; 227-230. 
System von Machtbeziehungen zu analysieren, erschien letzten Endes die Unterordnung unter fremde Prinzipien zwar nicht gerade als begrüßenswert, doch immerhin als plausibel, wenn sich an der Übermacht des anderen nun einmal nichts ändern ließ ${ }^{36}$

Diese Kapitulation vor dem Unvermeidbaren bringt Plutarch in seinen Praecepta gerendae rei publicae auf den Punkt, wenn er im 2. Jahrhundert n. Chr. den zeitgenössischen griechischen Polisaristokraten folgenden politischen Imperativ verordnet:

„Und wenn du irgendein Amt übernimmst, mußt du dir nicht nur die Maximen ins Gedächtnis rufen, die Perikles memoriert hat, als er den Mantel eines Generals anlegte: ,Denk daran, Perikles, du beherrschst freie Männer, Griechen beherrschst du, athenische Bürger', sondern du mußt dir auch sagen: ,Du herrschst über eine Stadt als ein Beherrschter, kontrolliert von Prokonsuln, den Agenten des Kaisers. (...) Du solltest also deinen Mantel aufmerksamer arrangieren und vom Amt des Generals weg auf die Redebühne schauen und nicht deine Krone so hochschätzen und ihr vertrauen, wo du doch den Stiefel des römischen Soldaten direkt über deinem Kopf siehst. Vielmehr sollst du die Schauspieler nachahmen, die in ihre Rolle das eigene Pathos, den eigenen Charakter und die eigene Würde legen, doch auf den Souffleur hören und in Reimen und Versmaß nicht die Freiheit überschreiten, die ihnen von den über sie Herrschenden eingeräumt wird. “37

Selbst wenn also die Ansprüche des Imperiums mit den eigenen kollidieren sollten oder die Normen des Imperiums im Grunde nicht akzeptabel sein mochten, ${ }^{38}$ galt doch der Grundsatz, daß man gegen die Übermacht des Diktats nichts Erfolgversprechendes ausrichten konnte. Auf diese Weise war die Aura der Unbesiegbarkeit des Imperiums der rocher de bronze, auf dem die Akzeptanz der römischen Ordnung im griechischen Osten ruhte.

36 Der Topos von der Unbesiegbarkeit Roms beherrscht den griechischen intellektuellen Diskurs über Rom seit Polybios: DAHLHEIM 2003, 111f; DEININGER 1971, 270-273.

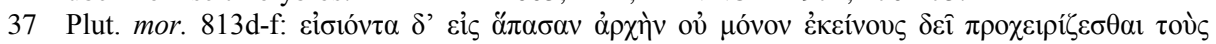

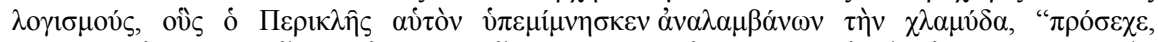

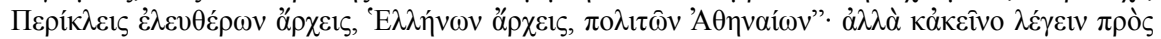

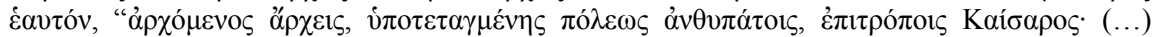

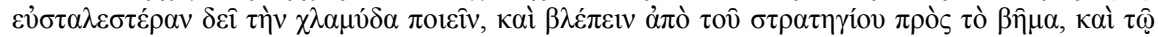

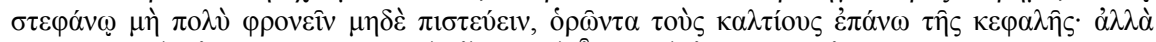

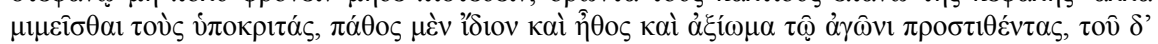

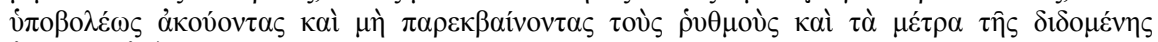

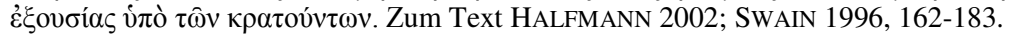

38 So beklagt Plutarch (798C-806B), daß eine heroische aristokratische Profilbildung (die Blutvergießen zwingend beinhaltete) durch das römische Diktat unmöglich gemacht worden sei. Statt dessen seien nur noch Talente gefragt, die auf friedliche Konkurrenz abzielten. Aelius Aristides formuliert übrigens denselben Tatbestand, bewertet ihn allerdings ausschließlich positiv (Ael. 68-69; 96-99). Gegenüber dieser Friedhofsruhe (824C-D) scheint in Plutarchs Text die Sehnsucht nach existenziellen Konflikten auf (s. HALFMANN 2002, 87-92). SwaIN (1996, 171) faßt die Haltung des Mannes aus Chaironeia folgendermaßen zusammen: „His own advice is to stay at home and, in brief, not to integrate“. 


\section{Gegenprobe: Die Grenzen der römischen Ordnung}

Die Propagierung des übermächtigen Imperiums war nahezu überall erfolgreich. Es gab allerdings eine Ausnahme, und diese ist heuristisch außerordentlich wertvoll. Denn sie bestätigt einerseits den Befund und zeigt andererseits präzise an, wo die Grenzen des römischen Herrschaftssystems lagen. War die Akzeptanz der römischen Ordnung im östlichen Mittelmeerraum insgesamt stabil, galt dies nicht für Judäa im ersten und zweiten Jahrhundert $\mathrm{n}$. $\mathrm{Chr}^{39}$ Eine Integration in das Imperium gelang dort nur sehr rudimentär: So rückten anders als in den anderen östlichen Provinzen Angehörige der jüdischen Oberschicht nicht in die Funktionseliten des Reiches auf. Zwar gab es auch jüdische Angehörige des ordo equester, des reichsrömischen Ritterstandes, doch blieb dies ein Phänomen des sozialen Status. Denn die Übernahme von Ämtern, die eigentlich mit dem Ritterrang verbunden war, hätte die Ausübung religiöser Kulte bedeutet, die einem Juden verboten war. ${ }^{40}$ Ein Angehöriger der jüdischen Führungsschicht hatte also regelmäßig zwischen einer administrativen Karriere und der Zugehörigkeit zu seiner traditionellen Religionsgemeinschaft zu wählen. ${ }^{41}$ Damit war für den jüdischen Fall die Einbindung regionaler Oberschichten in den imperialen Apparat ausgeschlossen, und der römischen Herrschaft in Judäa fehlte ein wesentlicher Transmissionsriemen. Daß dies insbesondere im Rahmen eines Ordnungssystems problematisch war, das seit Jahrhunderten darauf fixiert war, regionale Integration über die Einbindung indigener Führungseliten herbeizuführen, liegt auf der Hand. Die funktionale Abstinenz der jüdischen Oberschicht machte sich in den Eskalationen im Judäa des ersten nachchristlichen Jahrhunderts außerordentlich negativ bemerkbar. Während etwa die übrigen Bewohner Palästinas und Syriens Vertreter

39 Zu den Konflikten zwischen Juden und Römern in Judäa: BALTRUSCH 2002, 129-147; HENGEL 1976; HENGEL 1999, 149-152; WILKER 2007, v.a. 377-470. Dagegen weist GOODMAN 1991 auf die seiner Meinung nach geringe Evidenz für eine allgemeine Ablehnung der römischen Herrschaft in Judäa hin. Entscheidend ist allerdings, daß für die zelotische Position, auch wenn sie zahlenmäßig in der Minderheit gewesen sein mag, mit der Schrift eine mächtige Option eines Anti-Diskurses zur Verfügung stand, der, wenn es sich anbot, im jüdischen Kontext wirksamer aktualisiert werden konnte als andernorts. In seinem Bemühen, die Besonderheit der judäischen Verhältnisse zu negieren, verzerrt Goodman zunächst die Evidenz, indem er Grenzgebiete mit Regionen im Herzen des Imperiums vermengt (225-230). Seine Position, daß das Reich insgesamt lediglich durch uniforme konstante Furcht zusammengehalten worden wäre (236-237), ist ebenso holzschnittartig wie soziologiefern. Geradezu absurd ist die These, daß auch bei den anderen Aufständen im Reich die religiöse Mobilisierung relevant gewesen sei (237-238). Hier überschätzt Goodman die Möglichkeit des Polytheismus bei weitem, Konflikte über Kultgruppen zu generieren.

40 JUSTER 1914, Bd. 2, 243-244; 248-249.

41 Ein bezeichnendes Beispiel dafür ist Tiberius Alexander, der Befehlshaber der ägyptischen Legionen, die 69 n. Chr. Vespasian zum Kaiser ausriefen: Von jüdischer Herkunft, opferte er seine Zugehörigkeit zum aktiven Judentum einer steilen Militärkarriere (s. JUSTER 1914, Bd 2; 249 n. 3 und 4$)$. 
ihres Ethnos im administrativen Apparat hatten - in der Regel Figuren, die meist keine Sympathien für die Juden hegten ${ }^{42}-$, gab es auf jüdischer Seite dazu kein Gegengewicht.

Die Integration Judäas in das Imperium war allerdings nicht nur wegen mangelnder funktionaler Repräsentation seiner Oberschicht fragil. Auch die prinzipielle Beugung unter die römische Macht war nicht dauerhaft konsensfähig. Daß das ordnungsvermittelnde Standardargument, die Unbesiegbarkeit des Imperiums, in Judäa nicht zog, war für Flavius Josephus, den jüdischen Historiker und Augenzeugen des großen Aufstandes, jedenfalls bemerkenswert genug, um darin eine wesentliche Erklärung für die Eskalation des Konflikts mit Rom zu sehen. In seinem Werk über den ,Jüdischen Krieg' macht er aus dem Thema eine Schlüsselszene für den Weg der Juden in die militärische Katastrophe. Der König des nördlichen Palästina und Aufseher über den Jerusalemer Tempel, Agrippa II., versucht seine Landsleute durch eine Rede vom Waffengang abzuhalten und zur Umkehr zu bewegen. Bezeichnenderweise läßt ihn Josephus nicht in erster Linie über Recht und Unrecht diskutieren, sondern über (macht)politische Opportunitäten:

„Ihr, die ihr schon das Gehorchen als Erbschaft empfangen habt, und in euren Hilfsmitteln jenen, die als erste diesen Gehorsam auf sich nahmen, so weit unterlegen seid, ihr wollt der römischen Gesamtstreitmacht Widerstand leisten? Schaut die Athener an, die einst um der Freiheit der Griechen willen ihre Stadt sogar dem Feuer preisgaben. (...) Sie, die bei der kleinen Insel Salamis das so mächtige Asien zerbrachen, dienen jetzt den Römern, und über die Stadt, die einst Herrin Griechenlands war, verfügen jetzt die Weisungen aus Italien. (...) Tausende von anderen Völkern, erfüllt von tiefem Verlangen nach Freiheit, haben sich gebeugt; ihr allein haltet es für unwürdig, denen zu dienen, denen sich die Welt untergeordnet hat. Wie sieht das Heer und die Bewaffnung aus, auf die ihr vertraut? Wo ist bei euch die Flotte, die die römischen Meere in Besitz nehmen soll? Wo ist ein ausreichender Kriegsschatz für eure Unternehmungen? (...) Habt ihr denn gar keine Vorstellung von der Macht Roms und eurer eigenen Schwäche? Sind unsere Heere nicht oft auch schon den Nachbarvölkern unterlegen, während die Macht Roms auf der gesamten Erde unbesiegt blieb? Sie suchten sogar die Grenzen des Erdkreises noch zu überschreiten. (...) Wie steht es nun? Seid ihre reicher als die Gallier? Stärker als die Germanen? Klüger als die Griechen? Seid ihr zahlreicher als alle Völker der Erde? Was für ein Selbstvertrauen gibt euch den Mut, euch gegen die Römer zu erheben?““43

42 Vgl. SCHAEFER 1997, 170-179 über die antijüdischen Kontexte in Syrien und Palästina.

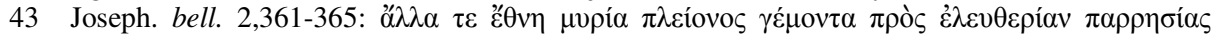

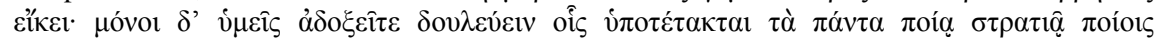

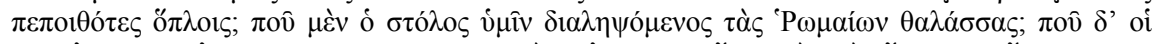

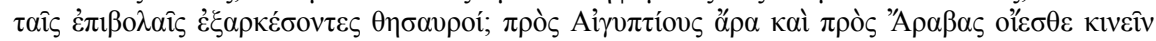

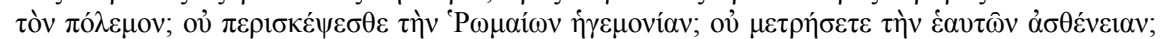

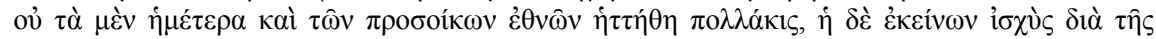

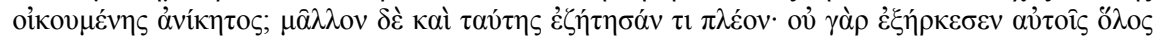


Der prophetische Charakter von Agrippas Rede spricht unbestreitbar für ihre $e x-$ eventu-Komposition. ${ }^{44}$ Doch gerade wenn es sich hierbei um ein Konstrukt des Josephus handelt, ${ }^{45}$ bestätigt dies nur die enorme Präsenz der Kategorie „Macht“ im griechischen Kontext. Josephus inszeniert in dieser Passage das stärkste Argument für Rom in der östlichen Welt. Eine explizite Erklärung dafür, warum der König damit bei der Jerusalemer Öffentlichkeit keinen dauerhaften Erfolg hatte, gibt der Historiker indessen nicht. Er deutet lediglich an, daß es die Religion gewesen sei, die die Akteure gegen das immunisierte, was Agrippa als vernünftig beschrieb. ${ }^{46}$ Und in der Tat kann angesichts des jüdischen Gottesverständnisses ein rein säkulares Machtargument aus mindestens zwei Gründen keine Valenz beanspruchen. Einerseits sorgte die transzendent ausgerichtete Normenbegründung im jüdischen Kontext ${ }^{47}$ dafür, daß innerweltlicher Opportunismus nicht der alleinige Maßstab für Entscheidungen im Konfliktfall sein konnte. Auf der anderen Seite ließ sich aus der unbestrittenen Annahme der Macht Jahwes leicht eine verführerische politische Option schmieden, getreu dem Motto: ,Wenn Gott für uns ist, wer kann dann gegen uns sein $?^{48}$ Zumindest die Radikalen, die Zeloten, haben mit der Verheißung göttlicher Unterstützung für die gerechte Sache Israels immer wieder Anhänger mobilisieren können. ${ }^{49}$ Für den Erfolg dieser Position spielte m.E. die Rezeption des siegreichen Aufstandes der Makkabäer gegen den Seleukidenkönig Antiochos IV. eine unheilvolle Rolle. ${ }^{50}$ In der jüdischen Tradition ist daran die Konfrontation zwischen illegitimer gottloser Macht und rechtgläubiger

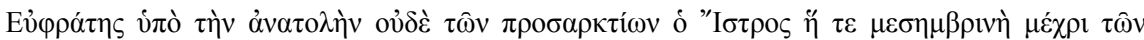

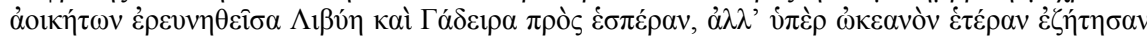

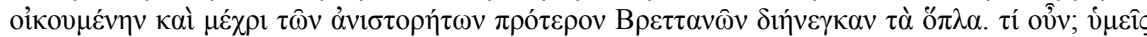

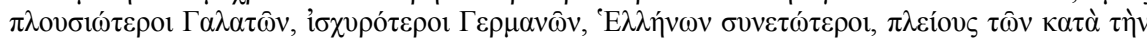

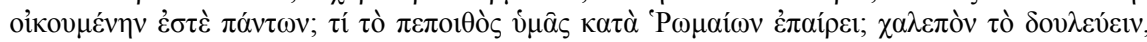

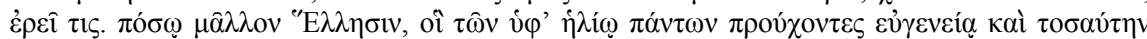

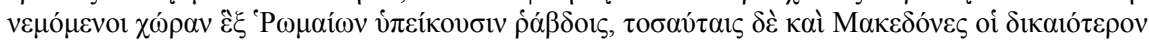

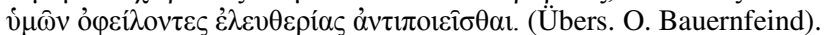

44 So wird in der Rede der Untergang des Judentums und die Zerstörung des Tempels imaginiert (Joseph. bell. 2,394-400).

45 Zu Form, Kontext und Bedeutung der Rede für Josephus' Werk: RAJAK 1991; MASON 2008, MASON 2008a, Abschnitt IV.

46 Nachdem Agrippa gemäß Josephus' Schilderung vorerst die Krise abzwenden konnte, gelang ihm dies wenig später nicht mehr. Die von der Kriegspartei formulierten Argumente läßt der Historiker allerdings nur in den Gegenargumenten der Friedenswilligen aufscheinen (Joseph. bell. 3, 408-417).

47 So markant HeNGEL 1976, v.a. 151-172; 229-234; TRAMPEDACH 2007, 61-65.

48 Diese Formulierung von Röm 8,31 basiert auf Jes 50,7-9 und 1Sam 14,6. Das Motiv findet sich einflußreich natürlich bereits im Buch Exodus.

49 TRAMPEDACH 2007, 40-48.

50 BRINGMANN 1983, 146-148; HENGEL 1976, bes. 38-48; s.a. MASON 2008a, Abschnitt IV mit dem Verweis darauf, daß Agrippa in seiner Rede bei Josephus das Vorbild des siegreichen Aufstandes bewußt klein redet, um das Unruhepotential in Palästina zu vermindern. 
nomineller Schwäche idealtypisch durchgespielt worden. Ich zitiere nur eine der vielen einschlägigen Stellen aus dem 1. Makkabäerbuch:

„Judas [Makkabäus] zog [dem syrischen Feldherrn Seron] mit ganz wenigen Männern entgegen. ${ }^{17}$ Als diese das Heer sahen, das gegen sie ausgerückt war, sagten sie zu Judas: Wie können wir mit so wenigen Leuten gegen eine solche Übermacht kämpfen? (...) ${ }^{18}$ Judas antwortete: Es kann leicht sein, daß viele wenigen in die Hände fallen; für den Himmel macht es keinen Unterschied, ob er durch viele oder wenige Rettung bringt. ${ }^{19}$ Denn der Sieg im Kampf liegt nicht in der Größe des Heeres, sondern in der Kraft, die vom Himmel kommt. ${ }^{20}$ Diese Leute da ziehen voll Hochmut und Bosheit (hybris kai anomia) gegen uns in den Kampf, um uns mit unseren Frauen und Kindern auszurotten und unsere Habe zu plündern. ${ }^{21}$ Wir aber kämpfen für unser < rechtes> Leben und für unsere Gesetze (psychai kai nomoi). ${ }^{22}$ Der Himmel wird sie vor unseren Augen vernichtend schlagen. Darum habt keine Angst vor ihnen! ${ }^{23}$ Kaum hatte er das gesagt, da stürzte er sich überraschend auf die Feinde, und Seron und sein Heer wurden vor seinen Augen aufgerieben." ${ }^{\text {(51 }}$

Die Botschaft, die diese Version des Kampfes der Makkabäer gegen die nichtjüdischen, verbrecherischen und sündigen Gegner Israels transportiert, ist unübersehbar: Wird für eine gerechte Sache gestritten, spielt die Macht des Feindes keine Rolle. Gott zählt mehr als die fehlenden Bataillone. Wenn dergleichen als Muster historischer Interpretation und als Maxime politischen Handelns gesellschaftsfähig wurde, war die Evidenz der römischen Ordnung strukturell aufgehoben.

\section{Diskurse der Gewalt}

Aus der imperialen Perspektive bildete das Christentum gewissermaßen die Potenzierung des jüdischen Problems. Immerhin waren die Juden eine präzis abgrenzbare Gruppe, die im Extremfall - dem kollektiven Aufruhr - das Objekt einer militärischen Operation sein konnte. Dagegen zeigte sich rasch, daß die

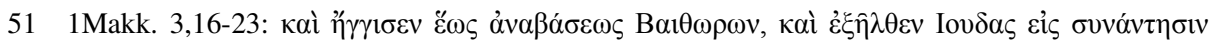

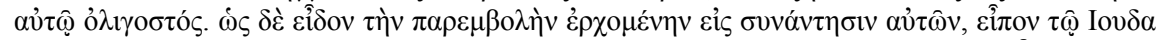

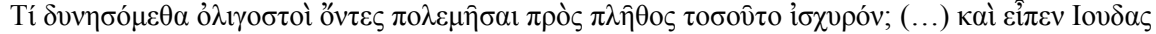

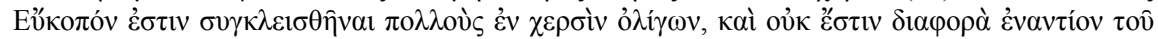

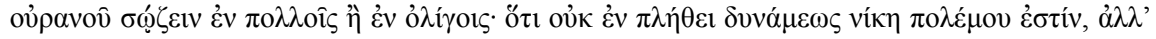

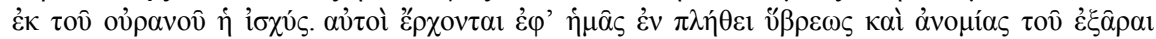

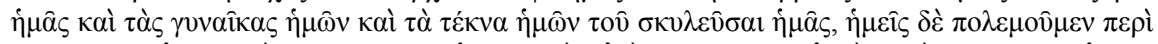

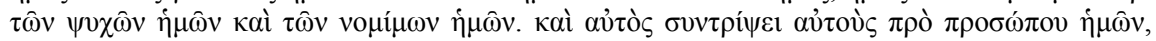

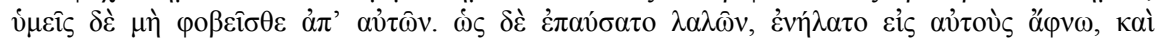

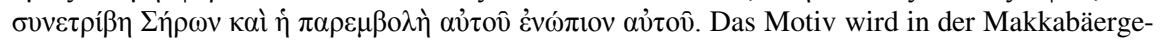
schichte häufig wiederholt, etwa 1 Makk 4,6-11; 4,28; 4,34. 
Gewaltfrage unter christlichen Auspizien eine unlösbare Aufgabe für das Imperium darstellte.

Die konkreten Probleme, die die Christianisierung für die traditionelle Ordnung aufwarf, beleuchtet der Ambrosius-Brief an Theodosius grell und exemplarisch: Sein Bündel von Argumenten für die Niederschlagung der KallinikonAffäre ist geradezu eine Blaupause der verfügbaren und eingesetzten Rechtfertigungen von Gewalt, die die klassischen Instrumente des Reiches aushöhlten und unterliefen. ${ }^{52}$ Entscheidend ist bereits der Parameter, den Ambrosius an die Auseinandersetzungen von 388 anlegt: Statt den Vorfall in Kallinikon als ordnungspolitische Aufgabe zu begreifen, die in der Tat ein Monopol des Kaisers wäre, etikettiert er sie als Glaubensfrage, die die exklusive Kompetenz des säkularen Herrschers überschreitet. ${ }^{53}$ In Ambrosius' Version der Ereignisse stehen sich nicht Gewaltopfer und Gewalttäter gegenüber, sondern die rechtgläubige Kirche Christi und die Synagoge, die er als „Heimstatt der Unfrommen und der Unvernunft, die Gott selbst verdammt habe“, apostrophiert. ${ }^{54}$ Die Wiederherstellung der zerstörten Synagoge durch den Bischof und aus den Mitteln der Kirche sei schon deshalb völlig undenkbar, weil dies den Triumph des gottlosen Konkurrenten und religiösen Gegners bedeutet hätte. ${ }^{55}$ Aus einer solchen, transzendent armierten Freund-Feind-Perspektive ist die Rechtsfrage irrelevant und die Ordnung des Reiches zweitrangig. Daß sich Ambrosius damit gegen die traditionellen imperialen Prinzipien wendet, ist ihm völlig bewußt, ja er formuliert das Problem ebenso offen wie brutal:

„Aber vielleicht ist es die Ordnung (disciplina), um die es dir zu tun ist, Kaiser. Doch was ist wichtiger? Die Durchsetzung der Ordnung oder die Sache der Religion? Es ist da allemal notwendig, dem Glauben nachzugeben".56

Ambrosius' Argument kulminiert in der Proklamation, er selbst würde bei Bedarf ebenso handeln wie der Bischof von Kallinikon und die Häuser der Gottlosen an-

52 Zur religiösen Rechtfertigung von Gewalt jetzt grundsätzlich GADDIS 2005, passim, bes. 21-22.

53 Ambr. epist. 74 (40),3 (epist. extr. coll. 1a,3): non ergo importunus indebitis me intersero, alienis ingero: sed debitis obtempero, mandatis Dei nostri obedio; s.a. epist. 40,5. Aus dieser Perspektive konsequent ist Ambrosius' Vorschlag, die Angelegenheit mit einem consilium von Bischöfen zu diskutieren: certe si mihi parum fidei defertur, jube adesse quos putaveris episcopos: tractetur, Imperator, quid salva fide agi debeat. Si de causis pecuniariis comites tuos consulis, quanto magis in causa religionis sacerdotes Domini aequum est consulas (epist.74 (40),27 (epist. extr. coll. 1a, 27).

54 Ambr. epist. 74 (40),9-15; Zitat 74 (40),14: non est ergo causa tantae commotioni idonea; ut propter aedificii exustionem in populum tam severe vindicetur: multo autem minus quia synagoga incensa est, perfidiae locus, impietatis domus, amentiae receptaculum, quod Deus damnavit ipse (Übers. U. Gotter).

55 Ambr. epist. 74 (40),10: (...) hunc titulum Iudaei in fronte synagogae suae scribent: templum impietatis factum de manubiis christianorum.

56 Ambr. epist. 74 (40),11: sed disciplinae te ratio, imperator, movet. Quid igitur est amplius? disciplinae species, an causa religionis? cedat oportet censura devotioni. 
zünden. ${ }^{57}$ Gottes Gebot stehe über den Gesetzen des Kaisers, lautet das mehrfach wiederholte Credo des Briefes. ${ }^{58}$

Indem Ambrosius die Vorgänge in Syrien zum legitimen religiösen Konflikt erklärt, kann er die kaiserliche Anordnung zur Wiedererrichtung der Synagoge als gefährlich für Kaiser und Reich stilisieren. Denn Theodosius ' Edikt mache den Bischof von Kallinikon entweder - wenn er nämlich tue, was der Kaiser verlange - zum Verräter an der Kirche oder - wenn er sich weigere - zum Märtyrer für seinen Glauben. Und auch für das administrative Personal, das mit der Durchführung des Edikts betraut werde, also den Comes orientis und die Soldaten, stelle sich im Prinzip die gleiche Alternative. Mit einem Befehl, der gegen die Maximen der Religion verstoße, riskiere Theodosius also die Aufkündigung des Gehorsams durch wesentliche Funktionsträger des Imperiums. In einem Glaubenskonflikt, so macht der Bischof unumwunden klar, bestehe kein regelhafter Anspruch des Kaisers auf Folgeleistung. ${ }^{59}$

Mit seiner Verschiebung der Parameter kann Ambrosius den Kaiser noch an einem anderen neuralgischen Punkt packen: an seiner eigenen Frömmigkeit. Immer wieder im Brief taucht das Motiv des gottesfürchtigen und zutiefst christlichen Herrschers auf. Daran knüpft sich ebenso oft die teils explizite, teils implizite Erwartung, ein solcher Protagonist des rechten Glaubens könne sich doch nicht - zumindest nicht in vollem Bewußtsein - eines Sakrilegs schuldig machen wie es die Begünstigung der jüdischen Christusfeinde wäre. ${ }^{60}$ Auch bei diesem Aspekt des bischöflichen Motivationsprogramms ist es vom wohlmeinenden Ratschlag bis zur unverhohlenen Drohung nur ein kleiner Schritt: Als Gegenbilder des frommen Herrschers bemüht Ambrosius den Apostatenkaiser Julian und den Usurpator Maximus, der, nachdem er ein energisches Edikt gegen das Anzünden einer Synagoge in Rom gerichtet hatte, im Nu und überall die bittersten Niederlagen erlitten habe. ${ }^{61}$ Indem der Bischof die Siege aufzählt, die Theodosius Gott verdanke, ${ }^{62}$ beschwört er somit gleichzeitig die Gefahr für den Kaiser, die göttliche Gnade durch unfromme Akte zu verlieren. Und beim Thema ,göttliche Gnade' angelangt, kann er auch das transzendente Potential der christlichen Religion in die Waagschale werfen. Denn schließlich, so Ambrosius, stehe in dieser Sache

57 Ambr. epist. 74 (40),8: Ambrosius rechtfertigt sich hier theologisch gewunden, weshalb er bislang die Synagoge von Mailand dennoch nicht angezündet habe.

58 Ambr. epist. 74 (40),2, wo er im Rückgriff auf das Alte Testament (Ezech 3,17-19) die Figur des von Gott eingesetzten Wächters bemüht, der die weltlichen Herrscher von Gottes Gebot in Kenntnis setzt, wenn er im Begriff steht zu sündigen; s.a. epist. 74 (40),3; 74 (40),8; 74 (40),2123; 74 (40),28: secundum homines loquor: caeterum plus hominibus Deus timetur, qui etiam imperatoribus iure praefertur. si amico aliquis, si parenti aut propinquo deferendum existimat, recte ego et deferendum Deo, et eum praeferendum omnibus iudicavi.

59 Ambr. epist. 74 (40),6-9; 74 (40), 18.

60 Ambr. epist. 74 (40),21-22.

61 Ambr. epist. 74 (40), 15; 74 (40),23.

62 Ambr. epist. $74(40), 22$. 
nicht nur das innerweltliche Schicksal des Kaisers auf dem Spiel: „Es ist eine ernste Sache“, schreibt er, „,dein Heil für die Juden zu gefährden.“63

An der Argumentation des Bischofs seinem Herrscher gegenüber ist für unsere Fragestellung wiederum nicht der zweifellos besonders rücksichtslose Ton interessant, sondern die Tatsache, daß es sich in der Sache um topische Muster und gerade nicht um Idiosynkrasien des Ambrosius gehandelt hat. Was er in der Kallinikon-Affäre vorbrachte, spiegelt vielmehr Positionen und Handlungsmaximen, die immer wieder wesentliche Optionen des christlichen Umgangs mit Heterodoxen und dem Phänomen der Gewalt bezeichnen. ${ }^{64}$ Wenn aber diese Axiome Plausibilität beanspruchen konnten, waren die beschriebenen Mechanismen imperialer Gewalthegung in der Tat prinzipiell außer Kraft gesetzt, die Neutralität der Institutionen ebenso wie die Macht des Reiches als Grundlage der Ordnung.

Erklärte man Gottes Gesetze - oder vielmehr die partikulare Auslegung davon - zum Maßstab für das eigene Handeln, wurde eine reichsweit verbindliche und wirksame Objektivierung von Richtig und Falsch unmöglich. Und mit der Kategorie des jenseitigen Heils griff auch der Terror nicht mehr, der das Rückgrat der Machtordnung des Reiches gewesen war und die Untertanen von allzu subjektiven Auslegungen abgehalten hatte. Die Entschlossenheit, notfalls für das ewige Leben sterben zu wollen, überforderte die Leistungsfähigkeit weltlicher Zwinggewalt in ihrem Kern. Aus dieser Perspektive war das sakralisierte Märtyrertum der christlichen Kirche die perfekte Negierung des Machtarguments. ${ }^{65}$ Den strukturellen Plausibilitätsverlust von Macht und Terror kann man in der Tat als Paradigmenwechsel begreifen.

Mindestens ebenso folgenreich für die Gewaltfrage war die neue persona des christlichen Kaisers seit Konstantin. Wenn der Herrscher eine - oder die zentrale - Rolle in der neuen religiösen Massenbewegung spielen wollte, durfte er sich den inhaltlichen Anforderungen des Glaubens nicht vollständig verschließen. ${ }^{66}$ Das galt natürlich auch für Normen und Konfliktkonstellationen, die das Christentum transportierte, und blieb naturgemäß nicht ohne Auswirkungen auf seine Haltung zur Gewalt. Denn sich als frommer Herrscher auf den Standpunkt zu stellen, die Gründe für Gewaltanwendung seien vollständig irrelevant für die Beurteilung des Vorgangs, ging kaum mehr an. Der Kampf für den rechten Glauben, für die Bekehrung Paganer etwa oder gegen den verderblichen Einfluß von Häretikern oder Juden durfte einfach nicht dasselbe sein wie gewöhnliches Brigantentum.

63 Ambr. epist. 74 (40), 25-26: grave est fidem tuam pro Iudaeis periclitari (26; Übers. U. Gotter).

64 Vgl. GADDIS 2005, 21-22; zum Einsatz von Gewalt und ihrer Rechtfertigung s. MCMULLEN 1984,86-101; zum „holy man“ als Anführer gewaltsamer Aktionen GADDIS 2005, 151-207.

65 Dazu BRown 1992, 64-65; DIEFENBACH 2000, 112-126, bes. 118-119; GADDIS 2005, 9; 35-45; zur Sorge staatlicher Institutionen, bei der Wiederherstellung der öffentlichen Ordnung Märtyrer zu schaffen GADDIS 2005, 220.

66 Vgl. DiEFENBACH, 1996; MEIER 2007. 
$\mathrm{Da} ß$ daraus ein ernstes Problem für die Autorität des Kaisers und die Stabilität des Imperiums erwachsen würde, war dem Archegeten der Allianz zwischen Christentum und römischem Staat sicherlich nicht bewußt gewesen. ${ }^{67}$ Als Konstantin der Große in das Christentum investiert hat, meinte er wohl durchaus, mit dem neuen privilegierten Kult ließe sich auch die römische Monarchie wirksam sanieren. ${ }^{68}$ Doch mußten die Herrscher des 4. Jahrhunderts bald erfahren, daß ihre zunehmend unausweichlicher werdende imago als christliche Kaiser sie zu Geiseln des religiösen Arguments machte. Der Fall Theodosius zeigt, daß dies je mehr galt, je orthodoxer und frommer ein Herrscher zu erscheinen versuchte. ${ }^{69}$

\section{Christliches Imperium und religiöser Konflikt}

Die christlichen Kaiser befanden sich in der Tat in einem unauflösbaren Dilemma: Einerseits konnten sie - ebensowenig wie ihre paganen Vorgänger - kein Interesse an dezentraler Gewaltanwendung und lokalen Unruhen haben. $\mathrm{Da}$ auch die Herrscher des 4. und 5. Jahrhunderts n. Chr. in diesen Fragen tatsächlich in traditionellen Bahnen dachten, zeigt eine Vielzahl an gesetzlichen Regelungen und Verwaltungsakten. ${ }^{70}$ Das galt auch und gerade für den Einsatz von Gewalt gegen Kultgruppen und ihre Gebäude, weil damit - zumal im Osten - kollektive Frontstellungen entstanden bzw. zementiert wurden, die wiederum rücksichtslos ausgetragene Konflikte auf lokaler Ebene endemisch machten. Auf der anderen Seite aber gab es im christianisierten Imperium kein wirksames Mittel und plausibles Ideologem mehr, um das Gefahrenpotential dieser Konstellation grundsätzlich in Schach zu halten. Gegen Brandstiftung mit religiösen Dogmen war man nahezu wehrlos. Und mehr noch: Die plausibelsten religiösen Konflikte, Gewaltanwendungen gegen Kultorte und Kultgruppen, wurden zur Speerspitze der Bewegung, die das römische Reich grundlegend und nachhaltig veränderte. ${ }^{71} \mathrm{Im}$ Windschatten der Auseinandersetzungen um die sakrale Topographie konnten religiöse Charismatiker und lokale kirchliche Würdenträger in Positionen einrücken, die ursprünglich auf die imperiale Zentrale bezogen waren. Es läßt sich

$67 \mathrm{Zu}$ den unvorhergesehenen Konflikten, die sich aus der staatlichen Involvierung in die christliche Religionspolitik ergaben vgl. POTTER 2004, 402-435; MITCHELL 2007, 276-295.

68 Es ist hier kaum der Ort, die Motive Konstantins für die Aufwertung des Christentums im Reichsverband und die Frage der persönlichen Glaubensüberzeugungen des Kaisers zu diskutieren (s. dazu mit einem Überblick über die aktuelle Forschung JUST 2003, 134-136). Daß die religionspolitischen Maßnahmen Konstantins neben allem anderen zumindest auch einen funktionalen Aspekt hatten, erscheint mir allerdings evident: s. dazu BLEICKEN 1992, 20-52; BRINGMANN 1995; JUST 2003 bes. 157-158; BERRENS 2004, 242 (aus der Perspektive des Solkultes); DRAKE 2000, 235-272; VAN DAM 2007, 293-309.

69 KOLB 1980, bes. 63-64.

70 S.o. n. 21.

71 S. hierzu HAHN - EMMEL - GOTTER 2008 und die anderen Beiträge im vorliegenden Band. 
zeigen, daß die Bischöfe zwischen dem 4. und dem 6. Jahrhundert Funktionen übernahmen, die früher von städtischen Beamten versehen wurden. ${ }^{72} \mathrm{Im}$ selben Zeitraum verschwinden die Repräsentanten der traditionellen zivischen Selbstverwaltung aus den Annalen, und dies ist wohl nicht nur eine Frage von Quellengattungen. Denn mit den religiösen Umwälzungen der Spätantike schwand die Basis ihrer sozialen und politischen Macht. Durch seine privilegierte Möglichkeit, die Menge über religiöse Inhalte - auch für gewaltsame Auseinandersetzungen zu mobilisieren, wuchs dem Bischof ein Instrument zu, das lokal und regional ohne Konkurrenz war. Besonders in den großen Städten des Ostens war damit Politik gegen den Führer der christlichen Gemeinschaft kaum mehr möglich. ${ }^{73}$ So war es im Grunde nur folgerichtig, daß dem Bischof seit dem 4. Jahrhundert schrittweise auch formale Kompetenzen übertragen wurden, die ihn zum eigentlichen Stadtherren machten. ${ }^{74} \mathrm{Da}$ dies wiederum die Art der Kommunikation zwischen der Zentrale und den lokalen Einheiten beeinflußte, liegt auf der Hand. Aus dieser Perspektive ist die religiöse Gewalt unter christlichen Auspizien der Katalysator des Neuen in den Städten des Imperiums.

Gewaltausübung im römischen Imperium war also durchaus ein struktureller Faktor der neuen Verbindung von Staat und Religion und damit der christlichen Religion in der Spätantike selbst. Mit dieser Feststellung, die sich als im Kern empirisch versteht, ist zwangsläufig die jüngst von Jan Assmann losgetretene Debatte um den Zusammenhang von Monotheismus und Gewalt evoziert. ${ }^{75}$ Kennzeichen der Kontroverse um die ,mosaische Unterscheidung', wie Assmann die religiös fundamentierte kategoriale Differenz zwischen ,Wahr' und ,Falsch' nennt, ${ }^{76}$ ist neben der großen Emotionalität, mit der sie geführt wird, die geringe Spezifität der verwendeten Kategorien: Monotheismus, Polytheismus, Gewalt, Toleranz sind allesamt Begriffe von großer und im einzelnen kaum kontrollierbarer Reichweite. Aus der Perspektive des Historikers ärgerlich ist dabei vor allem die Vermischung von heuristischen Ebenen, die vornehmlich theologische Artikel zu diesem Thema kennzeichnet: So ist die Frage nach den tatsächlichen gewaltsamen Konsequenzen des christlichen religiösen Diskurses natürlich unabhängig von der Frage nach der Notwendigkeit von Gewalt im Lichte der christlichen Glaubenssätze. Insbesondere die Argumentationsfigur, Gewalt sei nichts als

72 Vgl. dazu Brown 2002, 45-73; für Gallien JusSEN 1995, 673-713; zur Angleichung des äußeren Erscheinungsbildes der Bischöfe an die weltlichen Amtsträger s. HAENSCH 2003, 124-136.

73 HAHN 2004, bes. 276-280; ISELE 2008 (zu Alexandria).

74 BROWN 1992, 146-152, BAUMGART 1995; MARKSCHIES 1998, 451-469.

75 ASSMANN 2003 mit Diskussionsbeiträgen von Rolf Rendtorff (193-207), Erich Zenger (209220), Klaus Koch (221-238), Gerhard Kaiser (239-271), Karl-Josef Kuschel (273-286); s.a. Palmer 2007 sowie FÜRST, der das Thema Monotheismus und Gewalt in den letzten Jahren in einer ganzen Reihe von teilweise baugleichen Aufsätzen angegangen ist: 2004; $2006 \mathrm{a} ; 2007$.

76 ASSMANN 2003, bes. 28-37. 
ein „Mißbrauch“ der christlichen Religion, ${ }^{77}$ ein kontingenter Betriebsunfall also, transferiert die Diskussion vom deskriptiven in den normativen Bereich. Denn die Kategorie des „Mißbrauchs“ spiegelt einen emphatischen Begriff von der christlichen Religion, keinen analytischen. Zur Widerlegung der Relation zwischen Christianisierung und Gewaltanwendung taugt sie daher nicht. Nachdem Voltaires ,écrasez l'infâme' zurecht aus der Mode gekommen ist, sollte man sich allerdings vor allzu platten Gleichsetzungen hüten: So war Gewaltanwendung offenbar kein gleichmäßig präsentes Epiphänomen frühchristlicher religiöser Kommunikation. $\mathrm{Ob}$ es in den Kommunen des Reiches zu gewaltsamen Auseinandersetzungen kam oder nicht, hing entscheidend von der lokalen Konstellation ab. ${ }^{78}$ Religiöse Gewalt war e in e Handlungsoption - nicht mehr, aber auch nicht weniger. Und auch wenn sich nicht bestreiten läßt, daß es eine Korrelation zwischen Gewaltanwendung und Christianisierung gibt, läßt sich nicht ohne weiteres argumentieren, daß die neue monotheistische Religion der privilegierte Produzent von Gewalt im Imperium Romanum gewesen wäre. ${ }^{79}$ Denn schließlich war das Reich nicht gerade eine politische Idylle, ein Hort weltweiten friedlichen Miteinanders, sondern eine Ordnung, die nach Innen auf struktureller (sozialer) und angedrohter (politischer) Gewalt basierte und nach Außen seine Stabilität über zahlreiche Kriege und kontinuierlichen militärischen Terror aufrechterhielt.

Will man schiefe Verallgemeinerungen vermeiden, ist daher - eher als der Begriff des Monotheismus $-{ }^{80}$ der Begriff der Gewalt zu präzisieren, der meines Erachtens allzu amorph verwendet wird. Denn was sich mit der Christianisierung des Reiches verbinden läßt, ist wohl nicht so sehr die Zunahme von Gewalt insgesamt - eine übrigens schwer quantifizierbare Größe -, und auch nicht die gewaltsame Erschließung des potentiell religiösen Feldes insgesamt. So gab es wohl nur wenige gewaltsame Bekehrungen, ${ }^{81}$ und die Konstruktion auswärtiger Konflikte als Religionskriege war zumindest nicht sehr plausibel. Charakteristisch war dagegen die Zunahme einer ganz spezifischen Form von Gewalt, nämlich die des inneren Zwistes, der stasis. Gängige Aggregatszustände solcher Konflikte waren einerseits Auseinandersetzungen zwischen Christen und Paganen um heilige Orte, aber auch Kämpfe zwischen unterschiedlichen christlichen Bekenntnisgruppen und sogar - als Epiphänomene von Bischofsdoppelwahlen - innerhalb derselben

77 FÜRST 2004, 529-530; 2006, 338; 2006a, 79; 2007, 254; tendentiell auch Zenger bei ASSMANN 2003, 220. Bezeichnend für FÜRSTs Umgang mit der Debatte sind Verharmlosungen, etwa die Überschrift in 2006, 63: „Christliche Schwierigkeiten mit religiöser Pluralität“; ähnlich 2006, 60: „Aus dem Gesagten ergibt sich, daß bei der Christianisierung der Spätantike die Anwendung von Gewalt zwar nicht selten bezeugt ist, aber keine zentrale Rolle spielte (...).

78 Hierin liegt ein ganz wesentlicher Ertrag der Arbeit von Johannes HAHN (2004, pointiert 292294).

79 Zur urbanen Gewalt in der Spätantike als Breitenphänomen s. etwa GREGORY 1983, 138-147; zum Phänomen des religiösen Konflikts: 147-155.

80 Etwa FÜRST 2005.

81 Vgl. FÜRST 2006, 50-59. 
konfessionellen Gruppe. ${ }^{82}$ Katalysatoren des Gewalteinsatzes im Innern waren in der Regel symbolische Akte wie Angriffe auf Kultstätten, die Verhöhnung sakraler Güter sowie Prozessionen durch die Wohngebiete der jeweiligen heterodoxen Gemeinschaft. $^{83}$

Aus imperialer Perspektive bedeuteten Praktiken dieser Art einen schwer zu überschätzenden Einschnitt. Denn offenbar wurden mit der Christianisierung des Imperiums Konflikte, deren gewaltsame Austragung von der römischen Administration über Jahrhunderte gehegt bzw. unterdrückt worden waren, wieder führbar. Im christlich-religiösen Diskurs fand die Konkurrenz zwischen dem lokalen Führungspersonal erneut stabile und binäre Kristallisationspunkte, um die sich eine gewaltbereite Gefolgschaft organisieren ließ ${ }^{84}$ Zugespitzt könnte man formulieren, daß die östlichen Gemeinschaften unter christlichen Auspizien zu ihrer konfliktuellen Identität vor dem römischen Imperium zurückfanden. Aus diesem Blickwinkel wäre die religiöse Gewalt nicht Katalysator des Neuen, sondern die Restauration älterer kultureller Praktiken im neuen Gewand. Von dieser Zäsur war jedoch nicht nur das Zusammenleben der heterodoxen Gruppen im Reich betroffen, sondern auch das Verhältnis des Kaisers zu seinen Untertanen insgesamt. Weit davon entfernt, das monarchische System automatisch zu stärken, ${ }^{85}$ waren Frömmigkeit und christlicher Glaube ein neues Feld, auf dem sich der Herrscher zu beweisen hatte, auf dem er Akzeptanz gewinnen, aber in mindestens gleichem Maße auch verlieren konnte. Denn was fromm und rechtgläubig war, darüber gab es reichsweit durchaus verschiedene Auffassungen, und es zeigte sich rasch, daß der Kaiser keine probaten Mittel besaß, die eigene dogmatische Position, wenn er denn eine hatte, auf breiter Front durchzusetzen. Das heißt aber konkret, daß dem Herrscher der Gehorsam auch ganz handgreiflich aufgekündigt werden konnte. ${ }^{86}$ In dieser Sache gab es für die Zentrale keinen Königsweg. Am ehesten noch ließ sich die eigene Herrschaft imprägnieren, indem der Kaiser selbst den religiösen Diskurs zu besetzen suchte und eine orthodoxe persona pflegte. ${ }^{87}$ Besonderer Ausweis davon waren die markante Anti-Paganen- und Anti-Häretiker-Gesetzgebung ${ }^{88}$ bereits der Söhne Konstantins, vor allem aber der Kaiser seit Theodosius I. Spätestens an diesem Punkt verwickelte sich der Herrscher indessen notwendigerweise in Inkohärenzen zwischen formaler Ordnungs-

82 Alle diese Varianten treten etwa in der Geschichte Antiocheias im 4. Jahrhundert zutage (s. HAHN 2004, 121-189).

83 S. HAHN 2008; ISELE 2008.

84 BROWN 1998, 643-651 und vor allem LiM 1963, passim, der zeigt, wie intellektuelle theologisch-philosophische Positionen durch ihre Implantierung ins Feld des allgemeinen Glaubens ganz unmittelbar politische Wirkung entfalten und zur Mobilisierung von militanten Anhängern taugen; s.a. GOTTER 2003, 205-211 für ein lokales Biotop.

85 So aber FOWDEN 1993.

86 GADDIS 2005, 151-207.

87 MEIER 2007 (für Theodosius II.) und MEIER 2003 (zu Justinian).

88 S. NOETHLICHS 1986, 1155-1176; 1182-1190. 
politik und materiell-dogmatischer Religionspolitik: Eine glaubwürdige Neutralität ließ sich in der Konsequenz kaum noch behaupten. Die Frage nach der Rechtgläubigkeit war für den Herrscher damit endgültig zum Bezugspunkt seiner Macht und die neue Religion zur Grammatik des soziopolitischen Systems geworden. ${ }^{89}$

Versucht man vor diesem Hintergrund abschließend die Veränderung des Verhältnisses von Religion und Politik in der Spätantike auf den Punkt zu bringen, könnte man auf den ersten Blick meinen, in der Ausweitung des religiösen Feldes das Signum des Zeitalters zu sehen. Doch angesichts der früheren ubiquitären Präsenz ,paganer', polytheistischer Religionsübung erscheint dies kaum haltbar. Denn gerade in den lokalen Kulturen des Reiches ebenso wie auf imperialer Ebene waren religiöse Handlungen im politischen und sozialen Leben der vorchristlichen Zeit gerade omnipräsent. Die Idee eines religionsfreien politischen Raumes stand daher auch in vorchristlicher Zeit nicht zur Verfügung. Wozu man pagane Kulte allerdings nicht nutzen konnte, war die Produktion, Anstachelung und Organisation von inneren Konflikten. Die Logik der paganen Religion war auf die Herstellung von kollektiver Einheit gerichtet, nicht auf Dissoziation. Unter diesen Umständen scheint sich mir das Verhältnis von Politik und Religion in der Spätantike wesentlich dadurch zu verschieben, daß die christliche Religion ein konkurrenzlos effizientes Medium des inneren Konflikts war. Diese konfliktuelle Potenz des Christentums bei gleichzeitiger starker Präsenz religiöser Akte hatte eine enorm expansive Tendenz. Denn es läßt sich zeigen, daß seit dem 4. Jahrhundert sämtliche denkbaren Auseinandersetzungen als Auseinandersetzungen zwischen unterschiedlichen religiösen Positionen und Gruppen verstehbar und inszenierbar waren. Diese Beobachtung hat meines Erachtens wiederum unvermeidliche Konsequenzen für eine praktikable Definition von ,religiösem Kon-

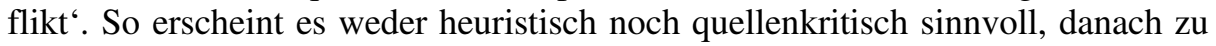
fragen, ob es bei dieser oder jener Auseinandersetzung um eine ,wirklich religiöse' Sache gegangen sei oder ob man die Religion lediglich vorgeschoben habe. Da der Historiker an die individuelle Psyche der spätantiken Akteure nicht mehr herankommt, ist eine essentialistische Unterscheidung zwischen einer genuin religiösen Überzeugung und einer lediglich sekundären religiösen Intention nicht zu treffen und sollte daher - als heuristischer Irrealis - auch nicht das Ziel der Analyse bilden. Verläßlichere Größen sind dagegen kollektive Handlungen und ihre kommunikativen Voraussetzungen. Wenn man akzeptiert, daß sich Gewalt unter christlichen Auspizien - entgegen der imperialen Tradition - religiös legitimieren ließ, sollte man konsequenterweise jede Auseinandersetzung, in der die Parteiungen mit religiösen Argumenten mobilisiert wurden, als religiösen Konflikt oder Religionskrieg ${ }^{90}$ verstehen. Auf dieser Ebene läßt sich Geschehen wohl

89 Vgl. grundsätzlich DIEFENBACH 1996.

90 Es ist im Grunde erstaunlich, daß eine Theorie des religiösen Konflikts, zumindest im Rahmen der empirisch arbeitenden Geschichtswissenschaft, weitgehend unterdeterminiert bleibt - selbst 
noch weitgehend einvernehmlich beschreiben. Jenseits der kommunikativen Oberfläche sozialen Handelns allerdings beginnt rasch die rekonstruktive Willkür.

für Epochen, die traditionell mit den entsprechenden Begrifflichkeiten arbeiten. Dies mag auch damit zusammenhängen, daß in der modernen Konfessionalisierungsforschung der religiöse Konflikt eher als Generator und Subsystem von Staatenbildungsprozessen analysiert wird (s. etwa BURCKHARDT 1997, bes. 548-555). Wie wenig befriedigend der Diskusionsstand ist, zeigen etwa die Versuche Konrad REPGENs, die Natur eines Religionskrieges bzw. religiösen Konflikts in der frühen Neuzeit gleichsam legalistisch über die normativen und öffentlichen Rechtfertigungen des Konfliktbeginns zu definieren (REPGEN 1985; 1987). Dieses Unternehmen bleibt weitgehend im Terminologischen stecken, was REPGEN im übrigen selbst sieht, wenn er den Straßburger Historiker und Juristen des 17. Jahrhunderts, Johann Heinrich Boecler, zustimmend in dem Sinne zitiert, daß sehr zwischen Begründung und Motiv zu unterscheiden sei $(1985,46)$. 


\section{Literaturverzeichnis}

Assmann, J., Die Mosaische Unterscheidung oder der Preis des Monotheismus, München - Wien 2003.

Baltrusch, E., Die Juden und das Römische Reich. Geschichte einer konfliktreichen Beziehung, Darmstadt 2002.

Barrett, J.C., Romanization. A Critical Comment, in: D.J. Mattingly (Hrsg.), Dialogues in Roman Imperialism. Power, Discourse, and Discrepant Experience in the Roman Empire, Portsmouth 1997, 51-64.

Baumgart, S., Die Bischofsherrschaft im Gallien des 5. Jahrhunderts. Eine Untersuchung zu den Gründen und Anfängen weltlicher Herrschaft der Kirche, München 1995.

Bendlin, A., Eine Zusammenkunft um der religio willen ist erlaubt..."? Zu den politischen und rechtlichen Konstruktionen von (religiöser) Vergemeinschaftung in der römischen Kaiserzeit, in: H.G. Kippenberg - G.F. Schuppert (Hrsg.), Die verrechtlichte Religion. Der Öffentlichkeitsstatus von Religionsgemeinschaften, Tübingen 2005, 65107.

Benvicenni, A., Progetti di riforme costituzionali nelle epigrafiche greche dei secoli IV-II a.C., Bologna 2003.

Berrens, St., Sonnenkult und Kaisertum von den Severern bis zu Constantin I. (193-337 n. Chr.), Stuttgart 2004.

Bleicken, J., Constantin der Große und die Christen. Überlegungen zur konstantinischen Wende, München 1992.

Brenk, B., Die Umwandlung der Synagoge von Apamea in eine Kirche. Eine mentalitätsgeschichtliche Studie, in: Tesserae. Festschrift J. Engemann, Münster 1991, 1-25.

Bringmann, K., Hellenistische Reform und Religionsverfolgung in Judäa, Göttingen 1983.

Bringmann, K., Die konstantinische Wende. Zum Verhältnis von politischer und religiöser Motivation, Historische Zeitschrift 260 (1995), 21-47.

Brown, P., Power and Persuasion in Late Antiquity. Towards a Christian Empire, Madison 1992.

Brown, P., Christianization and Religious Conflict, in: A. Cameron - P. Garnsey (Hrsg.), The Cambridge Ancient History XIII: The Late Empire A.D. 337-425, Cambridge 1998, 632-664.

Brown P., Poverty and Leadership in the Later Roman Empire, Hanover 2002.

Burckhardt, J., Die Friedlosigkeit der frühen Neuzeit. Grundlegung einer Theorie der Bellizität Europas, in: Zeitschrift für historische Forschung 24 (1997), 509-574.

Cartledge, P., The Greeks. A Portrait of Self and Others, Oxford 1993.

Dahlheim, W., Geschichte der römischen Kaiserzeit, München ${ }^{3} 2003$.

Deininger, J., Der politische Widerstand gegen Rom in Griechenland 217-86 v. Chr., Berlin - New York 1971.

Diefenbach, St., Frömmigkeit und Kaiserakzeptanz im frühen Byzanz, Saeculum 47 (1996), 35-66.

Diefenbach, St., Jenseits der „Sorge um sich“. Zur Folter von Philosophen und Märtyrern in der römischen Kaiserzeit, in: P. Burschel u.a., Das Quälen des Körpers. Eine historische Anthropologie der Folter, Köln u. a. 2000, 99-131.

Dössel, A., Die Beilegung innerstaatlicher Konflikte in den griechischen Poleis vom 5.-3. Jahrhundert v. Chr., Frankfurt/M. 2003. 
Fowden, G., Bishops and Temples in the Eastern Roman Empire A.D. 320-435, JThS (1978), 53-78.

Fowden, G., Empire to Commonwealth. Consequences of Monotheism in Late Antiquity, Princeton 1993.

Fürst, A., Monotheismus und Gewalt. Fragen an die Frühzeit des Christentums, in: Stimmen der Zeit 222 (2004), 521-531.

Fürst, A., Christentum im Trend. Monotheistische Tendenzen in der späten Antike, in: Zeitschrift für antikes Christentum 9 (2005), 496-523.

Fürst, A., Monotheismus und Monarchie. Zum Zusammenhang von Heil und Herrschaft in der Antike, in: Theologie und Philosophie 81 (2006), 321-338.

Fürst, A., Friedensethik und Gewaltbereitschaft. Zur Ambivalenz des christlichen Monotheismus in seinen Anfängen, in: A. Fürst (Hrsg.), Friede auf Erden? Die Weltreligionen zwischen Gewaltverzicht und Gewaltbereitschaft, Freiburg u.a. 2006a, 45-81. 188-196.

Fürst, A., Wahrer Gott - wahre Gerechtigkeit. Politische Implikationen des Monotheismus in der Spätantike, in: G. Palmer (Hrsg.), Fragen nach dem einen Gott. Die Monotheismus-Debatte im Kontext, Tübingen 2007, 251-282.

Gaddis, M., There is No Crime for Those Who Have Christ. Religious Violence in the Christian Roman Empire, Berkeley u.a. 2005.

Garnsey, P., The Criminal Jurisdiction of Governors, JRS 58 (1968), 51-59.

Gehrke, H.-J., Stasis. Untersuchungen zu den inneren Kriegen in den griechischen Staaten des 5. und 4. Jahrhunderts v. Chr., München 1985.

Gehrke, H.-J., Die Griechen und die Rache. Ein Versuch in historischer Psychologie, Saeculum 38 (1987), 121-149.

Gehrke, H.-J., Geschichte des Hellenismus, München ${ }^{3} 2003$.

Gotter, U., Thekla gegen Apoll. Überlegungen zur Transformation regionaler Sakraltopographie in der Spätantike, Klio 85 (2003), 189-211.

Gotter, U., Fundamental Differences and Cross-Cultural Contact. The Semantics of Power in Greece and Rome, Harvard Studies in Classical Philology 2008, 179-230.

Goodman, M., Opponents of Rome: Jews and Others, in: in: L. Alexander (Hrsg.), Images of Empire, Sheffield 1991, 222-238.

Gregory, T.E., Urban Violence in Late Antiquity, in: R.T. Marchese (Hrsg.), Aspects of Graeco-Roman Urbanism, Oxford 1983, 138-161.

Groß-Albenhausen, K., Imperator Christianissimus. Der christliche Kaiser bei Ambrosius und Johannes Chrysostomus, Frankfurt/M. 1999.

Haensch, R., Römische Amtsinhaber als Vorbilder für die Bischöfe des 4. Jahrhunderts?, in: L. de Blois - P. Erdkamp - O. Hekster - G. de Kleijn - St. Mols (Hrsg.), The Representation and Perception of Roman Imperial Power. Proceedings of the Third Workshop of the International Network Impact of Empire (Roman Empire, c. 200 B.C.-A.D. 476), Amsterdam 2003, 117-136.

Hahn, J., Gewalt und religiöser Konflikt. Studien zu den Auseinandersetzungen zwischen Christen, Heiden und Juden im Osten des Römischen Reiches (von Konstantin bis Theodosius II.), Berlin 2004.

Hahn, J., The Conversion of the Cult Statues: The Destruction of The Serapeion and the Transformation of Alexandria into the 'Christ-Loving' City, in: J. Hahn - St. Emmel - U. Gotter (Hrg.), From Temple to Church. Destruction and Renewal of Local Cultic Topography in Late Antiquity, Leiden 2008, 335-365. 
Hahn, J. - Emmel, St., - Gotter, U. (Hrsg.), From Temple to Church. Destruction and Renewal of Local Cultic Topography in Late Antiquity, Leiden 2008.

Halfmann, H., Die Selbstverwaltung der kaiserzeitlichen Polis in Plutarchs Schrift Praecepta gerendae rei publicae, Chiron 32 (2002), 83-95.

Hansen, M.H., The Athenian Democracy in the Age of Demosthenes. Structures, Principles and Ideology, Oxford 1991.

Heather, P., The Fall of the Roman Empire, London 2005.

Hengel, M., Die Zeloten. Untersuchungen zur jüdischen Freiheitsbewegung in der Zeit von Herodes I. bis 70 n. Chr., Leiden - Köln ${ }^{2} 1976$.

Hengel, M., Jerusalem als jüdische und hellenistische Stadt, in: M. Hengel, Judaica, Hellenistica et Christiana. Kleine Schriften II, Tübingen 1999, 115-156.

Horstkotte, H.-J., Die Strafrechtspflege in den Provinzen der römischen Kaiserzeit zwischen hegemonialer Ordnungsmacht und lokaler Autonomie, in: W. Eck (Hrsg.), Lokale Autonomie und römische Ordnungsmacht in den kaiserzeitlichen Provinzen vom 1. bis 3. Jahrhundert, München 1999, 303-318.

Isele, B., Religiöse Gewalt, heiliger Raum und christliche Topographie in Alexandria und Konstantinopel (4. Jh.), Münster 2008.

Jacques, F. - Scheid, J., Rom und das Reich in der Hohen Kaiserzeit 44 v. Chr. $-260 n$. Chr., Bd. I: Die Struktur des Reiches, Leipzig 1998.

Jördens, A., Das Verhältnis der römischen Amtsträger in Ägypten zu den „Städten“ in der Provinz, in: W. Eck (Hrsg.), Lokale Autonomie und römische Ordnungsmacht in den kaiserzeitlichen Provinzen vom 1. bis 3. Jahrhundert, München 1999, 141-180.

Jussen, B., Über ,Bischofsherrschaften' und die Prozeduren politisch-sozialer Umordnung in Gallien zwischen ,Antike' und ,Mittelalter', in: Historische Zeitschrift 260 (1995), 673-718.

Just, P., Imperator et Episcopus. Zum Verhältnis von Staatsgewalt und christlicher Kirche zwischen dem 1. Konzil von Nicaea (325) und dem 1. Konzil von Konstantinopel (381), Stuttgart 2003.

Juster, J., Les juifs dans l'Empire Romain. Leur condition juridique, économique et sociale, 2 Bde., New York 1914.

Kolb, F., Der Bußakt von Mailand: Zum Verhältnis von Staat und Kirche in der Spätantike, in: Geschichte und Gegenwart. Festschrift K.D. Erdmann, Neumünster 1980, 4174.

Lendon, J.E., Empire of Honour. The Art of Government in the Roman World, Oxford 1997.

Lim, R., Public Disputation, Power, and Social Disorder in Late Antiquity, Berkeley Los Angeles - London 1995.

Linder, A., The Jews in Roman Imperial Legislation, Jerusalem 1987.

Lintott, A., Violence, Civil Strife and the Revolution in the Classical City 750-330 BC, London - Canberra 1982.

Markschies, Chr., Die politische Dimension des Bischofsamtes im vierten Jahrhundert, in: J. Mehlhausen (Hrsg.), Recht-Macht - Gerechtigkeit, Gütersloh 1998, 438-469.

Mason, St., Excursus II: The Deliberative Speech of Agrippa II, in: S. Mason (Hrsg.), Flavius Josephus: Translation and Commentary, vol. 1b: The Judean War, Leiden 2008 (im Druck).

Mason, St., The Greeks and the Distant Past in Josephus' Judean War, in: G. Gardner - K. Osterloh (Hrsg.), Antiquity in Antiquity, Stuttgart 2008a (im Druck). 
Mattingly, D.J., Dialogues of Power and Experience in the Roman Empire, in: D.J. Mattingly (Hrsg.): Dialogues in Roman Imperialism. Power, Discourse, and Discrepant Experience in the Roman Empire, Portsmouth 1997, 7-26.

McLynn, N.B., Ambrose of Milan. Church and Court in a Christian Capital, Berkeley Los Angeles - London 1994.

McMullen, R., Christianizing the Roman Empire (A.D. 100-400), New Haven - London 1984.

Meier, M., Das andere Zeitalter Justinians. Kontingenzerfahrung und Kontingenzbewältigung im 6. Jahrhundert n.Chr., Göttingen 2003.

Meier, M., Die Demut des Kaisers. Aspekte der religiösen Selbstinszenierung bei Theodosius II. (408-450), in: A. Pecar - K. Trampedach (Hrsg.), Die Bibel als politisches Argument. Voraussetzungen und Folgen biblizistischer Herrschaftslegitimierung in der Vormoderne, München 2007, 135-158.

Meyer-Zwiffelhoffer, E., Politikos archein. Zum Regierungsstil der senatorischen Statthalter in den kaiserzeitlichen griechischen Provinzen, Stuttgart 2002.

Mitchell, St., A History of the Later Roman Empire AD 284-641: The Transformation of the Ancient World, Oxford u.a. 2007.

Noethlichs, K.-L., Die gesetzgeberischen Maßnahmen der christlichen Kaiser des vierten Jahrhunderts gegen Häretiker, Heiden und Juden, Köln 1971.

Noethlichs, K.-L., Heidenverfolgung, in: RAC 13 (1986), 1149-1190.

Nörr, D., Imperium und Polis in der hohen Prinzipatszeit, München 1966.

Nörr, D., Zu den Xenokriten (Rekuperatoren) in der römischen Provinzialgerichtsbarkeit, in: W. Eck (Hrsg.), Lokale Autonomie und römische Ordnungsmacht in den kaiserzeitlichen Provinzen vom 1. bis 3. Jahrhundert, München 1999, 257-302.

Palmer, G., Einleitung: Die 'Realität' des Monotheismus' als politisches Problem der Kulturwissenschaften, in: G. Palmer (Hrsg.), Fragen nach dem einen Gott. Die Monotheismus-Debatte im Kontext, Tübingen 2007, 1-22.

Potter, D.S., The Roman Empire at Bay AD 180-395, London - New York 2004.

Rabello, A.M.R., The Legal Condition of the Jews in The Roman Empire, in: ANRW II 13, Berlin 1980, 662-762.

Rajak, T., Friends, Romans, Subjects: Agrippa II's Speech in Josephus's Jewish War, in: L. Alexander (Hrsg.), Images of Empire, Sheffield 1991, 122-134.

Repgen, K., Kriegslegitimation in Alteuropa. Entwurf einer historischen Typologie, in: Historische Zeitschrift 241 (1985), 27-49.

Repgen, K., What is a ,Religious War', in: E.I. Kouri - T. Scott (Hrsg.), Politics and Society in Reformation Europe. Essays for Sir Geoffrey Elton on his Sixty-Fifth Birthday, Basingstoke 1987, 311-328.

Rilinger, R., Humiliores - Honestiores. Zu einer sozialen Dichotomie im Strafrecht der römischen Kaiserzeit, München 1988.

Safrai, S., Das Zeitalter der Mischna und des Talmuds (70-640), in: H.H. Ben Sasson (Hrsg.), Geschichte des jüdischen Volkes. Von den Anfängen bis zur Gegenwart, München ${ }^{3} 1994,377-470$.

Sartre, A. - Sartre, M., Notables en conflit dans le monde Grec sous le Haut-Empire, Cahiers d'histoire 45 (2000), 507-532.

Schaefer, P., Judeophobia. Attitudes Towards the Jews in the Ancient World, Cambridge/Mass. 1997. 
Stahl, M., Imperiale Herrschaft und provinziale Stadt. Strukturprobleme der römischen Reichsorganisation im 1.-3. Jh. der Kaiserzeit, Göttingen 1978.

Stemberger, G., Juden und Christen im Heiligen Land. Palästina unter Konstantin und Theodosius, München 1987.

Swain, S., Hellenism and Empire. Language, Classicism, and Power in the Greek World, AD 50-250, Oxford 1996.

Trampedach, K., Die Tyrannis als Wunsch- und Schreckbild, in: B. Seidensticker - M. Vöhler (Hrsg.), Gewalt und Ästhetik. Zur Gewalt und ihrer Darstellung in der griechischen Klassik, Berlin - New York 2006, 3-27.

Trampedach, K., Die Hasmonäer und das Problem der Theokratie, in: A. Pečar - K. Trampedach (Hrsg.), Die Bibel als politisches Argument. Voraussetzungen und Folgen biblizitischer Herrschaftslegitimierung in der Vormoderne, München 2007, 3765.

Trombley, F.R., Hellenic Religion and Christianization, C.370-529, 2 Bde., Leiden u.a. 1993/1994.

van Dam, R., The Roman Revolution of Constantine, Cambridge 2007.

Mattingly, D.J. (Hrsg.), Dialogues in Roman Imperialism. Power, Discourse, and Discrepant Experience in the Roman Empire, Portsmouth 1997

Wilker, J., Für Rom und Jerusalem. Die herodianische Dynastie im 1. Jahrhundert n. Chr., Frankfurt/M. 2007.

Wolff, H., ,Administrative Einheiten' in den Nordprovinzen und ihre Beziehungen zu römischen Funktionsträgern, in: W. Eck (Hrsg.), Lokale Autonomie und römische Ordnungsmacht in den kaiserzeitlichen Provinzen vom 1. bis 3. Jahrhundert, München 1999, 47-60. 


\title{
Für die Tempel? \\ Die Gewalt gegen heidnische Heiligtümer aus der Sicht städtischer Eliten des spätrömischen Ostens*
}

\author{
HANS-ULRICH WIEMER
}

\section{Einleitung}

„The emperor is what the emperor does“"1 - diese von Fergus Millar geprägte Formel bringt eine Auffassung des römischen Kaisertums zum Ausdruck, die heutzutage weit verbreitet ist. Die Untertanen römischer Kaiser hätten sich indessen mit der Auskunft, der Kaiser sei das, was er tue, wohl kaum zufrieden gegeben. Denn zum einen zählte für die Untertanen eben nicht bloß, was der jeweils regierende Kaiser tatsächlich tat, sondern ebenso, was er ihrer Ansicht nach tun sollte; sie hatten eine konkrete Vorstellung davon, wie ein Kaiser sich zu verhalten habe, und richteten daher an jeden, der diese Rolle übernahm, bestimmte Erwartungen, die aus einem Idealbild des Kaisers resultierten. ${ }^{2}$ Zum anderen aber war es durchaus nicht selbstverständlich, welche Handlungen überhaupt als Handlungen des Kaisers zu gelten hatten. War der Kaiser für alles verantwortlich, was in seinem Namen geschah? War jedem Gehorsam zu leisten, der sich auf den Willen des Kaisers berief?

Für die städtischen Eliten der östlichen Hälfte des römischen Reiches waren diese Fragen alles andere als rein theoretisch. Ihre praktische Relevanz hatte sich sogar noch erhöht, seit Konstantin im Jahre 324 seinen letzten Rivalen Licinius besiegt und damit die Alleinherrschaft über das Imperium Romanum errungen hatte. Denn Konstantin begann sogleich, die noch vor kurzem verfolgten christlichen Gemeinden des Ostens in ostentativer Weise zu fördern. ${ }^{3}$ Die heidnischen Kulte dagegen sahen sich unversehens auf den Status einer nur noch geduldeten Religion reduziert, auch wenn ein bald nach dem Sieg über Licinius erlassenes

\footnotetext{
* Ich danke Malcolm Errington, Johannes Hahn, Oliver Hihn, Josef Rist und Sebastian SchmidtHofner für die kritische Lektüre des Manuskriptes und den Teilnehmern des Münsteraner Kolloquiums für die anregende Diskussion des Vortrages. Alle Zitate ohne Angabe eines Autornamens beziehen sich auf Libanios. Das Manuskript wurde Ende 2004 abgeschlossen.

1 Millar 1992, 6. Zur Kritik an Millars Petition-and-Response-Modell vgl. neben BLEICKEN 1982 nun die Beiträge in WIEMER 2006.

2 Vgl. dazu etwa STRAub 1939; Wickert 1954; KLOFT 1970; WALlaCE-HADRILL 1981.

3 Vgl. dazu etwa BAYNES 1931; KRAFT 1955, 114-132; BARNES 1981, 224ff.
} 
Verbot heidnischer Kulthandlungen wohl rasch wieder außer Kraft gesetzt wurde. ${ }^{4}$ Die Statusverschlechterung war dennoch gravierend: Konstantin ließ altehrwürdige Heiligtümer der griechischen Welt ausplündern, um seine Neugründung Konstantinopel mit Kunstwerken zu schmücken; ${ }^{5}$ einzelne Heiligtümer wurden mit Billigung, wenn nicht auf Befehl des Kaisers sogar geschlossen und zumindest teilweise zerstört. ${ }^{6}$ Unter Constantius II. wurde es nicht besser: Heiligtümer wurden konfisziert, zerstört oder zweckentfremdet, heidnische Kulthandlungen kriminalisiert. $^{7}$

Die städtischen Eliten mußten sich in dieser Situation neu orientieren. Zwar war das Christentum in vielen Städten der östlichen Reichshälfte eine seit langem vertraute Erscheinung. Aber die städtischen Eliten waren den heidnischen Kulten doch noch immer in vielfältiger Weise aufs engste verbunden. Städtische Heiligtümer und Feste bildeten einen Fokus kollektiver Identität, städtische Priestertümer ein Mittel, um sich im innerstädtischen Wettbewerb um Prestige auszuzeichnen. Der Kaiserkult knüpfte ein symbolisches Band zwischen den Städten und dem Herrscher des Weltreiches. ${ }^{8}$ Unter Konstantin und seinen Nachfolgern wurde dies alles problematisch. Wer sich dem neuen Trend verschloß, riskierte damit Nachteile im Wettbewerb um die kaiserliche Gunst. Nicht wenige waren und blieben noch lange entschlossen, diesen Preis zu zahlen. Wenn sie Macht und Prestige behalten wollten, mußten sie versuchen, das Kaisertum der religiösen Aspekte zu entkleiden, die ihm seit seiner Entstehung anhafteten; für sie mußte der Kaiser über den Religionen stehen.

Man konnte aber natürlich auch mit der Zeit gehen. Wer auf ein für Christen anstößiges Engagement zugunsten heidnischer Kulte verzichtete, verbesserte seine Chancen, in den Genuß kaiserlicher Gunst zu kommen, erheblich. ${ }^{9}$ Diese Aussicht war nicht zuletzt deswegen so verlockend, weil sich unter Konstantin und Constantius ungeahnte Möglichkeiten sozialen Aufstiegs im Dienst des Kaisers eröffneten; ich erinnere lediglich an die Vermehrung der Statthalterposten und die Gründung Konstantinopels. Einige der Tempel, die unter Constantius konfisziert wurden, landeten nachweislich im Besitz von Leuten, die den städti-

4 So inter alios ERRINGTON 1988; WIEMER 1994, 520-523 sowie jetzt M. Wallraff, Die antipaganen Maßnahmen Konstantins in der Darstellung Eusebs, in diesem Band. Anders z. B. BARNES 1984; BRADBURY 1994.

5 Vgl. dazu Dagron 1984, 36-37; 373-376 mit den Belegen. Prägnant Hieron. chron. ad a. 330: Constantinopolis dedicatur paene omnium urbium nuditate.

6 Eus. l.C. 8; v.C. 3,55 (Aphaka); 3,56 (Asklepios in Aigeai; vgl. dazu jedoch WiEMER 1994, 522-523 Anm. 58); 3,58 (Aphrodite in Heliopolis-Baalbek).

7 Die erhaltenen Gesetze (Cod. Theod. 16,10,4-6) stammen zwar alle aus der Zeit nach dem Sieg über Magnentius und beziehen sich sämtlich auf den westlichen Reichsteil, doch zeigen die literarischen Quellen, vor allem Libanios und Julian, daß heidnische Kulthandlungen unter Constantius auch im Osten kriminalisiert wurden. Vgl. dazu vor allem LEPPIN 1999.

8 Dazu grundlegend PRICE 1984.

9 Vgl. dazu HAEHLING 1978 mit den kritischen Bemerkungen von BARNES 1995. 
schen Eliten entstammten. ${ }^{10}$ Andererseits war ein aggressives Vorgehen gegen heidnische Kulte, Feste und vor allem Heiligtümer schwerlich im Sinne der städtischen Eliten. In ihrer übergroßen Mehrheit waren die städtischen Repräsentanten von Bildung und Besitz keineswegs bereit, altbewährte soziale Netzwerke wegen religiöser Differenzen aufs Spiel zu setzen; das Miteinander von Heiden und Christen innerhalb der Elite einer Stadt war im römischen Osten während des 4. Jahrhunderts die Regel und blieb es vielerorts noch lange darüber hinaus.

Eine komplizierte Situation also. Die heidnische Restauration Julians schuf zusätzliche Verunsicherung. In nicht wenigen Städten der östlichen Reichshälfte wurden Tempel neu eröffnet und Kulte wiederbelebt. ${ }^{11}$ Diejenigen, die im Ruf standen, Gewalttaten gegen Heiligtümer verübt oder angestiftet zu haben, mußten um ihren Besitz, ja teilweise sogar um ihr Leben fürchten. ${ }^{12}$ Nach dem Tode Julians sanken die heidnischen Kulte auf den Status einer nur noch geduldeten Religion zurück. Die von Julian restituierten Tempelgüter wurden erneut konfisziert; ${ }^{13}$ bald folgte ein Verbot derjenigen Kulthandlung, die der griechischen Religion seit ihren Ursprüngen ein unverwechselbares Gepräge gegeben hatte: des Tieropfers. ${ }^{14}$ Als im Jahre 379 der Spanier Theodosius den Thron des Ostreiches bestieg, ${ }^{15}$ schien sich die Politik gegenüber den Heiden zunächst nicht wesentlich zu ändern. Zwar hatte man es mit einem frommen Christen zu tun, an dessen persönlichem Bekenntnis es spätestens seit seiner Taufe im Jahre 380 nichts zu rütteln und zu deuteln gab. Doch Theodosius schien die Trennung zwischen seinem persönlichem Bekenntnis und seiner Rolle als Kaiser zunächst durchaus zu respektieren und setzte die integrative Heidenpolitik seines Vorgängers Valens bis weit in die 380er Jahre hinein fort. ${ }^{16}$

Dennoch wurde die Situation des Heidentums unter Theodosius in manchen Teilen der östlichen Reichshälfte zunehmend prekär. Denn seit Julian hatten Bestrebungen, die heidnischen Kulte wenn nötig mit Gewalt zu unterbinden, erheblich an sozialem Boden gewonnen. Militante Gegner des Heidentums erfreuten

10 Vgl. dazu WIEMER 1995b, 65-66; HAHN 2004, 131-132.

11 Vgl. dazu ARCE 1975.

12 Gewalttätige Ausschreitungen gegen Christen blieben allerdings die Ausnahme, und von einer staatlichen Christenverfolgung kann keine Rede sein; so mit Recht jetzt wieder HAHN 2004, 173-177. Indessen bleibt festzuhalten, daß Julian geneigt war, über Gewalttaten gegen Christen hinwegzusehen: WIEMER 1995b, 67 mit Anm. 286.

13 Von dieser Maßnahme zeugen die an Valentinians praefectus praetorio Claudius Mamertinus und den comes sacrarum largitionum Caesarius gerichteten Konstitutionen Cod. Theod. 5,13,3 und 10,1,8. Zur Religionspolitik des Valens vgl. jetzt LENSKI 2002, $211 \mathrm{ff.}$

14 Or. 30,7; vgl. 8; 15; 16; 18. Dieses Verbot ist nur bei Libanios ausdrücklich bezeugt; im ,Codex Theodosianus' hat sich lediglich ein Verbot nächtlicher Opfer erhalten, das an den Prätorianerpräfekten Saturninius Secundus Salutius adressiert ist und wohl der Prävention gegen divinatorische Praktiken diente: Cod. Theod. 9,16,7. Nach Zos. 4,3,2-3 setzte Valentinian I. dieses Verbot auf Bitten des proconsul Achaiae Vettius Agorius Praetextatus für dessen Provinz außer Kraft.

$15 \mathrm{Zu}$ den ersten Regierungsjahren Theodosius' I. vgl. jetzt ERRINGTON 1996; ERRINGTON 1997b; LEPPIN 2003, 35-86.

16 Zur Religionspolitik vgl. jetzt LIZZI 1996; ERRINGTON 1997b; LEPPIN 2003, 67ff., 153ff. 
sich hohen Ansehens in allen Schichten der Bevölkerung, einschließlich von Teilen der städtischen Eliten; wenn sie selbst auch teilweise den Unterschichten entstammten, so hatten sie in den Bischöfen doch einflußreiche Fürsprecher, die inzwischen bereits zum Establishment gehörten. ${ }^{17}$ Je lauter der Wunsch, der Kaiser möge dem widerlichen Kult der Götzen endlich ein Ende machen, geäußert wurde, desto schwieriger wurde es für Theodosius, heidnische Interessen in seiner Politik zu berücksichtigen.

\section{Die Rede „Für die Tempel“}

Wer sich für die Frage interessiert, wie die städtischen Eliten des spätrömischen Ostens diese konfliktträchtigen Entwicklungen bewältigten, kann an Libanios und seiner Rede „Für die Tempel“ nicht vorbeigehen. ${ }^{18}$ Denn in keinem anderen zeitgenössischen Text wird die Gewalt gegen heidnische Heiligtümer einer so eingehenden und so eindringlichen Kritik unterworfen, und das von einem Autor, der dem Ratsherrenstand Antiocheias entstammte und ihm zeitlebens eng verbunden blieb. ${ }^{19}$ Indessen macht es der Text dem modernen Historiker keineswegs leicht. Wer den Wert einer Quelle nach der Menge konkreter Details und dem Grad terminologischer Präzision bemißt, wird herbe enttäuscht. Libanios liebte vage Formulierungen und ausgetretene Gemeinplätze; er mied Eigennamen und termini technici. Doch damit nicht genug: Der Text ist nicht den Prinzipien der antiken Historiographie verpflichtet, sondern denen der Rhetorik. Das aber bedeutet: Eine ausgewogene Darstellung des Sachverhaltes wird in der Rede „Für die Tempel“ gar nicht erst versucht, vielmehr steht bereits die Selektion und Präsentation dessen, was wir Historiker gerne Fakten nennen, im Dienste einer konkreten Wirkungsabsicht, einer rhetorischen Strategie. ${ }^{20}$

Eine der Eigenart der Quelle angemessene Interpretation setzt daher voraus, daß man sich zunächst über intendierte Adressaten, psychagogische Intention und

17 Zur Rolle der Bischöfe im 4. Jahrhundert vgl. neben BROWN 1992 jetzt auch HAHN 2004, 276280.

18 Die erste Ausgabe der Rede besorgte 1634 Jacques Godefroy, der gelehrte Herausgeber des ,Codex Theodosianus'; wiederabgedruckt in GODEFROY 1733, 458-498; vgl. auch die Edition von REISKE 1793, III, 144-204. Die heute maßgebliche Ausgabe wird FÖRSTER 1906, 80-118 verdankt. In jüngerer Zeit hat NORMAN 1977, 91-151 Försters Text mit einer englischen Übersetzung und erklärenden Anmerkungen herausgegeben. Bereits in den dreißiger Jahren des 20. Jahrhunderts hatte LOY 1933 eine französische Übersetzung mit Kommentar veröffentlicht. Eine italienische Übersetzung mit erklärenden Anmerkungen bietet ROMANO 1982. Eine Übersetzung ins Deutsche durch Hans-Günther Nesselrath wird in der Reihe SAPERE erscheinen.

19 Vgl. dazu noch immer PetiT 1955, 17-21; LieBeSChUeTZ 1972, 1-39 mit der Modifikation durch WIEMER 1995a.

20 Dazu grundsätzlich WIEMER 1995b, 367-376. 
argumentative Struktur des Textes Klarheit verschafft. ${ }^{21}$ Auszugehen ist von der Tatsache, daß die Rede „Für die Tempel“ der literarischen Gattung der Beratungsrede, dem genos symbouleutikon, zugehört, die Elemente der Lobrede auf einen Kaiser, des basilikos logos, in sich aufgenommen hat: ${ }^{22}$ der Text gibt sich als eine im Konsistorium gehaltene Ansprache des Sophisten an Kaiser Theodosius. Es steht außer Zweifel, daß es sich hierbei um eine literarische Fiktion handelt; Libanios ist Theodosius niemals begegnet.

Weniger klar und umstritten ist dagegen, wann der Text entstanden ist, wie er verbreitet wurde und ob diese Verbreitung gleich nach der Entstehung begann. ${ }^{23}$ Mit einer Reihe anderer Forscher bin ich der Meinung, daß Libanios die Rede „Für die Tempel“" schrieb, während Maternus Cynegius die Prätorianerpräfektur des Orients innehatte, also vor dem Tod des Präfekten im Jahre 388. Im Gegensatz zu anderen halte ich es jedoch für unvorstellbar, daß der Sophist es gewagt haben würde, die wütenden Angriffe auf Cynegius in irgendeiner Weise zu veröffentlichen, solange dieser in Amt und Würden stand. Mein Lösungsvorschlag lautet, daß Libanios die Rede „Für die Tempel“ zwar in der Absicht schrieb, der Gewalt gegen die heidnischen Heiligtümer entgegenzuwirken, sie aber zunächst unter Verschluß hielt, solange die Umstände ihrer Aufnahme in Konstantinopel denkbar ungünstig waren. Als jedoch der Heide Tatianus 388 die Nachfolge des Cynegius übernahm, schienen für Libanios wieder bessere Zeiten anzubrechen; der Amtsantritt des heidnischen Präfekten dürfte den Sophisten bewogen haben, das Plädoyer „Für die Tempel“ ihm und anderen Gesinnungsfreunden in Konstantinopel zugänglich zu machen. Falls diese Hypothese das Richtige trifft - und sie paßt durchaus zu dem, was wir über die Verbreitung von Libanios' Reden wissen - dann haben wir es bei der Rede „Für die Tempel“ mit einem Lesetext zu tun, der Personen, die bei Theodosius über Einfluß verfügten, mit Argumenten versorgen sollte, die gegen die Zerstörung von Heiligtümern vorgebracht werden konnten.

Tatsächlich zeigt eine Analyse der Rede, daß Libanios sich keineswegs an Adressaten wendet, die von der Überlegenheit des alten Götterkults ebenso überzeugt sind wie er selbst. Vielmehr setzt er ausdrücklich voraus, daß der Kaiser nun einmal Christ ist und sich mit ebensolchen umgibt; aus diesem Grund ist Libanios' Argumentation durchgängig so angelegt, daß sie auch auf Christen zu wirken vermag. Gewiß macht Libanios aus seiner abgrundtiefen Verachtung für die christlichen Mönche, die er als zügellose und ungebildete Menschen niedriger

21 Damit ist zugleich gesagt, weshalb ich die Vorbehalte teile, die FOWDEN 1995 gegenüber einer Quellenlektüre äußert, wie sie etwa bei TROMBLEY 1994, II, 134-142 geübt wird. Methodisch nicht hinreichend reflektiert und daher unergiebig ist auch die Konfrontation ausgewählter Passagen aus der Rede „Für die Tempel“ mit Religionsgesetzen bei WYTZES 1978, zumal dort die Adressaten der einschlägigen Konstitutionen kaum berücksichtigt werden.

22 Zur Annäherung zwischen den beiden Genera in der Spätantike am Beispiel der Rede „Für Aristophanes“ (or. 14) vgl. WIEMER 1995b, 125-127.

23 Vgl. dazu WIEMER 1995a, 123-129. 
Herkunft verspottet, keinen Hehl; er verzichtet jedoch auf Polemik gegen christliche Glaubensinhalte und weist an einer Stelle (§ 29) sogar ausdrücklich darauf hin, daß erzwungene Konversionen im Widerspruch zur Bibel stünden. Libanios' Plädoyer „Für die Tempel“ appelliert an Überzeugungen, die Christen und Heiden gemeinsam waren, vor allem an den Respekt vor dem Gesetz und vor dem Kaiser.

Nun zu Aufbau und Gedankengang der Rede: Nach dem Proömium (§ 1-3) skizziert Libanios zunächst, wie es zur Entstehung von Heiligtümern gekommen sei (§ 4-5): Schon die ersten Menschen hätten den Göttern Heiligtümer und Altäre errichtet; seit es Städte gebe, hätten die Tempel sich über das gesamte Reich ausgebreitet. Er wendet sich dann der Religionspolitik der römischen Kaiser seit Konstantin zu; ich werde auf diesen Abschnitt (§ 6-7) später ausführlicher eingehen. An dieser Stelle genüge die Feststellung, daß Libanios zeigen will, daß Theodosius an der durch Valentinian und Valens geschaffenen Rechtslage nichts geändert habe: Nach wie vor seien zwar Tieropfer verboten, Weihrauchopfer jedoch erlaubt (§ 7-8). Damit ist er bei seinem eigentlichen Thema angekommen, der Gewalt gegen Tempel. Da nämlich Theodosius das Betreten von Tempeln ebensowenig verboten habe wie das Darbringen von Rauchopfern, so folgert Libanios, seien Angriffe auf heidnische Heiligtümer als Rechtsbruch, als Verbrechen, aufzufassen.

Diese Anklage richtet sich zunächst ausschließlich gegen Mönche, die Tempel, Götterbilder und Altäre zerstörten, und zwar weniger in den Städten als vor allem auf dem Lande. Libanios betont, daß diese Gewalttaten nicht bloß den direkt Betroffenen, sondern auch dem Kaiser selbst schadeten. Denn die Zerstörung von Heiligtümern entmutige die Bauern und verringere dadurch das Steueraufkommen (§ 10-11). Zudem raubten die Mönche den Bauern sogar Saatgut und Vieh, ja zum Teil sogar ihr Land, indem sie es fälschlich als heiliges Land deklarierten. Der Bischof Antiocheias aber decke diese Raubzüge, indem er die Täter lobe und die Opfer verhöhne. Aus dieser Darstellung der Sachlage ergibt sich der Appell an den Kaiser, für Recht und Ordnung zu sorgen, was ja das Ziel all seiner Bestrebungen sei. Theodosius dürfe nicht zulassen, daß ein Teil seiner Untertanen einen anderen daran hindere, am ,allgemeinen Glück“ (koina agatha) teilzuhaben, wie Libanios unter Rückgriff auf eine gängige Formel der kaiserlichen Propaganda ${ }^{24}$ formuliert ( $\left.\$ 14\right)$.

Im argumentierenden Teil der Rede setzt Libanios sich mit antizipierten Einwänden auseinander. Die Behauptung, daß die Zerstörung von Heiligtümern als Strafe für Verstöße gegen das Verbot von Tieropfern gerechtfertigt sei (§ 15), ist nach Libanios unbewiesen und unbeweisbar. Gewiß schlachteten die Bauern bei bestimmten Festen Tiere, um sie anschließend zu verspeisen. Aber zwischen dem Schlachten von Tieren und einem Tieropfer sei sorgsam zu unterscheiden, zumal es ja nicht verboten sei, beim Festschmaus die Götter anzurufen und Räucherwerk

24 Wie geläufig der Slogan salus rei publicae auch unter den Nachfolgern Julians war, lehrt ein Blick in den Index V (,Legends“, 317-323, hier: 321) des neunten, von J. W. E. Pearce herausgegebenen Bandes der „Roman Imperial Coinage“ (London 1951). 
zu verbrennen (§ 16-19). Auf den Einwand, die Zerstörung von Heiligtümern sei deswegen gerechtfertigt, weil sie Heiden dazu veranlasse, sich dem Christentum zuzuwenden, entgegnet der Sophist, daß die Opfer der Gewalt an ihrem Glauben nur um so hartnäckiger festhielten. Niemand wisse besser als Theodosius selbst, daß die Zerstörung von Gebäuden keinen Gesinnungswandel bewirken könne; denn sonst hätte der Kaiser die Heiligtümer schon längst zerstören lassen (§ 27). Allenfalls würden Konversionen vorgetäuscht ( $\S 28)$, da sich ein Gesinnungswandel nun einmal nicht erzwingen lasse. Im übrigen verstoße Gewaltanwendung auch gegen Gebote des christlichen Glaubens, den man doch auszubreiten beanspruche $(\S 29) .{ }^{25}$

Der dritte Einwand, den Libanios einer ausführlichen Widerlegung würdigt, lautet, daß die Zerstörung der Heiligtümer der Erde und den Menschen Nutzen bringe und dadurch gerechtfertigt sei (§ 30). Libanios rekurriert zunächst auf die Geschichte - den Aufstieg Roms zur Weltherrschaft, die beiden Trojanischen Kriege und die Perserkriege -, um zu demonstrieren, daß es den Menschen in der Vergangenheit stets genützt habe, die Götter zu verehren (§ 31-32). Der Sophist verweist sodann auf zwei Ausnahmen vom allgemeinen Verbot des Tieropfers, die seiner Ansicht nach belegen, daß der Kaiser den Nutzen des Götterkultes wider Willen selbst anerkennen muß, das Beispiel Roms und das Beispiel Alexandreias. Denn wie Theodosius (§ 33-34) den Götterkult in Rom deshalb nicht antaste, weil er fürchte, die Götter würden dem Reich dann ihre Huld entziehen, so unterbinde er den Kult des Nils deshalb nicht, weil er wisse, daß von seinem ordnungsgemäßen Vollzug die Nilschwelle und damit der landwirtschaftliche Ertrag Ägyptens abhänge (§ 35-36).

Libanios geht schließlich auch der heiklen Frage nicht aus dem Wege, ob es den Kaisern seit Konstantin genutzt oder geschadet habe, wenn sie Maßnahmen für oder gegen den Götterkult ergriffen. Konstantin selbst, so behauptet Libanios, habe schwer dafür gebüßt, daß er die Tempelvermögen konfiszierte, und zwar teils noch zu Lebzeiten, teils erst nach seinem Tode (§ 37). Der Sophist spielt hier vermutlich auf die sogenannte Familientragödie des Jahres 326, die Hinrichtung der Ehefrau Fausta und des Thronfolgers Crispus an ${ }^{26}$ zum anderen auf den gewaltsamen Tod seiner Söhne und Nachfolger Constantius II. und Constans. Das leuchtende Gegenbild ist für Libanios natürlich Julian; ${ }^{27}$ er habe die Perser an den Rand des Untergangs gebracht und dadurch immerwährenden Ruhm erworben ( $\S$ 40-41). An keiner anderen Stelle der Rede mutet Libanios christlichen Ad-

25 Das Problem von Scheinkonversionen unter staatlichem Druck beschäftigte (in der Auseinandersetzung mit den Donatisten) auch Augustinus; vgl. dazu BROWN 1964; auch in: BROWN 1977, 260-278.

26 Die Verwandtenmorde Konstantins erwähnt auch Jo. Chrys. hom. 15,5 in Phil. $(P G$ 62, 295) als eine allgemein bekannte Angelegenheit.

27 Vgl. dazu PETIT 1978, wo jedoch zwischen zeitgenössischen und postumen Äußerungen nicht hinreichend unterschieden wird. Dieses Defizit versucht WIEMER 1995b passim auszugleichen. Vgl. auch MALOSSE 1995. 
ressaten so viel zu wie hier. Er gibt den Perserkrieg Julians, der in einem Desaster geendet hatte, wider besseres Wissen als einen unaufhaltsamen Siegeszug aus, der nur durch einen heimtückischen Anschlag auf den Kaiser habe gestoppt werden können. Immerhin hütet er sich, den Verdacht, daß Julian einem christlichen Komplott zum Opfer gefallen sei, offen auszusprechen.

Auf die mit heidnischer Ideologie befrachtete Apologie Julians folgt ziemlich unvermittelt ein pragmatischer Vorschlag. Nach dem Ausflug auf das kontroverse Feld der Geschichtstheologie versucht Libanios wieder gemeinsamen Boden für Christen und Heiden zu erreichen, indem er die Tempel als Gebäude von hohem ästhetischem Wert verteidigt (§ 42-43). Wer die Städte „bewahren“ wolle - und dies war das erklärte Ziel aller spätrömischen Kaiser, einschließlich des Theodo$\operatorname{sius}^{28}-$, der müsse auch die Tempel bewahren, die doch der größte Schmuck der Städte seien - nach den Kaiserpalästen natürlich, wie der Sophist verschmitzt hinzufügt. Bevor der Kaiser also zulasse, daß die Tempel, die ihm doch ohnehin bereits gehörten, zerstört würden, solle er sie lieber einer anderen Verwendung zuführen, zum Beispiel als Speicher für Steuergetreide.

Der argumentierende Teil der Rede „Für die Tempel“ endet nun freilich nicht mit diesem pragmatischen Vorschlag, sondern mit einer wütenden Attacke auf den christlichen Prätorianerpräfekten Maternus Cynegius, ${ }^{29}$ die von einem konkreten Vorfall, der Zerstörung eines großen Heiligtums an der Reichsgrenze, ihren Ausgang nimmt. Dieses Heiligtum lag, wie im Anhang begründet wird, in Edessa, der Hauptstadt der Provinz Osrhoene (§ 43-44). Libanios' Argumentation zielt an dieser Stelle darauf, dem Präfekten, dessen Namen er taktvoll verschweigt, die alleinige Verantwortung für diese Tat anzulasten. Diese rhetorische Strategie mußte schon deswegen naheliegen, weil Theodosius noch 382 die Schließung eines großen Heiligtums in der Provinz Osrhoene, die unter Berufung auf ein kaiserliches Gesetz erfolgt war, wieder rückgängig gemacht hatte, allerdings unter der Auflage, daß dort keine „verbotenen Opfer“ dargebracht würden. ${ }^{30}$ Als es wenige Jahre später in derselben Provinz zu der von Libanios beklagten Tempelzerstörung kam, hofften die Heiden indessen vergebens auf den Kaiser. Libanios selbst muß implizit einräumen, daß Cynegius keineswegs ohne

28 Eine eingehende Untersuchung der programmatischen Ziele kaiserlichen Handelns gegenüber den Städten und Stadträten ist ein Desiderat der Forschung. Libanios setzt an vielen Stellen ohne weiteres voraus, daß ,gute' Kaiser für Städte und Stadträte Sorge tragen, am nachdrücklichsten im „Epitaphios“ auf Julian (or. 18, bes. 146-150) und in der an Theodosius adressierten Rede „Für die Stadträte“ (or. 49).

29 Zur Person vgl. PLRE I, 235-236 Nr. 3. Daß Cynegius auf einer Reise durch den Osten, die ihn bis nach Ägypten führte, Heiligtümer zerstörte, bezeugen Zos. 4,37,3; Consul. Constant. ad a. 388.

30 Cod. Theod. 16,10,8 (382, Palladio duci Osdroenae): Aedem ... iugiter patere publici consilii auctoritate decernimus neque huic rei obreptivum officere sinimus oraculum. Ut conventu urbis et frequenti coetu videatur, experientia tua omni votorum celebritate servata auctoritate nostri ita patere templum permittat oraculi, ne illic prohibitorum usus sacrificiorum huius occasione aditus permissus esse credatur. 
jede Rückendeckung durch Theodosius gehandelt hatte, wenn er argumentiert, der Präfekt habe Theodosius vorgespiegelt, daß in dem großen Heiligtum Tieropfer dargebracht würden, und dadurch den ansonsten stets zur Milde geneigten Kaiser zornig gemacht ( $\$ 47)$. Zudem erklärt der Sophist, Cynegius und andere lehnten zwar in der Öffentlichkeit jede Verantwortung für ihre Taten ab, indem sie sich als bloße Vollzugsorgane des kaiserlichen Willens ausgäben; wenn sie den Kaiser jedoch unter vier Augen sprächen, rühmten sie sich, ihm und seinem Haus durch die Zerstörung von Tempeln eine Wohltat zu erweisen (\$ 49).

In der Peroratio appelliert Libanios noch einmal an den Kaiser als Quelle und Hüter der Rechtsordnung ( $\$ 52-55$ ). Er betont ausdrücklich, daß es dem Kaiser völlig freistehe, den Götterkult in allen öffentlichen Formen zu verbieten und allen Untertanen den Vollzug des christlichen Kultes zu befehlen und zwar unter Androhung der Todesstrafe (§ 52). Wenn Theodosius dies nicht tue, so behauptet Libanios, dann deswegen, weil er seine Religion zwar für besser halte als diejenige der Heiden, aber das Heidentum eben nicht für ein Sakrileg, das als solches zu bestrafen wäre. Vielmehr habe der Kaiser Heiden sogar durch die Verleihung von Ämtern geehrt und sie zu seinen Tischgenossen gemacht (§ 53). Die Rede endet mit dem Aufruf, der Kaiser solle den Zustand der Rechtsunsicherheit endlich beenden: Entweder solle er die Zerstörung von Tempeln ausdrücklich anordnen; dann werde man dies zähneknirschend hinnehmen. Wenn aber weiterhin Tempel ohne kaiserliche Erlaubnis zerstört würden, dann würden sich die „Eigentümer der Äcker" selbst zu helfen wissen (§ 55).

\section{Die Gewalt gegen heidnische Heiligtümer aus der Sicht des Libanios}

Nachdem wir uns über die rhetorische Strategie des Libanios Klarheit verschafft haben, können wir uns an die historische Auswertung des Textes wagen. Dabei soll hier die Frage im Vordergrund stehen, wie Libanios die Gewalt gegen die heidnischen Heiligtümer konzeptualisiert. Ich möchte dieser Frage unter vier Gesichtspunkten nachgehen: 1) Wo und wie wird Gewalt gegen heidnische Heiligtümer ausgeübt?, 2) Von wem geht diese Gewalt aus?, 3) Welche Rolle spielt der Kaiser? und 4) Wer widersetzt sich der Gewalt?

Zunächst also die Frage nach der Lokalisierung und den Modalitäten der Gewaltausübung. Libanios stellt ausdrücklich und wiederholt fest, daß vor allem, wenngleich nicht ausschließlich, ländliche Heiligtümer zerstört würden. $\operatorname{Im~} \mathrm{Zu}$ sammenhang mit der Zerstörung von Heiligtümern kommt es seiner Darstellung zufolge aber auch zu Übergriffen auf bäuerlichen Besitz: Vorräte und Vieh werden geraubt, Bauern mit der Begründung enteignet, sie bebauten heiliges Land. Libanios gibt den geschilderten Sachverhalt als allgemein bekannt und unbestritten aus; strittig sei lediglich seine Bewertung. Allerdings fällt auf, daß die einzigen konkreten Beispiele, die er anführt, nicht aus dem ländlichen, sondern aus 
dem städtischen Bereich stammen: neben der Zerstörung des großen Heiligtums an der Reichsgrenze diejenige einer Asklepios-Statue in Beroia (\$22-23). Es griffe wohl zu kurz, wollte man diesen Befund damit erklären, daß die städtischen Heiligtümer eben einfach prominenter waren als die ländlichen, auch wenn dies natürlich zutrifft. Vielmehr kommt in der Anonymität, in der die ländlichen Heiligtümer bei Libanios erscheinen, die Fremdheit zum Ausdruck, die der Professor der griechischen Rhetorik bei aller heidnischen Solidarität gegenüber den im semitischen Bereich verwurzelten, ländlichen Kulten Nordsyriens empfand: Wenn Libanios von Zeus sprach, so dachte er an den Olympier, wie Homer ihn schildert, nicht an einen als Altar vorgestellten Zeus Madbachos, wie er auf der Koryphe genannten Anhöhe bei dem Dorf Telanissos (Djebel Sheikh Berekat) verehrt wurde. ${ }^{31}$ Erst recht waren ihm all die in Nordsyrien verehrten Gottheiten fremd, die dem Pantheon des klassischen Griechenland nicht einmal dem Namen nach angeglichen worden waren. ${ }^{32}$

Stellt man die Frage, von wem die Gewalt gegen heidnische Heiligtümer Libanios zufolge ausgeht, so tritt eine merkwürdige Inkohärenz zu Tage. In der Narratio der Rede „Für die Tempel“ zetteln die Mönche die Zerstörung von Tempeln nicht bloß an, sondern führen sie auch selbst aus, wobei die Beteiligung staatlicher Instanzen mit keinem Wort erwähnt wird. Im Schlußteil der Argumentatio wird die Gewalt dagegen von staatlichen Instanzen ausgeübt; Libanios erwähnt die Beteiligung von „Statthaltern“ (archontes), „Generälen“ (strategoi) und „Soldaten“ (stratiotai) und schreibt die politische Verantwortung dem Inhaber der orientalischen Präfektur zu. Die Mönche hingegen erscheinen in diesem Teil nur noch als treibende Kraft hinter den Kulissen, insofern Cynegius lediglich tut, was seine Frau will, diese selbst aber wiederum den Mönchen hörig ist. Libanios hat hier offenkundig von der Freiheit des Rhetors Gebrauch gemacht, je nach psychagogischem Bedarf einzelne Aspekte eines Themas zu verabsolutieren. Bedeutsamer scheint mir die Beobachtung, daß Libanios darauf verzichtet hat, den antiochenischen Bischof als treibende Kraft hinter den Gewalttaten der Mönche darzustellen. Bei Libanios trägt er lediglich insofern eine Mitschuld, als er die Aktionen der Mönche gutheißt; er wird aber an keiner Stelle als Initiator der Gewalt dargestellt.

31 IGLS 465-475 mit Trombley 1994, II, 253-255; Trombley 2004, 60-61 (mit Hinweis auf die in IGLS und SEG nicht enthaltene, von JARRY 1967, 163-164 Nr. 41 publizierte griechische Inschrift für den gemeinsam mit Zeus Madbachos verehrten Gott Salmanes). Der in Telanissos Zeus Madbachos genannte Gott scheint derselbe zu sein, der in Touron im Orontes-Tal unter dem Namen Zeus Koryphaios bis mindestens 396 verehrt wurde: IGLS 652 mit TROMBLEY 1994, 275-278; TROMBLEY 2004, 60-61.

32 Zur Religiosität des Libanios vgl. WIEMER 1995b, 55-57. Einen Überblick über die Kulte und Heiligtümer Syriens in hellenistisch-römischer Zeit vermitteln TEIXIDOR 1989; DENTZER 1989; GAWLIKOWSKI 1989. Speziell zu den auf dem Territorium Antiocheias nachweisbaren Kulten vgl. jetzt den grundlegenden Beitrag von TROMBLEY 2004, bes. 60-66. Die kulturelle Gesamtsituation behandelt MiLLAR 1993, 225ff. 
Dreh- und Angelpunkt der Rede „Für die Tempel“ ist der Appell an den Kaiser als Hüter des Rechts. Andere Gesichtspunkte - das fiskalische Interesse an der landwirtschaftlichen Produktion ${ }^{33}$ und die Aufgabe, die städtische Bausubstanz zu erhalten $^{34}$ - spielen daneben nur eine untergeordnete Rolle. Da am christlichen Bekenntnis des Theodosius nicht zu zweifeln war, kam ein Rekurs auf die Rolle des pontifex maximus, der unter Julian selbstverständlich gewesen war, ${ }^{35}$ natürlich nicht mehr in Frage. Gegenüber Theodosius klammert sich der Heide Libanios an diejenigen Aspekte des spätantiken Kaiserideals, die von Heiden und Christen gleichermaßen akzeptiert werden konnten. Daher weist Libanios dem Kaiser vor allem zwei Aufgaben zu: das Reich gegen äußere Feinde zu schützen und im Inneren für Recht und Ordnung zu sorgen. ${ }^{36}$ In Fragen der Religion kennt die Macht des Kaisers für Libanios keine Grenzen; er gesteht ihm sogar ausdrücklich $\mathrm{zu}$, den öffentlichen Götterkult zu verbieten und den Vollzug des christlichen Kultes unter Androhung der Todesstrafe zu befehlen ( $\$ 52-53$ ):

Du, Kaiser, hättest folgendes Edikt verkünden lassen können: ,Keiner meiner Untertanen soll an die Götter glauben; keiner soll sie ehren oder von ihnen für sich selbst oder für seine Kinder etwas Gutes erbitten, es sei denn schweigend und im verborgenen. Jeder soll den Gott verehren, den ich verehre, und an den ihm geltenden Kulthandlungen teilnehmen; jeder soll die Gebete sprechen, wie man zu ihm betet, und sein Haupt in die Hand dessen legen, der die Gemeinde leitet. Wer nicht gehorcht, muß sterben.' Dieses Edikt hättest du ohne weiteres verkünden lassen können, aber du wolltest den Seelen der Menschen kein Joch auferlegen. ${ }^{37}$

33 Or. 30,10: Die Zerstörung ländlicher Heiligtümer verringert Steuereinnahmen. Der Gedanke, daß Bedrückung der Bauern dem Fiskus schade, begegnet bei Libanios auch in der Rede „Über die Transportdienste“: or. 50,36. Die Sorge um die Beeinträchtigung der Steuereinnahmen soll Arcadius zunächst davon abgehalten haben, die Zerstörung des Marnas-Heiligtums in Gaza zu befehlen: Marc. Diac. v. Porph. 41 (der Passus steht sowohl in der griechischen als auch in der georgischen Version). Die, weswegen Gründen Henri Grégoire (GRÉGOIRE - KUGENER 1930, XLIII-XLIV) diesen Abschnitt für einen späten Zusatz hielt (angebliche Anklänge an Justinians Novelle 103), erweisen sich bei näherer Prüfung als nicht stichhaltig.

34 Städtische Heiligtümer stellen einen ästhetischen Wert dar: or. 30,42 (Tempel sind die „Augen“ der Städte); vgl. or. 30,22-23 (Asklepios-Statue in Beroia); or. 60 (Monodie auf den ApollonTempel in Daphne) mit Nock 1962. Daß solche Erwägungen auch Theodosius oder doch zumindest seinem im Jahre 382 amtierenden (uns leider unbekannten) quaestor sacri palatii nicht völlig fremd waren, zeigt Cod. Theod. 16,10,8 (382, Palladio duci Osdroenae): simulacra ... artis pretio quam divinitate metienda. Zum quaestor sacri palatii vgl. HARRIES 1988 (mit einer Liste der nachweisbaren Amtsinhaber). 383/4 bekleidete übrigens Maternus Cynegius das Amt: or. 49,3 .

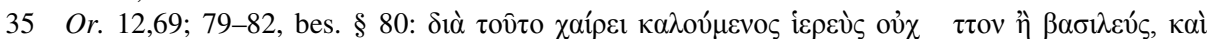

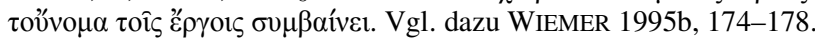

36 Or. 30,14. Dieser Gedanke wird auch in der Rede „Gegen diejenigen, die bei den Statthaltern sitzen" ausgesprochen: or. 51,2-3; vgl. or. 52,2-3.

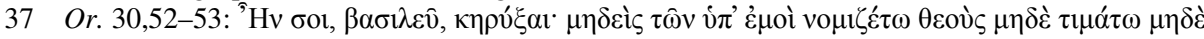

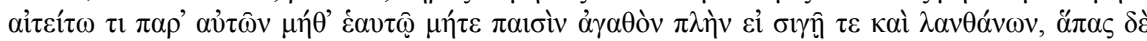

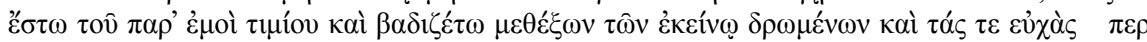

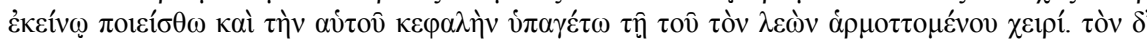


Im religiösen Bereich steht der Kaiser für Libanios also nicht bloß über den Gesetzen, sondern auch über dem Recht: Der Redner behauptet nicht etwa, daß man die Ausübung bestimmter Kulte nicht verbieten dürfe, sondern müht sich vielmehr um den Nachweis, daß es sinnlos und schädlich sei, es zu tun, was bekanntlich zwei kategorial streng zu unterscheidende Aussagen sind. Anders gesagt: Libanios appelliert gegenüber Theodosius keineswegs an ein unverlierbares Recht auf Religionsfreiheit. Sein Anspruch an den Kaiser ist sehr viel bescheidener: Er verlangt von ihm lediglich, dafür zu sorgen, daß der Rechtszustand, den er selbst geschaffen hat, nicht willkürlich verletzt wird. ${ }^{38}$

Dabei setzt der Sophist ohne weiteres voraus, daß die Rechtslage der heidnischen Kulte für das gesamte Reich oder doch wenigstens seinen östlichen Teil genau definierbar ist. Ihre rechtliche Situation hat sich nach Libanios zwar seit Konstantin mehrfach verändert: Auf die Toleranz Konstantins sei unter Constantius ein totales Verbot der heidnischen Kulte gefolgt; im Anschluß an die heidnische Restauration Julians seien die heidnischen Kulte noch kurze Zeit geduldet worden, bis Valentinian und Valens Tieropfer erneut verboten hätten. Diesen Rechtszustand habe Theodosius durch seine Gesetzgebung bestätigt. Im Gegensatz zur modernen Forschung, die mit Recht betont, daß sich die Gesetzgebung der spätrömischen Kaiser jedenfalls vor der Publikation des ,Codex Theodosianus' nicht zu einem kohärenten Normensystem von reichsweiter Geltung fügte, ${ }^{39}$ insistiert also Libanios gerade darauf, daß die Religionsgesetze für einen Reichsteil in seiner Gesamtheit Geltung beanspruchten und sich zu einem in sich stimmigen Regelwerk summierten.

Dieser Befund steht in merkwürdigem Kontrast zu der von Malcolm Errington gemachten Beobachtung, daß die Religionsgesetzgebung des Theodosius in der christlichen Überlieferung ein recht verhaltenes Echo fand ${ }^{40}$ und verlangt nach einer Erklärung. M.E. erklärt sich der ausgeprägte Legalismus der Rede „Für die Tempel“ aus der argumentativen Notlage, in der sich der Heide Libanios gegenüber Theodosius befand. Die Notwendigkeit, den verbliebenen Freiraum für die Ausübung heidnischer Kulthandlungen mit Argumenten zu verteidigen, die auch für Christen annehmbar sind, zwingt den Sophisten dazu, der kaiserlichen Gesetzgebung höchste Bedeutung beizumessen und sie streng nach dem Buchstaben zu interpretieren. Dabei geht er durchgängig von dem Prinzip aus, daß alles erlaubt sei, was nicht ausdrücklich verboten ist, einem Prinzip also, das dem römischen Rechtsdenken durchaus fremd ist: Denn das Rechtssprichwort nulla poena sine lege klingt zwar antik, ist es aber natürlich nicht; es wurde nicht in der

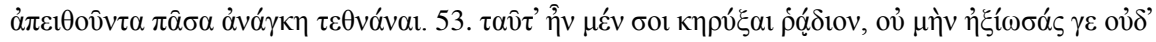

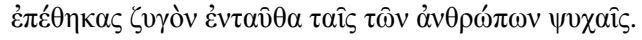

38 Auch sonst fordert Libanios die Einhaltung bestehender Gesetze: or. 33,18; 45,32-33; 48,15; 49,3-7.

39 Vgl. etwa BRINGMANN 1989; auch in: BRINGMANN 2001, 337-347.

40 ERRINGTON 1997a. 
Antike, sondern erst an der Schwelle zum 19. Jahrhundert geprägt. ${ }^{41}$ Der früheste Nachweis findet sich in dem 1801 erschienenen Lehrbuch des in Deutschland gültigen Strafrechts aus der Feder des liberalen Strafrechtsreformers Paul Anselm Feuerbach. ${ }^{42}$ Zwar begegnen bei Juristen severischer Zeit, bei Ulpian und Paulus, vereinzelt Äußerungen, die in diese Richtung gehen $;{ }^{43}$ dem römischen Strafrecht fehlt das Prinzip der Bestimmtheit jedoch durchaus. Die Vermutung liegt nahe, daß diese scheinbare Modernität der Rede „Für die Tempel“ ein Hauptgrund dafür ist, weshalb sie forschungsgeschichtlich eine so große Bedeutung erlangt hat: Man glaubte, es mit einem antiken Zeugen für die Prinzipien der Rechtstaatlichkeit und Religionsfreiheit zu tun zu haben. ${ }^{44}$

Damit Libanios Theodosius als Hüter des Rechts für sich in Anspruch nehmen kann, muß er den Kaiser gegen seinen Präfekten ausspielen. Denn Libanios weiß sehr wohl, daß der Kaiser zwar theoretisch allmächtig, faktisch aber auf Berater und Helfer angewiesen ist. ${ }^{45}$ Der Kaiser kann daher getäuscht und zu Handlungen verleitet werden, die seiner Natur fremd sind; sein Name kann für Handlungen in Anspruch genommen werden, die seinem Willen nicht entsprechen. In unserem Fall sind es eben die bösen Höflinge wie Cynegius, die Theodosius falsch informieren und dadurch veranlassen, Gewalttaten gegen Heiligtümer zu dulden.

Stellt man schließlich die Frage, wer sich bei Libanios der Zerstörung von Tempeln widersetzt, fällt als erstes ins Auge, daß Libanios aktiven Widerstand gegen die Zerstörung von Heiligtümern zwar für die Zukunft vorhersagt, falls der Kaiser ihr weiterhin tatenlos zusehe, für die Vergangenheit und Gegenwart aber mit keinem Wort erwähnt. Diese Feststellung gilt nicht bloß für die Zerstörung des ,großen Heiligtums“, die er staatlichen Instanzen zuschreibt, sondern ebenso für die Angriffe auf ländliche Heiligtümer. Priester müssen der Zerstörung ihrer Heiligtümer hilflos zusehen, Bauern, die mit den Heiligtümern auch Vorräte, Vieh und mitunter sogar ihr Land verlieren, versuchen vergeblich, beim Bischof von Antiocheia Wiedergutmachung für ihre Schäden zu erlangen. Diese Darstellung hat innerhalb der Rede die Funktion, um Sympathie für wehrlose Opfer unprovozierter Gewalttaten zu werben, und ist schon deswegen mit Skepsis zu betrachten. Es konnte nicht in Libanios' Interesse liegen, darauf hinzuweisen, daß

41 Vgl. SCHREIBER 1976, 17; SCHREIBER 1984; WeSEL 1997, 450-452 § 288.

42 FEUERBACH 1801, § 20.

43 Dig. 50,16,131, § 1 (Ulpian); 50,16,244 (Paulus); vgl. dazu LIEBS 1980, 122-123.

44 Bei Jacques Godefroy liegt dieser Zusammenhang auf der Hand: Seine Ausgabe der Rede erschien 1634, während in Deutschland der Dreißigjährige Krieg tobte und wenige Jahre nach dem Fall der Hugenottenhochburg La Rochelle. Ich beabsichtige, dieser Frage an anderem Ort weiter nachzugehen.

45 In ep. 958 ist von einem Brief Theodosius' I. die Rede, der bereits unterzeichnet ist, aber nicht abgeschickt wird. Der Gedanke, daß die Durchsetzung kaiserlicher Anordnungen von den Statthaltern abhänge, taucht in der Korrespondenz des Libanios mehrfach auf: ep. 144; 169; 1201. Zudem weiß Libanios natürlich, daß der Kaiser die Statthalter braucht, weil er nicht überall selbst Recht sprechen kann: or. 51,3. 
die Betroffenen sich mitunter massiv zur Wehr setzten, wie wir aus anderen Quellen wissen. ${ }^{46}$

An keiner Stelle verliert der Sophist auch nur ein Wort darüber, wie sich die Ratsherren Antiocheias zur Zerstörung von Heiligtümern verhielten, obwohl davon auszugehen ist, daß die Ratsherren in ihrer Eigenschaft als Grundherren von der Gewalt gegen ländliche Heiligtümer direkt oder indirekt betroffen waren. Die Erklärung, die Mönche seien nur gegen solche Heiligtümer vorgegangen, die zu Dörfern freier Bauern gehörten, Heiligtümer dagegen, die auf dem Grund und Boden antiochenischer oder apamenischer Ratsherren standen, hätten sie bewußt geschont, ${ }^{47}$ ist schon an sich wenig plausibel. Vor allem aber steht sie im Widerspruch zum Schlußsatz der Rede, in welchem Libanios prognostiziert, daß die „Herren der Äcker“ das Recht gegebenenfalls in ihre eigenen Hände nehmen würden. An dieser Stelle ist offenkundig vorausgesetzt, daß die Gewalt auch Äcker und Dörfer erreichte, die einen städtischen Grundherren hatten. Das beredte Schweigen über die Rolle der Ratsherren verlangt nach einer anderen Erklärung: Libanios will von ihnen nicht sprechen, weil sie die Gewalt gegen die ländlichen Heiligtümer in ihrer Mehrheit entweder billigten oder sich ihr doch zumindest nicht aktiv widersetzen wollten.

\section{Repräsentativität und Realitätsbezug}

Es dürfte auf der Hand liegen, daß wir es bei der Rede „Für die Tempel“ mit einem Zeugnis zu tun haben, dessen historische Aussagekraft in vieler Hinsicht begrenzt ist. Dies gilt zunächst einmal für die zeitliche Dimension: Das Plädoyer des Libanios für die Tempel setzt eine konkrete historische Situation voraus, die nur von kurzer Dauer war. Theodosius beendete die von Libanios beklagte Rechtsunsicherheit nur wenige Jahre, nachdem die Rede „Für die Tempel“ geschrieben worden war, auf ganz andere Art und Weise, als der Sophist sich das gewünscht hatte: Theodosius verbot alle öffentlichen Formen heidnischer Kultausübung: ${ }^{48}$ sein Sohn Arcadius setzte die Todesstrafe auf das Delikt und ordnete die Zerstörung heidnischer Kultstätten auf dem Lande ausdrücklich an. ${ }^{49}$ Diese Gesetze blieben keineswegs toter Buchstabe: Man kam mit der Zerstörung heidnischer Kultbauten in der Diözese Oriens so gut voran, daß Arcadius 397 in einem an den comes Orientis Asterius gerichteten Gesetz anordnen konnte, man solle das ex demolitione templorum stammende Material zum Bau von Wegen, Brü-

46 Soz. h.e. 7,15,12-15 (Tod des Marcellus von Apameia; vgl. Thdt. h.e. 5, 21,5-16); Thdt. h.rel. 17.

47 So aber LIEBESCHUETZ 1972, 68.

48 Cod. Theod. 16,10,10 (391, ad Albinum PUR); 16,10,11 (391, Euagrio PFTO Augustali et Romano COM AEG); 16,10,12 (392, ad Rufinum PPO).

49 Cod. Theod. 16,10,13 (395, Rufino PPO); 16,10,16 (399, ad Eutychianum, PPO). 
cken, Wasserleitungen und Mauern verwenden. ${ }^{50}$ Der heidnische Kult konnte seitdem nicht mehr durch den Appell an das Recht verteidigt werden. Nach 395 konnten Heiden nur noch darauf hoffen, daß man die Religionsgesetze nicht konsequent anwenden würde, und auf einen neuen Julian warten. So kam es, daß die Idolatrie des letzten heidnischen Kaisers, die in der Rede „Für die Tempel“ nur eine marginale Rolle spielt, sich in heidnischen Zirkeln bis ins 6. Jahrhundert hinein hielt. ${ }^{51}$

Auch in räumlicher Hinsicht eignet sich die Rede „Für die Tempel“ nicht für weitreichende Verallgemeinerungen. Der Vagheit seiner Formulierungen zum Trotz hat Libanios offenkundig vor allem die Verhältnisse im nordsyrischen Raum vor Augen, letztlich wohl vor allem diejenigen auf dem Territorium seiner Heimatstadt Antiocheia; Rom und Alexandreia dienen ihm ja nur als Kontrastfolie. Insbesondere hat Libanios zweifellos bewußt darauf verzichtet, die Vorgänge in der Provinz Syria Coele, deren Hauptstadt Antiocheia war, mit paganen Hochburgen zu vergleichen, in denen die städtischen Eliten unter Theodosius I. an ihren altüberkommenen Heiligtümern noch zäh festhielten. Denn da das Netz seiner Kontakte die gesamte Diözese Oriens umspannte, war ihm selbstverständlich bekannt, daß namentlich in Gaza (Palaestina Prima), ${ }^{52}$ in Emesa $^{53}$ und

50 Cod. Theod. 15,1,36 (Asterio COM Orientis, 397): Quoniam vias, pontes, per quos itinera celebrantur, adque aquaeductus, muros quin etiam, iuvari provisis sumptibus oportere signasti, cunctam materiam, quae ordinata dicitur, ex demolitione templorum, memoratis necessitatibus deputari censemus, quo ad perfectionem cuncta perveniant.

51 In der neuplatonischen Schule Athens wurde der Tod des Scholarchen Proklos nach der Regierung Julians datiert: Marin. v. Procli 36. Damascius, der Biograph des athenischen Scholarchen Isidoros, würdigte Julian als Kämpfer gegen das Christentum: V. Isidori 303 Zintzen $=115 \mathrm{~A}$ Athanasssiadi mit HAEHLING 1980.

52 Das Beispiel Gaza ist in letzter Zeit mehrfach eingehend untersucht worden; vgl. etwa DAM 1985; TROMBLEY 1993, I, 188-282 sowie jetzt HAHN 2004, 191-222. Alle Rekonstruktionen bleiben freilich mit der Unsicherheit behaftet, daß die Entstehungs- und Überlieferungsgeschichte der mit Abstand wichtigsten Quelle, der Vita Porphyrii, bislang nicht schlüssig geklärt werden konnte. Die griechische Version der Vita (ediert, ins Französische übersetzt und kommentiert von GRÉGOIRE - KUGENER 1930) scheint auf eine verlorene syrische Vorlage zurückzugehen, von der sich auch eine georgische Version herleitet, die PEETERS 1941 erstmals ediert und zugleich ins Lateinische übersetzt hat. Zwar hat TROMBLEY 1993, I, 246-282 versucht, die griechische Version als ursprünglich zu erweisen, doch ist dieser Versuch als mißglückt zu betrachten: CHILDERS 2001. Weiterhin scheint klar, daß die georgische Version dem verlorenen syrischen Original insgesamt näher steht als die griechische, die durch eine Reihe von Zusätzen und Einschüben erweitert ist. Jedoch scheint Peeters die Skepsis gegenüber dem sachlichen Gehalt der griechischen Vita zu weit getrieben zu haben, zumal sie in den Grundzügen mit der georgischen durchaus übereinstimmt; vgl. jetzt HAHN 2004, bes. 203-204 mit dem wichtigen Hinweis auf die von ESBROECK 1984 erschlossene Homilie des Johannes von Jerusalem (Bischof 386-417), in der Porphyrios als Staurophylax der Kirche von Jerusalem erwähnt wird.

53 Iul. mis. 357C; Thdt. h.e. 3,7,5. Von der positiven Haltung der phönizischen Eliten gegenüber der heidnischen Restauration Julians zeugen auch die in zwei inschriftlichen Kopien erhaltenen, im Namen der dortigen Provinzialversammlung geleisteten vota ob imperium (AE 2000, $1500+$ 1503 = CONTI 2004, 69-72 Nr. 17 + 18), denen DIETZ 2000 eine sehr ausführliche Studie gewidmet hat. 
in Heliopolis-Baalbek (Phoenice), ${ }^{54}$ in Karrhai (Osrhoena) ${ }^{55}$ ja selbst in Apameia, das damals (bis etwa 413) noch zur selben Provinz gehörte wie Antiocheia ${ }^{56}$ noch immer eine wehrhafte Mehrheit hinter den heidnischen Kulten stand.

Für das syrische Antiocheia aber mit seinem riesigen Territorium stimmt das Bild, das sich aus der Rede ergibt, durchaus mit anderen Quellen überein, die uns über die religiöse Situation im letzten Drittel des 4. Jahrhunderts Aufschluß gewähren. Denn auch die epigraphischen und archäologischen Zeugnisse legen den Schluß nahe, daß die Christianisierung dieses Gebietes in den Jahren zwischen 365 und 425 entscheidende Fortschritte machte. ${ }^{57}$ Welche Rolle Gewalt bei diesem Transformationsprozeß spielte, läßt sich mit Hilfe dieser Quellengruppen freilich in der Regel nicht feststellen, und noch weniger erlauben materielle Überreste eine differenzierte Antwort auf die Frage, von wem Gewalttaten verübt wurden. ${ }^{58}$ Ein starkes Argument für die Glaubwürdigkeit des Libanios sehe ich in der Tatsache, daß er die Zerstörung ländlicher Heiligtümer durch Mönche in seiner Rede ja nicht zu beweisen versucht, sondern vielmehr als ein allgemein bekanntes und unstrittiges Faktum behandelt. Zudem wird das gewaltsame Vorgehen gegen heidnische Heiligtümer in den Viten syrischer ,Heiliger' mehrfach rühmend hervorgehoben. ${ }^{59}$ Es stellte also nachweislich einen in syrischen Mönchskreisen lobenswerten Verhaltenstypus dar.

Die Tatsache, daß Theodoret fast zwei Generationen nach Libanios in seiner erbaulichen „Mönchsgeschichte“ das Bild einer friedlichen Infiltration der ländlichen Gebiete Nordsyriens durch meist einzeln auftretende Asketen zeichnet, ${ }^{60}$

54 Vgl. dazu TROMBLEY 1994, II, 154-158.

55 Vgl. dazu unten den Anhang.

56 Vgl. dazu TROMBLEY 1993, I, 122-129; TROMBLEY 1994, II, 283-311.

57 Mit den epigraphischen Zeugnissen hat sich nach der bahnbrechenden Arbeit von LIEBESCHUETZ 1977 (auch in: LIEBESCHUETZ 1990, Nr. VIII) vor allem Frank Trombley eingehend beschäftigt, der 1994 eine ausführliche Untersuchung für ganz Nordsyrien vorlegte (TROMBLEY 1994, II, 247-312). Für das Territorium von Antiocheia liegt nun eine aktualisierte und methodisch verfeinerte Studie aus seiner Feder vor: TROMBLEY 2004, 59-85, in der das epigraphische Material in Form von Tabellen übersichtlich aufbereitet ist.

58 Vgl. dazu WARD-PERKINS in diesem Band.

59 Dies gilt sowohl für die panegyrische Lebensbeschreibung des Rabbula von Edessa (OVERBECK 1865, 169-170; ins Deutsche übersetzt von BICKELL 1874, 175-176) als auch für die noch immer unveröffentlichte Vita des Barsauma von Nisibis: NAU 1913, 272-276; 376-389; NAU 1914, 113-134; 278-289; bes. NAU 1913, 382-385. Auch wenn es sich im Falle des Rabbula tatsächlich lediglich um einen hagiographischen Topos handeln sollte, wie PEETERS 1928, 177 178 (auch in: PEETERS 1951, 145-146) meinte, bliebe die Stelle doch aufschlußreich für die Mentalität des Verfassers und seiner intendierten Leser. Indessen hat nach NAU 1931 nun BoWERSOCK 2000 neue, beachtenswerte Argumente für die Glaubwürdigkeit der Biographie des Rabbulas vorgebracht. Vgl. weiter Thdt. h.rel. 28; Zach. v. Sev. (PO 2, 27ff.).

60 Thdt. h.rel. 4 (Ammianos); 6 (Symeon der Ältere); 14 (Maisymas); 16 (Maro); 17 (Abraames). Wo die gewaltsame Zerstörung eines Heiligtums überhaupt einmal erwähnt wird, erscheint sie als gemeinsame Tat von Dorfbewohnern und einem Asketen: Thdt. h.rel. 28 (Thalaleios); vgl. dazu TROMBLEY 1994, II, 143-173. 
stellt demgegenüber kein Gegenargument von Gewicht dar. Denn Theodoret bot erst recht keine umfassende und ausgewogene Darstellung des Transformationsprozesses, da er seine ,Helden " nach ganz anderen Kriterien auswählte. ${ }^{61}$ Der Bischof von Kyrrhos war zwar keineswegs ein prinzipieller Gegner von Gewalt gegen heidnische Kultstätten; ${ }^{62}$ er betrachtete das Heidentum jedoch als eine in der Gegenwart überwundene Krankheit ${ }^{63}$ und sah die Vorbildlichkeit der von ihm geschilderten Asketen vor allem darin, daß sie die Fesseln der Natur abgestreift und ein engelgleiches Leben geführt hatten. Wir dürfen es Libanios also wohl glauben, wenn er den Mönchen der Provinz Syria Coele eine führende Rolle bei der Zerstörung ländlicher Heiligtümer in seiner Heimatprovinz zuschreibt.

Nun handeln die Mönche bei Libanios zwar mit Rückendeckung durch den antiochenischen Bischof, aber doch auf eigene Faust und Rechnung. Der Bischof Antiocheias erscheint weder als Drahtzieher noch als Anstifter der Gewalt gegen heidnische Heiligtümer. ${ }^{64}$ Diese Passivität ist insofern auffällig, als die moderne Forschung in den Bischöfen die treibende Kraft hinter Übergriffen auf Andersgläubige zu erkennen pflegt ${ }^{65}$ und sich dabei ja auch auf eine Reihe gut dokumentierter Fälle stützen kann. Man könnte daher geneigt sein, Libanios' Darstellung in diesem Punkt für unglaubwürdig zu halten. Für ihre Glaubwürdigkeit spricht indessen das unverdächtige, weil Libanios' Intentionen zuwiderlaufende Detail, daß der Bischof von den Opfern als Schiedsrichter angerufen wird (§ 12). Dies hätten sie wohl kaum getan, wenn Flavianus in dem Ruf gestanden hätte, selbst für die Aktionen der Mönche verantwortlich zu sein. Libanios' Darstellung wird zudem durch die zahlreichen Predigten und Traktate, die Johannes als antiochenischer Presbyter verfaßte, insofern bestätigt, als an keiner Stelle explizit oder auch nur implizit zur Gewaltanwendung gegen heidnische Kultanlagen aufgefordert wird, ${ }^{66}$ und zwar auch dort nicht, wo der Brand des Apollon-Tempels in Daphne, der von Kaiser Julian den Christen zur Last gelegt wurde, ${ }^{67}$ zur Sprache kommt. Johannes deutete den Brand als Beweis der Macht des toten Märtyrers Babylas, dessen Grab Julian hatte verlegen lassen, verzichtete aber darauf, daraus eine Lehre für die Lebenden abzuleiten. ${ }^{68}$ Mitunter ermahnte er seine Gemeinde sogar

61 Zur Absicht der „Mönchsgeschichte“ Theodorets vgl. LEPPIN 1996b.

62 Thdt. h.e. 5,21,6-16 mit LEPPIN 1996a, 171. Vgl. auch Thdt. h.e. 5,29, eine Stelle, auf die Leppin nicht eingeht.

63 Thdt. h.e. 5,21,5; 37,3; affect. 6, 87; Ps. 29, 9 (PG 80, 1069 A-B).

64 Anders, aber m. E. nicht zutreffend LIEBESCHUETZ 1972, 238: ,This line of argument suggests that the activities of the monks were stimulated by the bishop of Antioch“.

65 Vgl. etwa FOWDEN 1978; HAHN 2004, bes. 277-278.

66 Vgl. die nach wie vor unentbehrliche Übersicht bei BAUR 1929, I, 252ff. sowie den thematischen Index in Bd. 13, 2 der leider viel zu wenig benutzten Ausgabe der Mauriner (Paris 1834 $1839^{2}$ ), herausgegeben von Bernard de Montfaucon, s. vv. gentes, gentiles, templa.

67 Iul. mis. 346B; Ammian 22,13,2. Eine von Julian eingesetzte dreiköpfige Untersuchungskommission, der auch Libanios angehörte, vermochte jedoch keinen Schuldigen ausfindig zu machen und sprach mindestens einen Angeklagten ausdrücklich frei: ep. 1376.

68 Jo. Chrys. de S. Babyla 67-113; de S. Babyla Hieromartyre 5-9. Die Zweifel der älteren Forschung, ob der Traktat „Auf den heiligen Babylas und gegen die Heiden“ tatsächlich von Johan- 
ausdrücklich, man solle die Heiden mit Sanftmut und Nachsicht behandeln wie aufmüpfige Kinder. ${ }^{69}$ Da Johannes als Presbyter gehalten war, im Sinne seines Bischofs zu predigen, ${ }^{70}$ dürfen wir diese irenische Haltung gegenüber den Heiden, die in auffallendem Kontrast zur äußerst aggressiven Polemik gegen die Juden steht, ${ }^{71}$ wohl auch für Bischof Flavianus von Antiocheia annehmen, der selbst keine Schriften hinterlassen hat. Da wir zudem wissen, daß der Bischof von Antiocheia über die auf dem Territorium der Stadt ansässigen Mönche keine wirksame Kontrolle auszuüben vermochte, ${ }^{72}$ sollten wir akzeptieren, daß Flavianus kein ,Scharfmacher' vom Schlage eines Marcellus von Apameia oder Porphyrios von Gaza war.

Schließlich läßt sich auch die Tatsache, daß Libanios die Ratsherren in seiner Darstellung bewußt ausblendet, aus den Erfahrungen erklären, die er in seiner Heimatstadt Antiocheia gemacht hatte. Denn die syrische Metropole hatte spätestens seit Konstantin eine soziokulturelle Entwicklung genommen, die den Vorstellungen des Rhetorikprofessors diametral zuwiderlief: Nachdem bereits unter Licinius eine Kirche im Stadtgebiet eingeweiht worden war, ${ }^{73}$ hatte Konstantin auf der Orontes-Insel in der Nähe des Kaiserpalastes eine prächtige Kirche auf oktogonalem Grundriß errichten lassen, die schließlich 341 von seinem Sohn Constantius II. eingeweiht wurde. ${ }^{74}$ Unter Theodosius I. wurde die Stadt von einem Kranz von Märtyrerkirchen gesäumt, deren bedeutendste seit 381 neben dem Leichnam des heiligen Babylas auch denjenigen des Bischofs Meletios beherbergte. ${ }^{75}$ Auch wenn das Stadtzentrum bis zur Jahrhundertwende nur eine einzige Kirche aufzuweisen hatte, ${ }^{76}$ war die christliche Präsenz in Antiocheia seit

nes stammt (verzeichnet bei ALDAMA 1965), hat SCHATKIN 1970 ausgeräumt; ihr wird auch eine kritische Ausgabe des Textes verdankt, die hier zugrundegelegt wird: SCHATKIN 1990.

69 Jo. Chrys. hom. 4,6 in 1. Cor. (PG 61, 38-40); zit. von BAUR 1929, I, 276-277. Theodoret behauptet in seiner „Kirchengeschichte“ (h.e. 5,29) zwar, Johannes habe als Bischof von Konstantinopel die Zerstörung von Heiligtümern in Phönizien betrieben, doch erweist sich diese Aussage im Lichte der Chrysostomos-Korrespondenz als tendenziöse Fiktion; vgl. BAUR 1930, II, 330-331 (mit den Belegen).

70 Zum Verhältnis des Johannes zu Flavianus vgl. BAUR 1929, I, 322-327; TIERSCH 2002, 86ff.

71 Jo. Chrys. hom. 1-8 adv. Iud. (PG 48, 843-942) mit WILKEN 1983; HAHN 2004, 143-145. Eine deutsche Übersetzung findet sich bei BRÄNDLE 1995. Eine in allen Gesamtausgaben fehlende Textpassage edieren BRÄNDLE - HEIMGARTNER - PRADELS 2001.

72 Vgl. dazu HAHN 2004, 152-156.

73 Zur sog. Alten Kirche vgl. BAUR 1929, I, 22-23; ELTESTER 1937, 272ff.; DOWNEY 1961, 336.

74 Zur sog. Neuen Kirche vgl. BAUR 1929, II, 23-24; ElTESTER 1937, 254-260; 267-270; 272278; DOWNEY 1961, 342-349. Die Kirche ist auf dem bekannten Mosaik aus Yakto abgebildet: LASSUS 1934; DOWNEY 1963, Abb. 45-60.

75 Präsentation der Grabungsergebnisse bei LASSUS 1938, 5-44 mit Plan III-IV; vgl. auch LASSUS 1947, 123-128. Die literarischen Zeugnisse hat DOwNEY 1938 zusammengestellt. Vgl. auch ELTESTER 1937, 282-283; DOWNEY 1961, 415-416.

76 Auf die ,Unterversorgung ' der Großstadt Antiocheia mit Pfarrkirchen noch unter Theodosius I. hat einst der protestantische Kirchenhistoriker Walter Bauer in seiner Rezension der monumentalen Chrysostomos-Biographie BAUR 1929-1930 hingewiesen: DLZ 1 (1930), 2210-2212, hier: 2212. 
Konstantin unübersehbar, zumal in der Stadt häufig Konzile abgehalten wurden; besonders unter Constantius II. und Valens versammelten sich in ihr wiederholt Hunderte von Bischöfen aus allen Provinzen der östlichen Reichshälfte. ${ }^{77}$ Libanios freilich hat sie geflissentlich ignoriert - in seiner Rede zum Lobe Antiocheias, die eine detaillierte Beschreibung der Stadtanlage enthält, ${ }^{78}$ bringt er das Kunststück fertig, christliche oder jüdische ${ }^{79}$ Kultbauten mit keinem Wort zu erwähnen. ${ }^{80}$ Vor der Tatsache indessen, daß die städtischen Tempel Antiocheias spätestens seit Constantius II. zunehmend für profane Zwecke genutzt und dadurch ihres Charakters als heidnische Kultstätten entkleidet wurden, konnte auch er nicht völlig die Augen verschließen, wenngleich er sie nur beiläufig erwähnt: Von den vier antiochenischen Tempeln, die Libanios in der Rede „Für die Tempel“ als noch unversehrt bezeichnet, dienten unter Theodosius I. mindestens drei als Räumlichkeiten für profane Aktivitäten; ${ }^{81}$ viele andere Tempel, die Libanios gar nicht (mehr) erwähnt, waren offenbar ganz oder teilweise demoliert worden. ${ }^{82}$

Die Entsakralisierung und Demolierung heidnischer Kultbauten vollzog sich in Antiocheia im wesentlichen gewaltlos - wenn man von der gewaltsamen Zerstörung des Tempels des vergöttlichten Trajan absieht, die von Kaiser Jovian ausgegangen zu sein scheint ${ }^{83}$ - und erfolgte sicher mit Duldung, teilweise wohl auch unter Mitwirkung der antiochenischen Ratsherren, die sich vielleicht schon unter Constantius mehrheitlich zum Christentum bekannten. ${ }^{84}$ Kaiser Julian war tief enttäuscht, daß er bei ihnen nicht mehr Unterstützung für sein Programm einer Wiederherstellung des Götterkultes fand, ${ }^{85}$ das im Süden der Diözese Oriens teilweise enthusiastisch begrüßt wurde. ${ }^{86}$ Die antiochenischen Ratsherren

77 Vgl. dazu etwa BRENNECKE 1988 oder BARNES 1993, der für die Jahre 337 bis 361 auch ein gegenüber SEECK 1919 aktualisiertes Itinerar der Kaiserreisen bietet (218-228).

78 Or. 11,196-264, übersetzt und eingehend kommentiert von MARTIN 1959, $23-61$ sowie bei FATOUROS - KRISCHER 1992.

79 Zur Zeit Theodosius' I. gab es in Antiocheia mindestens zwei Synagogen: DownEY 1961, 447; HAHN 2004, 140-141.

80 Vgl. dazu WIEMER 2003, bes. 463-468.

81 Or. 30,51: Heiligtümer der Tyche, des Zeus (Philios), der Athena und des Dionysos. Das Tycheion Antiocheias war bereits 359 seines Schmuckes beraubt worden (ep. 88,2), der AthenaTempel diente 388 als Versammlungsraum für Anwälte (ep. 847,1), der Dionysos-Tempel im selben Jahr als Gerichtssaal (or. 45,26).

82 Libanios' Liste der Götter, denen Julian in Antiocheia opferte (or. 15,79), ist erheblich länger: Zeus Kasios, Zeus Philios, Hermes, Pan, Demeter, Ares, Kalliope, Apollon; vgl. das Selbstzeugnis Julians (mis. 346B/C): Zeus Philios, Tyche, Demeter, Apollon.

83 Eun. F 29 Blockley = Suda s. v. 'Ioßı $\alpha$ óc; Jo. Ant. F 181 = Excerpta de virtutibus 63 (ebenfalls aus Eunap); vgl. dazu HAHN 2004, 178-180, der betont, daß die Brandstiftung vom Kaiser ausging und von der städtischen Bevölkerung abgelehnt wurde. Freilich bleibt hinter der Episode ein quellenkritisches Fragezeichen, da Eunap sie ohne Zweifel in der Absicht erzählt hat, den christlichen Nachfolger Julians anzuschwärzen.

84 Vgl. dazu WIEMER 1995b, 214-215.

85 Darüber in letzter Zeit ausführlich WIEMER 1995b, 190ff.; HAHN 2004, 163-173.

86 Insbesondere in Phönizien (dazu erschöpfend DIETZ 2000, 807-855), aber offenbar auch in der Provinz Arabia: ILS 9465 = CONTI 2004, 59 Nr. 1. 
verhielten sich mehrheitlich reserviert, wenn es um den Kult der alten Götter ging, und dachten deshalb auch erst gar nicht daran, den Tempel des Apollon in Daphne, dessen Dach unter Julian abgebrannt war, etwa wieder aufbauen zu lassen, sobald Julian einmal tot war. Nach dem Scheitern der heidnischen Restauration Julians machte die Christianisierung der antiochenischen Eliten rasch weitere Fortschritte, und zur Zeit Theodosius' I. drückten nicht wenige Ratsherrensöhne aus christlichen Familien bei Libanios die Schulbank. ${ }^{87}$

Vor diesem Hintergrund nimmt es nicht Wunder, daß Libanios auf die Haltung der antiochenischen Ratsherren zur Gewalt gegen heidnische Kultbauten auf dem Lande nicht eingehen wollte; er wußte nur zu gut, daß die ländlichen Tempel für die meisten von ihnen keinen Wert (mehr) darstellten, für den es sich zu kämpfen lohnte. Diese Isolierung des Libanios von den antiochenischen Ratsherren, die auch in anderen Texten der theodosianischen Zeit deutlich zu erkennen ist,$^{88}$ macht zum einen verständlich, weshalb der Sophist alle Hoffnung auf den Kaiser setzte, und läßt zum anderen ermessen, wie verdüstert sein Lebensabend gewesen sein muß. Die aktuelle Forschung betont, daß die Gewalt gegen heidnische Heiligtümer nicht primär von den Kaisern ausging, ${ }^{89}$ und aus der Sicht des modernen Historikers hat es damit auch seine Richtigkeit. Einem Heiden wie Libanios hätte diese Erkenntnis indessen nur geringen Trost gespendet. Er, der alle Hoffnungen auf den Kaiser als Hüter des Rechts gesetzt hatte, mußte noch erleben, daß Theodosius den heidnischen Kulten jeglichen Rechtsschutz entzog.

\section{Anhang}

Der argumentierende Teil der Rede „Für die Tempel“ endet mit einer wütenden Attacke auf den christlichen Prätorianerpräfekten Maternus Cynegius, die von einem konkreten Vorfall, der Zerstörung eines großen Heiligtums an der Reichsgrenze, ihren Ausgang nimmt (§ 43-44). Zu welcher Stadt dieses Heiligtum gehörte, ist seit dem 17. Jahrhundert umstritten. Der Calvinist und gelehrte Kommentator des ,Codex Theodosianus', Jacques Godefroy $(\dagger 1652),{ }^{90}$ sprach sich für Edessa, die Hauptstadt der Provinz Osrhoene, ${ }^{91}$ aus; der Jansenist Sébastien Lenain de Tillemont $(\dagger 1698)$, der Verfasser der „Histoire des Empereurs“ dagegen, das von Libanios gemeinte Heiligtum in Karrhai (Harran) lokalisieren

87 Vgl. das von PETIT 1957, 95-135 zusammengestellte Material.

88 Besonders deutlich ist diese Entfremdung in der Rede „An die, die nicht reden wollen“ (or. 35) und in den beiden Reden über den Stadtrat (or. $48+49$ ).

89 Vgl. etwa LEPPIN 2003, 124-126; 169-181 oder HAHN in diesem Band.

90 Zur Person vgl. Dufour 1991.

91 GODEFROY 1743, 301: Nam etsi Aedessae nominatim ibi non meminerit (sc. Libanius), neque templum Aedessenum nominet, non magis quam hic; attamen non indubie id indicat, cum magnificentiam eius pluribus commendat, \& finibus Persarum vicinum fuisse ait, refertumque innumeris simulacris ... Ihm folgt REISKE 1793 in seiner Ausgabe z. St.

92 Zur Person vgl. NEveu 1966. 
zu können. ${ }^{93}$ Seither schwankt die Forschung zwischen diesen beiden Positionen. ${ }^{94}$

Ich bin zu der Überzeugung gekommen, daß Godefroy das Richtige traf. Für die Lokalisierung in Edessa sprechen zum einen sachliche Gründe: Edessa war seit dem Perserfrieden Jovians die wichtigste Grenzfestung in der Region und hatte am Ende des 4. Jahrhunderts eine starke christliche Gemeinde. ${ }^{95}$ Karrhai dagegen blieb bis in islamische Zeit hinein ein Refugium heidnischer Religionsausübung; der Bote, der den Karrhenern die Nachricht vom Tode Julians überbrachte, soll gesteinigt worden sein. ${ }^{96}$ Der große Tempel des Mondgottes, in welchem Julian vor Beginn des Perserfeldzuges geopfert hatte, ${ }^{97}$ bestand bis zum Jahr $1035 \mathrm{n}$. Chr. ${ }^{98}$ Zum anderen aber erlaubt die Lokalisierung des großen Heiligtums in Edessa zu erklären, weshalb Libanios weniger den ästhetischen Wert als den militärischen Nutzen des Heiligtums betont; solange das Heiligtum aufrecht stand, habe jeder Angriff auf die Stadt scheitern müssen, weil die Umfassungsmauer des Heiligtums jedem Ansturm getrotzt habe (§ 44). Libanios reagiert hier offenkundig, was man bisher nicht gesehen hat, auf die Legende vom Briefwechsel zwischen Jesus und dem edessenischen König Abgar. ${ }^{99}$ Denn zur Zeit des Theodosius war der Brief, den Jesus angeblich an Abgar geschrieben hatte, bereits um den Zusatz erweitert, daß kein Feind jemals über Edessa herrschen solle. Während Eusebios ${ }^{100}$ und sein Übersetzer Rufinus ${ }^{101}$ den Brief in einer Fassung präsentieren, die diesen Zusatz noch nicht enthält, ${ }^{102}$ war diese Version der Abgar-Legende zur Zeit, als Libanios schrieb, zumindest in Edessa nachweislich bereits höchst aktuell: Als die fromme Pilgerin Egeria im April 384 nach Edessa kam, erzählte ihr der Bischof persönlich, daß seit den Tagen des Herrn noch jeder feindliche Angriff auf Edessa mit Hilfe dieses Briefes zurückgeschlagen worden

93 Tillemont 1720, 232-233.

94 GREEN 1992, 52 bezieht Libanios' Darstellung, die sie jedoch offenkundig nur aus zweiter Hand kennt, wieder auf Harran, ebenso TROMBLEY 1994, II, 139 Anm. 30. HAHN 2004, 286-287 Anm. 46 bezieht die Stelle hingegen ohne Begründung auf Edessa.

95 Vgl. KiRSTEN 1959; KIRSTEN 1963; SEGAL 1970.

96 Or. 17,35; Zos. 3,34,2.

97 Or. 18,214; 298; Ammian. 23,3,2; Thdt. h.e. 3,26,2-3.

98 CRAMER 1986, bes. 642; 648-649.

99 Es ist weder möglich noch nötig, an dieser Stelle auf die Entstehung und Entwicklung der Abgar-Legende näher einzugehen, zumal diese zuletzt von MAREK 2004 (mit umfangreichen Literaturhinweisen und einer überraschenden These - Abgar IX. sei zum Christentum übergetreten und habe die eusebianische Korrespondenz archivieren lassen) eingehend behandelt worden ist. Eine neue Ausgabe der „Doctrina Addai“, basierend auf der vollständigeren St. Petersburger Handschrift, bietet HOWARD 1981.

100 Eus. h.e. 1,13,10.

101 Rufin. h.e. $1,13,10$.

102 Bereits Prokopios (bell. pers. 2,12,26) hegte Zweifel an der Authentizität dieses Zusatzes, weil ihn die zeitgenössischen Schriftsteller nicht kannten, und Egeria selbst weist ausdrücklich darauf hin, daß die Version der Korrespondenz, die man ihr in Edessa zeigte, „vollständiger“ (vere amplius) gewesen sei als diejenige, die sie aus ihrer Heimat kannte: Itin. Egeriae 19,18-19. 
sei. ${ }^{103}$ Es ist dieser Anspruch, gegen den Libanios polemisiert, wenn er die Uneinnehmbarkeit Edessas einem Tempel zuschreibt.

\section{Bibliographie}

Aldama, J.A. de, Repertorium pseudochrysostomicum (Documents, études et repertoires publiès par l'Institute de Recherche et d'Histoire des Textes 10), Paris 1965.

Arce, J.J., Construcciones de templos paganos en epoca del emperador Juliano (361-363 d.C.), RSA 5 (1975), 201-215.

Barnes, T.D., Constantine and Eusebius, Cambridge, MA - London 1981.

Barnes, T.D., Constantine's Prohibition of Pagan Sacrifice, AJPh 105 (1984), 69-72.

Barnes, T.D., Athanasius and Constantius: Theology and Politics in the Constantinian Empire, Cambridge, MA - London 1993.

Barnes, T.D., Statistics and the Conversion of the Roman Aristocracy, JRS 85 (1995), $135-147$.

Baur, C., Johannes Chrysostomus und seine Zeit, 2 Bde., München 1929-1930.

Baynes, N.H., Constantine the Great and the Christian Church, PBA 15 (1931), 341-442 [ND Oxford 1972].

Bickell, G., Ausgewählte Schriften der syrischen Kirchenväter Aphraates, Rabulas und Isaak von Ninive, Kempten 1874.

Bleicken, J., Zum Regierungsstil des römischen Kaisers. Eine Antwort auf Fergus Millar, Frankfurt am Main 1982.

Bowersock, G.W., The Syriac Life of Rabbula and Syrian Hellenism, in: T. Hägg - P. Rousseau (Hrsg.), Greek Biography and Panegyric in Late Antiquity, Berkeley u. a. 2000, 255-272.

Bradbury, S., Constantine and the Problem of Anti-Pagan Legislation in the Fourth Century, CPh 89 (1994), 120-139.

Brändle, R. - Jegher-Brucher, V. (Hrsg.), Johannes Chrysostomus, Acht Reden gegen die Juden, Stuttgart 1995.

Brändle, R. - Heimgartner, M. - Pradels, W., Das bisher vermisste Textstück in Johannes Chrysostomus, Adversus Iudaeos, Oratio 2, ZAC 5 (2001), 23-49.

Brennecke, H.C., Studien zur Geschichte der Homöer. Der Osten bis zum Ende der homöischen Reichskirche (Beiträge zur historischen Theologie 73), Tübingen 1988.

Bringmann, K., Tradition und Neuerung in der Gesetzgebung christlicher Kaiser des 4. Jhdts. gegen Heiden, Häretiker und Juden, in: Mehl, A. - Schneider, W.C. (Hrsg.), Festschrift für Lothar Graf zu Dohna, Darmstadt 1989, 13-28.

Bringmann, K., Ausgewählte Schriften, Frankfurt am Main 2001.

Brown, P.R.L., St. Augustine's Attitude to Religious Coercion, JRS 54 (1964), 107-116.

Brown, P.R.L., Religion and Society in the Age of Saint Augustin, London 1977.

103 Itin. Egeriae 19,2-17; bes. 13: Nam et postmodum quotienscumque voluerunt venire et expugnare hanc civitatem hostes, haec epistola prolata est et lecta est in porta, et statim nutu Dei expulsi sunt omnes hostes. 
Brown, P.R.L., Power and Persuasion in Antiquitiy: Towards a Christian Empire, Madison, Wisc. 1992.

Childers, W., The Georgian Life of Porphyry of Gaza, StudPatr 35 (2001), 374-384.

Conti, S., Die Inschriften Kaiser Julians (Altertumswissenschaftliches Kolloquium 10), Stuttgart 2004.

Cramer, W., Harran, in: RAC 13 (1986), 634-650.

Dagron, G., Naissance d'une capitale. Constantinople et ses institutions de 330 à 451 (Bibliothèque byzantine, Etudes 7), Paris 1974.

Dam, R. van, From Paganism to Christianity in Late Antique Gaza, Viator 16 (1985), 120.

Dentzer, J.-M., Le sanctuaire syrien, in: Dentzer, J.-M. - Orthmann, W. (Hrsg.), Archéologie et histoire de la Syrie II. La Syrie de l'époque achémenide à l'avènement de l'Islam, Saarbrücken 1989, 297-322.

Dietz, K., Kaiser Julian in Phönizien, Chiron 30 (2000), 807-855.

Downey, G., The Shrines of St. Babylas at Antioch and Daphne, in: Stillwell, R. (Hrsg.), Antioch-on-the-Orontes, Bd. II: The Excavations of 1933-1936, Princeton 1938, 4548.

Downey, G., A History of Antioch in Syria from Seleucus to the Arab Conquest, Princeton 1961.

Downey, G., Ancient Antioch, Princeton 1963.

Dufour, A. - Schmidlin, B. (Hrsg.), Jacques Godefroy (1587-1652) et l'humanisme juridique à Genève, Basel 1991.

Eltester, W., Die Kirchen Antiocheias im 4. Jh., ZNW 36 (1937), 251-286.

Errington, R.M., Constantine and the Pagans, GRBS 29 (1988), 309-318.

Errington, R.M., The Accession of Theodosius the Great, Klio 78 (1996), 438-453.

Errington, R.M., Christian Accounts of the Religious Legislation of Theodosius I, Klio 79 (1997a), 398-443.

Errington, R.M., Church an State in the First Years of Theodosius I, Chiron 27 (1997b), 21-72.

Esbroeck, M. van, Jean II de Jérusalem et les cultes de S. Étienne, de la Sainte-Sion et de la croix, AB 102 (1984), 99-134.

Fatouros, G. - Krischer, T. (Hrsg.), Libanios, Antiochikos (or. XI). Zur heidnischen Renaissance in der Spätantike, Wien - Berlin 1992.

Feuerbach, P.A., Lehrbuch des gemeinen in Deutschland gültigen Rechts, Gießen 1801.

Förster, R. (Hrsg.), Libanius. Opera, Bd. III, Leipzig 1906.

Fowden, G., Bishops and Temples in the Eastern Roman Empire, JThS n.s. 29 (1978), 5378.

Fowden, G., Rez. F. Trombley, Hellenic Religion und Christianisation, JRS 85 (1995), 341-344.

Gawlikowski, M., Les temples dans la Syrie à l'époque hellénistique et romaine, in: Dentzer, J.-M. - Orthmann, W. (Hrsg.), Archéologie et histoire de la Syrie II. La Syrie de l'époque achémenide à l'avènement de l'Islam, Saarbrücken 1989, 323-346.

Godefroy, J. (Hrsg.), Libanii Antiocheni pro templis gentilium non exscindendis, ad Theodosium M. Imp. Oratio: ante MCCC ferme annos conscripta, Genf 1634.

Godefroy, J., Opera iuridica minora, Lyon 1733.

Godefroy, J. (Hrsg.), Codex Theodosianus cum perpetuis commentariis Iacobi Gothofredi, Bd. VI,1, Leipzig 1743. 
Green, T.M., The City of the Moon God: Religious Traditions of Harran (Religions in the Graeco-Roman World 114), Leiden 1992.

Grégoire, H. - Kugener, M.-A. (Hrsg.), Marc le Diacre. Vie de Porphyre, évêque de Gaza, Paris 1930.

Haehling, R. von, Die Religionszugehörigkeit der hohen Amtsträger des Römischen Reiches seit Constantins I. Alleinherrschaft bis zum Ende der Theodosianischen Dynastie (324-450 bzw. 455 n. Chr.) (Antiquitas, 3. Reihe, Bd. 23), Bonn 1978.

Haehling, R. von, Damascius und die heidnische Opposition im 5. Jahrhundert n. Chr., JbAC 23 (1980), 82-95.

Hahn, J., Gewalt und religiöser Konflikt. Studien zu den Auseinandersetzungen zwischen Christen, Heiden und Juden im Osten des Römischen Reiches (von Konstantin bis Theodosius II.) (Klio-Beihefte n. F. 8), Berlin 2004.

Harries, J., The Roman Imperial Quaestor from Constantine to Theodosius II, JRS 78 (1988), 148-172.

Howard, G. (Hrsg.), The Doctrine of Addai, Chico 1981.

Jarry, J., Inscriptions arabes, syriaques et grecques du Massif du Bélus en Syrie du Nord, Annales islamologiques 7 (1967), 139-220.

Kirsten, E., Edessa, in: RAC 4 (1959), 552-598.

Kirsten, E., Edessa, eine römische Grenzstadt des 4. bis 6. Jh. im Orient, JbAC 6 (1963), 144-172.

Kloft, H., Liberalitas principis. Herkunft und Bedeutung. Studien zur Prinzipatsideologie (Kölner historische Abhandlungen 18), Köln - Wien 1970.

Kraft, H., Kaiser Konstantins religiöse Entwicklung (Beiträge zur historischen Theologie 20), Tübingen 1955.

Lassus, J., La mosaïque de Yakto, in: Elderkin, G.W. (Hrsg.), Antioch-on-the-Orontes, Bd. I: The Excavations of 1932, Princeton 1934, 114-156.

Lassus, J., L'église cruciforme de Kaoussié, in: Stillwell, R. (Hrsg.), Antioch-on-theOrontes, Bd. II: The Excavations of 1933-1936, Princeton u.a. 1938, 5-44.

Lassus, J., Sanctuaires chrétiens de Syrie. Essai sur la genèse, la forme et l'usage liturgique des édifices du culte chrétien, en Syrie, du IIIe siècle à la conquête musulmane (Institut français d'archéologie de Beyrouth. Bibliothèque archéologique et historique 42), Paris 1947.

Lenski, N., Failure of Empire: Valens and the Roman State in the Fourth Century A.D., Berkeley 2002.

Leppin, H., Von Constantin dem Großen zu Theodosius II. Das christliche Kaisertum bei den Kirchenhistorikern Socrates, Sozomenus und Theodoret (Hypomnemata. Untersuchungen zur Antike und ihrem Nachleben 110), Göttingen 1996a.

Leppin, H., Zum kirchenpolitischen Kontext von Theodorets Mönchsgeschichte, Klio 78 (1996b), 212-230.

Leppin, H., Constantius und die Heiden, Athenaeum n.s. 87 (1999), 457-480.

Leppin, H., Theodosius der Große, Darmstadt 2003.

Liebeschuetz, J.H.W.G., Antioch: City and Imperial Administration in the Later Roman Empire, Oxford 1972.

Liebeschuetz, J.H.W.G., Epigraphic Evidence on the Christianisation of Syria, in: Fitz, J. (Hrsg.), Akten des XI. Internationalen Limeskongresses (Székesfehérvár 30.8.6.9.1976), Budapest 1977, 485-508.

Liebeschuetz, J.H.W.G., From Diocletian to the Arab Conquest, Great Yarmouth - Norfolk 1990. 
Liebs, D., Alexander Severus und das Strafrecht, in: Bonner Historia-Augusta-Colloquium 1977/78, Bonn 1980, 115-147.

Lizzi, R., La politica religiosa di Teodosio I. Miti storiografici e realtà storica, RAL 9. ser. 7 (1996), 323-361.

Loy, R. van, Le „Pro Templis“ de Libanius, Byzantion 8 (1933), 7-38; 389-404.

Malosse, P.-L., Rhétorique et psychologie antiques: le portrait de Julien dans l'oeuvre de Libanios, Ktema 20 (1995), 319-338.

Marek, C., Jesus und Abgar. Das Rätsel vom Beginn einer Legende, in: Fuhrer, T. - Michel, P. - Stotz, P. (Hrsg.), Geschichten und ihre Geschichte, Basel 2004, 269-310.

Martin, R., Antioche décrite par Libanius, in: Festugière, A.-J., Antioche païenne et chrétienne. Libanius, Chrysostome et les moines de Syrie (Bibliothèque des Écoles Françaises d'Athènes et de Rome 194), Paris 1959, 23-61.

Millar, F., The Emperor in the Roman World (31 BC - AD 337), London ${ }^{2} 1992$ (1. Aufl. 1977).

Millar, F., The Roman Near East 31 BC - AD 337, Cambridge, MA - London 1993.

Nau, F., Résumé de monographies syriaques: Histoire de Barsauma de Nisibe, Revue de l'Orient chrétien 18 (1913), 272-276; 376-389.

Nau, F., Résumé de monographies syriaques: Histoire de Barsauma de Nisibe, Revue de l'Orient chrétien 19 (1914), 113-134, 278-289.

Nau, F., Les „,belles actions“ de Mar Rabboula, évêque d'Edesse de 412 au 7 août 435 (ou 436), Revue de l'histoire de religions 52 (1931), 97-135.

Neveu, B., Un historien à l'École de Port Royale. Sébastien Le Nain de Tillemont 16371698, Den Haag 1966.

Nock, A.D., Sapor I and the Apollo of Bryaxis, AJA 66 (1962), 307-310.

Norman, A.F., Libanius, Selected Works, with an English Translation, Introduction and Notes, Bd. II: Selected Orations, London 1977.

Overbeck, J.J. (Hrsg.), Ephraemi Syri, Rabbulae episcopi Edesseni, Balaei aliorumque opera selecta, Oxford 1865.

Peeters, P., La vie de Rabboula, évêque d'Èdesse ( $† 7$ août 436), Recherches de sciences religieuses 18 (1928), 170-204.

Peeters, P., La vie géorgienne de Saint Porphyre de Gaza, AB 65 (1941), 65-216.

Peeters, P., Recherches d'histoire et de philolologie orientales (Subsidia hagiographica 27), Bd. I, Brüssel 1951.

Petit, P., Libanius et la vie municipale à Antioche au IVe siècle après J.-C. (Institut français d'archéologie de Beyrouth. Bibliothèque archéologique et historique 62) Paris 1955.

Petit, P., Les étudiants de Libanius. Un professeur et ses élèves au Bas Empire (Études prosopographiques 1), Paris 1957.

Petit, P., L'empereur Julien vue par le sophiste Libanios, in: Braun, R. - Richer, J. (Hrsg.), L'empereur Julien. De l'histoire à la legende (331-1715), Bd. I, Paris 1978, 67-87.

Price, S.R.F., Rituals and Power: The Roman Imperial Cult in Asia Minor, Cambridge 1984.

Reiske, J.J. (Hrsg.), Libanius sophista. Orationes et declamationes, Bd. III, Altenburg 1793.

Romano, R., Libanio. In difesa dei templi. Introduzione, traduzione e note (Quaderni di Koinonia 7), Neapel 1982. 
Schatkin, M., The Authenticity of St. John Chrysostom's De Sancto Babyla, Contra Iulianum et Gentiles, in: Granfield, P. - Jungmann, J.A. (Hrsg.), Kyriakon. Festschrift Johannes Quasten, Bd. I, Münster 1970, 474-489.

Schatkin, M. (Hrsg.), Jean Chrysostome, Discours sur Babylas. Introduction, texte critique, traduction et notes par M. Schatkin avec la collaboration de C. Blanc et B. Grillet suivi de Homélie sur Babylas. Introduction, texte critique, traduction et notes par B. Grillet et J.-N. Guinot (Sources chrétiennes 362), Paris 1990.

Schreiber, H.-L., Nulla poena sine lege, in: Handwörterbuch der deutschen Rechtsgeschichte 3 (1984), 1103-1111.

Schreiber, H.-L., Gesetz und Richter - Zur geschichtlichen Entwicklung des Satzes nullum crimen nulla poena sine lege, Frankfurt am Main 1976.

Seeck, O., Regesten der Kaiser und Päpste für die Jahre 311 bis 476 n. Chr. Vorarbeit zu einer Prosopographie der christlichen Kaiserzeit, Stuttgart 1919.

Segal, J.B., Edessa, the Blessed City, Oxford 1970.

Straub, J.A., Vom Herrscherideal in der Spätantike (Forschungen zur Kirchen- und Geistesgeschichte 18), Stuttgart 1939.

Teixidor, J., Sur quelques aspects de la vie religieuse dans la Syrie à l'époque hellénistique et romaine, in: Dentzer, J.-M. - Orthmann, W. (Hrsg.), Archéologie et histoire de la Syrie II. La Syrie de l'époque achémenide à l'avènement de l'Islam, Saarbrücken 1989, 81-95.

Tiersch, C., Johannes Chrysostomus in Konstantinopel (397-404). Weltsicht und Wirken eines Bischofs in der Hauptstadt des oströmischen Reiches (Studien und Texte zu Antike und Christentum 6), Tübingen 2002.

Tillemont, S. Lenain de, Histoire des empereurs et des autres princes qui ont regné durant les six prémiers siécles, Bd. V, Paris 1720.

Trombley, F., Hellenic Religion and Christianization c. 370-529 (Religions in the GraecoRoman World 115), 2 Bde., Leiden 1993-1994.

Trombley, F., Christian Demography in the Territorium of Antioch (4th - 5th c.): Observations on the Epigraphy, in: Sandwell, I. - Huskinson, J. (Hrsg.), Culture and Society in Later Roman Antioch, Oxford 2004, 59-85.

Wallace-Hadrill, A., The Emperor and His Virtues, Historia 30 (1981), 298-319.

Wesel, U., Geschichte des Rechts. Von den Frühformen bis zum Vertrag von Maastricht, München 1997.

Wickert, L., Princeps (civitatis), in: RE XXII (1954), 1998-2296.

Wiemer, H.-U., Libanius on Constantine, CQ n.s. 44 (1994), 511-524.

Wiemer, H.-U., Die Rangstellung des Sophisten Libanios unter den Kaisern Julian, Valens und Theodosius. Mit einem Anhang über Abfassung und Verbreitung von Libanios' Rede „Für die Tempel“, Chiron 25 (1995a), 89-130.

Wiemer, H.-U., Libanios und Julian. Studien zum Verhältnis von Rhetorik und Politik im vierten Jahrhundert n. Chr. (Vestigia. Beiträge zur Alten Geschichte 46), München $1995 b$.

Wiemer, H.-U., Vergangenheit und Gegenwart im „Antiochikos“ des Libanios, Klio 85 (2003), 442-268.

Wiemer, H.-U. (Hrsg.), Staatlichkeit und politisches Handeln in der römischen Kaiserzeit (Millennium-Studien 10), Berlin 2006.

Wilken, R.L., John Chrysostom and the Jews: Rhetoric and Reality in the Late 4th Century, Berkeley u. a. 1983. 
Wytzes, J., Libanius et les lois, in: Boer, M.B. de - Edridge, T.A. (Hrsg.), Hommages à Maarten J. Vermaseren, Bd. III, Leiden 1978, 1334-1350. 



\title{
The End of the Temples: An Archaeological Problem
}

\author{
BRYAN WARD-PERKINS
}

In this article I wish to explore a simple question - "Why does archaeological evidence not play a more central role in the increasingly sophisticated and nuanced literature on the end of Roman paganism?"1 Historians are well aware of the danger that our Christian texts may over-dramatize the end of the traditional cults, with accounts of saints like Martin in Gaul, John Chrysostom in Syria, and Shenoute in Egypt rampaging through cities and countryside forcibly destroying temples and smashing idols. ${ }^{2}$ They are also aware that the legislation in the Theodosian Code, ordering the closure and sometimes the demolition of the temples, is both contradictory and impossible to judge as to its effectiveness. There are even occasions where a textual reference to the 'destruction' of a temple can be tested against the material record, and be found to be greatly exaggerated. Justinian, for instance, is said by Procopius to have "pulled down the temples" at Philae; but the surviving remains of the buildings prove beyond doubt that their structures were hardly touched. ${ }^{3}$ Why then does the archaeological evidence, which is both ideologically neutral (unlike any of our texts) and scattered evenly across the empire, not play a greater part in assessing the reliability of texts, and the extent to which their messages can be generalized? ${ }^{4}$

There should, in theory, be plenty of archaeological data for the problem we are investigating, by contrast, for instance, with the earlier pagan assault on Christianity. This attack had primarily been aimed against people rather than buildings, and such assaults are extremely difficult to document archaeologically - Christian martyrdom, for instance, only shows up clearly in the material record because Christians, after the triumph of the Church, were able to build great basilicas over

1 I am very grateful for help in the preparation of this article from Jitse Dijkstra and Georgios Deligiannakis. I would also like to thank Johannes Hahn and his colleagues for inviting me to a colloquium in Münster that was both highly instructive and very enjoyable.

2 For instance, CASEAU 2004, 120: "All these stories are to be taken with extreme caution, however. The word destruction might cover mere symbolic gestures such as knocking over a statue from its pedestal, as well as the complete dismantling of the building."

3 Prok. bell. Pers. 1.19.37 (trans. DEWING 1914, 188-189); DiJKSTRA 2004, 152-154 (with reference to the earlier literature).

4 See, for example, the writings of Béatrice Caseau, who is very knowledgeable about the available archaeological evidence and uses it intelligently, but who none the less relies much more heavily on texts: CASEAU 2001 and 2004. 
the graves of their martyrs. Pre-Constantinian Christianity was a religion of rooms adapted for worship within ordinary houses, like those excavated at Dura Europos, and of tiny monuments, like the first shrine of St. Peter on the Vatican Hill. ${ }^{5}$ These humble structures are found archaeologically only in exceptional circumstances - the frescoes of the house-church at Dura Europos were preserved because most of the house was sealed under a defensive earthwork, and the shrine on the Vatican would never have been identified as an important Christian memorial, if Constantine had not placed his high altar directly over it. Unsurprisingly, given the humble nature of early Christian monuments, the pagan persecutions were aimed much less at these material objects than they were at Christians themselves.

Paganism, on the other hand, had enjoyed centuries - in Egypt, indeed, millennia - of prosperity and of establishment as the official religion of the state. As a result, the Roman empire was filled with an extraordinary wealth of temples, and of statues and reliefs of the gods. Furthermore these statues were often of stone, which is extremely durable, and the temples (except in the most remote northern provinces) were always of solid stone or brick, and sometimes on a massive scale (as at Baalbek or at Egyptian Thebes). The fate of some religions is difficult to trace archaeologically - pre-Constantinian Christianity is one (except from graves with inscriptions); Germanic paganism is another. Egyptian and Graeco-Roman paganisms, however, are almost perfect for the archaeologist their temples and statues are amongst the most impressive and solid remains of their age.

The Christian challenge to paganism was very explicitly a challenge to these imposing pagan buildings and sacred images, rather than a persecution of individuals, and the triumph of Christianity was marked by the destruction or removal of cult objects, above all statues, and by the destruction or, at least, closure of the temples. These are changes that should, in theory, show up in the archaeological record. Archaeology can only tell us indirectly about the history of living people, but it is very good at telling us the history of things, and archaeologists are highly fortunate when those things are as solid and difficult to miss as the remains of ancient paganism. Why then is the archaeological contribution to our knowledge of the end of Roman paganism so patchy and so marginal to the story as it is currently told?

Of course, there are some pagan objects, even from the Roman period, that do not show up in the archaeological record. For instance, the chopping down of a sacred grove can never be detected in the surviving material record - unless ancillary buildings and altars leave signs of its fate. Similarly, cult objects in wood very seldom survive, and it is hard to imagine their removal or destruction leaving any tangible trace. The destruction of images in precious metal is equally invisible, since their remains will have ended up in the melting-pot. When Constantine 
stripped pagan temples of their gold and silver cult statues, Eusebius tells us that the metal was then purified "by smelting with fire" - in other words, it was melted down and added to the imperial coffers. ${ }^{6}$ This action probably did leave a significant material trace, in the issuing of a new and abundant gold coinage under Constantine; but sadly we will never be able to trace the ounces of gold in these solidi back to particular desecrated pagan shrines.

However, the pagan temples leave impressive archaeological remains, and pagan statues of marble or stone, whether shattered or intact, should also survive well in the soil. ${ }^{7}$ Why, then, have we learned comparatively little from the archaeological record about what happened to these in the face of a triumphant Christianity? In part, we must certainly blame the priorities of archaeologists themselves. As is well known, until recently (particularly in the Mediterranean region), there was simply no interest in documenting how classical buildings and classical objects fell out of use. 'Excavations' were really massive clearance exercises down to the first impressive classical remains, and even quite substantial things that stood in the way could be swept aside without adequate record. The Palatine in Rome, for instance, was cleared down to imperial levels in the late nineteenth and earlier twentieth centuries. Somewhere on this hill was the important early-medieval oratory, and later monastery, of S. Cesario in Palatio; but, besides two fragmentary frescoes and a few scattered architectural elements, nothing was found of it, and its precise location remains in doubt. ${ }^{8}$ Ecclesiastical buildings in Rome, even from the early Middle Ages, were always solid structures, and the monastery must also have had its own graveyard - that S. Cesario and its monks disappeared without trace under the pick-axes of the excavators of the Palatine tells us a great deal about pre-modern excavation methods and priorities.

Unfortunately, huge numbers of temples, probably by far the majority of those known today, were uncovered early in the history of archaeology - because they were prime targets for archaeologists interested in fine sculpture and impressive architecture. At Luna in Italy, for instance, both of the temples so far identified were the subject of nineteenth-century excavation, and were also the initial focus of attention when the systematic uncovering of the city began in the 1970s, before the archaeologists working there had mastered the techniques of stratigraphic excavation. Effectively, the remains of these two buildings were simply cleared of the overburden of soil. ${ }^{9}$ If there ever were traces in the archaeological record of when and how these temples closed, they have gone for ever; the only chance now of shedding any light on the chronology and the process of abandonment would be to find and identify architectural elements from them, reused in later datable buildings.

6 Eus. v.C. 3.54 .

7 Though there is, of course, the certainty that many a marble statue was burnt for lime.

8 AUGENTI 1996, 50-55 and 64.

9 Frova 1973, cols. 2-19. 
Sadly, a similar story can be told of hundreds of temples, including some about whose fate we would very much like to know more. When John Wood began excavations at Ephesus in 1869, on behalf of the British Museum, he started, not unnaturally, on the site which he (correctly) identified as that of the great temple of Diana of the Ephesians. What Wood found was that the temple had been very effectively demolished - remarkably little of it remained in situ and the haul of sculpture to take back to his pay-masters was extremely disappointing. He also identified the foundations of what he believed to be a church built over the site. ${ }^{10}$ We would dearly love to know whether this temple - to the many-breasted goddess who earned the opprobrium of St Paul - was the victim of early and systematic Christian demolition, and perhaps of the construction of a triumphalist church on the site. But, because of the nature of Wood's clearance, all such evidence has gone, and it is not even certain that his 'church' was really a Christian building. ${ }^{11}$

Neglect by archaeologists of what we might term 'abandonment history', has certainly also played a major part in depriving us of information about the end of pagan statuary. In a recent book, Eberhard Sauer, has drawn attention to this, outlining the evidence for deliberate attacks on some pagan sculpture, and pointing out correctly that archaeologists have traditionally been very much keener to stick arms and heads back on statues, than to worry about how they ever became detached. ${ }^{12}$ Although a very great deal of evidence will have been lost, for instance to the restorers of the eighteenth century, Sauer is also certainly correct that it is not too late to submit some long-excavated finds and standing monuments to this kind of analysis - though, of course, it will often be impossible to tell whether a headless Venus lost her head to a deliberate blow, or to accidental damage when subsidence or an earthquake toppled her from her plinth. We should, however, note that - in order to place the evidence for deliberate damage to statues in its true perspective - we would also need to investigate seriously the evidence that other pagan sculpture was never deliberately harmed, and consider the reasons behind the preservation within the cities of Late Antiquity of some undamaged pagan imagery. ${ }^{13}$ Again, as with the broken statues, archaeologists in the past have tended to fall under the thrall of the objects themselves, and have spent too little time wondering how and why they managed to survive.

Sauer offers, as one example of the kind of research that could fruitfully be done, the temples of Egypt, many of which (but not all) were carefully worked over, at tremendous effort, to destroy elements of their sacred sculpture (fig. 1).

10 WoOD 1877; the evidence for the church is presented on the plan at p. 262.

11 See Foss 1979, 86-87.

12 SAUER 2003, esp. pp. 17-18.

13 As argued by LEPELLEY 1993, 13. Lepelley compares the saving of pagan statues from destruction in Late Antiquity, to the work of Alexandre Lenoir salvaging royal tombs and other monuments of French history during the Revolution - which is an interesting parallel and thoughtprovoking, but perhaps a little over-romantic. 
The phenomenon is well known, but, as far as I am aware, has never been studied systematically. There appear to be characteristics common to all the sites where this defacing occurred - above all, an attack on the carved human figures, but no interest in the sacred hieroglyphic texts and only occasional attacks on the representations of animals (despite the fact that these are generally sacred, and often, indeed, gods in their non-human form). On the relief illustrated here, for instance, the sacred vulture is spared, but the enemies of Egypt (in the grasp of the pharaoh), who are entirely neutral in religious terms, have been carefully defaced. All of this points to a considerable degree of ignorance about the ancient religion when the work was carried out. On the other hand, there are also interesting differences between what happened at various temples. At Medinet Habu (the mortuary temple of Rameses III near Thebes) the defacing can perhaps be associated with the building of a Christian church within the inner courtyard of the temple, but elsewhere, as at Dendera, no church was ever established within the building. Yet at Dendera the work of defacing was even more systematic - often the whole figure was attacked, whereas at Medinet Habu effort was concentrated on the face and hands. ${ }^{14}$

If a lack of scholarly interest in how temples and cult statues were abandoned were the only cause of problems, we would at least have a clear path forwards, with the potential for greatly improving the archaeological data forthwith. Unfortunately, this is not the only difficulty - there are also substantial innate problems with the archaeological record. Archaeology is very good at illustrating broad trends, the end of paganism and the Christianization of the empire being no exception: in the archaeological record the temples are adapted to new uses or torn down for their building materials, and a rash of new churches appears. But, if we seek tight chronology and the human motivation behind this change, we will generally seek them in vain. For reasons that will become clear, there is little chance that archaeological evidence will ever produce a significantly refined chronology of when temples were closed; or provide conclusive evidence as to whether the process was essentially violent, or pacific. Although we would like to know whether paganism went out with a bang, or died with a whimper, I doubt whether even the best archaeological work will ever be able to tell us.

All too often, even when perfectly excavated, the remains of a temple are not sufficiently well preserved to give us reliable evidence of its abandonment history. In the case of cities that survived into the sixth and seventh centuries, as most Roman cities did, the abandoned temples became a major source of cheap stone, and were, over time, systematically taken apart, down to the level of their foundations, or even below them. In the process, any trace of their immediate post-abandonment history will have been destroyed, and, with it, any accurate indication of when and how they were abandoned. For instance, in the 1980s,

14 SAUER (2003, figs. 47-54) illustrates several examples at Dendera. His fig. 50 (at p. 94) is particularly interesting: all the human figures are defaced, but the god Horus, in the form of a falcon, is left intact, down to the detail of his prominent erect penis. 
careful excavation revealed the location and plan of the Capitolium of Verona, facing out over the forum of the city. ${ }^{15}$ A statue-base datable to $379 / 82$ happens to survive from this same city, which records the re-erection of a statue "in the crowded forum, after it had lain for a long time in the Capitolium", which suggests that the temple was already in decay by the end of the 370 s. ${ }^{16}$ It would be very nice if the archaeological evidence could confirm or deny this impression. However, what were found in the 1980s were badly robbed-out foundations that could give no hint as to the building's earlier fate.

Unfortunately, the chronological precision that we need, in order to chart the demise of paganism, is generally too tight for even well-preserved archaeological evidence to be of a great deal of use - it makes a considerable difference to our full understanding of the phenomenon, whether a temple was abandoned in 350 , in 400 , or in 450 . If closed in 350 , the temple had gone even before the emperor Julian's 'pagan revival'; if in 400, it may have been forcibly closed as a result of Theodosius I's anti-pagan legislation; but if in 450, it may have been quietly left to decay, because no-one any longer cared for it. Even the best excavation can only very rarely offer such chronological precision. Charting archaeologically the abandonment of a building's original function requires clear and datable traces of the change of use to survive in the soil, whereas all too often the evidence is either absent or ambiguous. Temples, in common with all classical public buildings, had solid stone and marble floors and might well have been kept clean, even if their religious use had ended and they had been adapted to a new function. Only when the roof fell in, or an accumulation of rubbish was allowed to build up - or, better still, when another solid building was built over the site - will any firm indication of abandonment have accrued. In researching this paper, I had a look at a number of Oxford churches that have recently been converted to secular use (two have become the libraries of colleges, and one a café) - in all of these cases the structural alterations have been minimal, and it would be very hard, or impossible, to detect the change of use archaeologically, in any normal circumstances.

At Aphrodisias in Caria, the site of the great temple of Aphrodite was converted during Late Antiquity into the cathedral of the city, dedicated to the Mother of God. The initial clearance of the site by archaeologists provided no date for this transformation; however, more recent (and much more patient) work has suggested, on the basis of pottery evidence, a date in the second half of the fifth century for the construction of the church. ${ }^{17}$ This is a major step forward in our understanding of the abandonment history of the temple. But it cannot tell us whether the bishop of Aphrodisias took over a semi-ruinous building, a structure that was already being used for some other function, or a temple which was in good condition and still revered by many within the city.

15 CAVALIERI MANASSE 1987, 15-17.

16 CIL V 3332.

17 SMITH - RATTÉ 1995, 44-46. 
Our best hope for archaeological precision, both as regards chronology and motivation, is undoubtedly violent destruction, particularly by fire, since this can leave material evidence that is both unequivocal and datable. Such evidence should exist, since several texts from the East record the burning of temples (whose wooden roofs made them vulnerable to arson). ${ }^{18}$ Most famously, this was how the great temple of Zeus Marnas at Gaza was eventually destroyed, according to the account given in the Life of Bishop Porphyry. ${ }^{19}$

There are indeed a few archaeologically attested cases of temples destroyed by fire, at roughly the right date for us to attribute the destruction, with reasonable confidence, to Christians. For instance, the floor of a rural sanctuary of Minerva at Breno (in Italy's Val Camonica), excavated in 1986, was found to be covered in a layer of charcoal and ash (datable to after the fourth century), which also contained a marble statue of the goddess, missing its head and arms. ${ }^{20}$ Other complicated scenarios could be devised to explain this damage, but the simplest explanation is that this was deliberate destruction by Christians. Similarly, at Cyrene (in modern Libya) the vast marble cult statue of the temple of Zeus was broken into small pieces, and there are marks of burning on the limestone walls of the cella. Here there is no evidence to date the damage, since the temple was crudely excavated in 1861 and 1926. As a result, the case is not as strong as that at Breno - but Christian activity is, none the less, the most likely explanation. ${ }^{21}$

What is surprising, however, given the textual references, is that there are not more such cases. It is, indeed, tempting to deduce from this that temples were very seldom burnt down. However, although I suspect this is true - particularly in towns, where it can never have been safe to start major fires - I do not think it can yet be demonstrated archaeologically with any confidence. Too many temples were, after their closure, robbed down to their floor-level of their stone-work, for us to say with any confidence how they were initially destroyed - whether through neglect, or through violent damage, even if the latter was by fire. In the case of Gaza's Marneion, for instance, our text tells us that, subsequent to the fire, the remains of the temple were demolished and a church built on the site. ${ }^{22}$ Activity on this scale is quite likely to have removed all archaeological trace of the original fire, however dramatic and destructive. It may be only at rural shrines, such as that at Breno, which were then entirely abandoned, that we will find good, undisturbed evidence of the initial cause of abandonment.

18 The evidence is presented by CASEAU 2001, 93.

19 Marc. Diac. v. Porph. 69. For the text and its context: HAHN 2004, $202 \mathrm{ff}$.

20 CANTino Wataghin 1997, 133-135, describing also a similar find at Industria. As far as I am aware, the Breno site has never been fully published, but a good illustrated interim report by ROSSI (1986) appeared in Soprintendenza Archeologica della Lombardia, Notiziario 1986, 6567.

21 GOODCHILD - REYNOLDS 1958, 39-40.

22 Marc. Diac. v. Porph. 75-79. 
As Richard Bayliss has recently pointed out, the archaeological evidence for Christian damage to temples is all too seldom clear-cut, and too often open to wishful thinking - as I also discovered when I tracked a number of supposed cases back to their original publications. ${ }^{23}$ Most disappointing was the evidence from the Mithraeum under the church of Santa Prisca in Rome, whose excavators believed they had found clear signs of its violent destruction by Christians, and which is cited by Sauer as a particularly good example of passionate religious iconoclasm. ${ }^{24}$ The cult-niche, with its stucco figures, was certainly badly damaged when discovered, and bits of the relief were found scattered around the room; many of the frescoes too were badly damaged. But a careful examination of the published photographs of the latter did not suggest to me that they had been savagely and systematically attacked with axes, as their excavators claimed; rather, the plaster looks to have been in a generally very poor state when uncovered, and to have decayed randomly across the wall. Even some frescoed heads, which should have been the first target of iconoclasts, were well preserved when excavated, including the haloed head of Mithras himself (which, we are told in the published report, was destroyed, not by fourth-century Christians, but by a botched attempt at restoration in 1953). ${ }^{25}$ As for the stucco figures in the niche stucco is a fragile medium, and, while they might have been deliberately damaged, it also seems possible that they had decayed and fallen apart. The head of Mithras, although detached from its original setting, was found in very good condition - a Christian iconoclast could easily have crushed it under foot.

A rather different example - this time where the problem of interpretation is primarily due to a lack of firm dating evidence - is the Gallo-Roman sanctuary and villa excavated at Saint-Aubin-sur-Mer on the coast of Normandy. Here the limestone statue of a seated Celtic goddess, broken into pieces, was discovered

23 BAYLISS 2004, 23-25. Bayliss discusses the thorny question of whether one can tell Christian destruction from that wrought by earthquakes, foreign invaders, or neglect. For earthquakes and temples, see Rothaus 2000, 39-44 and 60-61 - but see also the review (and subsequent debate) in the on-line Bryn Mawr Classical Review 2002.02.25. The case of Corinth is interesting: the excavations were good by earlier twentieth-century Mediterranean standards; but, even in this case, we cannot confidently say what brought the pagan buildings down.

24 VERMASEREN - VAN ESSEN 1965, 129-131, 151 and 241f. SAUER 2003, 134-136.

25 See VERMASEREN - VAN ESSEN 1965, plates 53-65. The excavators too were puzzled at the survival of some of the sacred representations, though this did not deflect them from their overall interpretation: "After the destruction of the cult-niche and some of the monuments (though not others, the real meaning of which was not understood), the whole building was filled with rubbish" (VERMASEREN - VAN ESSEN 1965, 241-242). SAUER (2003, 135-136) argues - at some length - that it was precisely because of their 'passionate hate' that the Christian iconoclasts wielded their axes so inaccurately! The head of Mithras, which, according to Sauer, was a particular focus of attack, is at the right-hand end of his fig. 63 (on p. 135). VERMASEREN and VAN ESSEN $(1965,150)$ recount its true (and more banal) fate: "Of the head [of Mithras], only the outline has been preserved, with part of the halo; the rest of the face was lifted off in 1953 by the Istituto Centrale del Restauro, but did not respond to treatment and is now destroyed." 
dumped into a half-filled well (fig. 2). ${ }^{26}$ Unfortunately, this find was made more than sixty years ago in very difficult circumstances - during a rescue-excavation in 1942-1944, necessitated by the building of the Wehrmacht's Atlantic Wall. Violent Christian destruction (perhaps in the late fourth century) is an entirely plausible explanation for the find; but there are some problems with this interpretation. The statue was certainly not destroyed at the same time as the temple, which, oddly enough, had been demolished long before the well in which the goddess was found had even been dug. Furthermore, no datable finds from the fill of the well were recorded, so when exactly it was filled, and the statue dumped, cannot now be known for certain. The event may have occurred as the result of violent Christian destruction; but it is just possible that it happened much earlier, or much later, long after paganism was a contested issue. The statue may have been discarded, less as an act of hot-blooded iconoclasm, than as part of a process of 'tidying up' by local inhabitants, now somewhat embarrassed by their outmoded pagan statuary. Should we imagine a snatch-squad of monks, descending on the site and smashing the statue to pieces, before hurling it into an abandoned well, or should we envisage a Gallo-Roman owner, quietly ordering a superfluous object to be broken up and dumped into a convenient hole?

It can certainly be difficult to tell, from archaeological evidence alone, quite how hot-blooded an act of destruction was. In the case of buildings, our sources tell us that some temples were demolished by zealous Christians, in order to end their pagan use - but, archaeologically, it would be very difficult to identify destruction of this kind, from the 'calm' demolition of an already abandoned structure, in order to recycle its stone.$^{27}$ In both cases, the building materials will have been carted off for reuse.

In the destruction of some sculpture, there does seem to be evidence of gratuitous violence, suggesting that this was fuelled by religious fervour. Sauer illustrates and discusses two marble statues, of Serapis and Cybele, from Sarsina in Italy, which were broken into hundreds of pieces (over three hundred in the case of the Serapis). ${ }^{28}$ It is hard to imagine how this could have happened, unless religious enthusiasm powered the sledge hammer. On the other hand, even systematic destruction need not always be attributed to hatred or enthusiasm. At some point in Late Antiquity, probably in the fifth century, the shrine of the imperial cult at Aphrodisias, the Sebasteion, was transformed into an area of shops. When this happened, the sculpture that decorated the monument, though much of it was set high above the ground, was systematically worked over. The figures of emperors, and of personified provinces, were left untouched, except for the removal of the genitals on nude figures; but representations of the pagan gods and god-

26 EBLÉ 1948, 365-383. The statue was published by BÉQUIGNON 1949, 83-97.

27 The recorded cases of systematic destruction are collected by CASEAU 2001, 92. See also BAYLISS 2004, 22f.

28 SAUER 2003, 139-142. 
desses were carefully chiselled out. ${ }^{29}$ In a case like this, should we imagine an angry bishop ordering the violent destruction of pagan images, or the civic authorities calmly and systematically up-dating a monument for new times? As so often, both are serious possibilities, leaving it open to the present-day observer to read into the evidence the scenario they prefer. Such are the problems with the archaeological evidence; but such also are the fascinating possibilities that it raises.

\section{Bibliography}

Augenti, A., Il Palatino nel Medioevo: Archeologia e topografia (secoli VI-XII), Rome 1996.

Bayliss, R., Provincial Cilicia and the Archaeology of Temple Conversion, Oxford 2004.

Béquignon, Y., Statue gauloise découverte à Saint-Aubin-sur-Mer (Calvados), MMAI 43 (1949), 83-97.

Cantino Wataghin, G., La conversion de l'espace: quelques remarques sur l'établissement materiel chrétien aux IV ${ }^{\mathrm{e}}-\mathrm{V}^{\mathrm{e}}$ siècles, d'après l'example de l'Italie du Nord, in: Rouche, M. (ed.), Clovis: histoire et mémoire. Le baptême de Clovis, l'événement, Paris 1997, 127-139.

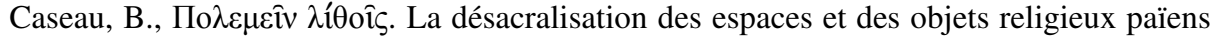
durant l'Antiquité tardive, in: M. Kaplan (ed.), Le sacré et son inscription dans l'espace à Byzance et en Occident (Byzantina Sorbonensia 18), Paris 2001, 61-123.

Caseau, B., The Fate of Rural Temples in Late Antiquity and the Christianisation of the Countryside, in: Bowden, W. - Lavan, L. - Machado, C. (eds.), Recent Research on the Late Antique Countryside (Late Antique Archaeology 2), Leiden - Boston 2004, 105-144.

Cavalieri Manasse, G. (ed.), Il Veneto nell'età romana, vol. 2: Note di urbanistica e di archeologia del territorio, Verona 1987.

Dewing, H.B., Procopius, Works, vol. 1 (Loeb Classical Library), London - Cambridge, MA 1914.

Dijkstra, J.H.F., A Cult of Isis after Justinian? ZPE 146 (2004), 137-154.

Eblé, E., Découvertes à Saint-Aubin-sur-Mer, Gallia 6 (1948), 365-383.

Foss, C., Ephesus after Antiquity: A Late Antique, Byzantine and Turkish City, Cambridge 1979.

Frova, A. (ed.), Scavi di Luni. Relazione preliminare delle campagne di scavo 1970-1971, Rome 1973.

Goodchild, R.G. - Reynolds, J.M., The Temple of Zeus at Cyrene, PBSR 13 (1958), 3062.

29 SмIтH 1987, 97-98. While the iconoclasts failed to identify a few of the obscurer gods (e.g. Hygieia), and hence spared them, they were certainly better-informed than the people who chiselled out the reliefs at Medinet Habu and Dendera: all the emperors and provinces were spared, and almost all the gods were destroyed. 
Hahn, J., Gewalt und religiöser Konflikt. Studien zu den Auseinandersetzungen zwischen Christen, Heiden und Juden im Osten des Römischen Reiches (von Konstantin bis Theodosius II.) (Klio-Beih. n. F. 8), Berlin 2004.

Kraeling, C.H., The Christian Building (= vol. 8, part 2 of The Excavations at DuraEuropos, Final Report), New Haven 1967.

Lepelley, C., Le musée des statues divines. La volonté de sauvegarder le patrimoine artistique païen à l'époque théodosienne, CArch 41 (1993), 5-15.

Rossi, F., [Interim report on excavations at Breno], Notiziario de Soprintendenza Archeologica della Lombardia 1986, 65-67.

Rothaus, R.M., Corinth, the First City of Greece: An Urban History of Late Antique Cult and Religion (Religions in the Graeco-Roman World 139), Leiden 2000.

Sauer, E., The Archaeology of Religious Hatred in the Roman and Early Medieval World, Stroud 2003.

Smith, R.R.R., The Imperial Reliefs from the Sebasteion at Aphrodisias, JRS 77 (1987), 88-138.

Smith, R.R.R. - Ratté, C., Archaeological Research at Aphrodisias in Caria 1993, AJA 99 (1995), 33-58.

Toynbee, J. - Ward Perkins, J., The Shrine of St. Peter and the Vatican Excavations, London - New York - Toronto 1956.

Vermaseren, M.J. - van Essen, C.C., The Excavations in the Mithraeum of the Church of Santa Prisca in Rome, Leiden 1965.

Wood, J.T., Discoveries at Ephesus, Including the Site and Remains of the Great Temple of Diana, London 1877. 


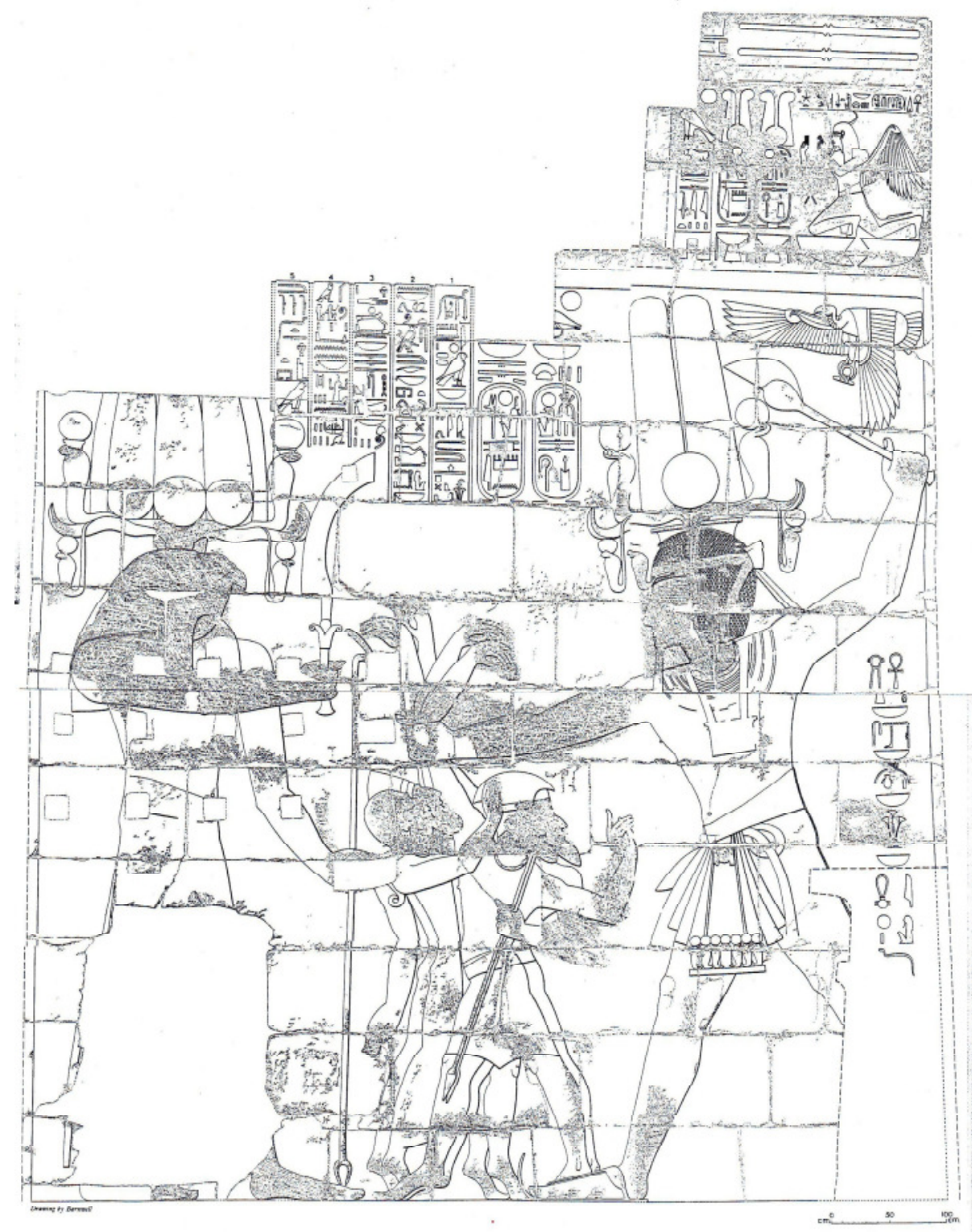

Fig. 1: Medinet Habu, mortuary temple of Rameses III: defaced relief 


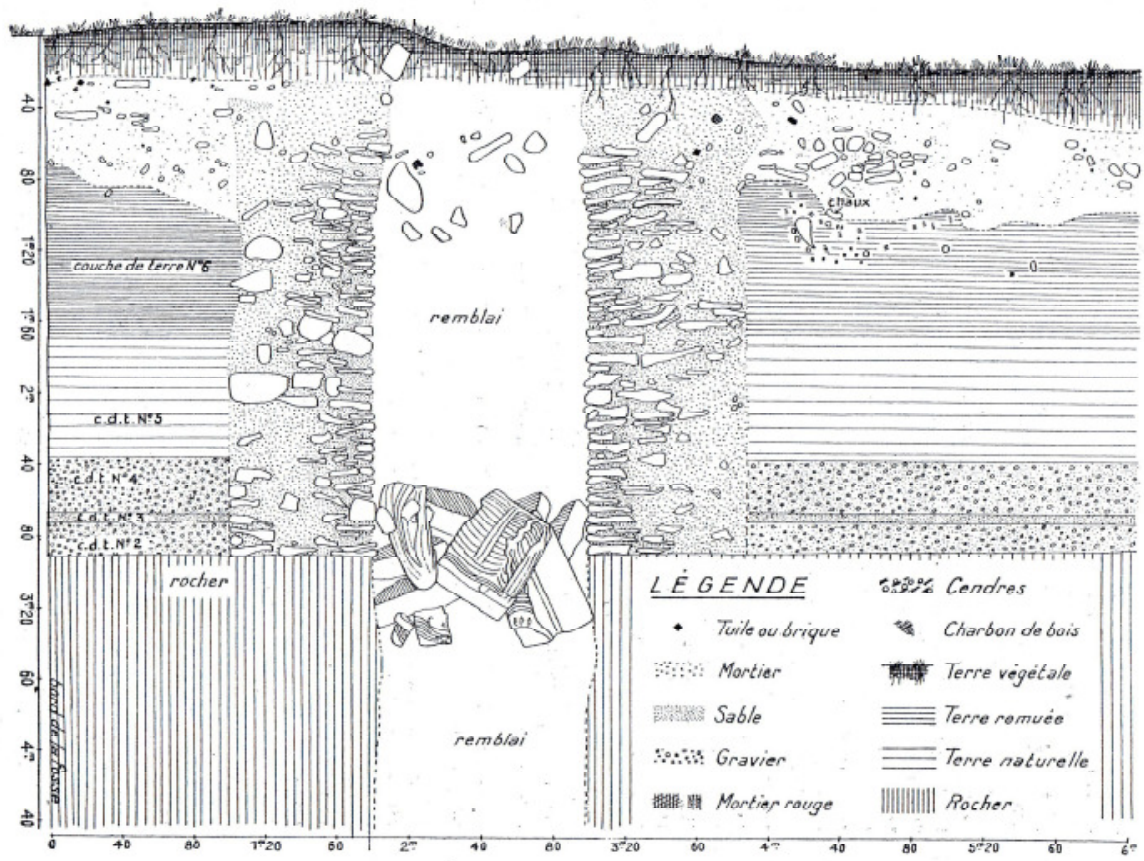

Fig.2: Saint-Aubin-sur-Mer: broken statue of Celtic goddess dumped into a well 



\title{
Gesetze als Waffe? Die kaiserliche Religionspolitik und die Zerstörung der Tempel
}

\author{
JOHANNES HAHN
}

Im oberägyptischen Panopolis, $1.700 \mathrm{~km}$ von der Reichshauptstadt entfernt, spricht ein Asket vor einer Menschenmenge über die Märtyrerverehrung: dies vielleicht just zur Stunde einer der zahllosen Arbeitssitzungen jener JuristenKommission, die in Konstantinopel im Auftrag des Kaisers Theodosius II. an der Kompilation des Gesetzeswerkes arbeitet, das dann am 15. Februar 438 als Codex Theodosianus veröffentlicht werden sollte. Im Verlaufe seiner Predigt ruft er seinen Zuhörern, einfachen christlichen Fellachen, in Erinnerung, wie Herrschaft und Macht auch in ihrem Leben allenthalben erfahrbar sind. Die Präsenz von Kaiser und Imperium veranschaulicht er am Wirken der Gesetze, genauer - denn hierauf bezieht er sich - der kaiserlichen Religionsgesetzgebung.

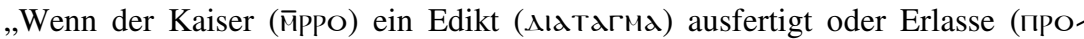

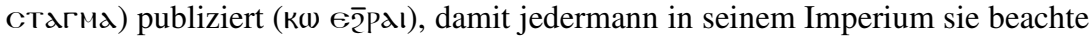
und befolge, so geschieht das mit großer Befehlsgewalt und Machtbefugnis: hin in alle Länder des Reiches, in alle Städte und Dörfer, ob nah oder fern. Und es herrscht dann große Furcht in seinem ganzen Reich, dass jeder auch das, was der Herrscher befohlen hat, mit großer Sorgfalt ausführe - von den Präfekten (єпархос) und Generälen, von den Comites (кюмєC) und Provinzstatthaltern $(\overline{2} \mathrm{H}$ гем(1) bis hin zu den Tribunen und Protoi und den Soldaten, dass sie achtgeben und bereitstehen bei ihren Einheiten (Tdzlc); die Duces (АOYzl) und Provinzstatthalter aber, dass sie tätig werden in den ihnen unterstellten Verwaltungssprengeln (€парx€la), und das ausführen, was der Herrscher ihnen geboten hat, und sie daher nun denjenigen, der sich dem Edikt des Kaisers widersetzt, zum Tode verurteilen mit der Machtbefugnis und dem Schwert des Herrschers, ohne jede Rücksicht. Denn der Kaiser schont weder Präfekt noch General noch Provinzstatthalter, auch nicht die Tribunen und die Protoi und auch nicht die Soldaten und ebenso wenig die Zivilisten (паганос), jegliche Art von Volk nicht, sei es Bischof oder Priester oder Mönch. Vielmehr pflegen die Genannten das Geheiß des Herrschers an einem jeden zu exekutieren, auf den die Bestimmungen der kaiserlichen Erlasse zutreffen. “1
\end{abstract}

1 É. AmÉlineau (ed.), Oeuvres de Schenoudi, t. II, fasc. 3, Paris 1914, p. 523,7-22; beachte auch die (besser edierte) Parallelüberlieferung: C. WESSELY (ed.), Griechische und koptische Texte theologischen Inhalts I. Studien zur Palaeographie und Papyruskunde 9, Leipzig 1909, 159-161 
Im Umland der oberägyptischen Provinzstadt, 60 Tagesreisen vom Kaiserhof entfernt, erleben einfache Provinziale das Imperium als Dominat: als ein absolutes Herrschaftssystem, in dem der ferne Kaiser kraft seiner Gesetze, Edikte und Reskripte - ausgeführt durch eine hierarchisch gegliederte, loyale und effiziente Beamtenschaft, diese sekundiert vom Militär - seinen Ratschluss und Willen durchsetzt, im besonderen seinen Untertanen auch religiöse Normen aufzuzwingen vermag. Die Vorstellung jener einfachen Nilbauern von ihrem Kaiser unterscheidet sich damit kaum von jenem Bild, das die Gesetze des Theodosius II. oder seiner Vorgänger in ihrer Auseinandersetzung mit Häretikern, Heiden etc. selbst vermitteln wollen: uneingeschränkte Autorität und unbedingter Gestaltungswillen des Kaisers gewinnen unmittelbare Geltung und Umsetzung in Administration und Gesellschaft.

Doch mögen sogar jenen koptischen Fellachen in ihrer bescheidenen Lebenswelt gelegentlich Zweifel an diesem idealisierten Bild gekommen sein: Eigentums- und Gewaltdelikte vermochte die kaiserliche Verwaltung nach dem beredten Zeugnis der Papyri jedenfalls nicht zu unterbinden und noch weniger zu sanktionieren. Und selbst im religiösen Feld agierte jedenfalls ein Klostervorsteher wie Schenute, der, wie eben aufgezeigt, so eindringlich über die Kaisermacht zu predigen wusste, ungeachtet genauer Kenntnis der juristischen Lage nahezu unverhohlen gegen gültige Gesetze; in Antinoupolis musste er sich so auch vor dem Statthaltergericht, bei dem Klagen gegen ihn eingereicht wurden, gegen massive Vorwürfe zur Wehr setzen. ${ }^{2}$

Welche genaue Bedeutung besaß nun die kaiserliche Religionspolitik, präziser: die Gesetze und Erlasse im 4. und beginnenden 5. Jahrhundert, und dies im besonderen für das Schicksal der Tempel? Diese Frage bedeutet natürlich zunächst: Welchen Zeugniswert können die im Codex Theodosianus und zwar im Buch 16, Kapitel 10 unter dem Titel De paganis, sacrificiis et templis bewahrten, ja hier sogar schon bequem in chronologischer Abfolge zusammengestellten Texte beanspruchen? Bietet diese Serie von Texten, immerhin unsere grundlegende Quelle für die anti-pagane Gesetzgebung und Politik von Konstantin bis Theodo-

(= Nr. 48a-c). Hier zitiert (mit geringfügigen Änderungen) die Übersetzung des Passus von HORN 1986, 16-19; ebd. 5 zur Datierung des Textes.

$2 \mathrm{Zu}$ den gerichtlich erhobenen Vorwürfen - die im Kontext Schenutes anti-paganer Aktionen insbesondere auch den für ihn gefährlichen Vorwurf der Anstiftung zur öffentlichen Unruhe umfassten - siehe zuletzt EMMEL 2008, 162ff. mit Vorstellung und Diskussion der Quellenstellen, weiterhin auch BEHLMER 1993. Aufschlussreich für die ,Interpretation' der Gesetzeslage durch Schenute ist seine Erklärung zur Plünderung und Zerstörung von Kultstatuen aus dem Hause seines Intimfeindes Gesios - ein früherer, in Panopolis als honoratus ansässiger praeses der Thebaïs -, bei der er formuliert: „Wenn es illegal sein soll, Götterbilder aus dem Hause dieses Mannes zu entfernen, ist es dann auch illegal, sie aus ihren Tempeln zu entfernen? Denn die Gegenstände, wegen derer die gerechten Herrscher in ihrer Liebe zu Gott Anweisungen erteilt haben, die Tempel zu zerstören und bis zu den Fundamenten niederzureißen oder sie mitsamt ihrer Götterbilder zu zerschlagen - es sind diese (selben) Objekte, die wir von diesem Ort (= dem Privathaus des Gesios) entfernt haben.“ („Let our Eyes“, Frgm. 2 § 8f. = ZJ 27-28, ed. EMMEL 2008, hier 197). 
sius II., ein getreues Abbild der Entwicklung der spätantiken Religions- und Tempelgesetzgebung dieser Zeit? ${ }^{3}$ Wichtiger noch, spiegeln diese von den Juristen des jüngeren Theodosius kompilierten Gesetze und Erlasse zugleich die historische Realität und die Dynamik des kaiserlichen Vorgehens gegen Kulte und Kultbauten wider? Lassen die hier formulierten programmatischen Aussagen zugleich den Schluss auf entsprechende Exekution im ganzen Imperium zu? Diese Fragen, die zugleich eine schlüsselhafte Bedeutung für das fundamentale Phänomen der Geschichte des spätantiken Imperiums - die Christianisierung von Staat und Gesellschaft - besitzen, sollen hier unter einigen grundlegenden Aspekten verfolgt und erhellt werden.

In der Tat lassen sich gewichtige Argumente gegen die Annahme eines historisch repräsentativen und aussagekräftigen Zeugniswert jener gut zwei Dutzend Texte vorbringen. ${ }^{4}$ Gerade dieser Abschnitt des Codex Theodosianus - wie überhaupt das gesamte 16. Buch, das sich mit Religions-, d.h. vor allem Kirchen- und Glaubensfragen befasst - ist aus der Perspektive der 420-430er Jahre zusammengestellt, also im Bewusstsein einer im Grunde bereits abgeschlossenen Christianisierung des Reiches verfasst. Der Kampf gegen das Heidentum - nicht hingegen der gegen häretische Gruppierungen - ist gewonnen, der retrospektive, ja historische Blick auf die Gesetze der Vorgänger wie überhaupt auf die unterlegenen traditionellen Kulte markant. ${ }^{5}$ Der inhaltliche Bogen der wiedergegebenen Gesetze - zunächst das Vorgehen gegen bestimmte Divinations- und Opferpraktiken, dann weitergehende Einschränkungen für Opferhandlungen, schließlich das Verbot des Tempelbesuchs in kultischer Absicht und endlich auch deren Profanierung oder Zerstörung - ist weitgehend geradlinig, gewissermaßen widerspruchsfrei. Gerade dieser Umstand verleiht den Aussagen dieser Texte zugleich auch einen stark normativen Charakter. Allerdings können jene wenigen Texte - es sind 25 aus dem Zeitraum 320 bis 435 n. Chr., also aus 115 Jahren - nur einen Ausschnitt aus jener undurchschaubaren Vielfalt von leges, edicta und Reskripten, die hier

3 So die traditionelle Herangehensweise an diese Rechtstexte: NoETHLichs 1971, DE GiovanNi 1980, GAUdEMET 1990, GAUdEMET 2000. Die Gesetze in Codex Theodosianus 16,10 sind nun in der vorzüglichen Ausgabe (mit frz. Übersetzung und detailliertem Kommentar) von DELMAIRE 2005 zu benutzen.

4 Dies zeigt bereits ein schneller Vergleich mit den beiden anderen mit dem Codex Theodosianus etwa zeitgleichen, unabhängig überlieferten zeitgenössischen Gesetzessammlungen, den Novellae Theodosii und besonders den Constitutiones Sirmondianae; letztere eine private Sammlung von 21 Konstitutionen des 4. und frühen 5. Jahrhunderts: VESSEY 1993; MATTHEWS 2000, 121167.

5 So heißt es bereits in CTh 16,10,22 (von $423 \mathrm{n}$. Chr.): paganos qui supersunt, quamquam iam nullos esse credamus, promulgatarum ....Iam dudum praescripta compescant. et cetera. Vgl. den folgenden Text, CTh 16,10,23 und dann den letzten des Kapitels (16,10,25, vom 14. Nov. 435), der, an dieser Stelle doppelt programmatisch, bestimmt: omnibus sceleratae mentis paganae exsecrandis hostiarum immolationibus damnandisque sacrificiis ceterisque antiquiorum sanctionum auctoritate prohibitis interdicimus cunctaque eorum fana templa delubra, si qua etiam nunc restant integra, praecepto magistratuum destrui collocationeque venerandae christianae religionis signi expiari praecipimus. 
hätten Berücksichtigung finden können, darstellen. ${ }^{6}$ Besaßen die Editoren des Codex auch die Anweisung, alle Gesetze von allgemeinem Charakter (generalitas) aufzunehmen, so haben praktische u.a. Hindernisse und Gründe dafür gesorgt, dass Gesetze unberücksichtigt blieben: Gerade im Bereich der Religionsgesetze lässt sich nachweisen, dass selbst durchaus einschlägige, sogar politisch eminent wichtige Texte keinen Eingang in den Codex fanden.

Doch ist es vor allem ein wenig beachteter Sachverhalt, der den Aussagewert dieser vielzitierten Religionsgesetze mit ihren detaillierten Bestimmungen als Zeugnisse einer koordinierten, universalen anti-paganen Politik in Frage stellt: Die überlieferten Texte stellen ausnahmslos nur gekürzte Fassungen der ursprünglichen kaiserlichen Verlautbarungen dar. Sie sind von den Editoren, wie alle im Codex aufgenommenen Texte, regelmäßig gerade derjenigen Bestandteile beraubt worden - der Skizze der Ausgangssituation, der Angabe des Initiativgrundes, auch der seitens des Kaisers mit ihrem Erlass verbundenen Motive -, die ihre Entstehung und konkrete Zielrichtung in ihrem historischen Kontext verorten und sie damit auch erst einer präzisen historischen Interpretation erschließen können. ${ }^{8}$

6 HONORÉ 1986, hier 161-168; MATTHEWs 2000, 63-68. 290. Die Herausgeber hatten Anweisung (CTh 1,1,5 pr.: cunctas colligi constitutiones decernimus, quas Constantinus inclitus et post eum divi principes nosque tulimus edictorum viribus aut sacra generalitate subnixas), in der Sammlung alle Gesetze mit dem Charakter von generalitas unabhängig von ihrer anhaltenden Gültigkeit aufzunehmen - was aber nachweislich nicht in jedem einzelnen Fall praktiziert wurde.

7 Der Ausschluss aller Religionsgesetze Julians aus dem Codex - sein berühmtes Lehrergesetz, in dem der Kaiser Christen verbot, die klassischen Texte zu unterrichten, ist allerdings enthalten (CTh 13,3,5; zu den Motiven siehe Julians eigene Erklärungen in ep. 61) - spiegelt dabei das christliche Anliegen des Gesetzeswerkes, doch fehlen etwa die historisch schlüsselhaften religionspolitischen leges generales Valentinians zu Beginn seiner Herrschaft, auf die der Kaiser selbst später, im Jahr 371, verweist (CTh 9,16,9): testes sunt leges a me in exordio imperii mei datae, quibus unicuique, quod animo inbibisset, colendi libera facultas tributa est (beachte LENSKI 2002, 215). Kein einziges der Gesetze, die das Ende der stadtrömischen Priesterschaften verfügten, ist in einer der Rechtsquellen überliefert, vielmehr nur in der literarischen Überlieferung bezeugt. Selbst das bedeutsame Dekret Gratians mit seinen Maßnahmen gegen Tempeln gehörende Liegenschaften (das im Streit um den Victoria-Altar vom Symmachus wie Ambrosius wiederholt angesprochen wird) ist nicht in einer Gesetzessammlung bewahrt. Auch bietet der Codex Theodosianus für den Zeitraum von 340 bis $380 \mathrm{n}$. Chr. kein einziges die jüdische Religionsgruppe betreffende Dokument, doch hatte Julian zwischenzeitlich (unbekannte) frühere anti-jüdische Maßnahmen aufgehoben. Die Liste dieser religionspolitischen Lücken ließe sich fortsetzen; es verlohnte, aus den verstreuten literarischen Erwähnungen und indirekten Indizien einmal eine umfassende (wenn auch niemals vollständige) Liste der in den großen Codices nicht berücksichtigten Gesetzestexte zur Religionspolitik zusammenzustellen und zu analysieren. BARNES 1984 führt auf der Basis von Eusebios einige konstantinische Religionsgesetze auf, die nicht überliefert sind - angesichts der Arbeitsweise Eusebs, Einzelmaßnahmen des ersten christlichen Kaisers eine allgemeine Bedeutung beizulegen, allerdings ein nicht unproblematisches Verfahren; vgl. ERRINGTON 1988; BRADBURY 1994, CURRAN 1996 (sowie CURRAN 2000, 169181).

8 Dies bedeutet das Grundsatzproblem jeglicher historischen Interpretation der im Codex enthaltenen Rechtstexte: Die entscheidenden spezifischen Informationen, welche das Verständnis einer Konstitution und ihrer Genese beförderten, sind radikal gekürzt, nur das Endprodukt - und dies in (in unbekanntem Umfange) kondensierter Form - bewahrt: und so teils überhaupt erst 
So hat Malcolm Errington in einer glänzenden Analyse das berühmte Edikt Theodosius' I. Cunctos populos vom 28. Februar 380 (CTh 16,1,2), welches nach allgemeiner Meinung die Erhebung des Christentums zur Staatsreligion verkünden soll, in seinen ursprünglichen historischen Zusammenhang plaziert und als regional begrenzte und präzisen kurzfristigen Zielen dienende Maßnahme des Theodosius im Zuge seiner Herrschaftsübernahme im Osten erweisen können.'

Ihren normativen Anstrich - also ihre programmatisch-allgemeine Zielrichtung - haben nicht wenige der im Codex bewahrten Texte zum Schicksal der Tempel unter den christlichen Kaisern erst aufgrund der Arbeit der Editoren angenommen: nämlich durch die Kürzung auf entscheidende Passagen nach dem Kriterium der generalitas, durch die getroffene Auswahl und die chronologische Reihung. Dies lässt sich bereits an den ersten beiden Texten im Kapitel De paganis, sacrificiis et templis zeigen. Markant programmatische Funktion - und deswegen an diese Stelle gerückt - hat der an zweiter Stelle aufgeführte Text, welcher die einprägsame, allgemeine' Erklärung cesset superstitio, sacrificiorum aboleatur insania bietet. Er ist aus einer ursprünglich selbstredend weit umfänglicheren, heute aber verlorenen bzw. wohl eher von den theodosianischen Juristen nicht weiter als erheblich befundenen Konstitution des Constantius II. des Jahre $341 \mathrm{n}$. Chr. herausgeschnitten - dies allerdings nicht ohne Schwierigkeiten. ${ }^{10}$ Jegliche Hinweise auf die näheren Umstände der Verlautbarung des Constantius fehlen. Vorangestellt ist ihr allein, wie so oft im Codex (der hier diesem Herrscher seine Reverenz als dem als maßgeblich betrachteten Gesetzgeber der Epoche erweist,) eine Konstitution Konstantins I. Diese allerdings - sie befasst sich mit der zulässigen Konsultation von Haruspices bei Naturereignissen (untersagt en passant aber immerhin häusliche Opfer) - ist ihrem Inhalt nach ungeachtet ihrer uns heute interessierenden historischen Aussage nicht von allzu großem Belang, zum Zeitpunkt der Zusammenstellung des Codex ohnehin bereits lange obsolet, aber eben konstantinisch. Der kurze Text des Constantius II. mit seinem

eine vermeintliche generalitas bewirkt. Eine ersten Schritt zur historischen Rekonstruktion erlauben die (seltenen) Fälle von Parallelüberlieferungen in Codex Theodosianus und Constitutiones Sirmondianae: letztere bewahren vollständige Texte. Siehe hierzu VESSEY 1993 und MATTHEWS 2000, 121-167, ,... the Sirmondian Constitutions claim the historian's attention because they preserve in their original form, or in something very close to it, constitutions that appear in edited form in the Theodosian Code“ (127). Ähnliche Einblicke erlauben auch die Novellae Theodosii der späteren Jahre; siehe hier beispielhaft die glänzende Analyse von Nov. Th. 3 durch MiLlar 2006, 120-123.

9 ERRINGTON 1997a. Vgl. hierzu jüngst MCLYNN 2005, hier 79-88.

10 CTh 16,10,2. Der nur dreißig Worte umfassende Eintrag bzw. Ausschnitt verweist vor allem auf ein älteres konstantinisches Gesetz, das allerdings nicht bewahrt wurde und offenbar von den Herausgebern des Codex nicht mehr aufgefunden werden konnte. Vollständig lautet der Gesetzeseintrag: IMP. CONSTANTIUS A. AD MADALIANUM AGENTEM VICEM P(RAEFECTORUM) $P($ RAETORI)O. cesset superstitio, sacrificiorum aboleatur insania. nam quicumque contra legem divi principis parentis nostri et hanc nostrae mansuetudinis iussionem ausus fuerit sacrificia celebrare, competens in eum vindicta et praesens sententia exeratur. ACCEPTA MARCELLINO ET PROBINO CONSS. Zur anti-paganen Politik Constantius II. siehe Leppin 1999. 
problematischen Verweis auf ein nicht mehr identifizierbares Gesetz aber suggeriert in seiner Kürzung und Prägnanz eben jenen gewünschten Charakter von generalitas, den er ursprünglich zweifelsfrei nicht besessen hat. ${ }^{11}$

Doch mit all dem nicht genug: Eine weitere Erkenntnis, ebenfalls von Malcolm Errington gewonnen, muss den modernen Historiker regelrecht verstören: In der zeitgenössischen Historiographie bleiben jene Gesetze so gut wie unbeachtet. Selbst die Kirchenhistoriker - obwohl immer bemüht, den Gang der Geschichte hin zum Sieg des wahren Glaubens und zum christlichen Imperium zu beschreiben und auf seine treibenden Kräfte hin zu befragen - widmen sich zwar ausführlich der Darstellung der Religionspolitik der Kaiser nach Konstantin, übergehen aber den Beitrag ihrer Gesetzgebung fast durchgängig mit Schweigen, messen ihr keine erkennbare Bedeutung im Kampf gegen Häresien oder Heidentum zu. Schlimmer noch: Sie scheinen die Gesetze oftmals überhaupt nicht zu kennen. ${ }^{12}$ Damit stellt sich unversehens die Frage nach der Bedeutung der anti-paganen Gesetzgebung in der Spätantike in ganz anderem Licht, also die Frage nach dem Stellenwert und der Wirksamkeit der normativen Verlautbarungen des Kaisers, bzw. die nach der Natur und Umsetzung der Religionspolitik in den Gemeinden des Imperiums und hier insbesondere ihre Rolle bei den seit den 380er Jahren einsetzenden gewaltsamen Übergriffen auf pagane Kultstätten in Städten des Ostens und Nordafrikas.

Zuvor aber noch einige Feststellungen zu den Bestimmungen im Codex Theodosianus 16,10: nämlich zur gesetzgeberischen Perspektive auf heidnische Tempel im Kontext der anti-paganen Religionsgesetzgebung. Im Mittelpunkt der Gesetze steht die Unterbindung öffentlicher paganer Praxis, insbesondere Divination und sacrificia. Tempel geraten als privilegierte Orte der Ausübung der heidnischen Kulte und hier vor allem der Durchführung blutiger Opfer (aber auch der Verehrung von Kultstatuen), in das Visier der Religionsgesetzgebung. Eine zweite, nicht minder wichtige Perspektive verfolgt demgegenüber ein völlig anders gelagertes Interesse: Sie betrachtet Tempel als repräsentative öffentliche Gebäude

11 Die gezwungene Einfügung dieses Textstückes erhellt auch ein bemerkenswertes Detail: Seine Adressierung ad Madalianum agentem vicem p(raefectorum) $p$ (raetori)o ist im Codex ohne Parallele. Regulär hätte der Text an einen Prätorianerpräfekten oder einen hohen Beamten auf der weiteren Hierarchie bis in die Provinz(en) gerichtet sein müssen. Lucius Crepereius Madalianus (PLRE I 540) war sehr wahrscheinlich vicarius Italiae (oder Africae). Vicarii figurieren sonst nicht als Adressaten anti-paganer Gesetze. Ein anderes Beispiel eines überzogenen, aber eben programmatischen Gesetzestextes, der an den Anfang eines Kapitels bzw. sogar eines Buches des Codex Theodosianus gerückt wurde, stellt CTh 16,1,2 dar.

12 ERRINGTON 1997b zur theodosianischen Religionsgesetzgebung. Dies gilt auch für Sozomenos, den Juristen unter ihnen, der nur eines von zahlreichen einschlägigen Religionsgesetzen des Theodosius I. nennt; ERRINGTON ebd. 21 möchte hier allerdings eine sorgfältige Auswahl erkennen. Dieser Befund mag allerdings teils auch der (in der Forschung oft unterschätzten) nur begrenzten geographischen Reichweite von Edikten geschuldet sein (welche die Exzerpte in den Codices nur ungenügend spiegeln). Vgl. allgemein zum Phänomen auch MATTHEWs 2000, 292:

"Contemporary - that is to say fourth- and early fifth-century - knowledge of the law was less comprehensive and well organized than the Code itself makes it appear." 
und erhebt sie aus ästhetischen Gründen, aber auch als traditionelle Bezugspunkte wichtiger öffentlicher Feste, zu Objekten gesetzlicher Schutzbestimmungen - so erstmals 342 in einem Gesetz des Constantius II. ${ }^{13}$ Die Unterbindung von Opfertätigkeit in Tempeln wird erstmals 346 (oder 354?) durch deren Schließung angeordnet; auf die Frage des Zugangs fokussieren dann die Texte bis zum Ende des 4. Jahrhunderts. ${ }^{14}$

Erst im Jahr 399, also viele Jahre nach den spektakulären Tempelzerstörungen unter der Regierung Theodosius I., spricht erstmals ein Gesetzestext von Zerstörung paganer Kultbauten: Die Niederreißung auf dem Lande gelegener paganer Heiligtümer soll erlaubt sein, sofern dies sine turba ac tumultu vonstatten gehen kann, um so die materielle Basis des Aberglaubens zu beseitigen. ${ }^{15}$ Der Bewahrung der öffentlichen Ordnung ist also unbedingte Priorität einzuräumen. Zeitlich folgende Bestimmungen zielen dann vor allem auf die Konfiskation paganer sakraler Stätten bzw. ihre Umwidmung zu profaner Nutzung, behandeln also vermögensrechtliche Fragen. Allein im Jahr 435 n. Chr., im letzten Text dieses Abschnitts des Codex ist dann erstmals davon die Rede, dass sämtliche noch unversehrten heidnischen Kultbauten zu zerstören bzw. durch Anbringung des Kreuzeszeichen zu desakralisieren seien. ${ }^{16}$ Demgegenüber wird die Schutzwürdigkeit gerade auch städtischer (zweifellos zentral gelegener) Tempel hervorgehoben und Tempel gar als ornamenta publicorum operum bezeichnet. ${ }^{17}$ Eine Politik der systematischen Zerstörung paganer Kultbauten ist im Codex Theodosianus mithin vor dem Jahre 435 nicht einmal ansatzweise formuliert: Die Zerstörung von Tempeln scheint überhaupt im Grunde außerhalb des kaiserlich-legislativen Blickfeldes zu liegen, das vielmehr städtebauliche und auch ökonomische Gesichtspunkte einbezieht. Es ist das Bemühen um Unterbindung der verbotenen Kulthandlungen, welcher die Tempel die repressive Aufmerksamkeit des Kaisers schulden.

Und es gibt darüber hinaus, viel zu wenig beachtet, eine weitere spezifische Zielrichtung, welcher die Tempel das gesetzgeberische Interesse des Kaisers jedenfalls unter der Perspektive der Editoren des Codex Theodosianus - verdanken,

13 CTh 16,10,3 (1. Nov. 342); vgl. auch CTh 16,10,17. Diese Gesetze stehen damit natürlich in der Tradition der Schutzbestimmungen für öffentliche (sowie länger bereits auch private, besonders Grab-) Bauten. Hierzu DALlY 2003, 98f. mit Lit. in Anm. 8. Der hier angelegte Zielkonflikt wird in zwei Gesetzestexten sogar explizit angesprochen, aber auch pragmatisch gelöst: Ungeachtet der Bekämpfung der paganen superstitio sollen Tempel unversehrt bleiben, doch ist auf die Unterdrückung jeglicher paganer Praktiken zu achten. CTh 16,10,15: Sicut sacrificia prohibemus, ita volumus publicorum operum ornamenta servari; CTh 16,10,17 (20. Aug. 399): Ut profanos ritus iam salubri lege submovimus, ita festos conventus civium et communem omnium laetitiam non patimur submoveri. Unde absque ullo sacrificio atque ulla superstitione damnabili exhiberi propulo voluptates secundum veterem consuetudinem, iniri etiam festa convivia, si quando exigunt publica vota, decernimus.

14 CTh 16,10,8 (382 n.), vgl. CTh 16,10,10 und 11 (391 n.); CTh 16,10,13 (395 n.).

15 CTh $16,10,16$.

16 CTh $16,10,25$.

17 CTh 16,10,15 (399 n.). Zu dieser Tempelschutzgesetzgebung GEYER, 1993; MEIER 1996. 
und auch nun gilt ihnen nur mittelbar das Interesse. Tatsächlich erreichen wir hier ein grundlegendes, ja strukturelles Problem der anti-paganen Gesetzgebung und Religionspolitik seit Konstantin. Mehreren Texten sind in ihrer Schärfe und Fokussierung bemerkenswerte Strafandrohungen angefügt: Sie offenbaren, dass der Kaiser die im Edikt inkriminierte Praxis nicht so sehr in der breiten Bevölkerung unterschiedslos verfolgen wollte (oder überhaupt diesen Gruppen primäre Bedeutung zumaß). Er hatte vielmehr einen spezifischen Personenkreis zuvorderst im Auge: den illustren Kreis der kaiserlichen iudices, also kaiserliche Spitzenfunktionäre (v.a. Statthalter und vergleichbare Amtsinhaber), die als Repräsentanten des Reiches die Verwaltung und Rechtsprechung in Provinzen und Städten wahrnahmen. ${ }^{18}$ Die Umsetzung kaiserlicher Erlasse und Politik oblag in entscheidender Weise diesem Personenkreis. Ohne dessen Mitwirkung, Initiative und entsprechend zielgerichtete Kooperation mit den städtischen Eliten im jeweiligen Amtsbereich blieb kaiserliche Politik wirkungslos. Erst recht die öffentliche Missachtung der offiziellen Religionspolitik durch den Vertreter des Kaisers - etwa persönlicher Tempelbesuch und Opfervollzug - in der Provinz musste hier verhängnisvolle Signalwirkung entfalten und den Beharrungswillen traditionalistischer Kreise beflügeln. ${ }^{19}$ Das Auftreten kaiserlicher Beamter sowie führender Persönlichkeiten in Stadt und Provinz war von entscheidender Bedeutung für den Machtanspruch und die Autorität des Kaisers in der Öffentlichkeit - und auch für die Glaubwürdigkeit seiner Religionspolitik im Reich.

Vergleichbare Strafdrohungen gegen Statthalter und ihr officium - als Kollektivhaftung und zur wirksamen gegenseitigen Kontrolle - sind auch sonst geläufig; doch allein im für Korruption besonders anfälligen Feld des Steuerwesens scheint sonst eine vergleichbar prononcierte kaiserliche Sanktionspolitik gegenüber der eigenen Verwaltung betrieben worden $\mathrm{zu}$ sein. ${ }^{20}$ Begünstigung und bewusste Untätigkeit von seiten der kaiserlichen Administration im Umfeld paganer und häretischer Religionspraxis werden in zahlreichen Quellen beklagt. Doch gehen hier die Vorwürfe über den der Bereicherungsabsicht hinaus und evozieren persönlichere Motive.

Fasst man die Aussage der in Codex Theodosianus 16,10 zusammengeführten Texte hinsichtlich ihrer Perspektive auf das Phänomen der Tempelzerstörung zusammen, so ist festzuhalten, dass zwar die Schließung von Tempeln als Mittel aktiver kaiserlicher Religionspolitik gegen pagane Kultaktivitäten betrieben wurde, nicht aber deren Zerstörung: Der Text vom Jahr 399 erörtert diese Möglichkeit, verdeutlich aber zugleich, dass hier heidnische Kreise christliche Übergriffe als illegale Handlungen angezeigt hatten. Die Konstitution räumt daraufhin halb-

18 ROSEN 1990, MEYER-ZWIFFELHOFFER in diesem Band.

19 MaCMuLLEN 1997, 24f.

20 Zusammenstellung bei NoETHLICHS 1981, 186ff. Unter den ca. 2.300 Gesetzen, die der Codex Theodosianus für den Zeitraum 312-437 n. Chr. enthält, umfassen etwa 100 auch Kontroll- und Bestrafungsklauseln gegenüber mit der Gesetzesdurchsetzung befassten Beamten. Tatsächlich dürften solche Zusätze aber weit häufiger gewesen sein; sie fielen der Redaktions- und Kürzungstätigkeit der Herausgeber des Codex aber leicht zum Opfer. 
herzig und mit explizitem Verweis auf den Vorrang der öffentlichen Ordnung die Zerstörung ländlicher Schreine ein, wobei eher an bereits faktisch aufgelassene Baulichkeiten gedacht zu sein scheint. Das Gesetz vom Jahr 435 wiederum zielt primär auf die Desakralisierung heidnischer Kultstätten - und erklärt seine Verwunderung darüber, dass es solche in unversehrtem Zustand überhaupt noch gebe. Auch hier ist eine offensive, ,destruktive' Intention anti-paganen kaiserlichen Vorgehens nicht recht erkennbar. Allein kaiserliche Politik, die Gefährdungen des öffentlichen Friedens in den Städten und Gemeinden des Reiches vermeidet, eignete sich - erst recht in der Rückschau des Jahres 438 - zur allgemeingültigen Norm.

Ist mit diesen Feststellungen nun alles zum Thema Gesetzgebung und Tempelzerstörung gesagt? Wohl nicht. So ist nur der Wortlaut der im Codex bewahrten Texte ist bislang befragt worden. Es fällt bereits auf, dass hier keine Rede ist von Bischöfen oder Mönchen, ${ }^{21}$ denen doch in unseren literarischen Berichten über Tempelzerstörungen entscheidende Rollen zugeschrieben werden - wo zudem aber auch kaiserliche Beamte agieren. Die Frage nach Eigenart und Zielen von Gesetzgebung kann nicht von der nach der Exekution von Recht, Gesetz und kaiserlicher Politik getrennt werden.

Welchen Geltungsanspruch erheben kaiserliche Gesetze oder Erlasse, welche normative, also allgemeingültige, räumlich wie zeitlich unbegrenzte Intention kaiserlichen Willens verbinden sich mit diesen Verlautbarungen? Grundlegende jüngere Untersuchungen (Honoré 1998, Harries 1999, Matthews 2000) haben deutlich gemacht, dass diese Frage die Voraussetzungen und den Charakter der spätantiken Gesetzgebung weitgehend verkennt. Gesetze sind nicht einseitige, politische Vorgaben des Kaiserhofes in Konstantinopel, sondern regelmäßig das Ergebnis eines komplexen Kommunikationsprozesses zwischen interessierten Parteien, Offizialen und Kaiserhof. ${ }^{22}$ Hierbei kommt suggestiones, die Präsentation von aktuellen Missständen oder Konflikten - inkl. Problemlösungsvorschlag eine außerordentliche Bedeutung zu. Fast alle kaiserlichen Konstitutionen im Codex Theodosianus wurden als Reskripte erlassen, angestoßen von suggestiones aus dem Instanzenzug der kaiserlichen Verwaltung, aber auch von Interessengruppen oder einflussreichen Persönlichkeiten und Gesandtschaften in der Provinz. Die vorgetragenen Fälle mochten weiterführende Probleme berühren und ,politische' Bedeutung besitzen, waren aber doch regelmäßig aus spezifischen Problemkonstellationen erwachsen, deren Lösung das schließlich, am Ende eines langen Konsultationsprozesses, erlassene Reskript mit seiner Entscheidung dann bewirken musste. In ein Edikt, auch in eine lex generalis, flossen ebenso Vorgaben der Gesetzestradition, Entscheidungen von Vorgängern, Intentionen aktueller Politik, verfügbare Informationen, Konfliktkonstellationen, vor allem aber die Interessen und Einflussnahmen der an seiner Entstehung beteiligten Gruppen,

21 FREND 1990; FOWDEN 1978; HAHN 2004, 102f. 110. 278; GADDIS 2005.

22 HARRIES 1999, 36: "Although dirigiste in its language, imperial general law was in fact more often negotiated than imposed." 
Beamte und Offiziale ein. Gesetze als Resultat eines intensiven Kommunikationsund Aushandlungsprozesses zwischen Initiatoren, Betroffenen, verschiedenen Instanzen der kaiserlichen Verwaltung bis hin zum consistorium und dem Kaiser selbst stehen somit zugleich für kommunizierte Politik. Und sie inszenieren Herrschaft, selbst wenn die publizierten kaiserlichen Konstitutionen nur Bestimmungen wiederholen oder oft nichts anderes als die de facto Anerkennung von bereits vor Ort etablierten Sachverhalten oder Verfahrensweisen ex post bedeuteten. Gesetze dienten so einerseits der Regulierung lokaler oder regionaler Fragen und mussten ihrem Empfänger, dem Statthalter oder iudex, eine hinreichend klare und konkrete Handreichung zur Entscheidung der ihm vorgelegten Problemfälle bieten; andererseits artikulierten sie auch Zielvorstellungen und Richtlinien für das weitere Agieren der kaiserlichen Verwaltung, formulierten insofern Politik und entwickelten einen Bezugsrahmen für den Umgang mit zukünftigen Entscheidungsfällen.

Die in zahlreichen Konstitutionen aufscheinende bemerkenswerte Gelassenheit des kaiserlichen Gesetzgebers gegenüber der unzureichenden Umsetzung seiner Maßgaben, der Untätigkeit, Versäumnisse, Irrtümer und Verfehlungen der iudices und officiales unterstreicht auch die in der Praxis verbleibenden - teils nicht kontrollierbaren - exekutiven Spielräume in Provinzen und Städten. Die konkrete Situation, die hier wirksamen Zwänge und Kräftekonstellationen, insbesondere auch die Durchsetzungsfähigkeit des jeweiligen Amtsträgers vor Ort, entschieden mit über die Realisierungsmöglichkeiten kaiserlicher Politik.

Die Eigenart kaiserlicher Gesetzgebung als kontinuierlicher Kommunikations- und Aushandlungsprozess erklärt zudem ihre erstaunliche Flexibilität im Einzelfall. Mochten die Kaiser Valens und Valentinian im Jahre 364 auch nächtliche Opfer verboten haben - das Edikt ist im Codex Theodosianus unter 9,15,7 bewahrt -, so hinderte dies doch den Prokonsul von Achaea, den stadtrömischen Senator und bekannten Heiden Vettius Agorius Praetextatus nicht daran, dem Kaiser vorzutragen, dass dieses Gesetz den Bewohnern Griechenlands das Leben unerträglich mache: auf göttlichen Anweisungen beruhten nämlich ihre nächtlichen Riten bei den heiligen Mysterien, und diese Bräuche stellten doch den Fortbestand des Menschengeschlechts insgesamt sicher! Valentinian verzichtete angesichts solcher Argumentation auf die Durchsetzung des Edikts und gestattete die Fortführung der altväterlichen Riten. ${ }^{23}$

Das vor Ort - in Stadt, Provinz oder Region - herrschende Kräfteverhältnis, die Konstellation der hier agierenden kaiserlichen Beamten, Magistrate und Interessen rückt somit in den Fokus. Viel spricht dafür, dass iudices hier aus mancherlei Gründen selten die aktivste Rolle spielten, also die Initiative ergriffen, wenn Missstände zu beseitigen oder Konflikte zu lösen waren. Allerdings waren sie Ansprechpartner interessierter Personen und Kreise. Und ebenso wie die iner-

23 Zosim. 4,3,2f. Zur Stelle und zum historischen Zusammenhang siehe F. PASCHOUD (ed.), Zosime, Histoire Nouvelle Livre IV, n. 111 336ff. ad locum. Beachte auch RougÉ 1967 sowie G. FOWDEN in JRA 8 (1995), 549. 
tia, die Untätigkeit von Statthaltern in nahezu allen Tätigkeitsfeldern jenseits der Steuerverwaltung ihrer Provinz regelmäßig in den Quellen beklagt wird, so ist es seit konstantinischer Zeit offensichtlich, welche neue Institution in der Stadt nun gesellschaftliche, und zwar für die gesamte Bürgerschaft - nicht eine einzelne Interessengruppe - Verantwortung übernimmt: Dem örtlichen Bischof wird, neben der audientia episcopolis, durch den Kaiser vor allem auch ein außerordentliches Suggestionsrecht explizit eingeräumt - also privilegierter Zugang zum System der Gesetzgebung. ${ }^{24}$ Augustinus beklagt in seinen Briefen, welche Verantwortungsbereiche der Statthalter und die städtischen Magistrate im Alltag regelmäßig nicht ausfüllen oder wahrzunehmen überfordert sind: Fälle von Vergewaltigung, auch illegaler Sklavenhandel und sogar Kindesraub - all dies bleibt ohne staatliche Verfolgung und Sanktion, so dass er, der Bischof, sich dieser Rechtsfälle anzunehmen hat, aber auch etwa die Bestellung eines defensor civitatis initiieren muss. ${ }^{25}$ Solche Verhältnisse spiegeln den schlichten Sachverhalt ,that imperial government was, except in a few cities serving as seats of governors ... more absent than present. ${ }^{\prime 26}$

Augustinus eignete sich in seinem Amt nicht nur eine beachtliche Rechtskenntnis an: Zur Ausfüllung seiner episkopalen Pflichten legte er sich auch ein Archiv mit kaiserlichen Konstitutionen an, versorgte in Rechtsstreitigkeiten verwickelte Kollegen mit Kopien und warnte ggf. auch untätige iudices unter diskreter Beilage der einschlägigen Gesetzestexte. ${ }^{27}$ Am Kaiserhof in Ravenna schließlich ergriff er mit Unterstützung seines Freundes Alypius die Initiative zur Ausfertigung eines wirkungsvollen Gesetzes zum Schutz geraubter ingenui vor Versklavung. ${ }^{28}$ Einem energischen Bischof fiel schnell die Bekämpfung zivilrechtlicher Missstände zu - und er verfügte notfalls auch über Erzwingungsmittel, diese durchzusetzen: Bischof Antoninus in Fussala benutzte seinen defensor ecclesiae, dazu städtische vigiles, am Ort stationierte Soldaten und sogar Angehörige des Klerus; der Patriarch von Alexandria, Kyrill, verfügte mit den 600 parabalani (Krankenträgern) sogar über einen handfeste Truppe, die selbst den Stadtrat der ägyptischen Metropole einschüchterte. ${ }^{29}$

24 HARRIES 1993, 12; HUNT 1993, 151-154.

25 LENSKI 2001, 92 und DOSSEY 2001 mit den Belegen.

26 BagNAll 1993, 62 zur Situation in ägyptischen Städten. Ebd. 64ff. ausgezeichnete Beobachtungen zur Präsenz und Tätigkeit des Statthalters, u.a. mit Verweis auf dessen minimale Personalausstattung. Zur bescheidenen Macht spätantiker Statthalter BROWN 1992, 22ff. - zu derjenigen in der oberägyptischen Thebais angesichts eigenmächtiger Aktionen des Abtes Schenute BEHLMER 1993; HAHN 2004, 255-260.

27 HARRIES 1999, 92; LeNSKI 2001, 88f. HuMFRESS 2007, 190f. Zur Problematik lokaler bzw. statthalterlicher Archive und der Verfügbarkeit von Gesetzen MATTHEws 2000, 281-286 sowie DE STE. CROIX 2006, 222.

28 BROWN 22000, 470.

29 LENSKI 2001, 92; DOSSEY 111: Kleriker des Augustinus peitschten gar einen Kurialen aus, den sie beim Übergriff auf eine Nonne ergriffen. Zum weiteren aufschlußreichen Streit um diesen Fall - und die Haltung des Augustinus dazu - DOSSEY 2001, 111f. Alexandria: HAAS 1997, 235-238; HAHN 2004, 113-120; beachte nun BOWERSOCK 2010. 
Vor allem der privilegierte Zugang des Bischofs - erst recht einer Persönlichkeit mit präzisen politischen Informationen und genauer Kenntnis der aktuellen Rechtslage - zu Instanzen der Reichsverwaltung, vom Statthalter und seinem officium bis hin zum Kaiserhof und dem Herrscher selbst, eröffnete außerordentliche Möglichkeiten der Einflussnahme auf Informationsfluss, Verwaltungsvorgänge, Entscheidungsvorlagen und auf die schließliche Realisierung kaiserlicher Entscheidungen - und all dies im Einzelfall auch unter Umgehung ggf. widerstrebender Zwischeninstanzen. Im besonderen konnte ein Bischof, ggf. mit Hilfe der Netzwerke der Kirchenorganisation in seiner Provinz und am Hof, auch die Umsetzung existierender gesetzlicher Richtlinien gegen lokale Widerstände und selbst dort etablierte Machtverhältnisse betreiben, notfalls auch ohne Einverständnis der Repräsentanten der kaiserlichen Verwaltung. Denn im politischen Alltag auf lokaler Ebene entschied regelmäßig der soziale Konsens unter den etablierten Eliten und dominierenden potentes über das Agieren der Magistrate, d.h. aber auch über die konsequente Umsetzung herrschenden Rechtes - oder dessen Verwässerung, Verzögerung, ja Missachtung.

Interventionsmöglichkeiten - bis hin zur Zerstörung von noch frequentierten Tempeln - eröffneten sich einem machtbewussten Bischof, wenn lokale Konstellationen von starken sozialen und religiösen Gegensätzen, latenten Spannungen oder gar offenen Konflikten und Gewalt gekennzeichnet waren. ${ }^{30}$ Die Störung des öffentlichen Friedens, dessen Bewahrung noch vor der Sicherstellung einer reibungslosen Steuererhebung Primat römischer Herrschaft war, bot Kirchenkreisen den besten Ansatzpunkt, um zugleich religiöse Missstände - darunter auch die Benachteiligung der eigenen Gemeinde - anzuprangern und ihre Abstellung mit aller Konsequenz, d.h. unter Beseitigung auch der strukturellen Ursachen, zu verlangen. Ging solche Störung zugleich mit offener Missachtung kaiserlicher Gesetzgebung einher, bedeutete es für Instanzen der kaiserlichen Verwaltung ein erhebliches Risiko, Initiativen zu verzögern bzw. Sympathie oder offenes Einvernehmen mit den Interessen der lokalen Eliten durch Untätigkeit oder Verschleppung zu demonstrieren. Alle gewaltsamen Tempelzerstörungen der theodosianischen Zeit lassen sich auf solche Konstellationen zurückführen. Verschiedentlich ist auch eine von Kirchenkreisen bewusst betriebene Eskalation der örtlichen Situation zu erkennen.

Hagiographische Quellen - insbesondere die Vita Porphyrii mit ihrer Schilderung der bischöflichen Agitation am Kaiserhof zur Erwirkung eines Edikts zur Zerstörung der Tempel in Gaza ${ }^{31}$ - bieten Szenarien, wie gegen den Widerstand

30 HAHN 2004, 276ff.; HAHN 2008.

31 Die zweifache Gesandtschaftsreise des Bischofs von Gaza, Porphyrios, und seines Metropolitan von Jerusalem nach Konstantinopel in den Jahren um $400 \mathrm{n}$. Chr., die dortige Agitation am Hof und später die Umsetzung der erwirkten Beschlüsse und Machtmittel (zur Zerstörung der Tempel Gazas) repräsentieren das zentrale Geschehen dieser hagiographischen Überlieferung. Zu ihrem historischen Wert siehe TROMBLEY I 1993, 246-282; HAHN 2004, 202-207; CHILDERS 2001. Das letzte Wort zur Authenizität und Aussagekraft dieser wichtigen Quelle ist noch nicht gesprochen. 
von Bevölkerung und städtischer Aristokratie (und ohne Einbeziehung der provinzialen und regionalen Instanzen der römischen Herrschaft) Machtmittel von außen gewonnen und eingesetzt werden konnten. Die hier geschilderten Vorgehensweisen lassen sich aber auch in besser bezeugten historischen Konstellationen en detail nachweisen: Die schließliche Intervention des Kaisers im kleinen nordafrikanischen Calama beruhte auf eben dieser skizzierten Verbindung von lokaler stasis, Unterdrückung der christlichen Minorität und der offenen Missachtung kaiserlichen Willens. Das den Vorfall und die damit verknüpften administrativen Vorgänge aufgreifende kaiserliche Edikt, welches - eine glückliche Fügung der Überlieferung - sogar vollständig auf uns gekommen ist, kritisiert scharf die Untätigkeit der iudices in ganz Nordafrika. ${ }^{32}$

Bischöfliche Initiativen zur Schließung oder gar Zerstörung von Tempeln operierten immer im Kontext der allgemeinen religionspolitischen Direktiven der christlichen Kaiser. Zugleich wussten sie sich aber auch deren rhetorische Zuspitzungen zunutze zu machen. Wenn es zutrifft, dass viele Gesetze, gerade Religionsgesetze - wie es Sozomenos für diejenigen des Theodosius I. (wie zuvor schon für Konstantin) ausdrücklich behauptet ${ }^{33}$ - primär abschreckend wirken sollten, aber in ihrer aggressiven Formulierung noch keine konkrete Anwendung vor Augen hatten, dann ist die in einzelnen Fällen und unter spezifischen lokalen Voraussetzungen von Bischöfen am Kaiserhof eingeforderte Exekution das entscheidende Moment im Prozess der episkopalen Bekämpfung des Heidentums. Ziel der kirchlichen Agitation am Kaiserhof war regelmäßig die Gewinnung einer auf die konkrete lokale Situation bezogenen Ausfertigung, zugleich pragmatischen und vor allem handlungsbezogenen Fokussierung der bekannten allgemeinen religionspolitischen Direktiven der regierenden Herrscher. Und so bedeutet die Äußerung des Theodoret über Bischof Marcellus von Apamea, dieser habe ,als erster das Gesetz als Waffe verwendet“ ( $\pi \lambda \quad \tau \quad$ vó $\mu \quad \chi \rho \eta \sigma \quad \mu \varepsilon v o \varsigma),{ }^{34}$ als er den Tempel der Stadt von kaiserlichen Beamten und Militär habe zerstören lassen, nichts anderes, als dass er einen kaiserlichen Erlass hatte erwirken bzw. eine entsprechende Sondermission nach Apamea hatte leiten können.

Es ist ungemein aufschlussreich, dass die Umsetzung solcher spezifischen kaiserlichen Dekrete im Falle von Tempelzerstörungen (oft auch bei Tempelschließungen) offenbar nie auf dem regulären Instanzenzug erfolgte: Wir hören keinen Statthalter solch einschneidende Direktiven in spektakulärer Aktion umsetzen. Es sind, sieht man von dem einmaligen Fall des Prätorianerpräfekten Cynegius ab (allerdings mit eigens formuliertem Auftrag), ${ }^{35}$ in aller Regel Sonderbe-

32 Const. Sirmond. 12 und 14. Zu den Vorgängen Harries 1999, 88-92; HERMANOWICZ 2004 (jetzt als ch.4 in HERMANOWICZ 2008) und MEYER-ZWIFFELHOFFER 93-96 im vorliegenden Band.

33 Sozom., H.E. 2,32.

34 Theodoret., H.E. 5,21,5. Zur Übersetzung (und Deutung) vgl. aber ERRINGTON 1997, 408, n.50.

35 CTh 16,10,9. Auch wurde ein erheblicher Teil der Religionsgesetze des Theodosius Cynegius ausgestellt. Siehe MARIQUE 1963, 52 sowie die Zusammenstellung in PLRE I, 1971, 235f., s.v. Cynegius 3. 
amte, notarii u.a. eigens mit diesem Projekt beauftragte Amtsträger, die solche tief in den inneren Frieden von Gemeinden hineinreichende Maßnahmen wie eine vor Ort mehrheitlich vehement abgelehnte Schließung oder Zerstörung eines wichtigen Heiligtums umzusetzen hatten. Nicht auf dem hierfür bestimmten regulären Instanzenzug, vom consistorium über den Prätorianerpräfekten zu den Stadtpräfekten und Statthaltern, erfolgte die Umsetzung von offensiver und strittiger kaiserlicher Religionspolitik, sondern per Spezialmission unter expliziter Autorisierung durch den Kaiser und dies sogar unter Einsatz von eigens hierfür detachierten militärischen Einheiten. ${ }^{36}$

Bischöfe nutzten mithin die mit der Existenz von allgemeinen Religionsgesetzen und anti-paganer Gesetzgebung vorgegebenen Spielräume im Kampf gegen noch blühende heidnische Kulte (weit mehr aber noch gegen konkurrierende christliche Gruppierungen!). Sie erwirkten spezifische Erzwingungsmaßnahmen gegen einzelne Kulte: dies aber nicht ohne Widerstand am Kaiserhof und sogar der Person des Herrschers selbst, der um die politischen Risiken und vor allem drohenden fiskalischen Kollateralschäden solcher Aktionen wohl wusste. ${ }^{37}$ Solches Vorgehen sollte dabei nicht als eine spezifisch im Kampf gegen pagane Kulte und Tempel entwickelte Strategie betrachtet und hierdurch überbewertet werden. In der nordafrikanischen Kirche war die Einschaltung (respektive Instrumentalisierung) der Staatsmacht eine schon lange geübte, ja bewährte Praxis: In den schweren, jahrzehntelangen Auseinandersetzungen mit den Donatisten - auch diese von katholischer Seite unaufhörlich als Gefährdung der öffentlichen Ordnung stigmatisiert - wurde immer wieder an Konstantinopel appelliert und die Entsendung von Sonderbeamten samt Militär gefordert und erwirkt, letztlich diese konkurrierende Kirchenorganisation mit staatlichen Zwangsmitteln unterdrückt. ${ }^{38}$ Anfang des 5. Jahrhunderts verlegten sich dann nordafrikanische Synoden darauf, in sukzessiven Konzilsbeschlüssen vom Kaiser die Zerstörung bestimmter Tempel zu fordern. Das Bemühen um Legalität, um explizite Autorisierung von Gewaltanwendung ist dabei markant. ${ }^{39}$

36 Dieses Vorgehen, der Einsatz von Sonderbeamten, lässt sich im übrigen auch im Umgang mit öffentliche Belange berührenden innerkirchlichen Konflikten, insbesondere in den großen Metropolen des Ostens (auch aber auch im donatistischen Schisma) feststellen: ISELE 2010. Für die Zerstörung des Heidentums in Gaza und das hierbei eingesetzte Personal siehe V. Porph. 27 (mit der Literatur in Anm. 31).

37 Arcadius soll das Verlangen der nach Konstantinopel gereisten Bischöfe, die Zerstörung aller paganen Kultstätten Gazas zu befehlen und die christliche Gemeinde statusmäßig zu privilegieren, zunächst mit der Begründung abgelehnt haben: „Ich weiß wohl, dass diese Stadt dem Götzendienst ergeben ist; doch erfüllt sie loyal ihre Steuerpflichten und erbringt hohe Einkünfte.“ (V. Porph. 41). Von Schenute in Panopolis bedrängte Heiden forderten ihn auf, sie vor dem Gericht des Statthalters zu verklagen und erklärten „Wir bezahlen Steuern!“; BEHLMER 1996, XC. BROWN 1963; FREND 1989; GAUDEMET 2000; HERMANOWICZ 2008, $97 \mathrm{ff}$.

39 Das Material hierzu, das die schrittweise Ausweitung der Forderungen der afrikanischen Kirche bezüglich anti-paganer Maßnahmen an den Kaiserhof ausweist, findet sich in den Konzilsakten der karthagischen Synoden; siehe nur Conc. Carth., 13 Iun. 407 = Reg. Eccl. Carth. Excerpt. XII 97 (CCL 149: 215, Munier); Conc. Carth., 16 Iun. 408 = Reg. Eccl. Carth. Excerpt. XIII (CCL 
Die kaiserliche Gesetzgebung bot im Kontext der offensiven kirchlichen Auseinandersetzung mit paganen Kulten (oder Häretikern) einen ambivalenten juristischen Bezugsrahmen. Als programmatische Verlautbarung kaiserlicher Religionspolitik bedeutete sie in der Realität der Reichsverwaltung eben nicht die zwingende Richtschnur konsequenten administrativen Handelns in allen Regionen und Städten des Imperiums. Allerdings bot sie beachtliche Spielräume zum Tätigwerden vor Ort, entsprechende Konstellationen, Initiativen und Einflussmöglichkeiten vorausgesetzt. Gerade deshalb war es im Einzelfall wohl sogar möglich, auf die aufwendige und zeitraubende Einholung eines konkreten kaiserlichen Dekrets zu verzichten: solange die vor Ort gewählte Vorgehensweise im Rahmen der existierenden gesetzlichen Normen nur plausibel war. Die Zerstörung des Sarapeion von Alexandria im Jahr 392 n. Chr., die von Christen wie Heiden der Zeit unmittelbar als Fanal und Epochenschnitt interpretiert wurde, erfolgte offenbar nicht auf der Basis eines kaiserlichen Ediktes, auch wenn dies in der kirchenhistorischen Überlieferung, v.a. vom Zeitgenossen Rufinus behauptet wird. ${ }^{40}$ Doch bereits der von ihm paraphrasierte Text ist abstrus, fügt sich zudem auch nicht in den angeblichen Verlauf der Eskalation der Gewalt ein und wirft weitere Probleme auf. Den Legitimationsgrund für den Angriff auf das Sarapis-Heiligtum (samt dessen späterer Zerstörung) lieferte die in Alexandria entstandene Situation offener stasis, in der der Sarapis-Tempel als Bastion der paganen Kämpfer gedient haben soll. Dass diese religiös motivierte Bürgerkriegssituation - wenn es denn eine solche überhaupt gab - durch Provokationen des Bischofs Theophilos ausgelöst worden war, spielte später, nach der Eskalation des Geschehens und den ersten Toten, keine Rolle mehr. Der Jurist und Kirchenhistoriker Sozomenos, der dieses angeblich inmitten der Unruhen verlesene, aus Konstantinopel eingeholte kaiserliche Zerstörungsdekret des Theodosius in den Archiven des Hofes suchte, um daraus zitieren zu können, konnte es jedenfalls nicht ausfindig machen. Auch die Beteiligung der führenden kaiserlichen Beamten in Alexandria an den Zerstörungen erfolgte wohl ohne Wissen und Autorisierung des Theodosius. Für die Eruption der Gewalt in Alexandria und das Eingreifen der Repräsentanten der kaiserlichen Macht blieb dies aber ohne Bedeutung: Der Kaiser war im Handeln seiner Beamten und Soldaten allen Beteiligten der Auseinandersetzungen gegenwärtig.

Zeitgenossen ,erfuhren' die Gesetze - und die Machtvollkommenheit! - ihres Herrschers zweifellos gerade in solchen Konstellationen und Momenten, wenn leges sacrae oder Edikte, anstatt geräuschlos nach einem der Allgemeinheit nicht einsichtigen oder nachvollziehbaren langwierigen Entstehungs- und Entscheidungsprozess schließlich vor Ort in einem konsensualen Verfahren unter Beteiligung der lokalen Elite und einflussreicher Persönlichkeiten geräuschlos umgesetzt

149: 219, Munier); Conc. Carth., 13 Oct. 408 = Reg. Eccl. Carth. Excerpt. XIV (CCL 149: 219, Munier).

$40 \mathrm{Zu}$ den Quellen - es existiert eine erstaunlich dichte und vielfältige Überlieferung - und diesen Vorgängen wie auch der Chronologie siehe eingehend Hahn 2006 und 2008. 
(oder auch ignoriert) zu werden, vielmehr in einer spektakulären Inszenierung von Macht evoziert wurden: unter Beteiligung kaiserlicher Instanzen oder doch von Handelnden, die sich auf den kaiserlichen Willen beriefen. Zur offensiven Durchsetzung religionspolitischer Ziele und Partikularinteressen auch gegen lokale Widerstände bedurfte es unter den Bedingungen dieser Gesellschaften aber mehr als des Rekurses auf die geltende Rechtslage. Initiative und weitreichende Beziehungen waren notwendig, um den staatlichen Machtapparat tatsächlich zum Handeln zu veranlassen - vor allem bedurfte es regelmäßig der Autorisierung durch den Kaiser selbst, und dies nicht nur deshalb, weil ohne seine Exekutionsmittel die gewaltsame Unterdrückung des Heidentums kaum möglich war.

Darüber hinaus gilt: Kaiserliche Gesetze und Verlautbarungen waren in der Spätantike in einem unmittelbaren Sinne omnipräsent: an den Schauwänden der öffentlichen Plätze, in den Gerichtssitzungen der Statthalter. Präsent und tief ins Bewusstsein der Bevölkerung eingebrannt waren sie aber vor allem auch - und dies ist dem modernen Betrachter weit schwerer nachzuvollziehen - durch die Art ihrer Verkündigung: Die ,Publikation' eines neuen Gesetzes, dessen Eintreffen in der Stadt sich bereits wie ein Lauffeuer herumgesprochen haben mochte, erfolgte durch den Statthalter oder den höchsten städtischen Beamte als außerordentliches, dramatisch inszeniertes Ereignis in der Öffentlichkeit: Seine laute Verlesung vor der ängstlich schweigenden Menge - so lassen es uns Schilderungen wissen ${ }^{41}$ vollzog sich in einer beklemmenden Atmosphäre: einer der seltenen und einschüchternden Momente, in denen Bürger ihren Herrscher, erlebten' und seinem Willen lauschen durften. Diese Schilderungen reflektieren dabei exakt jene Empfindungen, die Schenute in seiner eingangs zitierten Predigt bei seinen Hörern im oberägyptischen Panopolis ansprach. Und es sind dieselben äußeren Umstände, die Honorius wachruft, wenn er mit den ,tausend Schrecken der Gesetze' (legum mille terrores) droht, die seiner Religionspolitik, welche der Vernichtung von omnis superstitionis materia - gemeint ist das Heidentum und hier erstmals seine bauliche Substanz, die Tempel - die nötige Durchsetzungskraft verleihen werden. ${ }^{42}$ Für den Historiker ist es besonders schmerzlich, dass die kaiserliche Rhetorik, die im speziellen solchen Schrecken verbreitete und in den einleitenden Abschnitten der Gesetze breiten Raum einnahm, in nahezu allen Fällen den Scheren der Herausgeber des Codex Theodosianus zum Opfer gefallen ist. ${ }^{43}$

41 Siehe HARRIES 1999, 70ff.; MATTHews 2000, 187ff. mit den Belegen.

42 CTh 16,10,16 bzw. Nov. Th. 3. Der Begriff superstio gilt hier ausschließlich paganer Kultausübung - oder klassifiziert solche als superstitio. Vgl. HUNT 1993, 145 und SALZMAN 1987.

43 Siehe hingegen den vollständig erhaltenen Text von Nov. Th. 3 mit MiLlar 2006, 120-123. 


\section{Bibliographie}

Bagnall, R.S., Egypt in Late Antiquity, Princeton 1993.

Barnes, T.D., Constantine's Prohibition of Pagan Sacrifice, AJPh 105 (1984), 6972.

Barnes, T.D., Christians and Pagans in the Reign of Constantius, in: Vittinghoff, F. - Dihle, A. (Hrsg.), L'Eglise et l'empire au IV siècle (Entretiens Hardt 34), Vandoeuvres - Genève 1989, 301-343.

Behlmer, H., Historical Evidence from Shenoute's De extremo iudicio, in: Sesto Congresso internazionale di Egittologia, Atti II, Torino 1993, 11-19.

Behlmer, H. (Hrsg.), Schenute von Atripe: De iudicio (Torino, Museo Egizio, Cat. 63000, Cod. IV). Catalogo del Museo Egizio di Torino, Serie Prima Monumenti e Testi, vol. VIII, Torino 1996.

Bowersock, G.W., Parabalani: a Terrorist Charity in Late Antiquity, Anabases. Traditions et réception de l'Antiquité 12 (2010), 45-54.

Bradbury, S., Constantine and the Problem of Anti-Pagan Legislation in the Fourth Century, CPh 89 (1994), 120-139.

Brown, P., Religious Coercion in the Later Roman Empire: the Case of North Africa, History 48 (1963), 283-305.

Brown, P., Power and Persuasion in Late Antiquity. Towards a Christian Empire, Madison 1992.

Brown, P., Christianization and Religious Conflict, in: Cameron, A. - Garnsey, P. (Hrsg.), The Cambridge Ancient History. Bd. XIII: The Late Empire, A.D. 337-425, Cambridge 21998, 632-664.

Brown, P., Augustine of Hippo, Berkeley - Los Angeles ${ }^{2} 2000$.

Childers, J.W., The Georgian Life of Porphyry of Gaza, StudPatr 35 (2001), 374384.

Curran, J.R., Constantine and the Ancient Cults of Rome. The Legal Evidence, Greece \& Rome 43 (1996), 68-80.

Curran, J.R., Pagan City and Christian Capital. Rome in the Fourth Century, Oxford 1999.

De Giovanni, L., Chiesa e Stato nel codice Teodosiano. Saggio sul libro XVI, Napoli 1980.

Delmaire, R. (Hrsg.), Les lois religieuses des empereurs romains de Constantin à Théodose II (312-438), vol. I: Code Théodosien Livre XVI (Sources Chrétiennes 497), Paris 2005.

Dossey, L., Judicial Violence and the Ecclesiastical Courts in Late Antique North Africa, in: Mathisen, R.W. (Hrsg.), Law, Society, and Authority in Late Antiquity, Oxford 2001, 98-114.

Emmel, S., From the Other Side of the Nile: Shenute and Panopolis, in: Egberts, A. - Muhs, B.P. - Vliet, J. van der (Hrsg.), Perspectives on Panopolis. An Egyptian Town from Alexander the Great to the Arab Conquest, Leiden Boston - Köln 2002, 95-113. 
Emmel, S., Shenoute of Atripe and the Christian Destruction of Temples in Egypt: Rhetoric and Reality, in: Hahn, J. - Emmel, St. - Gotter, U. (Hrsg.), From Temple to Church: Destruction and Renewal of Local Cultic Topography in Late Antiquity (Religions in the Graeco-Roman World 163), Leiden 2008, 161-201.

Ensslin, W., Die Religionspolitik des Kaisers Theodosius des Großen, München 1953.

Errington, R M., Constantine and the Pagans, GRBS 29 (1988), 309-318.

Errington, R.M., Church and State in the First Years of Theodosius I, Chiron 27 (1997), 21-72. [= 1997a]

Errington, R.M., Christian Accounts of the Legislation of Theodosius I, Klio 79 (1997), 398-443. [= 1997b]

Errington, R M., Roman Imperial Policy from Julian to Theodosius, Chapel Hill 2006.

Fowden, G., Bishops and Temples in the Eastern Roman Empire A. D. 320-435, JThS 29 (1978), 53-78.

Frend, W.H.C., Augustine and State Authority. The Example of the Donatists, in: Agostina d'Ippona "Quaestiones Disputatae", Palermo 1989, 49-73 = ders., Orthodoxy, Paganism and Dissent in the Early Christian Centuries (CSS 750), Aldershot 2002, XII.

Frend, W.H.C., Monks and the End of Greco-Roman Paganism in Syria and Egypt, CrSt 11 (1990), 469-484.

Gaudemet, J., La législation anti-païenne de Constantin à Justinien, CrSt 11 (1990), 449-468.

Gaudemet, J., La politique religieuse impériale au $\mathrm{IV}^{\mathrm{e}}$ siècle (envers les païens, les Juifs, les hérétiques, les donatistes), in: Gaudemet, J. - Siniscalco, P. Falchi, G.L. (Hrsg.), Legislazione imperiale e religione nel IV secolo, Roma 2000, 7-66.

Gaudemet, J. - Siniscalco, P. - Falchi, G.L. (Hrsg.), Legislazione imperiale e religione nel IV secolo (Sussidi Patristici 11), Roma 2000.

Geyer, A., „Ne ruinis urbs deformetur ...“. Ästhetische Kriterien in der spätantiken Baugesetzgebung, Boreas 16 (1993), 63-77.

Haas, C., Alexandria in the Late Antiquity: Topography and Social Conflict, Baltimore 1997.

Hahn, J., Tempelzerstörung und Tempelreinigung, in: Albertz, R. (Hrsg.), Kult, Konflikt, Sühne (Veröffentlichungen des Arbeitskreises zur Erforschung der Religions- und Kulturgeschichte des Antiken Vorderen Orients, Band 2), Münster 2000, 269-285.

Hahn, J., Gewalt und religiöser Konflikt. Studien zu den Auseinandersetzungen zwischen Christen, Heiden und Juden im Osten des römischen Reiches (von Konstantin bis Theodosius II.) (Klio-Beihefte N.F. 8), Berlin 2004.

Hahn, J., Vetus error extinctus est - Wann wurde das Sarapeion von Alexandria zerstört?, Historia 55 (2006), 368-383. 
Hahn, J., The Conversion of the Cult Statues: The Destruction of the Serapeion 392 A.D. and the Transformation of Alexandria into the „Christ-Loving City“, in: Hahn, J. - Emmel, St. - Gotter, U. (Hrsg.), From Temple to Church: Destruction and Renewal of Local Cultic Topography in Late Antiquity (Religions in the Graeco-Roman World 163), Leiden 2008, 335-365.

Harries, J., The Background to the Code, in: Harries, J. - Wood, I. (Hrsg.), The Theodosian Code. Studies in the Imperial Law of Late Antiquity, Ithaca 1993, $1-16$.

Harries, J., Law and Empire in Late Antiquity, Cambridge 1999.

Hermanowicz, E.T., Catholic Bishops and Appeals to the Imperial Court: A Legal Study of the Calama Riots in 408, JECS 12 (2004), 481-522

Hermanowicz, E.T., Possidius of Calama. A Study of the North African Episcopate, Oxford 2008.

Honoré, T., Law in the Crisis of Empire 379-455 A.D. The Theodosian Dynasty and its Quaestors, Oxford 1998.

Horn, J., Studien zu den Märtyrern des nördlichen Oberägypten I: Märtyrerverehrung und Märtyrerlegende im Werk des Schenute (Göttinger Orientforschungen, IV. Reihe: Ägypten, Bd. 15), Wiesbaden 1986.

Humfress, C., Orthodoxy and the Courts in Late Antiquity, Oxford 2007.

Hunt, E.D., Christianizing the Roman Empire. The Evidence of the Code, in: Harries, J. - Wood, I. (Hrsg.), The Theodosian Code, Ithaca 1993, 143-158

Isele, B., Kampf um Kirchen. Religiöse Gewalt, heiliger Raum und christliche Topographie in Alexandria und Konstantinopel (4. Jh.). JbAC Erg.bd., kl. Reihe 4, Münster 2010.

Joannou, P., La législation impériale et la christianisation de l'empire romain, 311-476 (Orientalia Christiana Analecta 192), Rome 1972.

Lenski, N., Evidence for the audientia episcopalis in the New Letters of Augustin, in: R.W. Mathisen (Hrsg.), Law, Society, and Authority in Late Antiquity, Oxford 2001, 83-97.

Lenski, N., Failure of Empire. Valens and the Roman State in the Fourth Century A.D., Berkeley 2002.

Leppin, H., Constantius II. und das Heidentum, Athenaeum 87 (1999), 457-480.

MacMullen, R., Christianity and Paganism in the Fourth to Eighth Centuries, New Haven - London 1997.

Marique, J.-F., A Spanish Favorite of Theodosius the Great: Cynegius, Praefectus Praetorio, Classical Folia 17 (1963), 43-65.

Matthews, J. F., Laying Down the Law. A Study of the Theodosian Code, New Haven 2000.

McLynn, N., 'Genere Hispanus': Theodosius, Spain and Nicene Orthodoxy, in: K. Bowes - M. Kulikowski (eds.), Hispania in Late Antiquity: Current Perspectives, Leiden 2005, 77-120.

Meier, H.-R., Alte Tempel - neue Kulte. Zum Schutz obsoleter Sakralbauten in der Spätantike und zur Adaption alter Bauten an den christlichen Kult, in: B. 
Brenk (ed.), Innovation in der Spätantike. Kolloquium Basel 1994, Wiesbaden 1996, 363-376.

Millar, F., Pagan and Christian Voices from Late Antiquity, JRA 13 (2000), 752762.

Millar, F., A Greek Roman Empire. Power and Belief under Theodosius II. (408450) (Sather Classical Lectures 64), Berkeley 2006.

Noethlichs, K.-L., Die gesetzgeberischen Maßnahmen der christlichen Kaiser des 4. Jahrhunderts gegen Häretiker, Heiden und Juden, Diss. Köln 1971.

Noethlichs, K.-L., Beamtentum und Dienstvergehen. Zur Staatsverwaltung in der Spätantike, Wiesbaden 1981.

Lizzi Testa, R., Senatori, popolo, papi. Il governo di Roma al tempo dei Valentiniani, Bari 2004.

Noethlichs, K.L., s.v. Heidenverfolgung, RAC 14 (1986), 1149-1196.

Paschoud, F. (Hrsg.), Zosime, Histoire Nouvelle II 2: Livre IV, Paris 1979.

Ombretta Cuneo, P. (Hrsg.), La legislazione di Costantino II, Costanzo II e Costante (337-361), Milano 1997.

Pergami, F., La legislazione di Valentiniano e Valente (364-375), Milano 1993.

Rosen, K., Iudex und officium: Kollektivstrafe, Kontrolle und Effizienz in der spätantiken Provinzialverwaltung, AncSoc 21 (1990), 273-291.

Rougé, J., Valentinien et la religion 364-365. Ktéma 12 (1987), 285-297.

Salzman, M.R., 'Superstitio' in the Codex Theodosianus and the Persecution of Pagans, VChr 41 (1987), 172-188.

de Ste. Croix, G.E.M., Christian Persecution, Martyrdom, and Orthodoxy. Oxford 2006.

Testa, E., Legislazione contro il paganesimo e cristianizzazione dei templi (sec. IV-VI), Liber Annus 41 (1991), 311-326.

Trombley, F., Hellenic Religion and Christianization c. 370-529, Bde. I-II, (Religions in the Graeco-Roman World 115), Leiden 1993/1994.

Vessey, M., The Origins of the Collectio Sirmondiana. A New Look at the Evidence, in: J. Harries - I. Wood (eds.), The Theodosian Code, Ithaca/N.Y. 1993, 178-199.

Wessely, C. (Hrsg.), Griechische und koptische Texte theologischen Inhalts I, (Studien zur Palaeographie und Papyruskunde 9), Leipzig 1909. 


\section{Register}

\section{Personen-, Orts- und Sachregister}

Abgar 179

Admedera 34

Administration, römische $\quad 135,139$ 140

Africa 81, 93-97, 120

Agrippa II. 142-143

Ägypten 190-191

Akoris, Ägypten 35

Albinus, Rufius 78

Alexandria 58, 71, 77, 215

Alypius 211

Ambrosius 75, 77, 133-134, 145147

Ambrosius von Mailand 48

Ammaedara 38

Ammianus Marcellinus 25, 32, 36, 70

Anonymus de rebus bellicis $\quad 62-63$

Antinoopolis, Ägypten 37

Antiochia, Syrien 36, 45, 58, 171174, 176-177

Antiochos IV. 144

Antoninus von Fussala 211

Apamea 173

Aphrodisias 192, 195

Aphrodite-Kult 13

Apollodorus, procos. Africae 80

Aquileia 134-135

Arabia 33

ARCADIUS 80, 107, 172

Archäologie 187-196

Asklepiodotos, PPO 83

Asterius, comes Orientis 79

Aufruhr 107, 120-121, 124
Augustinus $211,49,95,99,121$, 125

AURELIAN 21, 22, 46

Babylas 175-176

Baiae, Vespasian-Tempel 35

Baitokaikai, Phoenikien 34

Beamte, kaiserliche 209-215

Beroia 166

Bischof, Bischöfe $\quad 70,81,94-96,99$, 104,109, 117-120, 122-123, 128, $145,149,162,175,211-215$

Breno, Minerva-Tempel 193

Brixia 21

Cabassa, Narbonensis 28

Caesarea, Mauretania 26

Caesarius, PPO 79

Calama 213

Capua 36

CARINUS 21-22

CARUS 21-22

Chester, Anglia 26

Christianisierung 203, 97-98, 116117, 160-161, 165, 174, 178

Chronicon Paschale 64

Cingulum, Picenum 21

Cirta, Numidia 25

Claudius Gothicus 21, 29

Codex Theodosianus 75, 96, 98,

$111,117,170,187,201-210$

Comes orientis 133,146

Constantius I. 22-23, 26-27

COnstantius II. 27-28, 67-69, 71, 109, 176-177, 205-206

CRISPUS 165 
Cynegius Maternus, PPO 77, 122, $160,163,166-171,178,213-214$

Cyprian von Carthago 41

Damasus I., Papst 75

damnatio memoriae 20-24, 31, 34, 36,45

Daphne 122, 126

Daphne, Apollon-Tempel 175, 177178

DECIUS 21

Dendera 191

Desakralisierung 207-209

DIOKLETIAN 21-24, 44

Donatisten 93-95

Dura Europos 188

Durostorum, Moesia Inf. 30, 44

Dyrrachium 23

St. Dasius 30, 41, 44

Edessa 73

Edessa, Osrhoene 166, 178-180

Cunctos populos, Edikt 205

Egeria 179

Eigentumsübertragung $55,59,62$, $64,71,80$

Eleusis 51

Emesa 173

Entsakralisierung $\quad 59,69,71,177$

Ephesus 35, 51, 190

- Hadrian-Tempel 35

Eretria, Euboea 31, 50

Eugenius 78-7

Eunapios 102

Eusebius von Caesarea 7-18, 19, 23, $26,29,41-43,45,47,55-58,60-$ $63,100,179,189$

- Tricennalienrede 55, 58, 65

- Vita Constantini 55,60-61

FAUSTA 165

Festwesen 112-113

Firmicius Maternus 61-63

Flavianus, Bischof

von Antiochia 175-176

Flavius Josephus 142-143

FLORIANUS 20

Frigidus, Schlacht am 74, 79
GALERIUS 23, 24, 26-27, 29, 31

GALLIENUS 21, 34

GALLUS 21

Gaza 173, 212

generalitas von Gesetzen 205-206

Gerasa 33

Gesetzgebung, Eigenart

- spätantike 209-216

- anti-häretische 90,152

- anti-pagane 9-10, 15, 76-82, 93-94, 99, 103, 108-109, 111, 152, 192, 201-209, 213-216

Gewalt 162-165, 167-168, 171$172,174-175,178$

Gewalt gegen Kultorte 133-136

Gewalt(anwendung), religiös motiviert 133-153

Gewaltdiskurs 145-148

Gigithi, Byzancena 25

Grabeskirche 66

Gracchus, Furius

Maecius, PV 75

GRATIAN 24-25, 75-76, 82

Gregor von Nazianz 70

Häretikergesetze 99,152

Harpinium 28

,Heidentum“ (Begriff) 9

Heliopolis-Baalbek 13, 173

Herrscherkult 19-25, 29-39, 44-46, $48-50,60-61,83$

Hieronymus 48, 65

Hilarius, Bischof

von Poitiers $62,65,71$

Hispellum 29-30, 37, 60

Historia acephala 71

Historiographie, christliche 117, 206

HONORIUS $67,75,79,82,93,95$, $100,107,113,116,120$

Hypatia 83

Jerusalem, Grabeskirche 12-13

Johannes Chrysostomos 175-176, 187

JOVIAN $24,36,72,177$

Judäa 141-142

1. Jüdischer Aufstand 141-144 
jüdische Elite 141-143

JULIAN 24, 32, 71-73, 100, 104, 111, 118, 161, 165-166, 175, 177-179, 204

JUSTINIAN 19,187

Kaiserkult 15-16, 160

Kaisertum 159-160, 169

Kaisertum, christliches 146-152

Kallinikon 133-136, 145-147

Karrhai 173,179

Karthago 81, 93-94

Kirche, nordafrikanische 211-214

Kirchenbau 66, 74

Konfiskation 160-161, 165, 207

Konflikt, religiöser 93-97, 124, 133-134, 145, 148-153

KONSTANTIN 7-18, 24, 26-29, 32, 45, 47-48, 55-67, 100, 111, 125, 148, 159-160, 165, 170, 176, 188-189, 205

- Religiosität 16-17

Konstantinopel 14, 58, 65, 66-67

Konversion 165

Koptos, Ägypten 32

Kultgegenstände 82

Kultstatuen 58

Kultstatuen (pagane) 9, 14-15

Kyrene 126, 193

Kyrill von Alexandria 211

Laktanz 23, 29, 32, 36

Lambaesis, Numidia 22

Lampadius, C. Ceionius Rufius Volusianus 74

Laterankirche, Rom 66

LEO I. 109

Leon, Gallaecia 42

Libanios 58, 124-127

Libanios, Epitaphios 71-72

Libanios, Pro Templis $\quad 69,76,111$, 162-180

Luna 189

Madalianus, L.Crepereius 65

Makarios von Jerusalem 66

Makkabäer 143-144

makkabäische Brüder 133
Mamertinus, PPO 73

Mamre 10-12

Manichäer 99

Marcellus von Apameia 109, 213

Marcellus, Märtyrer 42-43

M. Iulius Philippus 21

Marinus, Märtyrer 41

Markos von Arethusa 70-71

Marnas-Tempel, Gaza 107, 121, 193-194

Martin von Tours 187

Märtyrerakten 40-42

Maßnahmen, anti-pagane 7-18

MAXENTIUS 23-24, 29

MAXIMIANUS 22-25, 28,36

MAXIMINUS DAIA 26, 29

Medinet Habu 191

Meletius, Bischof 176

Meron, Phrygia Salutaris 34

Mesopotamia 32

Moesia Inferior 22

Mönche 70, 133-134, 163-164, $168,172,174-176,209$

Münzprägung 62-64

Nicomachus Flavianus,

Virius 74,78

Nicomedia 32

Nikopolis, Moesia inf. 35

Nizäner 94-95, 97, 99

Normen, imperiale 135, 139

notarii 213-214

NUMERIANUS 21-22

officiales 100, 103- 104, 113-115, $120,122-123,125,210$

Opfer, Opferverbot $\quad 9-12,15-16,56$, $60,67,76-77,161,164,166-$ 167, 206-207

Oriens, Diözese 172

Origines 19

Origines, Contra Celsum 45-46

Palladius, dux Osrhoenae 73

Panopolis 201

parabalani 211

Passio Iuli veterani 43

Pax Romana 135 
St. Peter, Rom $\quad 66,188$

Philae 187

Philippopolis 33

PHILIPPUS ARABS 19-20, 21, 33, 46

Philostorgius 37,48

Pionius, Märtyrer 41

Plutarch 140

Porphyrios von Gaza 93-94, 104, $107,109,114,119,121,126$

Porphyrius von Tyros 30

Praetextatus, Vettius Agorius 74, 100,210

Priester 38-39, 49

privilegium 101, 108, 119

Probus 20

Proclianus, PPO 79

QUIETUS, Usurpator 32-33

Ratsherren $172,176-178$

Ravenna 211

Reichsverwaltung 209-212

Religionsgesetzgebung 93-97, 103109, 115-116, 124-125, 161, $170,173,201-216$

Religionspolitik, kaiserliche 59-62, 69-73,75, 79-84, 97-101, 111$112,202-208,215-216$

res privata $59,62,64-66,71-72$, $75,76,80,82-83$

Restauration, heidnische $71-72,74$, $78,161,178$

Rom 58, 65, 66, 69, 73, 74, 77, 165, 189,194

ROMULUS 27

Rufinus von Aquileia 49, 215

Saint-Aubin-sur-Mer 194-195

SALONINUS, P. Licinius 21

Santa Prisca, Rom (Mithräum) 194

Sarsina 195

Schenute von Atripe $103,124,187$, 201-202, 216

Schutz heidnischer Kulte 73-74

Schutz von Tempeln 112

Serapeion, Alexandria 77, 111, 215

Sibyllinische Bücher 82

Sirmium 27
Smyrna, Kaisareion 35

Sokrates, Kirchenhistoriker 61

Sozomenos, Kirchenhistoriker 71, $116,206,213-215$

städtische Elite 159-162, 173

stasis 151

Statthalter 93, 96-104, 107-108, 111-114, 117-118, 120-

$128, \quad 208,210-213$

Statuen $35-37,48,188-191$

Stilicho 82, 95

Strafmaß 106-107, 109-110, 124

Strafprozesse 108, 112-113

suggestiones 211

Symmachus, Q. Aurelius 73, 74, 75

Synagoge(n), Zerstörung 133-136

Synesios 125-126, 128

Syria Coele, Provinz 173, 175

Tatianus, Fl. Eutolmius, PPO 37, 163

Tempel 30-38, 44, 55-61, 164-165, 188-192, 205

- Bewahrung 69-70, 73, 77, 79$81,166,207$

- Plünderung 70-71, 77, 79, 160, 172

- Schließung 55, 67, 71, 75, 160, 188, 191-192, 207-208, 213

- Umnutzung 62, 68, 70,81, 177, 191, 193, 195-196, 207

- Verlassen 189, 191-192, 195196

- Zerstörung 7, 9, 56-57,6870,71-72, 75, 77, 79-84, 160, 163-164, 166-168, 171-172, 174, 178-179, 187, 193-195, 207-209, 212-215

Tempelgut $55,58-59,63-64,70$ $72,78-80$

Tempelprostitution 13-14

Tetrarchie 22-24

Thamugadi, Numidia 21, 38

Theodoret 174-175, 213 
Theodosius I. 19, 25, 49, 75-76, 79, 101, 108-111, 116, 133-136, 145-148, 161-162, 163-165, 166-167, 169-171, 172-173, 176-178, 192, 205-207

TheOdosius II. $\quad 82-83,102,201-$ 202

Theophilos, Bischof

von Alexandria 77, 215

Thessaloniki 24

Thuburuscum, Numidia 21

Tiberius Alexander 141

Tieropfer s. Opfer

Tingis, Mauretania 42

Trajansforum 28

Trebonianus Gallus 21

Troesmis, Scythia Minor 28
Unterdrückung paganer Kulte 9798, 100-101, 105-107, 110, 112, $118,121-122$

VALENS 25, 72-73,161, 210 170, 176

VALENTINIAN I $24-25,72-73,170$, 204, 210

VALENTINIAN II. $\quad 75,83$

VALERIAN 21, 34

Vegetius 30

Verecundia 22

Vergöttlichung 21-30,33, 40

Verona, Capitolium 192

Vesta-Kult 75-76

Vicetia 28

Victoria-Altar 69,75

Vita Porphyrii 212-213

Zosimos 10

\section{Quellenregister}

Literarische Quellen

Acta Agap. 3105

Acta conc. oec. IV 1: 155104

Acta Marcelli, rec. M 142

- N 343

Acta procos. S. Cypr. 142

Afric. chronogr., frg. 1810

Ambr. ep. 74 (40) 133-136, 145148

Ambr. ep. extr. coll. 1 133-134

Ambr. hexaem. 6,9,57 49

Amm. 23,5,7-8 32

- 25,10,1-2 36

- 30,7,2 25

Anon. de rebus bell. 2,1f. 63

- 2,4 63

Aug. civ. XVIII 54120

Aug. ep. 91,8 94

- 232,1 98

Chron. Pasch. ad a. 32564

Epikt. diss. 4,1,14 45

Eun. F 29 Blockley 177
Eun. v. soph. $503 \quad 102$

Eus. d. e. 5,9,7-8 12

Eus. h. e. 8,1323

Eus. 1. C. $7,13 \quad 55$

- 8,2-3 58

- 8,313

- 8,5-7 13-14

Eus. mart. Pal. 3,1 44

Eus. v. C. 1,21 26

- $2,45,19$

- 2,46,3 66

- 2,48-60 61

- $3,1,5 \quad 57$

- 3,26,2 13

- $3,52 \mathrm{f} .12$

- 3,53,2 9

- $3,54,1$ f. 7

- 3,54189

- $3,54,214$

- $3,54,314$

- 3,56-58 57 
- 3,58,3 13

- $4,1615,61$

- 4,1947

- 4,25 14

- 4,25,1 56

- 4,39,2 7

- 4,60 16

Firm. err. prof. rel. 28,5 62

- 28,6 62

Hieron. comm. in Ier. VI 18,6 11

Hieron. chron. ad a. 33165

Hieron., exeg. in Dan. 3,18 48

Hilar. ep. ad Const. 1, $6 \quad 62$

Itin. Egeriae 19,2-17 180

Jo. Chrys. de S. Babyla 67-113 175

Jo. Chrys. hom. 1-8 adv. Iud. 176

Jo. Chrys. hom. 4,6 in 1. Cor. (PG 61, 38-40) 176

Jo. Chrys. hom. 15,5 in Phil. (PG 62, 295) 165

Jo. Mal. chron. 13,13 14

Joseph. bell. 2,361-365 142-143

Lact. mort. pers. 19,2 32

- 4223

Lib. ep. 958171

Lib. or. 12,69 169

- 12,79-82 169

- 18,23111

- 18 (Epit.), 126 71-72

- 30 (Pro Templis) 162-180

- 30,7 161

- $\quad 30,10169$

- 30,3710

- 30,52-53 160-170

- 50,36169

- 62,859
1Makk. 3,16-23 144

Mart. Das. 731

- 7-8 44

Mart. Polyc. 839

M. Diac. v. Porph. 69193

- 75-79 193

Orig. c. Cels. 8,65 45

- 8,6646

- 8,6846

- 8,7346

Paneg. X (II) 10,6,5 37

- $\quad$ XI (III) 2,4 23, 26

Passio S. Marini = Eus. h. e. 7,15 41

Passio S. Pionii $8 \quad 41$

Passio SS. Scillit. $3 \quad 40$

- 6,9 40

Philost. h. e. $2,17 \quad 15$

Plin. ep. 10,34,1 138-139

Plut. mor. 813d-f 140

Porph. De abstin. 2,35,40-43 30

Prok. bell. Pers. 2,12,26 179

- $1,19,37187$

Quodvultd. I prom. 13,15 125

Schenoudi, Oeuvres (ed. É. Amélineau), t. II, fasc. 3, 1914, p. 523,7201

Socr. h. e. $1,18,1 \quad 61$

Soz. h. e. $2,4,3 \quad 11$

- 7,12,12 116

Thdt. h. e. 4,24,2 111

- 5,21,5 213

Veget. mil. 2,5 30

Zos. 2,31 14

- 4,3,2-3 161, 210

- 4,3,3 102

Gesetzestexte

Cod. Iust. 4,35,23-24 50

Cod. Theod. 1,1,5 pr. 204

- 9,16,7 161

- 9,16,9 204

- $15,1,3679,173$

- $16,1,173$
- $\quad 16,10,2 \quad 10,205-206$

- $16,10,3 \quad 68,207$

- 16,10,4 67

- $16,10,8 \quad 166,169$

- $16,10,1278$

- $16,10,15 \quad 80,81,207$ 
- $\quad 16,10,16 \quad 80,216$

- $16,10,17217$

- $16,10,1981,82$

- $16,10,20 \quad 82$

- $16,10,21 \quad 102$

- $16,10,2483$

$$
\text { - } \quad 16,10,25 \quad 84,203
$$

Const. Sirmond. 12 93, 94, 109, $113,117,120,123,213$

- 14 95, 96, 103, 107, 114, 115, 120,213

Nov. Theod. 3,8 103, 110

\section{Inschriften}

CIL 3,7586 21

CIL 5,3332 192

CIL 8,2387 38

CIL 8,2403 38

CIL 8,7034 38

CIL 8,10516 38

CIL 8,17896 38

CIL $115265=$ ILS $705 \quad 15,101$

IG *II 2,5206 39

IGR 1,1181 33

IGR 1-2,582 36

IGR 1-2,1134 35

IGR $3,873 \quad 35$

IGR 3,1020= CIL 3,184 35

IGR 3,1093 = Waddington $2562 \mathrm{~g} \quad 34$

IGR 3,1094 34

IGR 3,1199= Waddington 207533

IGR 3,1200a 33

IGR 3,1286 35

IGR 3,1363 33

IGR 4,592, $593 \quad 34$

IGR 4,1480 35

ILS $540 \quad 34$

ILS $556=$ CIL 9,5682 21

ILS $557=$ CIL 8,8473 21

ILS 55821

\begin{tabular}{|c|c|c|}
\hline ILS 559 & & \\
\hline ILS 579 & 21 & \\
\hline ILS 585 & 22 & \\
\hline ILS $593=$ & $=$ CIL 2,1115f. & 21 \\
\hline ILS 608 & 22 & \\
\hline ILS $609=$ & $=$ CIL 8,4221 & 22 \\
\hline ILS $618=$ & $=\mathrm{CIL} 3,5810$ & 45 \\
\hline ILS $621=$ & $=$ CIL 6,254 2 & 22 \\
\hline ILS $622=$ & $=$ CIL 6,255 & 22 \\
\hline ILS $629=$ & $=\mathrm{CIL} 3,710$ & 23,47 \\
\hline ILS 634 & 24 & \\
\hline ILS 671 & 27 & \\
\hline ILS $673=$ & $=$ CIL 6,1138 & 27 \\
\hline ILS 682 & 26 & \\
\hline ILS $692=$ & $=$ CIL 6,1140 & 28 \\
\hline ILS 693 & 28 & \\
\hline ILS $697=$ & $=$ CIL 5,8011 & 28 \\
\hline ILS $699=$ & $=$ CIL 11,9 29 & \\
\hline ILS 702 & 29 & \\
\hline ILS $732=$ & $=$ CIL 3,3705 & 28 \\
\hline ILS $758=$ & $=$ CIL 8,7014 & 25 \\
\hline ILS $779=$ & $=$ CIL 8,10489 & 25 \\
\hline ILS 4175 & 35 & \\
\hline ILS 4918 & $=$ CIL 10,3792 & 37 \\
\hline ILS 8881 & 42 & \\
\hline OGIS 723 & & \\
\hline
\end{tabular}

ILS $559 \quad 21$

ILS 58522

ILS $593=$ CIL 2,1115f. 21

22

ILS $618=$ CIL 3,5810 45

ILS $621=$ CIL 6,254 22

ILS $622=$ CIL 6,255 22

ILS $629=$ CIL 3,710 23, 47

ILS 63424

ILS $673=$ CIL 6,1138 27

ILS 68226

ILS $692=$ CIL 6,1140 28

ILS 69328

ILS $697=$ CIL 5,8011 28

ILS $699=$ CIL 11,9 29

ILS 70229

ILS $732=$ CIL 3,3705 28

ILS $758=$ CIL 8,7014 25

ILS $779=$ CIL 8,10489 25

ILS $4175-35$

ILS $8881 \quad 42$

OGIS 72337 


